\title{
SOBIR1-containing immune complexes at the plant cell surface: partners and signalling
}

Aranka M. van der Burgh 


\section{Propositions}

1. Immune signalling by receptor-like proteins (RLPs) requires transphosphorylation of SOBIR1 and BAK1.

(this thesis)

2. Mechanisms of plant immunity should be described based on extracellular versus intracellular recognition of invasion patterns that are sensed upon attack by invading organisms.

(this thesis)

3. Organic agriculture and modern breeding techniques, such as genetic engineering, must go hand in hand to improve agriculture in a sustainable manner.

4. Our tendency to describe biology by using models hampers our mind to truly understand nature.

5. Choosing appropriate controls is the most important aspect of a good experimental design.

6. Competition is a disease that scientists need to deal with.

7. Meat consumption must be reduced from an everyday routine to something special once a week.

Propositions belonging to the thesis, entitled

'SOBIR1-containing immune complexes at the plant cell surface: partners and signalling.'

Aranka Margrethe van der Burgh

Wageningen, 21 September 2018 


\section{SOBIR1-containing immune complexes at the plant cell surface: partners and signalling}

Aranka M. van der Burgh 


\section{Thesis committee}

\section{Promotor}

Prof. Dr Bart P.H.J. Thomma

Professor of Phytopathology

Wageningen University \& Research

\section{Co-promotor}

Dr Matthieu H.A.J. Joosten

Associate professor, Laboratory of Phytopathology

Wageningen University \& Research

\section{Other members}

Prof. Dr Dolf Weijers, Wageningen University \& Research

Prof. Dr Guido F.J.M van den Ackerveken, Utrecht University

Dr Aska Goverse, Wageningen University \& Research

Dr Andrea A. Gust, University of Tübingen, Germany

This research was conducted under the auspices of the Graduate School Experimental Plant Sciences (EPS). 


\section{SOBIR1-containing immune complexes at the plant cell surface: partners and signalling}

Aranka Margrethe van der Burgh

Thesis

submitted in fulfilment of the requirements for the degree of doctor at Wageningen University

by the authority of the Rector Magnificus,

Prof. Dr A. P. J. Mol,

in the presence of the

Thesis Committee appointed by the Academic Board to be defended in public on Friday September 21, 2018

at 4 p.m. in the Aula. 
Aranka M. van der Burgh

SOBIR1-containing immune complexes at the plant cell surface: partners and signalling, 248 pages.

PhD thesis, Wageningen University, Wageningen, The Netherlands (2018)

With references, with summaries in English and Dutch

ISBN: 978-94-6343-313-6

DOI: $10.18174 / 454717$ 


\section{Table of Contents}

Chapter 1 General introduction

Chapter 2 The bacterial effector AvrPto targets the regulatory co-receptor SOBIR1 and suppresses defence signalling mediated by the receptor-like protein $\mathrm{Cf}-4$

Chapter 3 Transphosphorylation between SOBIR1 and BAK1 is required for immune signalling

Chapter 4 Phosphorylation of specific residues of the kinase domain of SOBIR1 is essential for its role in immune signalling

Chapter 5 RLCKs signal for immunity downstream of SOBIR1 in Solanaceous plants

Chapter 6 Studies on BIR1 and its possible role as a negative regulator of Cf-4/SOBIR1-mediated immunity in Solanaceous plants

Summary

Samenvatting

Acknowledgements

About the author

List of publications

List of abbreviations 



\title{
Chapter 1
}

\author{
General introduction
}


Plant cells communicate with each other and with the environment, but how does this communication work? In the processes leading to plant resistance against pathogens, communication is essential. Plants do not have an adaptive immune system like mammals. Nevertheless, the mechanisms by which plants combat diseases are versatile and intriguing. If we want to feed the ever growing human population on earth, we must improve our agricultural practices. We can learn from nature by deciphering the molecular mechanisms behind plant resistance. The acquired knowledge on the immune system of plants will help to minimize crop losses in a sustainable way. Implementing natural resistance mechanisms in susceptible crop plants will be an important step to secure food production in a sustainable fashion in the future.

\section{The 'outs and ins' of the plant immune system}

Plants are sessile organisms with multiple layers of defence against pathogens (Dodds \& Rathjen, 2010). First layers of defence are non-inducible phenomena, for example a waxy film on the leaves, a protective microbiome on the plant surface to prevent pathogens from entering, and the presence of antimicrobial compounds (phytoanticipins) like $\alpha$-tomatine (Martin, 1964; Hückelhoven, 2008; Sandrock \& van Etten, 1998). The next layer of defence, which is similar to the innate immunity of mammalian cells (Akari et al., 2006), is provided by transmembrane (TM)-receptors, which are present at the cell surface (Fig. 1A). TM-receptors have an extracellular domain (ECD) to detect extracellular signals (Zipfel, 2014). Upon pathogen invasion of the extracellular (apoplastic) space, TM-receptors mediate recognition of invasion patterns (IPs; see BOX 1). IPs in the extracellular space can be damage-associated molecular patterns (DAMPs), which are plant components that are released upon pathogen invasion, microbe-associated molecular patterns (MAMPs) produced by the invading microbe (also called pathogen-associated molecular patterns, PAMPs), or effectors produced by the invader that accumulate in the apoplast. MAMPs are structural components of microbes, such as chitin and lipo-polysaccharides (Jones \& Dangl, 2006; Dodds \& Rathjen, 2010; Zipfel, 2014; Macho \& Zipfel, 2014; Böhm et al., 2014a; Cook et al., 2015). Microbes that enter the cytoplasm of the host cells, or that secrete, translocate, or inject their effectors into the host cells, in their turn can be detected by intracellular immune receptors (Fig. 1A) (Dodds \& Rathjen, 2010; Win et al., 2012). Both extracellular and intracellular recognition eventually signal for the activation of defence responses, leading to resistance. 


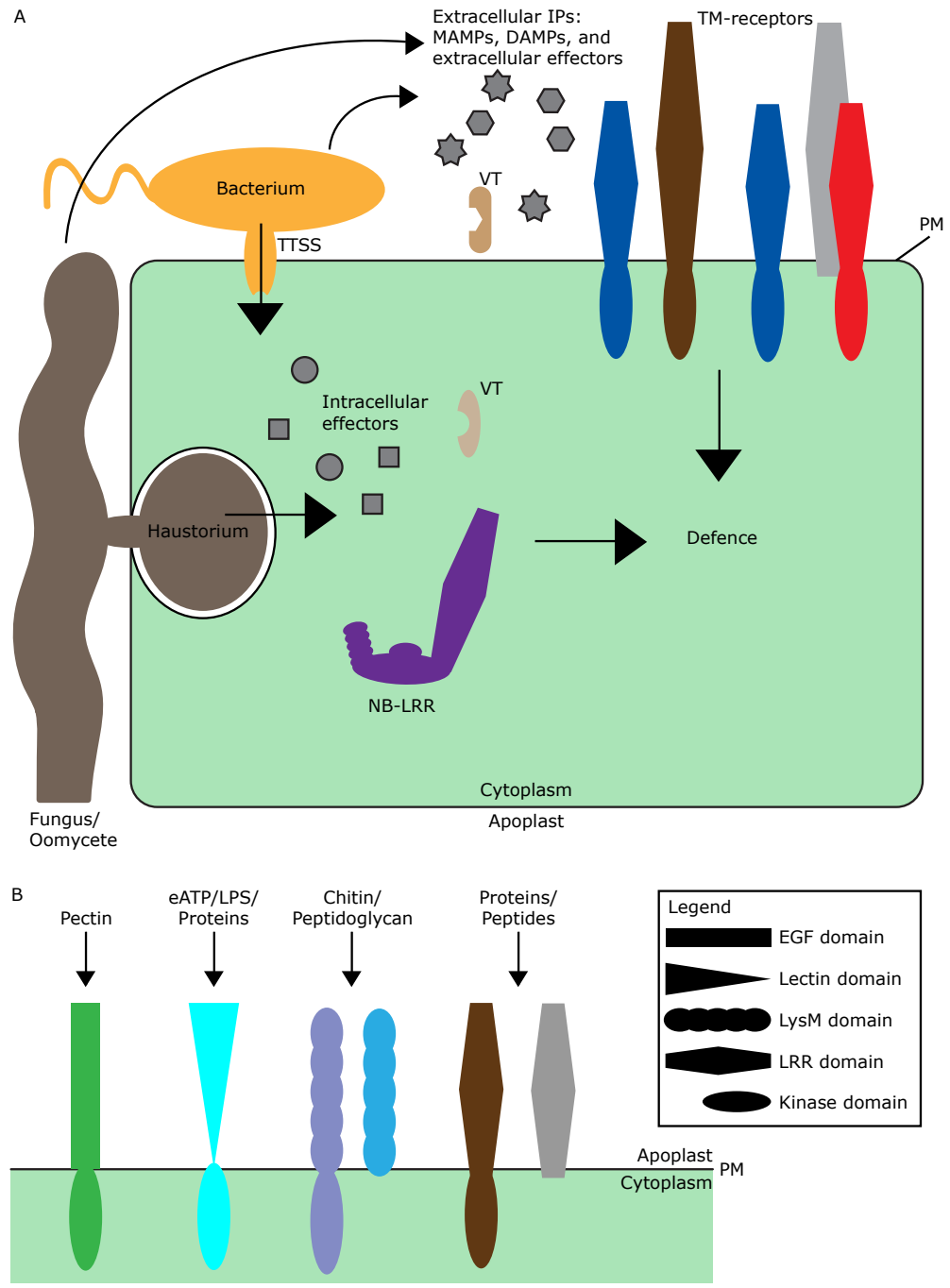

Figure 1. Schematic overview of the plant cell with receptors able to trigger defence responses, localised at the cell surface and in the cytoplasm. (A) Extracellular invasion patterns (IPs) of microbes like bacteria, fungi, and oomycetes, or the effect that they have on apoplastic host virulence targets, can be sensed via transmembrane (TM)-receptors that are present on the PM. These extracellular IPs can be MAMPs and effectors, originating from the invading microbe, or DAMPs originating from an action of the pathogen to the plant. Intracellularly secreted effectors, or their effect on cytoplasmic host virulence targets, can be sensed by cytoplasmic NB-LRR receptors. Both extracellular and intracellular recognition leads to the activation of defence responses. Picture inspired by Dodds \& Rathjen (2010). (B) TM-receptors can perceive different extracellular signals via various extracellular domains (ECDs). PM, plasma membrane; MAMP, microbe-associated molecular pattern; DAMP, damage-associated molecular pattern; TTSS, type three secretion system; NB-LRR, nucleotide binding leucine-rich repeat; VT, virulence target; LPS, lipo-polysaccharides; EGF, Epidermal Growth Factor; LysM, lysin motif; LRR, leucine-rich repeat. 


\section{Sensing 'the outs'}

TM-receptors can be subdivided into receptor-like kinases (RLKs) and receptor-like proteins (RLPs), of which the latter lack an intracellular signalling domain (Fig. 1B). The ECD is responsible for recognition of extracellular signals. Different kinds of ECDs can recognize different signals (Böhm et al., 2014a; Zipfel \& Oldroyd, 2017). Typical ECDs of TM-receptors are: the Epidermal Growth Factor (EGF) domain, the lectin (Lec) domain, the lysin motif (LysM) domain, and the leucine-rich repeat (LRR) domain. EGF-like receptors recognize pectin fragments of different lengths (Kohorn et al., 2012). Pectin originates from the plant cell wall, and EGF-like receptors interact with pectin that resides in the cell wall to ensure cell expansion. Additionally, released pectin fragments, oligogalacturonic acids (OGs), can act as DAMPs to signal for defence and stress responses (Brutus et al., 2010; Kohorn et al., 2012). Lectin receptor kinases (LecRKs) have been associated with the recognition of several molecules like extracellular ATP (eATP), various lipopolysaccharides, and proteins (Choi et al., 2014; Ranf et al., 2015; Singh \& Zimmerli, 2013; Wang \& Bouwmeester, 2017). LysMRLKs recognize fragments of the fungal cell-wall component chitin or bacterial peptidoglycan (Gust et al., 2012; Gust et al., 2017; Zipfel \& Oldroyd, 2017). Last but not least, LRR-RLKs and LRR-RLPs are known to recognize a range of ligands, from Brassinosteroid hormones to a plethora of peptides and proteins (Li \& Tax, 2013; Wang et al., 2010). Recognition can take place either directly or indirectly via the recognition of the effect that pathogens have on so-called virulence targets (Kourelis \& van der Hoorn, 2018).

By recognition of pathogen attack in an indirect way, the manipulation of a host virulence target (also called guardee) by an invading pathogen is detected, which triggers defence signalling. Recognition of the manipulation of a guardee by an effector is considered to be an evolutionary more durable way of recognition (BOX 1), as in this way not the highly variable effector itself is being recognised, but the action of the effector that benefits the pathogen (van der Hoorn et al., 2002). Pathogen recognition following this guard mechanism can only be circumvented by avoiding the manipulation of the guardee, which results in a fitness penalty for the pathogen (van der Hoorn et al., 2002).

Defence signalling upon recognition of extracellular proteins and peptides by LRR-RLKs and LRR-RLPs (further referred to as RLKs and RLPs) is a well-studied subject. A famous example of an RLK involved in immunity to bacteria is FlagellinSensing 2 (FLS2). FLS2 recognizes the bacterial peptide flg22, a typical MAMP which is derived from the bacterial flagellum (Gómez-Gómez \& Boller, 2000). Upon recognition of flg22, FLS2 associates with the co-receptor Brassinosteroid-Insensitive 1 (BRI1)-Associated Kinase 1 (BAK1), also known as Somatic Embryogenesis Receptor Kinase 3 (SERK3), further referred to as BAK1 (Fig. 2A) (Chinchilla et al., 2007; Heese 
et al., 2007). The LRRs of FLS2 and the co-receptor BAK1 have both been shown to physically interact with flg22 (Sun et al., 2013). The recruitment of BAK1 appears to be a central event in signalling by TM-receptors, and it initiates a signalling cascade leading to the activation of several defence responses (Chinchilla et al., 2009; Schulze et al., 2010; Ma et al., 2016; Hohmann et al., 2017; Hohmann et al., 2018).

\section{RLPs constitutively interact with SOBIR1 and recruit BAK1 upon their activation}

RLPs, which as mentioned earlier do not contain a cytoplasmic signalling domain, constitutively interact with the RLK Suppressor Of BAK1-Interacting RLK-1 (BIR1)-1/ Evershed (SOBIR1/EVR, further referred to as SOBIR1) (Liebrand et al., 2013). This constitutive interaction is proposed to provide a kinase domain to RLPs, and together they form a kind of bimolecular RLK (Liebrand et al., 2014; Gust \& Felix, 2014). SOBIR1 was initially found to interact with the $\mathrm{Cf}$ proteins from tomato (Solanum lycopersicum, Sl; conferring Cladosporium fulvum (Cf) resistance), with tomato Ve1 (conferring Verticillium dahliae resistance), tomato Ethylene-Inducing Xylanase Receptor 2 (EIX2), and with the closest tomato homologs of Arabidopsis thaliana (At) Clavata 2 (CLV2) and Too Many Mouths (TMM) (Liebrand et al., 2013).

Later, many more RLPs were found to constitutively interact with, and depend on, SOBIR1 for their function. Examples are tomato Cuscuta Receptor 1 (CuRe1), which is involved in resistance to the parasitic plant Cuscuta reflexa (Hegenauer et al., 2016); the tomato Immunity (I) protein, which recognizes Avr1 from Fusarium oxysporum f. sp. lycopersici (Fol) (Catanzariti et al., 2017); tomato Elicitin Response (ELR), which recognizes INF1 elicitin of Phytophthora infestans (Domazakis et al., 2018); BnLepR3, which is an RLP from Brassica napus required for resistance to Leptosphaeria maculans (Ma \& Borhan, 2015); and RXEG1 from Nicotiana benthamiana (Nb), which recognizes the widely occurring microbial glycoside hydrolase 12 protein (XEG1) (Wang et al., 2018b). Furthermore, for several RLPs from Arabidopsis thaliana (hereafter referred to as Arabidopsis) constitutive interaction with, and functional dependence on, SOBIR1 has been reported, including RLP1 (ReMAX) (Jehle et al., 2013), RLP23 (Bi et al., 2014), RLP30 (Zhang et al., 2013), and RLP42 (RBPG1) (Zhang et al., 2014). The only RLP so far found that seems not to depend on SOBIR1 for signalling is the ColdShock Protein Receptor of N. benthamiana (NbCSPR) (Saur et al., 2016). Saur and coworkers showed that NbCSPR recognizes the Cold-Shock Protein 22 (csp22) peptide derived from bacterial cold-shock protein, and that NbCSPR interacts with SOBIR1 (Saur et al., 2016). Curiously, SOBIR1 was found not to be essential for NbCSPR function. Interestingly, quickly after the study of Saur and co-workers was published, the RLK Cold-Shock Protein Receptor (NbCORE) was also found to be required for csp22 perception (Wang et al., 2016). NbCORE is an RLK, which might explain why SOBIR1 does not play a role in csp22 signalling (Wang et al., 2016). 
The recruitment of BAK1 upon ligand recognition has been shown for RLKs, but recently, also RLPs have been shown to recruit this co-receptor to initiate signalling (Fig. 2) (Albert et al., 2015; Postma et al., 2016; Wang et al., 2018b; Domazakis et al., 2018). Upon ligand recognition by the RLP, BAK1 is recruited to the RLP/SOBIR1 complex to enable downstream signalling (Albert et al., 2015; Postma et al., 2016). This renders BAK1 recruitment a key step in defence initiation upon extracellular ligand recognition by both RLKs and RLPS (Fig. 2).

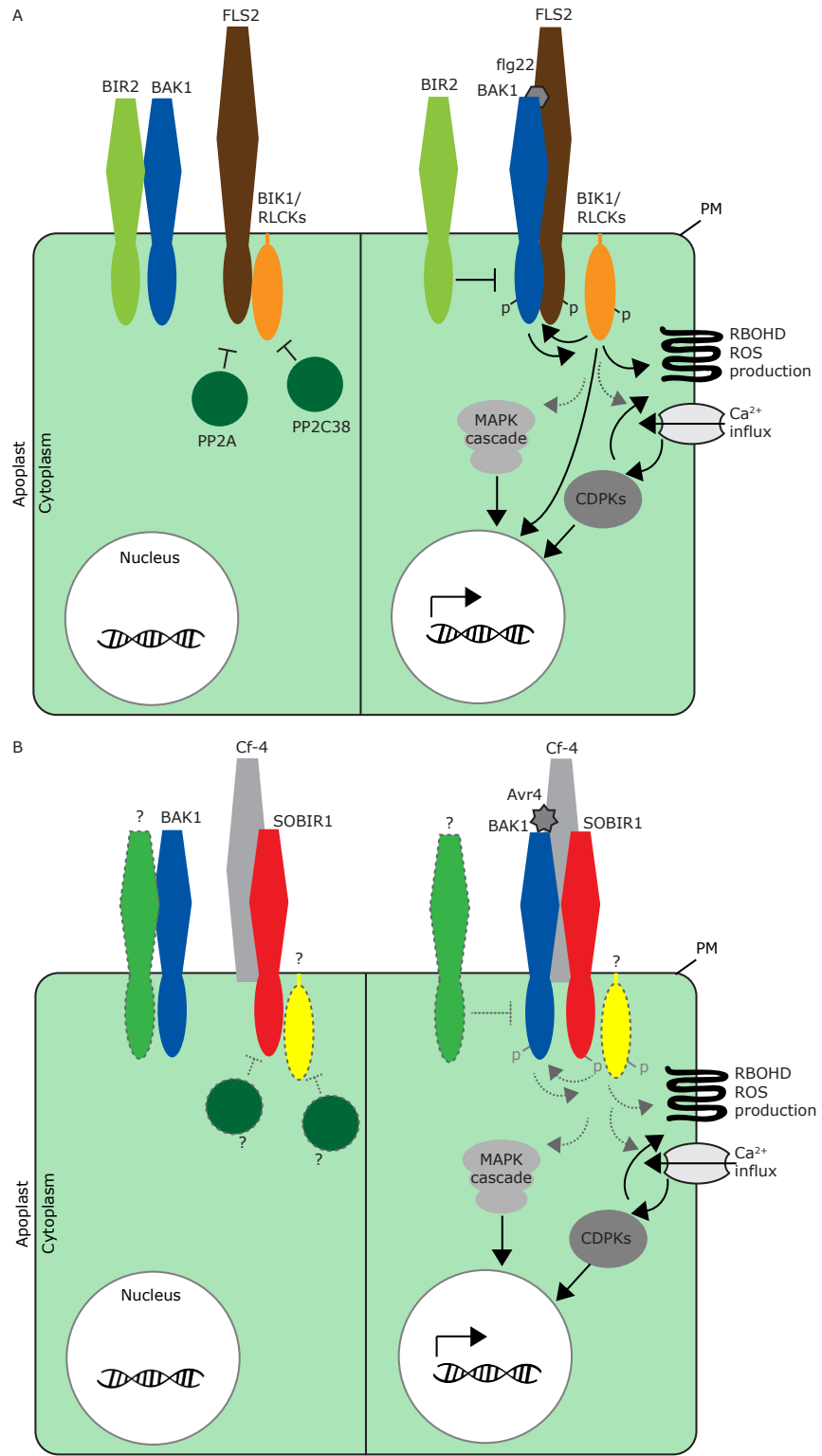


Figure 2. Schematic overview of the activation of immune responses by TM-receptors that are present on the PM. (A) Current model of immune signalling downstream of RLKs, taking FLS2 as an example. In the resting state (left), FLS2 (brown) interacts with BIK1 (orange) and their activity is kept in check by phosphatases (dark green circles). BAK1 (blue) is sequestered from the FLS2/BIK1 complex by BIR2 (light green). Upon flg22 recognition by FLS2 (right), BAK1 is recruited to the FLS2/BIK1 complex, and transphosphorylation events take place between BAK1, FLS2, and BIK1 (arrows). Subsequently, phosphorylated BIK1 is released and activates ROS production by phosphorylating PM-localised RBOHD. The defence signal is amplified through CDPKs, as a result of a strong $\mathrm{Ca}^{2+}$ influx. Next, the activated MAPK cascade and CDPKs reprogram transcription via the activation of transcription factors by phosphorylation, among which are the so-called WRKYs, to switch on the expression of defence-related genes. How MAPKs are precisely activated is not clear yet, but possibly certain RLCKs are involved in this process. (B) Current model of immune signalling downstream of RLPs, taking Cf-4 as an example. Cf-4 (grey) constitutively interacts with SOBIR1 (red). In the resting state (left), BAK1 is probably sequestered by a receptor-like (pseudo-) kinase (green). SOBIR1 likely interacts with an RLCK (yellow), and both kinases are expected to be kept in check by phosphatases (dark green circles). Upon ligand (Avr4) recognition (right), BAK1 is recruited to the $\mathrm{Cf}$-4/SOBIR1-complex, and probably transphosphorylation events take place between BAK1, SOBIR1, and a hypothetical RLCK. The precise downstream steps are not clear yet, but ROS are produced and transcription is reprogrammed to activate defence gene expression, likely via MAPKs and CDPKs. Anticipated signalling proteins and pathways are indicated with question marks, dotted grey lines, and grey arrowheads. FLS2, Flagellin-Sensing 2; BIK1, Botrytis-Induced Kinase-1; BAK1, BrassinosteroidInsensitive 1-Associated Kinase 1; BIR2, BAK1-Interacting RLK 2; flg22, flagellum bacterial peptide 22; ROS, reactive oxygen species; RBOHD, Respiratory Burst Oxidase Homologue D; MAPK, mitogen-activated protein kinase; $\mathrm{CDPK}$, $\mathrm{Ca}^{2+}$-dependent protein kinase; $\mathrm{Cf}-4$, $\mathrm{C}$. fulvum resistance gene mediating recognition of Avr4; SOBIR1, Suppressor Of BIR1 - 1; RLCK, receptor-like cytoplasmic kinase; Avr4, Avirulence 4; WRKYs, WRKY transcription factors.

\section{Sensing 'the ins'}

Inside the plant cell, nucleotide-binding (NB)-LRR receptors allow the detection of intracellular effector proteins from pathogens (Fig. 1A), or the effect that these proteins have on virulence targets (Dodds \& Rathjen, 2010; Win et al., 2012; Jones et al., 2016; Białas et al., 2017; Kourelis \& van der Hoorn, 2018). Several pathogens deliver effectors into the host cells, for instance bacteria via their type-threesecretion system (TTSS), and fungi and oomycetes through their haustoria, which are specialised feeding structures. Pathogens deliver these effector proteins into the plant cell to manipulate the host, and thereby suppress or circumvent defence responses (Dou \& Zhou, 2012). Subsequent recognition of these effectors by NBLRRs initiates a cascade of responses leading to immunity (Zhang et al., 2017). Next to direct recognition, diverse additional molecular mechanisms underlying the triggering of immunity have been unravelled. These mechanisms include guarding of effector virulence targets by NB-LRRs and decoys for effectors fused to NB-LRRs (Jones et al., 2016; Cesari, 2017; Kourelis \& van der Hoorn, 2018). 


\section{BOX1: The evolutionary battle between plants and pathogens}

The constant battle between plants and pathogens, with an alternation between plant resistance (pathogen avirulence) and plant susceptibility (pathogen virulence), manifests itself as a molecular arms race between plants and pathogens (van der Hoorn et al., 2002; Kourelis \& van der Hoorn, 2018). This co-evolution has given rise to an abundance of plant receptors that enable recognition of pathogen presence. Furthermore, due to this co-evolution a wide range of physiological races (strains) of pathogens evolved, that carry differential sets of effector genes aimed to avoid recognition or suppress plant defence responses.

Conserved structural components of invading pathogens (MAMPs), and the potential damage that they cause resulting in the release of DAMPs, form a first source of targets to be recognised by the host plant. Recognition of these MAMPs and DAMPs in the apoplast leads to the activation of defence responses, traditionally referred to as MAMP-triggered immunity (MTI), resulting in basal plant resistance (Jones and Dangl, 2006). This recognition is achieved by pattern recognition receptors (PRRs), located on the plasma membrane (PM). To combat the effect of this initial recognition, specialised pathogens have evolved effector proteins to suppress or avoid MTI-related responses, leading to effector-triggered susceptibility (ETS). Subsequent evolution of additional host receptors that specifically recognize these effectors or their manipulated effector targets, in its turn leads to effector-triggered immunity (ETI). To combat these host receptors, pathogens adjust their effector repertoire to avoid ETI, and employ alternative effectors to again cause ETS.

Ongoing research has revealed that the separation between MAMPs and effectors, as well as the distinction between MTI and ETI, is less clear than initially proposed (Boller \& Felix, 2009; Thomma et al., 2011). In fact, the characteristics of these invasion-derived compounds and the responses that they initiate form a kind of continuum (Boller \& Felix, 2009; Thomma et al., 2011; Cook et al., 2015). Therefore, in the recently proposed invasion model, MAMPs, DAMPs, and effectors have all been postulated to be so-called invasion patterns (IPs) (Cook et al., 2012).2015). These IPs can be extracellular as well as intracellular, and are recognized by IP receptors (IPRs), leading to IP-triggered responses (IPTRs) (Cook et al., 2015). In this thesis, I will mainly use the term transmembrane (TM)-receptor for receptors that sense extracellular IPs. The term TM-receptor encompasses PRRs, which sense MAMPs and DAMPs (historically classified to be involved in MTI), as well as immune receptors, which sense extracellular effectors (historically classified to be involved in ETI).

Despite the lack of an adaptive immune system in individual plants, as compared to mammals (as far as we know), the evolutionary battle between pathogen and host has led to the acquisition of a plethora of receptors by plants (van der Hoorn et al., 2002; Lehti-Shiu et al., 2009; Jacob et al., 2013). Regarding TM-receptors, the model plant Arabidopsis thaliana (Arabidopsis) for example carries around 450 genes encoding RLKs, of which 200 are LRR-RLKs (Lehti-Shiu et al., 2009; Fischer et al., 2016). In a similar range, tomato has around 500 genes encoding RLKs, of which 250 are LRR-RLKs (Sakamoto et al., 2012; Andolfo et al., 2013). There are 57 genes encoding LRR-RLPs in the genome of Arabidopsis, and around 150 and 90 of them are present in the genomes of tomato and rice, respectively (Wang et al., 2008; Andolfo et al., 2013; Fritz-Laylin et al., 2005). Resistance genes have likely expanded via duplication and diversification, resulting in tandem arrays of homologous, but distinct, receptors that are able to recognize a wide range of different effectors (Parniske et al., 1997; Thomas et al., 1998; Rivas \& Thomas, 2005; van der Hoorn et al., 2002; Stam et al., 2017). On the other hand, as a result of selection pressure, pathogens loose and evolve effectors to provide novel ways of attacking plants and avoiding recognition (Dou \& Zhou, 2012; Tang et al., 2017; Khan et al., 2018). The biotrophic fungus C. fulvum, for instance, has evolved specific effectors to block or sequester chitin from degradation and recognition, respectively, thereby promoting its virulence (van den Burg et al., 2006; van Esse et al., 2007; Bolton 2008; de Jonge et al., 2010). Not only fungal pathogens, but also oomycete and bacterial pathogens have evolved an impressive effector repertoire as a result of this continuous arms race (Schornack et al., 2009; Deslandes \& Rivas, 2012). Plants, in their turn, will continue to ward off pathogens with novel receptors that emerge as a result of this ongoing co-evolution. 


\section{BOX2: The roles of TM-receptors in development}

Plant TM-receptors are not only essential for successful defence against invading pathogens, but also for proper growth and development (de Smet et al., 2009; Wang et al., 2010). Many developmental processes of plants are under control of TM-receptors, for example the maintenance of the plant epidermis and the shoot and root apical meristem, and the development of stomata, vascular tissue, and anthers (de Smet et al., 2009; Wang et al., 2010).

A well-known example of an RLK that functions in development is Brassinosteroid-Insensitive 1 (BRI1) from Arabidopsis (Li \& Chory, 1997). BRI1 senses brassinosteroids (BRs), and initiates downstream signalling to regulate growth and development. Similar to defence signalling by RLKs, this signalling mechanism also includes the recruitment of SERKs, and also several RLCKs play a role in downstream signalling (Li et al., 2002; Nam \& Li, 2002; Tang et al., 2008; Eckardt, 2011; Liang \& Zhou, 2018).

Another RLK from Arabidopsis involved in development is Clavata 1 (CLV1) (Clark et al., 1997). CLV1 binds the plant-derived signalling peptide CLV3 (Ogawa et al., 2008). There are different parallel signalling pathways triggered by CLV3, one of them involves the constitutive complex CLV2/ Coryne (CRN), which forms heterodimeric complexes with and without CLV1 (Jeong et al., 1999; Pan et al., 2016). CLV2 is an RLP, and CRN has been reported to be a pseudokinase, without a clear ectodomain (Müller et al., 2008; Bleckman et al., 2010).

Another well-known example of an RLP playing a role in development is Too Many Mouths (TMM), which is involved in stomatal development (Geisler et al., 2000; Nadeau \& Sack, 2002). TMM interacts with RLKs of the ERECTA family to initiate signalling (Shpak et al., 2005; Lee et al., 2012). Surprisingly, CLV2, as well as TMM, appear to interact with the regulatory RLK SOBIR1 (Liebrand et al., 2013). Interestingly, these RLPs not only play a role in development, but also in defence (Pan et al., 2016; Jordá et al., 2016). This again highlights the entangled pathways of development and defence. The biological relevance of the observed interaction between the RLPS TMM and CLV2 with SOBIR1 remains to be elucidated.

Interestingly, before SOBIR1 was found to interact with RLPs involved in defence, this regulatory RLK was already found to play a role in floral organ shedding in Arabidopsis (Leslie et al., 2010). Organ shedding is regulated via the perception of the plant-derived signalling peptide Inflorescence Deficient in Abscission (IDA) by the RLKs Haesa (greek for 'to adhere to', HAE) and Haesa-like 2 (HSL2), together with the co-receptor SERK1 (Niederhuth et al., 2013; Santiago et al., 2016). In these reports, SOBIR1, here referred to as Evershed (EVR), was found to inhibit floral organ shedding, as a knockout of EVR (containing a mutation that inactivates the EVR kinase domain) in a nevershed (NEV) knockout background, restored floral organ shedding (Leslie et al., 2010). In this system, NEV, an ADP-Ribosylation Factor-GTPase-Activating Protein (ARF-GAP), and EVR are proposed to play a role in sub-cellular trafficking of receptor complexes (Liljegren, 2012; Gubert \& Liljegren, 2014). NEV promotes the recycling of HAE/HSL2 receptors to the PM, and on the contrary, EVR promotes the removal of HAE/HSL2 receptors from the PM (Liljegren et al., 2009; Leslie et al., 2010). Interestingly, the RLCK Cast Away (CST) has also been shown to promote the removal of HAE/HSL2 receptors from the PM together with EVR, and thereby inhibit floral organ shedding in Arabidopsis (Burr et al., 2011; Groner et al., 2016).

Together, these studies demonstrate that several TM-receptors play a role in development and defence, and that these functions are tightly entangled. 


\section{Downstream defence responses triggered by activated plant receptors}

\section{Downstream defence responses triggered by TM-receptors}

The defence signalling pathway that is being activated, starting from the event of pathogen recognition in the apoplast and ending, if successful, at the actual state of disease resistance, consists of an impressive cascade of actions, resembling a domino game with many amplification steps (Fig. 2). In this cascade, the recognition of extracellular IPs by TM-receptors is first of all followed by the recruitment of (co-) receptors to the activated TM-receptor (Couto \& Zipfel, 2016; Tang et al., 2017; Hohmann et al., 2018). This complex formation is a common first step after ligand recognition in signalling for immunity. As mentioned before, BAK1 forms a complex with for instance FLS2 upon flg22 recognition (Fig. 2A) (Chinchilla et al., 2007; Heese et al., 2007). The chitin receptor Chitin Elicitor Receptor Kinase 1 (CERK1) also gets involved in a higher order complex, as it dimerizes with the Lysin-motif Receptor Kinase 5 (LYK5) upon chitin recognition (Cao et al., 2014). Receptor homo- or heterodimerization enables the interacting partners to initiate further downstream signalling, involving the activation of cytoplasmic signalling partners. The formation of active signalling complexes at the PM is also a common first step in signalling for development, and several proteins, e.g. BAK1, have been shown to play a role in signalling for immunity as well as development (BOX 2).

A key step connecting an activated RLK at the cell surface with an intracellular response is facilitated by receptor-like cytoplasmic kinases (RLCKs) (Fig. 2A) (Zhang et al., 2010; Lu et al., 2010; Liang \& Zhou, 2018). RLCKs consist of a kinase domain with a myristoylation site, carrying a fatty acid that forms an anchor to the plasma membrane (PM). A well-studied RLCK is Arabidopsis Botrytis Induced Kinase-1 (BIK1) (Veronese et al., 2006), which in the resting state interacts with FLS2 (Zhang et al., 2010; Lu et al., 2010). Upon elicitation by flg22, BAK1 is recruited, and BIK1, FLS2, and BAK1 transphosphorylate each other (Fig. 2A) (Lu et al., 2010; Zhang et al., 2010). Subsequently, BIK1 is released from the complex to initiate further signalling (Li et al., 2014; Kadota et al., 2014). The large variety of RLCKs and their roles downstream of several TM-receptors, make this family a key regulatory component in immune signalling (Couto \& Zipfel, 2016; Tang et al., 2017; Liang \& Zhou, 2018).

Fast intracellular responses upon extracellular ligand recognition include the instant elevation of cytoplasmic $\mathrm{Ca}^{2+}$ levels as a result of the opening of $\mathrm{Ca}^{2+}$ channels, and the production of reactive oxygen species (ROS) (Fig. 2A). BIK1 has been shown to regulate ROS production downstream of RLKs via the activation of Respiratory Burst Oxidase Homologue D (RBOHD) (Kadota et al., 2014; Li et al., 2014). RBOHD, which is responsible for ROS production, is directly phosphorylated by 
activated BIK1 upon flg22 recognition (Fig. 2A). RLCKs are likely to be also involved in regulating $\mathrm{Ca}^{2+}$ levels, but this remains elusive. Downstream of FLS2, activated BIK1 is tightly regulated via proteasomal degradation. It has for example been shown that heterotrimeric $\mathrm{G}$ proteins and E3 ligases dynamically regulate the degradation of BIK1 depending on its activation state, to ensure full signalling capacity (Liang et al., 2016; Wang et al., 2018a). Just recently, it was shown that Brassinosteroid Signalling Kinase 1 (BSK1), which is another RLCK downstream of TM-receptors, provides a direct link from the PM to the mitogen-activated protein kinase (MAPK) cascade, as BSK1 transphosphorylates MAP kinase-kinase-kinase 5 (MAPKKK5) upon flg22 elicitation (Yan et al., 2018).

$\mathrm{Ca}^{2+}$-dependent protein kinases (CDPKs) and MAPKs provide signalling links from the cytoplasm to the nucleus, and the transcriptional reprogramming that takes place therein (Fig. 2A) (Seybold et al., 2014; Lee et al., 2015; Zhang et al., 2018). CDPKs sense the change in $\mathrm{Ca}^{2+}$ concentration and activate transcription factors that move to the nucleus for defence gene regulation (Seybold et al., 2014). The MAPK cascade includes several MAPKKKs, MAPKKs, and MAPKs that phosphorylate each other, resulting in an amplification of the initial signal. This pathway is essential for defence gene expression, as it mediates the activation of so-called WRKY transcription factors that regulate defence gene expression upon activation of immune signalling (Lee et al., 2015; Li et al., 2016a; Birkenbihl et al., 2017). Upregulation of the expression of large sets of defence-related genes will eventually contribute to mounting resistance, amongst others through the production of antimicrobial compounds, fungal cell wall-degrading enzymes like glucanases and chitinases, and phytohormones such as salicylic acid (Pieterse et al., 2012). Interestingly, a recent study shows a direct link between BIK1 and WRKY transcription factors, as BIK1 was shown to be able to transphosphorylate certain WRKYs and thereby regulate the production of phytohormones (Lal et al., 2018).

\section{Downstream defence responses triggered by cytoplasmic receptors}

Pathogens of which the effectors reach the cytoplasm of the cells of the host plant, are confronted with recognition by cytoplasmic NB-LRR receptors (Fig. 1A). Direct or indirect recognition leads to activation and dimerization of NB-LRRs, to the formation of higher order complexes with additional signalling partners, and to subsequent initiation of downstream signalling (Takken \& Goverse, 2012; Jones et al., 2016; Wu et al., 2017; Zhang et al., 2017). NB-LRRs can target the DNA in the nucleus of the host cells (Fenyk et al., 2015). However, currently it is not known whether this DNA binding results in transcriptional reprogramming, or in DNA damage that in its turn causes local programmed cell-death, referred to as the Hypersensitive Response (HR), and resistance. 


\section{All signalling steps that lead to defence are entangled}

Interestingly, all signalling steps leading to defence initiation seem to be entangled. For instance, $\mathrm{Ca}^{2+}$ activates not only CDPKs, but also promotes RBOHD enzyme activity, resulting in an amplification of the response and the activation of multiple defence-related processes at the same time (Kadota et al., 2015).

Furthermore, NB-LRRs are, next to sensing invading pathogens, also involved as 'helper' proteins in immune signalling, and help so-called 'sensor' NB-LRRs to be functional (Wu et al., 2017). Possibly, NB-LRRs are also supporting the transduction of signalling steps that are initiated by TM-receptors at the PM (Gabriels et al., 2007).

Different steps in defence pathways regulate each other, and a perturbation of this regulation might have huge consequences and can result in an unbalanced signalling output that for example causes constitutive immunity. It is essential to keep these connections in mind when studying immune pathways. For example, if the knockout of a certain gene results in strong (constitutive) activation of defence responses, this gene might code for a negative regulator of defence. However, it is also possible that the encoded protein is actually a positive regulator of defence, and that its integrity is guarded by a host protein, leading to the activation of immune responses when this positive regulator is knocked-out (Couto \& Zipfel, 2016; Veronese et al., 2006).

\section{The delicate balance of regulating plant immunity in relation to development}

It is important to maintain a tight balance between defence and development, as investing in defence is energy-demanding. Additionally, unintentionally activating defence when it should not be activated, can be an expensive mistake, and may cause growth defects and even plant death (Lorrain et al., 2003; Belkhadir et al., 2014; Lozano-Durán \& Zipfel, 2015; Cao, 2016; Couto \& Zipfel, 2016; Smakowska et al., 2016). Furthermore, many plant TM-receptors have overlapping roles in immunity and development (BOX2). For instance BAK1 is a co-receptor for immune receptors like FLS2, but also for the BR receptor BRI1 (Li et al., 2002; Nam \& Li, 2002; Chinchilla et al., 2007; Heese et al., 2007; LozanoDuran \& Zipfel, 2015; Yasuda et al., 2017). SOBIR1, in developmental studies referred to as Evershed (EVR), has been shown to inhibit floral organ shedding in Arabidopsis, by regulating the trafficking of receptors that play a role in this process (BOX 2) (Leslie et al., 2010). Also several RLCKs have been shown to be involved in defence, as well as in BR signalling. For instance BIK1 is a positive regulator of FLS2-triggered defence responses, but a negative regulator of BR signalling (Lu et al., 2010; Zhang et al., 2010; Lin et al., 2013; Eckardt, 2011). Through breeding and selection to obtain high yields in agriculture, the maintenance of broad spectrum resistance to pathogens has historically been overlooked. Wild relatives of our crop species often have smaller fruits and produce 
less biomass, but also show stronger basal defence. So, as there is a growth-defence trade-off for plants, defence activation should only occur when necessary, and energy should not be spent on defence when there are no pathogens around.

Maintaining the balance between defence and development is coordinated at several levels (Couto \& Zipfel, 2016). One level to control defence responses is the regulation of the amount of TM-receptors that is available to trigger defence responses. The biogenesis of functional TM-receptors, which involves proper folding and decorating, including glycosylation, in the endoplasmic reticulum (ER), is a demanding task (Li et al., 2009; Liebrand et al., 2012; Sun et al., 2014). TM-receptor production is less efficient when the plant has to put more effort in development and survival, for instance under high temperature conditions (de Jong et al., 2002; Stulemeijer, 2008). Next to the biogenesis of TM-receptors, their delivery at the PM, their recycling, and their degradation are essential components of the regulation of defence responses (Postma et al., 2016; Frescatada-Rosa et al., 2015; Ben Khaled et al., 2015; Liang et al., 2016; Mbenguea et al., 2016).

When present at the PM, the activity of TM-receptors is, amongst others, regulated by influencing their spatial separation and hetero-dimerization with co-receptors (Fig. 2A) (Couto \& Zipfel, 2016; Bücherl et al., 2017; Hohmann et al., 2017). Dimerization with signalling partners is an important step in defence signalling, and blocking this interaction by sequestering co-receptors in the resting state inhibits defence activation (Shaw et al., 2014). The pseudokinase BAK1-Interacting RLK 2 (BIR2), for example, has been shown to interact with BAK1 in the resting state (Halter et al., 2014a and 2014b). Upon ligand perception, BAK1 is released from BIR2 and thereby becomes available to form active immune complexes. Spatial separation in nanoclusters on the PM also accounts for specificity and regulation of immune complexes (Hutten et al., 2017; Hao et al., 2014; Wang et al., 2015; Jarsch et al., 2014; Somssich et al., 2015; Bücherl et al., 2017). BAK1 is a co-receptor for many TM-receptors that play a role in defence and development, and seems to be present in distinct nanoclusters, each responsible for specific signalling outputs (Bücherl et al., 2017).

After complex formation with co-receptor(s), both in defence and development a signalling cascade is initiated in which phosphorylation is a crucial step (Gish \& Clark, 2011; Macho et al., 2015). For instance, BAK1, FLS2, and BIK1 transphosphorylate each other upon flg22 perception (Lu et al., 2010; Zhang et al., 2010). In its turn, dephosphorylation by phosphatases is essential to keep the activity of immune receptors in check. For example, in the resting state, BAK1 and BIK1 are kept in check by Protein Phosphatase 2A (PP2A) and PP2C38, respectively (Segonzac et al., 2014; Couto et al., 2016). This control step, in these cases consisting of de-phosphorylation by a PP2C member, has also been shown for the rice RLK Xa21 conferring resistance to the bacterial pathogen Xanthomonas oryzae pv oryzae secreting Ax21 (Park et al., 2008). 
As mentioned before, the integrity of TM-receptors is likely guarded by cytoplasmic NB-LRRs (Couto \& Zipfel, 2016). BAK1, for instance, has been identified as a positive regulator of defence, but also as a negative regulator, as overexpression of BAK1, but also the knockout of BAK1, causes the onset of defence responses (Domínguez-Ferreras et al., 2015; Liu et al., 2016). It is likely that BAK1, and other TM-receptors, not only signal for defence when sensing a pathogen, but are also guarded by cytoplasmic NB-LRRs to secure the initiation of defence responses upon manipulation of these TM-receptors by cytoplasmic effectors.

Downstream responses after TM-receptor activation include ROS accumulation, MAPK activation, transcriptional reprogramming, and the production of phytohormones such as salicylic acid (SA). Each of these steps is also likely to be negatively regulated in order to maintain plant homeostasis when there is no pathogen pressure (Bigeard et al., 2015; Li et al., 2016a; Li et al., 2016b; Couto \& Zipfel, 2016).

\section{The Cladosporium fulvum - tomato pathosystem}

Often, plant-pathogen interactions are studied using the model plant Arabidopsis. These studies have gained tremendous amounts of knowledge on the molecular mechanisms behind plant resistance and pathogen virulence. However, it is important to not only obtain fundamental knowledge from this Brassicaceous plant, but also from Solanaceous plants like tomato. Research on defence mechanisms in Solanaceous plants can potentially lead to faster applicable knowledge concerning a large range of crop plants such as tomato, pepper, and potato. Nicotiana benthamiana and $N$. tabacum are also Solanaceous plants, and these are often used in laboratories as model plants to represent the family of Solanaceous plants, because they facilitate the execution of relatively easy and fast experiments (Goodin et al., 2008).

The pathosystem Cladosporium fulvum - tomato has been a key system to study plant immunity for more than 40 years (de Wit et al., 1977; Joosten \& de Wit 1999; Stergiopoulos \& de Wit 2009; de Wit, 2016). C. fulvum is an asexual, non-obligate biotrophic fungus, with Solanum lycopersicum ( $S$, tomato), including some wild Solanum varieties, as its only known host. Germ tubes of fungal conidia enter the extracellular space of the leaves of tomato plants via stomata at the abaxial side of the leaves (Thomma et al., 2005). The mycelium eventually colonises the extracellular space between the cells (Thomma et al., 2005). Infection may lead to symptoms like yellow spots, white to brown mould, and wilting of the leaves (Thomma et al., 2005). Several proteins are secreted by $C$. fulvum into the extracellular space to promote infection. Well-studied examples of such extracellular proteins are Avirulence protein 2 (Avr2) (Luderer et al., 2002), Avr4 (Joosten et al., 1994), Avr4E (Westerink et al., 2004), 
Avr5 (Mesarich et al., 2014), Avr9 (van den Ackerveken et al., 1992), and Extra Cellular Protein 6 (ECP6) (Bolton et al., 2008). This effector repertoire has different modes of promoting C. fulvum virulence, for instance by protecting chitin present in the cell walls of C. fulvum from being degraded by plant chitinases (Avr4) (van den Burg et al., 2006), sequestering or blocking chitin fragments released by $C$. fulvum from recognition by the plant (Ecp6) (de Jonge et al., 2010), or inhibiting the plant cysteine protease referred to as Required for Cladosporium Resistance 3 (Rcr3) by Avr2 (Rooney et al., 2005; van Esse et al., 2008). Due to the presence of homologues of at least some of these effector proteins in other fungal species, it is debatable whether to call them true effectors or MAMPs (Kohler et al., 2016; Mesarich et al., 2015; Bolton et al., 2008; Thomma et al., 2011; Stergiopoulos et al., 2010).

Many effectors of $C$. fulvum are not only virulence factors, but also act as avirulence factors, meaning that these proteins are specifically recognized by tomato genotypes that are resistant to the pathogen. Tomato has evolved a broad set of RLPs to recognize Avrs, leading to defence activation in a gene-for-gene fashion (de Wit, 1995). These RLPs, conferring resistance to $C$. fulvum, are referred to as $C f$ proteins and localize at the PM (Piedras et al., 2000; Postma et al., 2016). The first cloned Cf gene is Cf-9, and the encoded Cf-9 protein was shown to specifically recognize Avr9 (Jones et al., 1994). Hereafter, more $\mathrm{Cf}$ proteins recognizing specific $C$. fulvum effectors were discovered, namely Cf-2 (Dixon et al., 1996), Cf-4 (Thomas et al., 1997), Cf-4E (Takken et al., 1999), and Cf-5 (Dixon et al., 1998). Direct recognition of Avrs by receptors is one mechanism of plants to recognize pathogens, for which the $C f-4 / A v r 4$ gene-for-gene pair is anticipated to be an example (see next paragraph). Interestingly, the mechanism by which Avr2 is recognized by Cf- 2 appears to typically follow the guard model, as Cf-2 recognizes the inhibitory effect that Avr2 has on the extracellular tomato protease Rcr3 (Rooney et al., 2005). Cf-2 guards Rcr3, and the interaction between Avr2 and Rcr3, probably resulting in a conformational change of the protease, is recognized by $\mathrm{Cf}-2$, which then triggers immunity. The continuous evolution of the RLP family provides a toolbox for additional resistance traits (BOX 1).

\section{Cf-4 and Avr4, a model gene-for-gene pair}

One of the main model gene-for-gene pairs of the $C$. fulvum - tomato pathosystem is Cf-4/Avr4. The avirulence gene Avr4 of C. fulvum was cloned almost 25 years ago (Joosten et al., 1994), and since then, this effector has been studied intensively. Avr4 is a small cysteine-rich protein with four disulphide bridges, is only produced upon plant infection, and accumulates in the apoplast of the host plant (Joosten et al., 1994; Joosten et al., 1997; van den Burg et al., 2003). Avr4 has been found to bind chitin, and prevent fungal hyphae from degradation by chitinases, thereby promoting fungal virulence (van den Burg et al., 2003 and 2006; van Esse et al., 2007). 
The TM-receptor $\mathrm{Cf}-4$ has been cloned from the wild tomato species Solanum habrochaites (formerly called Lycopersicon hirsutum), and is an RLP with an ECD that comprises 25 LRRs (Thomas et al., 1997 and 1998). Cf-4 is able to recognize Avr4, and confers resistance to Avr4-secreting strains of C. fulvum (Thomas et al., 1997). The evolution of an instable form of Avr4, in which one disulphide bridge is lost due to a single point-mutation in the encoding gene, has been shown effective to circumvent recognition by the host, but still maintain the intrinsic function of fungal hyphae protection (Joosten et al., 1994; Joosten et al., 1997; van den Burg et al., 2006). This instable form of Avr4 has lost its avirulence function, but maintained its virulence function as it still binds to fungal chitin upon its secretion, but is probably too instable to be detected by the Cf- 4 receptor at the PM of the host (Joosten et al., 1997; van den Burg et al., 2006).

Interestingly, homologues of $C$. fulvum Avr4 have been found in other fungal species and were shown to also bind fungal chitin (Stergiopoulos et al., 2010; Mesarich et al., 2015; Kohler et al., 2016). The Avr4 homologues of Mycosphaerella fijiensis, Dothistroma septosporum, and Pseudocercospora fuligena (Pf) are all recognized by Cf-4 and trigger an HR (Stergiopoulos et al., 2010; Mesarich et al., 2015; Kohler et al., 2016). The crystal structure of PfAvr4 even revealed the role of specific amino acids in chitin binding (Kohler et al., 2016). The wide occurrence of Avr4 homologues allows to argue that Avr4 qualifies as a MAMP and that $\mathrm{Cf}$-4-mediated recognition of Avr4 leads to MTI (Thomma et al., 2011).

Avr4 protects the pathogen against degradation by plant chitinases and is thought not to have a virulence target in the plant. Infiltration of Avr4 protein produced by Pichia pastoris, as well as Avr4 overexpression via agroinfiltration, induces an $\mathrm{HR}$ in Cf4-carrying plants (van der Hoorn et al., 2000; van den Burg et al., 2001). These findings lead to assume that recognition of Avr4 by Cf-4 is probably direct (Mesarich et al., 2015; Kohler et al., 2016). However, so far no direct interaction between Cf-4 and Avr4 has been experimentally shown.

\section{Cf proteins require SOBIR1 for their functionality}

Until a few years ago, it has remained enigmatic how Cf proteins, which are RLPs without an intracellular kinase domain, signal for defence upon ligand recognition. However, as was hypothesized already more than 20 years ago (de Wit, 1995; Joosten \& de Wit, 1999), an RLK (namely SOBIR1) was recently found to constitutively interact with Cf proteins (Liebrand et al., 2013). This constitutive interaction with SOBIR1 is required for the accumulation and function of Cf proteins (Liebrand et al., 2013 and 2014). Later, the interaction with SOBIR 1 and the requirement of SOBIR 1 for the biological function was also found for many other RLPs, as described earlier. These observations support that constitutively interacting with SOBIR1 is a common mechanism to form bimolecular RLKs from RLPs (Liebrand et al., 2014; Gust \& Felix, 2014). 
SOBIR1 was initially identified as a 'Suppressor Of BIR1-1' in a mutant screen by Gao and co-workers (2009). They concluded that SOBIR1 is a positive regulator of defence, as the overexpression of SOBIR1 in Arabidopsis constitutively activates immunity. Knockout or silencing of SOBIR1 does not directly lead to a clear hypersusceptible phenotype (Goa et al., 2009; Leslie et al., 2010). This is probably due to the presence of multiple layers of plant defence that all contribute to resistance, in which also SOBIR1-independent RLKs and NB-LRRs play a role. Nevertheless, the role of SOBIR1 in resistance to bacteria, biotrophic and necrotrophic fungi, and oomycetes is starting to be unravelled (Gao et al., 2009; Liebrand et al., 2013; Zhang et al., 2013; Zhang et al., 2014; Albert et al., 2015; Peng et al., 2015; Postma et al., 2016; Wang et al., 2018b; Domazakis et al., 2018).

As was found for the typical PRR FLS2 (BOX 1), immune responses triggered upon ligand recognition by Cf proteins also include a ROS burst (May et al., 1996; Piedras et al., 1998), MAPK and CDPK activation (Stulemeijer et al., 2007; Romeis et al., 1999, 2000, and 2001), and massive transcriptional reprogramming through, amongst others, WRKY transcription factors (Fig. 2B) (Etalo et al., 2013). Strikingly, Cf genes are resistance genes that historically have been classified to be involved in ETI, as the encoded Cf receptors mediate the recognition of typical effectors (Avrs) and furthermore activate a defence response that is associated with a strong HR. In contrast, many other TM-receptors, like FLS2 and RLP23, are classified as PRRs involved in MTI (Jones and Dangl, 2006). To further unravel the similarities and differences between Cf-, RLP23-, and FLS2-mediated immunity, key steps in the cascade of signalling events that take place between effector recognition by $\mathrm{Cf}$ proteins and the final immune response, need to be elucidated (Fig. 2B).

Several groups have tried to identify signalling components that play a role downstream of Cf-proteins. VAP27 (Vesicle-Associated Protein 27) is likely to be involved in cellular trafficking of Cf-complexes (Laurent et al., 2000). In addition, ACIK1 (Avr9/Cf-9-Induced Kinase 1) is an RLCK of which the encoding gene was found to be upregulated during the Cf-9/Avr9-induced HR (Rowland et al., 2005). ACIK1 was shown to interact with CITRX (Cf-9-Interacting ThioRedoXin), a negative regulator of Cf-9-mediated defence responses (Rivas et al., 2004; Nekrasov et al., 2006). Recently, recruitment of the regulatory co-receptor BAK1 to the RLP/SOBIR1 complex was shown to take place as a first step upon ligand recognition by $\mathrm{Cf}-4$ and several other RLPs, including RLP23 (Postma et al., 2016; Albert et al., 2015; Wang et al., 2018b; Domazakis et al., 2018). Interestingly, Cf-4 triggers a strong HR upon recognition of the effector Avr4 (referred to as ETI), whereas RLP23 triggers much milder immune responses (referred to as $\mathrm{MTI}$ ) upon recognition of the nlp20 peptide derived from Necrosis and Ethylene-inducing Protein 1, which is a widely conserved MAMP (Böhm et al., 2014b). To understand the defence signalling mechanisms that are employed by RLPs, it is important to gain detailed insight into how BAK1 actually is recruited, 
whether transphosphorylation events between the kinase domains of SOBIR1 and BAK1 might actually play a role in initiating a downstream signal, reminiscent of FLS2/BAK1-triggered signalling, and what kind of additional cytoplasmic steps downstream of the activated RLP/SOBIR1/BAK1 complex, present at the cell surface, are taking place.

\section{Aims and outline of this thesis}

SOBIR1 is a central docking station for RLPs. SOBIR1 appears to act as a scaffold to stabilize RLPs and, in addition, its kinase activity is essential for downstream signalling. The work described in this thesis is aimed at unravelling the exact role of SOBIR1 in RLP-mediated defence signalling. Questions to be answered are: (1) does transphosphorylation of the kinase domains of SOBIR1 and BAK1 occur upon RLP activation by ligand recognition and subsequent BAK1 recruitment? (2) Is this transphosphorylation required to initiate downstream signalling? Furthermore, it is studied whether differential phosphorylation of the kinase domain of SOBIR1 might provide a specific signal for the activation of the immune response. Additionally, the identification and characterization of positive and negative regulators of the bimolecular Cf-4/SOBIR1 complex is pursued.

Immune receptors and their signalling partners are obvious virulence targets for pathogens, as their manipulation can suppress the activation of defence responses. Chapter 2 describes the suppression of SOBIR1-mediated immunity by the effector AvrPto of the bacterium Pseudomonas syringae pv tomato DC3000. Overexpression of AtSOBIR1 induces constitutive immunity in $N$. benthamiana. AvrPto interacts with SOBIR1, thereby suppressing AtSOBIR1-induced constitutive immunity, as well as the Cf-4/Avr4-triggered HR.

Chapter 3 describes the cooperation between SOBIR1 and BAK1 in immune signalling. BAK1 is recruited to RLP/SOBIR1 immune complexes upon activation by the matching ligand. SOBIR1 kinase activity is essential for defence signalling but not for its scaffold function, as kinase-dead SOBIR1 mutants are unable to signal for defence, but are still able to stabilize Cf-4. Here, it is shown that AtSOBIR1-induced constitutive immunity is BAK1-dependent. Moreover, phosphorylation of AtSOBIR1 appeared to be dependent on kinase-active BAK1, as well as on AtSOBIR1 kinase activity. This observation suggests that SOBIR1 first transphosphorylates BAK1, after which activated BAK1 transphosphorylates SOBIR1 to initiate downstream signalling for immunity.

Based on the results described in Chapter 3 it is likely that ligand recognition by an RLP, followed by BAK1 recruitment to the RLP/SOBIR1 complex, triggers a specific phosphorylation of SOBIR1. In Chapter 4, mutation analyses were performed, 
which revealed that three tyrosine and four threonine residues, present in the kinase domain of SOBIR1, are important for SOBIR1 functioning in immunity. Coimmunoprecipitation of SOBIR1, through a pull-down of Cf-4 in the resting state or Avr4-activated state, from N. benthamiana:Cf-4-eGFP plants, and subsequent analysis via mass spectrometry (MS), did not result in the identification of differential phosphorylation of the SOBIR1 kinase domain. However, immunoprecipitation of constitutively active AtSOBIR1 from $N$. benthamiana and subsequent analysis of the phosphorylation status of its kinase domain via MS, did show that several amino acids of this domain of SOBIR1 are phosphorylated in planta.

RLCKs are known to play an essential role in signalling downstream of PRRs, with for example BIK1 being an essential signalling component downstream of the activated FLS2/BAK1 complex. Several experimental approaches in Chapter 5 showed that AtBIK1 interacts with SOBIR1, meaning that this RLCK might also play a role in SOBIR1-mediated signalling. Phylogenetic analysis revealed several RLCKs in tomato and $N$. benthamiana as potential functional homologues of AtBIK1. Virus-Induced Gene Silencing (VIGS) of a subset of these BIK1 candidates in $N$. benthamiana did not identify individual RLCKs that might play a role downstream of the Cf-4/SOBIR1 complex, which is probably due to $R L C K$ gene redundancy. Split-luciferase analysis showed the interaction of several tomato RLCKs with SISOBIR1 and AtFLS2. Moreover, the RLCK SITPK1b (Tomato Protein Kinase $1 \mathrm{~b}$ ) was identified as a specific interactor of SISOBIR1, and therefore might play a role downstream of SOBIR1.

Physical separation of PRRs and their co-receptors on the PM by negative regulators, helps to keep signalling for defence in check, and allows to retain plant homeostasis concerning growth on the one hand and immunity on the other hand. BIR1, and its homologue BIR2, are negative regulators of immunity in Arabidopsis, and Chapter 6 focuses on the regulation of SOBIR1 signalling by these BIRs. Orthologues of BIR1 and BIR2 of tomato were identified, and their interaction with SIBAK1 was shown. Silencing of BIR1 in tomato leads to severe developmental effects, and is lethal in N. benthamiana. BIR1 silencing in tomato seems to sensitise the plant, as it appeared to slightly accelerate the Cf-4/Avr4-triggered HR. BIR2 silencing had no visible developmental effect, and also did not affect the intensity of the Cf-4/Avr4triggered HR. It was concluded that, also in Solanaceous plants, BIR1 is a negative regulator of cell death, and possibly a negative regulator of Cf-4/SOBIR1-mediated defence, through its interaction with SIBAK1. SIBIR2 appears not to be involved in the regulation of $\mathrm{Cf}-4 / \mathrm{SOBIR} 1$-mediated defence.

In Chapter $\mathbf{7}$ the results presented in this thesis are discussed, and form a basis to argue in favour of using an updated version of the invasion model. Signalling upon ligand recognition by LRR-containing TM-receptors, including RLKs as well as bimolecular RLP/SOBIR1-complexes, depends on similar downstream responses, 
including the recruitment and transphosphorylation of BAK1. This is underlining the blurred dichotomy between MTI and ETI. It is proposed to indeed abandon this distinction when describing plant-microbe interactions, as was already put forward in the invasion model. To provide a broadly including, but clearly distinguishing nomenclature, a spatial dichotomy is introduced. One should refer to all extracellular IPs as ExIPs, and to all intracellular IPs as InIPs. Recognition of ExIPs and InIPs then leads to extracellular-triggered immunity (ExTI) and intracellular-triggered immunity $(\ln T \mathrm{I})$, respectively. 


\section{References}

Akira S, Uematsu S, Takeuchi O. 2006. Pathogen recognition and innate immunity. Cell 124: 783-801.

Albert I, Böhm H, Albert M, Feiler CE, Imkampe J, Wallmeroth N, Brancato C, Raaymakers TM, Oome S, Zhang H, Krol E, Grefen C, Gust AA, Chai J, Hedrich R, Van den Ackerveken G, Nürnberger T. 2015. An RLP23-SOBIR1-BAK1 complex mediates NLP-triggered immunity. Nature Plants 1: 15140

Andolfo G, Sanseverino W, Rombauts S, Peer Y, Bradeen J, Carputo D, Frusciante L, Ercolano M. 2013. Overview of tomato (Solanum lycopersicum) candidate pathogen recognition genes reveals important Solanum R locus dynamics. New Phytologist 197: 223-237.

Belkhadir Y, Yang L, Hetzel J, Dangl JL, Chory J. 2014. The growth-defense pivot: crisis management in plants mediated by LRR-RK surface receptors. Trends in Biochemical Sciences 39: 447-456.

Ben Khaled S, Postma J, Robatzek S. 2015. A moving view: subcellular trafficking processes in pattern recognition receptor-triggered plant immunity. Annual Review of Phytopathology 53: 379-402.

Bi G, Liebrand TW, Cordewener JH, America AH, Xu X, Joosten MHAJ. 2014. Arabidopsis thaliana receptor-like protein AtRLP23 associates with the receptor-like kinase AtSOBIR1. Plant Signaling \& Behavior 9: e27937.

Białas A, Zess EK, De la Concepcion JC, Franceschetti M, Pennington HG, Yoshida K, Upson JL, Chanclud E, Wu C-H, Langner T, Maqbool A, Varden FA, Derevnina L, Belhaj K, Fujisaki K, Saitoh H, Terauchi R, Banfield MJ, Kamoun S. 2018. Lessons in Effector and NLR Biology of PlantMicrobe Systems. Molecular Plant-Microbe Interactions 31: 34-45.

Bigeard J, Colcombet J, Hirt H. 2015. Signaling mechanisms in pattern-triggered immunity (PTI). Molecular Plant 8: 521-539.

BirkenbihI RP, Liu S, Somssich IE. 2017. Transcriptional events defining plant immune responses. Current Opinion in Plant Biology 38: 1-9.

Bleckmann A, Weidtkamp-Peters S, Seidel CA, Simon R. 2010. Stem cell signaling in Arabidopsis requires CRN to localize CLV2 to the plasma membrane. Plant Physiology 152: 166-176.

Böhm H, Albert I, Fan L, Reinhard A, Nürnberger T. 2014a. Immune receptor complexes at the plant cell surface. Current Opinion in Plant Biology 20: 47-54.

Böhm H, Albert I, Oome S, Raaymakers TM, Van den Ackerveken G, Nürnberger T. 2014b. A conserved peptide pattern from a widespread microbial virulence factor triggers pattern-induced immunity in Arabidopsis. PLOS Pathogens 10: e1004491.

Boller T, Felix G. 2009. A renaissance of elicitors: perception of microbe-associated molecular patterns and danger signals by pattern-recognition receptors. Annual Review of Plant Biology 60: 379-406.

Bolton MD, Van Esse HP, Vossen JH, De Jonge R, Stergiopoulos I, Stulemeijer IJ, Van Den Berg G, Borrás-Hidalgo O, Dekker HL, De Koster CG. 2008. The novel Cladosporium fulvum lysin motif effector Ecp6 is a virulence factor with orthologues in other fungal species. Molecular Microbiology 69: 119-136.

Brutus A, Sicilia F, Macone A, Cervone F, De Lorenzo G. 2010. A domain swap approach reveals a role of the plant wall-associated kinase 1 (WAK1) as a receptor of oligogalacturonides. Proceedings of the National Academy of Sciences of the United States of America 107: 9452-9457.

Bücherl CA, Jarsch IK, Schudoma C, Segonzac C, Mbengue M, Robatzek S, MacLean D, Ott T, Zipfel C. 2017. Plant immune and growth receptors share common signalling components but localise to distinct plasma membrane nanodomains. eLife 6. e25114

Cao X. 2016. Self-regulation and cross-regulation of pattern-recognition receptor signalling in health and disease. Nature Reviews Immunology 16: 35-50.

Cao Y, Liang Y, Tanaka K, Nguyen CT, Jedrzejczak RP, Joachimiak A, Stacey G. 2014. The kinase LYK5 is a major chitin receptor in Arabidopsis and forms a chitin-induced complex with related kinase CERK1. elife 3: e03766. 
Catanzariti AM, Do HT, Bru P, Sain M, Thatcher LF, Rep M, Jones DA. 2017. The tomato I gene for Fusarium wilt resistance encodes an atypical leucine-rich repeat receptor-like protein whose function is nevertheless dependent on SOBIR1 and SERK3/BAK1. The Plant Journal 89: 1195-1209.

Cesari S. 2017. Multiple strategies for pathogen perception by plant immune receptors. New Phytologist. doi.org/10.1111/nph.14877

Chinchilla D, Shan L, He P, de Vries S, Kemmerling B. 2009. One for all: the receptor-associated kinase BAK1. Trends in Plant Science 14: 535-541.

Chinchilla D, Zipfel C, Robatzek S, Kemmerling B, Nürnberger T, Jones JD, Felix G, Boller T. 2007. A flagellin-induced complex of the receptor FLS2 and BAK1 initiates plant defence. Nature 448: 497500.

Choi J, Tanaka K, Cao Y, Qi Y, Qiu J, Liang Y, Lee SY, Stacey G. 2014. Identification of a plant receptor for extracellular ATP. Science 343: 290-294.

Clark SE, Williams RW, Meyerowitz EM. 1997. The CLAVATA1 gene encodes a putative receptor kinase that controls shoot and floral meristem size in Arabidopsis. Cell 89: 575-585.

Cook DE, Mesarich CH, Thomma BP. 2015. Understanding plant immunity as a surveillance system to detect invasion. Annual Review of Phytopathology 53: 541-563.

Couto D, Niebergall R, Liang X, Bücherl CA, Sklenar J, Macho AP, Ntoukakis V, Derbyshire P, Altenbach D, Maclean D, Robatzek S, Uhrig J, Menke F, Zhou J-M, Zipfel C. 2016. The Arabidopsis protein phosphatase PP2C38 negatively regulates the central immune kinase BIK1. PLOS Pathogens 12: e1005811.

Couto D, Zipfel C. 2016. Regulation of pattern recognition receptor signalling in plants. Nature Reviews Immunology 16: 537-552.

De Jong CF, Takken FL, Cai $X$, de Wit PJ, Joosten MHAJ. 2002. Attenuation of Cf-mediated defense responses at elevated temperatures correlates with a decrease in elicitor-binding sites. Molecular Plant-Microbe Interactions 15: 1040-1049.

De Jonge R, van Esse HP, Kombrink A, Shinya T, Desaki Y, Bours R, van der Krol S, Shibuya N, Joosten MHAJ, Thomma BP. 2010. Conserved fungal LysM effector Ecp6 prevents chitin-triggered immunity in plants. Science 329: 953-955.

De Smet I, Voß U, Jürgens G, Beeckman T. 2009. Receptor-like kinases shape the plant. Nature Cell Biology 11: 1166.

De Wit PJ. 1995. Fungal avirulence genes and plant resistance genes: unraveling the molecular basis of gene-for-gene interactions. Advances in Botanical Research 21: 147-185.

De Wit PJ. 1977. A light and scanning-electron microscopic study of infection of tomato plants by virulent and avirulent races of Cladosporium fulvum. European Journal of Plant Pathology 83: 109-122.

De Wit, P.J.G.M. 2016. Cladosporium fulvum effectors: weapons in the arms race with tomato. Annual Review of Phytopathology 54: 1-23

Deslandes L, Rivas S. 2012. Catch me if you can: bacterial effectors and plant targets. Trends in Plant Science 17: 644-655.

Dixon MS, Hatzixanthis K, Jones DA, Harrison K, Jones JD. 1998. The tomato Cf- 5 disease resistance gene and six homologs show pronounced allelic variation in leucine-rich repeat copy number. The Plant Cell 10: 1915-1925.

Dixon MS, Jones DA, Keddie JS, Thomas CM, Harrison K, Jones JD. 1996. The tomato Cf-2 disease resistance locus comprises two functional genes encoding leucine-rich repeat proteins. Cell 84: 451459.

Dodds PN, Rathjen JP. 2010. Plant immunity: towards an integrated view of plant-pathogen interactions. Nature Reviews Genetics 11: 539-548.

Domazakis E, Wouters D, Visser R, Kamoun S, Joosten MHAJ, Vleeshouwers VGAA. 2018. The ELR-SOBIR1 complex functions as a two-component RLK to mount defense against Phytophthora infestans. Molecular Plant-Microbe Interactions. 10.1094/mpmi-09-17-0217-r 
Domínguez-Ferreras A, Kiss-Papp M, Jehle A, Kristina, Felix G, Chinchilla D. 2015. An overdose of the Arabidopsis coreceptor BAK1 or its ectodomain causes autoimmunity in a SOBIR1-dependent manner. Plant Physiology 168: 1106-1121.

Dou D, Zhou J-M. 2012. Phytopathogen effectors subverting host immunity: different foes, similar battleground. Cell Host \& Microbe 12: 484-495.

Eckardt NA. 2011. BIK1 function in plant growth and defense signaling. The Plant Cell 23: 2806-2806

Etalo DW, Stulemeijer IJE, Peter van Esse H, de Vos RCH, Bouwmeester HJ, Joosten MHAJ. 2013. System-Wide hypersensitive response-associated transcriptome and metabolome reprogramming in tomato. Plant Physiology 162: 1599-1617.

Fenyk S, Townsend PD, Dixon CH, Spies GB, Campillo AdSE, Slootweg EJ, Westerhof LB, Gawehns FK, Knight MR, Sharples GJ. 2015. The potato nucleotide-binding leucine-rich repeat (NLR) immune receptor Rx1 is a pathogen-dependent DNA-deforming protein. Journal of Biological Chemistry 290: 24945-24960.

Fischer I, Diévart A, Droc G, Dufayard J-F, Chantret N. 2016. Evolutionary dynamics of the Leucine-Rich Repeats Receptor-Like Kinase (LRR-RLK) subfamily in angiosperms. Plant Physiology 170: 1595-1610.

Frescatada-Rosa M, Robatzek S, Kuhn H. 2015. Should I stay or should I go? Traffic control for plant pattern recognition receptors. Current Opinion in Plant Biology 28: 23-29.

Fritz-Laylin LK, Krishnamurthy N, Tör M, Sjölander KV, Jones JD. 2005. Phylogenomic analysis of the receptor-like proteins of rice and Arabidopsis. Plant Physiology 138: 611-623.

Gabriëls SH, Vossen JH, Ekengren SK, Ooijen Gv, Abd-El-Haliem AM, Berg G, Rainey DY, Martin GB, Takken FL, de Wit PJ. 2007. An NB-LRR protein required for HR signalling mediated by both extraand intracellular resistance proteins. The Plant Journal 50: 14-28.

Gao M, Wang X, Wang D, Xu F, Ding X, Zhang Z, Bi D, Cheng YT, Chen S, Li X, Zhang Y. 2009. Regulation of cell death and innate immunity by two receptor-like kinases in Arabidopsis. Cell Host and Microbe 6: 34-44.

Geisler M, Nadeau J, Sack FD. 2000. Oriented asymmetric divisions that generate the stomatal spacing pattern in Arabidopsis are disrupted by the too many mouths mutation. The Plant Cell 12: 2075-2086.

Gish LA, Clark SE. 2011. The RLK/Pelle family of kinases. The Plant Journal 66: 117-127.

Gómez-Gómez L, Boller T. 2000. FLS2: an LRR receptor-like kinase involved in the perception of the bacterial elicitor flagellin in Arabidopsis. Molecular Cell 5: 1003-1011.

Goodin MM, Zaitlin D, Naidu RA, Lommel SA. 2008. Nicotiana benthamiana: its history and future as a model for plant-pathogen interactions. Molecular Plant-Microbe Interactions 21: 1015-1026.

Groner WD, Christy ME, Kreiner CM, Liljegren SJ. 2016. Allele-Specific Interactions between CAST AWAY and NEVERSHED Control Abscission in Arabidopsis Flowers. Frontiers in Plant Science 7: 1588.

Gubert CM, Liljegren SJ. 2014. HAESA and HAESA-LIKE2 activate organ abscission downstream of NEVERSHED and EVERSHED in Arabidopsis flowers. Plant Signaling \& Behavior 9: e29115.

Gust AA, Felix G. 2014. Receptor like proteins associate with SOBIR1-type of adaptors to form bimolecular receptor kinases. Current Opinion in Plant Biology 21: 104-111.

Gust AA, Pruitt R, Nürnberger T. 2017. Sensing Danger: Key to Activating Plant Immunity. Trends in Plant Science 22: 779-791.

Gust AA, Willmann R, Desaki Y, Grabherr HM, Nürnberger T. 2012. Plant LysM proteins: modules mediating symbiosis and immunity. Trends in Plant Science 17: 495-502.

Halter T, Imkampe J, Blaum BS, Stehle T, Kemmerling B. 2014a. BIR2 affects complex formation of BAK1 with ligand binding receptors in plant defense. Plant Signaling \& Behavior 9: 134-143.

Halter T, Imkampe J, Mazzotta S, Wierzba M, Postel S, Bücherl C, Kiefer C, Stahl M, Chinchilla D, Wang X, Nürnberger T, Zipfel C, Clouse S, Borst Jan W, Boeren S, de Vries Sacco C, Tax F, Kemmerling B. 2014b. The Leucine-Rich Repeat Receptor Kinase BIR2 Is a Negative Regulator of BAK1 in Plant Immunity. Current Biology 24: 134-143.

Hao H, Fan L, Chen T, Li R, Li X, He Q, Botella MA, Lin J. 2014. Clathrin and membrane microdomains cooperatively regulate RbohD dynamics and activity in Arabidopsis. The Plant Cell 26: 1729-1745. 
Heese A, Hann DR, Gimenez-Ibanez S, Jones AME, He K, Li J, Schroeder JI, Peck SC, Rathjen JP. 2007. The receptor-like kinase SERK3/BAK1 is a central regulator of innate immunity in plants. Proceedings of the National Academy of Sciences of the United States of America 104: 12217-12222.

Hegenauer V, Fürst U, Kaiser B, Smoker M, Zipfel C, Felix G, Stahl M, Albert M. 2016. Detection of the plant parasite Cuscuta reflexa by a tomato cell surface receptor. Science 353: 478-481.

Hohmann U, Lau K, Hothorn M. 2017. The structural basis of ligand perception and signal activation by receptor kinases. Annual Review of Plant Biology 68: 109-137.

Hohmann U, Santiago J, Nicolet J, Olsson V, Spiga FM, Hothorn LA, Butenko MA, Hothorn M. 2018. Mechanistic basis for the activation of plant membrane receptor kinases by SERK-family coreceptors. Proceedings of the National Academy of Sciences of the United States of America. 10.1073/pnas. 1714972115

Hückelhoven R. 2007. Cell wall-associated mechanisms of disease resistance and susceptibility. Annual Review of Phytopathology 45: 101-127.

Hutten SJ, Hamers DS, Den Toorn MA, Van Esse W, Nolles A, Bücherl CA, De Vries SC, Hohlbein J, Borst JW. 2017. Visualization of BRI1 and SERK3/BAK1 Nanoclusters in Arabidopsis Roots. PLoS ONE 12: e0169905.

Jacob F, Vernaldi S, Maekawa T. 2013. Evolution and conservation of plant NLR functions. Frontiers in Immunology 4: 297.

Jarsch IK, Konrad SSA, Stratil TF, Urbanus SL, Szymanski W, Braun P, Braun K-H, Ott T. 2014. Plasma membranes are subcompartmentalized into a plethora of coexisting and diverse microdomains in Arabidopsis and Nicotiana benthamiana. The Plant Cell 26. 1698-1711

Jehle AK, Fürst $\mathbf{U}$, Lipschis $\mathbf{M}$, Albert M, Felix G. 2013. Perception of the novel MAMP eMax from different Xanthomonas species requires the Arabidopsis receptor-like protein ReMAX and the receptor kinase SOBIR. Plant Signaling \& Behavior 8: e27408.

Jeong S, Trotochaud AE, Clark SE. 1999. The Arabidopsis CLAVATA2 gene encodes a receptor-like protein required for the stability of the CLAVATA1 receptor-like kinase. The Plant Cell 11: 1925-1933.

Jones DA, Thomas CM, Hammond-Kosack KE, Balint-Kurti PJ, Jones JD. 1994. Isolation of the tomato Cf-9 gene for resistance to Cladosporium fulvum by transposon tagging. Science 266: 789-793.

Jones JD, Dangl JL. 2006. The plant immune system. Nature 444: 323-329.

Jones JD, Vance RE, Dangl JL. 2016. Intracellular innate immune surveillance devices in plants and animals. Science 354: aaf6395.

Joosten MHAJ, De Wit P. 1999. The tomato-Cladosporium fulvum interaction: A versatile experimental system to study plant-pathogen interactions. Annual Review of Phytopathology 37: 335-367.

Joosten MHAJ, Cozijnsen TJ, De Wit PJ. 1994. Host resistance to a fungal tomato pathogen lost by a single base-pair change in an avirulence gene. Nature 367: 384.

Joosten MHAJ, Vogelsang R, Cozijnsen TJ, Verberne MC, De Wit PJ. 1997. The biotrophic fungus Cladosporium fulvum circumvents Cf-4-mediated resistance by producing unstable AVR4 elicitors. The Plant Cell 9: 367-379.

Jordá L, Sopeña-Torres S, Escudero V, Nuñez-Corcuera B, Delgado-Cerezo M, Torii KU, Molina A. 2016. ERECTA and BAK1 receptor like kinases interact to regulate immune responses in Arabidopsis. Frontiers in Plant Science 7: 897.

Kadota Y, Shirasu K, Zipfel C. 2015. Regulation of the NADPH oxidase RBOHD during plant immunity. Plant and Cell Physiology 56: 1472-1480.

Kadota Y, Sklenar J, Derbyshire P, Stransfeld L, Asai S, Ntoukakis V, Jones Jonathan D, Shirasu K, Menke F, Jones A, Zipfel C. 2014. Direct regulation of the NADPH Oxidase RBOHD by the PRRAssociated Kinase BIK1 during plant immunity. Molecular Cell 54: 43-55

Khan M, Seto D, Subramaniam R, Desveaux D. 2018. Oh, the places they'll go! A survey of phytopathogen effectors and their host targets. The Plant Journal 93: 651-663.

Kohler AC, Chen L-H, Hurlburt N, Salvucci A, Schwessinger B, Fisher AJ, Stergiopoulos I. 2016. Structural analysis of an Avr4 effector ortholog offers insight into chitin-binding and recognition by the Cf-4 receptor. The Plant Cell 28: 1945-1965 
Kohorn BD, Kohorn SL. 2012. The cell wall-associated kinases, WAKs, as pectin receptors. Frontiers in Plant Science 3: 88.

Kourelis J, van der Hoorn RAL. 2018. Defended to the nines: 25 years of resistance gene cloning identifies nine mechanisms for R protein function. The Plant Cell 30: 285-299.

Lal NK, Nagalakshmi U, Hurlburt NK, Flores R, Bak A, Sone P, Ma X, Song G, Walley J, Shan L, He P, Casteel C, Fisher AJ, Dinesh-Kumar SP. 2018. The receptor-like cytoplasmic kinase BIK1 localizes to the nucleus and regulates defense hormone expression during plant innate immunity. Cell Host \& Microbe 23: 485-497.

Laurent F, Labesse G, de Wit P. 2000. Molecular cloning and partial characterization of a plant VAP33 homologue with a major sperm protein domain. Biochemical and Biophysical Research Communications 270: 286-292.

Lee J, Eschen-Lippold L, Lassowskat I, Böttcher C, Scheel D. 2015. Cellular reprogramming through mitogen-activated protein kinases. Frontiers in Plant Science 6: 940.

Lee JS, Kuroha T, Hnilova M, Khatayevich D, Kanaoka MM, McAbee JM, Sarikaya M, Tamerler C, Torii KU. 2012. Direct interaction of ligand-receptor pairs specifying stomatal patterning. Genes \& Development 26: 126-136.

Lehti-Shiu MD, Zou C, Hanada K, Shiu S-H. 2009. Evolutionary history and stress regulation of plant receptor-like kinase/pelle genes. Plant Physiology 150: 12-26.

Leslie ME, Lewis MW, Youn JY, Daniels MJ, Liljegren SJ. 2010. The EVERSHED receptor-like kinase modulates floral organ shedding in Arabidopsis. Development 137: 467-476.

Li B, Meng X, Shan L, He P. 2016a. Transcriptional regulation of pattern-triggered immunity in plants. Cell Host \& Microbe 19: 641-650.

Li J, Chory J. 1997. A putative leucine-rich repeat receptor kinase involved in brassinosteroid signal transduction. Cell 90: 929-938.

Li J, Tax FE. 2013. Receptor-like kinases: key regulators of plant development and defense. Journal of Integrative Plant Biology 55: 1184-1187.

Li J, Wen J, Lease KA, Doke JT, Tax FE, Walker JC. 2002. BAK1, an Arabidopsis LRR receptor-like protein kinase, interacts with BRI1 and modulates brassinosteroid signaling. Cell 110: 213-222.

Li J, Zhao-Hui C, Batoux M, Nekrasov V, Roux M, Chinchilla D, Zipfel C, Jones JD. 2009. Specific ER quality control components required for biogenesis of the plant innate immune receptor EFR. Proceedings of the National Academy of Sciences of the United States of America 106: 15973-15978.

Li L, Li M, Yu L, Zhou Z, Liang X, Liu Z, Cai G, Gao L, Zhang X, Wang Y, Chen S, Zhou J-M. 2014. The FLS2-associated ainase BIK1 directly phosphorylates the NADPH oxidase RbohD to control plant immunity. Cell Host \& Microbe 15: 329-338.

Li L, Yu Y, Zhou Z, Zhou J-M. 2016b. Plant pattern-recognition receptors controlling innate immunity. Science China Life Sciences 59: 878-888.

Liang X, Ding P, Lian K, Wang J, Ma M, Li L, Li L, Li M, Zhang X, Chen S. 2016. Arabidopsis heterotrimeric $\mathrm{G}$ proteins regulate immunity by directly coupling to the FLS2 receptor. eLife 5: e13568.

Liang X, Zhou J-M. 2018. Receptor-like cytoplasmic kinases: central players in plant receptor kinasemediated signaling. Annual Review of Plant Biology 69: 267-299.

Liebrand TWH, Smit P, Abd-El-Haliem A, de Jonge R, Cordewener JHG, America AHP, Sklenar J, Jones AME, Robatzek S, Thomma BPHJ, Tameling WIL, Joosten MHAJ. 2012. Endoplasmic reticulum-quality control chaperones facilitate the biogenesis of $\mathrm{Cf}$ receptor-like proteins involved in pathogen resistance of tomato. Plant Physiology 159: 1819-1833.

Liebrand TWH, van den Berg GCM, Zhang Z, Smit P, Cordewener JHG, America AHP, Sklenar J, Jones AME, Tameling WIL, Robatzek S, Thomma BPHJ, Joosten MHAJ. 2013. Receptor-like kinase SOBIR1/EVR interacts with receptor-like proteins in plant immunity against fungal infection. Proceedings of the National Academy of Sciences of the United States of America 110: 10010-10015.

Liebrand TWH, van den Burg HA, Joosten MHAJ. 2014. Two for all: receptor-associated kinases SOBIR1 and BAK1. Trends in Plant Science 19: 123-132. 
Liljegren SJ. 2012. Organ abscission: exit strategies require signals and moving traffic. Current Opinion in Plant Biology 15: 670-676.

Liljegren SJ, Leslie ME, Darnielle L, Lewis MW, Taylor SM, Luo R, Geldner N, Chory J, Randazzo PA, Yanofsky MF, Ecker JR. 2009. Regulation of membrane trafficking and organ separation by the NEVERSHED ARF-GAP protein. Development 136: 1909-1918.

Lin W, Lu D, Gao X, Jiang S, Ma X, Wang Z, Mengiste T, He P, Shan L. 2013. Inverse modulation of plant immune and brassinosteroid signaling pathways by the receptor-like cytoplasmic kinase BIK1. Proceedings of the National Academy of Sciences of the United States of America 110: 12114-12119.

Liu Y, Huang X, Li M, He P, Zhang YC. 2016. Loss-of-function of Arabidopsis receptor-like kinase BIR1 activates cell death and defense responses mediated by BAK1 and SOBIR1. New Phytologist 212: 637-645.

Lorrain S, Vailleau F, Balagué C, Roby D. 2003. Lesion mimic mutants: keys for deciphering cell death and defense pathways in plants? Trends in Plant Science 8: 263-271.

Lozano-Durán R, Zipfel C. 2015. Trade-off between growth and immunity: role of brassinosteroids. Trends in Plant Science 20: 12-19.

Lu D, Wu S, Gao X, Zhang Y, Shan L, He P. 2010. A receptor-like cytoplasmic kinase, BIK1, associates with a flagellin receptor complex to initiate plant innate immunity. Proceedings of the National Academy of Sciences of the United States of America 107: 496-501.

Luderer R, Takken FL, de Wit PJ, Joosten MHAJ. 2002. Cladosporium fulvum overcomes Cf-2-mediated resistance by producing truncated AVR2 elicitor proteins. Molecular Microbiology 45: 875-884.

Ma L, Borhan MH. 2015. The receptor-like kinase SOBIR1 interacts with Brassica napus LepR3 and is required for Leptosphaeria maculans AvrLm1-triggered immunity. Frontiers in Plant Science 6: 933.

Ma X, Xu G, He P, Shan L. 2016. SERKing Coreceptors for Receptors. Trends in Plant Science 21: 10171033.

Macho AP, Lozano-Durán R, Zipfel C. 2015. Importance of tyrosine phosphorylation in receptor kinase complexes. Trends in Plant Science 20: 269-272.

Macho AP, Zipfel C. 2014. Plant PRRs and the activation of innate immune signaling. Molecular Cell 54: 263-272.

Martin J. 1964. Role of cuticle in the defense against plant disease. Annual Review of Phytopathology 2: 81-100.

May MJ, Hammond-Kosack KE, Jones JD. 1996. Involvement of reactive oxygen species, glutathione metabolism, and lipid peroxidation in the Cf-gene-dependent defense response of tomato cotyledons induced by race-specific elicitors of Cladosporium fulvum. Plant Physiology 110: 13671379 .

Mbengue M, Bourdais G, Gervasi F, Beck M, Zhou J, Spallek T, Bartels S, Boller T, Ueda T, Kuhn H, Robatzek S. 2016. Clathrin-dependent endocytosis is required for immunity mediated by pattern recognition receptor kinases. Proceedings of the National Academy of Sciences of the United States of America 113: 11034-11039.

Mesarich CH, Griffiths SA, van der Burgt A, Ökmen B, Beenen HG, Etalo DW, Joosten MHAJ, de Wit PJ. 2014. Transcriptome sequencing uncovers the Avr5 avirulence gene of the tomato leaf mold pathogen Cladosporium fulvum. Molecular Plant-Microbe Interactions 27: 846-857.

Mesarich CH, Stergiopoulos I, Beenen HG, Cordovez V, Guo Y, Karimi Jashni M, Bradshaw RE, de Wit PJGM. 2015. A conserved proline residue in Dothideomycete Avr4 effector proteins is required to trigger a Cf-4-dependent hypersensitive response. Molecular Plant Pathology 17: 84-95.

Müller R, Bleckmann A, Simon R. 2008. The receptor kinase CORYNE of Arabidopsis transmits the stem cell-limiting signal CLAVATA3 independently of CLAVATA1. The Plant Cell 20: 934-946.

Nadeau JA, Sack FD. 2002. Control of stomatal distribution on the Arabidopsis leaf surface. Science 296: 1697-1700.

Nam KH, Li J. 2002. BRI1/BAK1, a receptor kinase pair mediating brassinosteroid signaling. Cell 110: 203-212. 
Nekrasov V, Ludwig AA, Jones JD. 2006. CITRX thioredoxin is a putative adaptor protein connecting $\mathrm{Cf}-9$ and the ACIK1 protein kinase during the Cf-9/Avr9-induced defence response. FEBS letters 580: 4236-4241.

Niederhuth CE, Cho SK, Seitz K, Walker JC. 2013. Letting go is never easy: Abscission and receptor-like protein kinases. Journal of Integrative Plant Biology 55: 1251-1263.

Ogawa M, Shinohara H, Sakagami Y, Matsubayashi Y. 2008. Arabidopsis CLV3 peptide directly binds CLV1 ectodomain. Science 319: 294.

Pan L, Lv S, Yang N, Lv Y, Liu Z, Wu J, Wang G. 2016. The Multifunction of CLAVATA2 in Plant Development and Immunity. Frontiers in Plant Science 7: 1573.

Park C-J, Peng Y, Chen X, Dardick C, Ruan D, Bart R, Canlas PE, Ronald PC. 2008. Rice XB15, a protein phosphatase $2 \mathrm{C}$, negatively regulates cell death and XA21-mediated innate immunity. PLoS Biology 6: e231.

Parniske M, Hammond-Kosack KE, Golstein C, Thomas CM, Jones DA, Harrison K, Wulff BB, Jones JD. 1997. Novel disease resistance specificities result from sequence exchange between tandemly repeated genes at the Cf-4/9 locus of tomato. Cell 91: 821-832.

Peng K-C, Wang C-W, Wu C-H, Huang C-T, Liou R-F. 2015. Tomato SOBIR1/EVR homologs are involved in elicitin perception and plant defense against the oomycete pathogen Phytophthora parasitica. Molecular Plant-Microbe Interactions 28: 913-926.

Piedras P, Hammond-Kosack KE, Harrison K, Jones JD. 1998. Rapid, Cf-9-and Avr9-dependent production of active oxygen species in tobacco suspension cultures. Molecular Plant-Microbe Interactions 11: 1155-1166.

Piedras P, Rivas S, Dröge S, Hillmer S, Jones JDG. 2000. Functional, c-myc-tagged Cf-9 resistance gene products are plasma-membrane localized and glycosylated. The Plant Journal 21: 529-536.

Pieterse CM, Van der Does D, Zamioudis C, Leon-Reyes A, Van Wees SC. 2012. Hormonal modulation of plant immunity. Annual Review of Cell and Developmental Biology 28: 489-521.

Postma J, Liebrand TWH, Bi G, Evrard A, Bye RR, Mbengue M, Kuhn H, Joosten MHAJ, Robatzek SC. 2016. Avr4 promotes $\mathrm{Cf}-4$ receptor-like protein association with the BAK1/SERK3 receptor-like kinase to initiate receptor endocytosis and plant immunity. New Phytologist 210: 627-642.

Ranf S, Gisch N, Schäffer M, Illig T, Westphal L, Knirel YA, Sánchez-Carballo PM, Zähringer U, Hückelhoven R, Lee J. 2015. A lectin S-domain receptor kinase mediates lipopolysaccharide sensing in Arabidopsis thaliana. Nature Immunology 16: 426-433.

Rivas S, Rougon-Cardoso A, Smoker M, Schauser L, Yoshioka H, Jones JD. 2004. CITRX thioredoxin interacts with the tomato $\mathrm{Cf}-9$ resistance protein and negatively regulates defence. The EMBO Journal 23: 2156-2165.

Rivas S, Thomas CM. 2005. Molecular interactions between tomato and the leaf mold pathogen Cladosporium fulvum. Annual Review Phytopathology 43: 395-436.

Romeis T, Ludwig AA, Martin R, Jones JD. 2001. Calcium-dependent protein kinases play an essential role in a plant defence response. The EMBO Journal 20: 5556-5567.

Romeis T, Piedras P, Jones JD. 2000. Resistance gene-dependent activation of a calcium-dependent protein kinase in the plant defense response. The Plant Cell 12: 803-815.

Romeis T, Piedras P, Zhang S, Klessig DF, Hirt H, Jones JD. 1999. Rapid Avr9-and Cf-9-dependent activation of MAP kinases in tobacco cell cultures and leaves: Convergence of resistance gene, elicitor, wound, and salicylate responses. The Plant Cell 11: 273-287.

Rooney HC, van't Klooster JW, van der Hoorn RA, Joosten MHAJ, Jones JD, de Wit PJ. 2005. Cladosporium Avr2 inhibits tomato Rcr3 protease required for Cf-2-dependent disease resistance. Science 308: 1783-1786.

Rowland O, Ludwig AA, Merrick CJ, Baillieul F, Tracy FE, Durrant WE, Fritz-Laylin L, Nekrasov V, Sjölander K, Yoshioka H. 2005. Functional analysis of Avr9/Cf-9 rapidly elicited genes identifies a protein kinase, $\mathrm{ACIK1}$, that is essential for full $\mathrm{Cf}-9$-dependent disease resistance in tomato. The Plant Cell 17: 295-310. 
Sakamoto T, Deguchi M, Brustolini OJB, Santos AA, Silva FF, Fontes EPB. 2012. The tomato RLK superfamily: phylogeny and functional predictions about the role of the LRRII-RLK subfamily in antiviral defense. BMC Plant Biology 12: 229.

Sandrock RW, van Etten HD. 1998. Fungal sensitivity to and enzymatic degradation of the phytoanticipin $\alpha$-tomatine. Phytopathology 88: 137-143.

Saur IM, Kadota Y, Sklenar J, Holton NJ, Smakowska E, Belkhadir Y, Zipfel C, Rathjen JP. 2016. $\mathrm{NbCSPR}$ underlies age-dependent immune responses to bacterial cold shock protein in Nicotiana benthamiana. Proceedings of the National Academy of Sciences of the United States of America 113: 3389-3394.

Schornack S, Huitema E, Cano LM, Bozkurt TO, Oliva R, van Damme M, Schwizer S, Raffaele S, CHAPARRO-GARCIA A, Farrer R. 2009. Ten things to know about oomycete effectors. Molecular Plant Pathology 10: 795-803.

Schulze B, Mentzel T, Jehle AK, Mueller K, Beeler S, Boller T, Felix G, Chinchilla D. 2010. Rapid heteromerization and phosphorylation of ligand-activated plant transmembrane receptors and their associated kinase BAK1. Journal of Biological Chemistry 285: 9444-9451.

Segonzac C, Macho AP, Sanmartín M, Ntoukakis V, Sánchez-Serrano JJ, Zipfel C. 2014. Negative control of BAK1 by protein phosphatase $2 \mathrm{~A}$ during plant innate immunity. The EMBO Journal 33 : 2069-2079.

Seybold H, Trempel F, Ranf S, Scheel D, Romeis T, Lee J. 2014. Ca2+ signalling in plant immune response: from pattern recognition receptors to $\mathrm{Ca} 2+$ decoding mechanisms. New Phytologist 204: 782-790.

Shaw AS, Kornev AP, Hu J, Ahuja LG, Taylor SS. 2014. Kinases and pseudokinases: lessons from RAF. Molecular and Cellular Biology 34: 1538-1546.

Shpak ED, McAbee JM, Pillitteri LJ, Torii KU. 2005. Stomatal patterning and differentiation by synergistic interactions of receptor kinases. Science 309: 290-293.

Singh P, Zimmerli L. 2013. Lectin receptor kinases in plant innate immunity. Frontiers in Plant Science 4: 124.

Smakowska E, Kong J, Busch W, Belkhadir Y. 2016. Organ-specific regulation of growth-defense tradeoffs by plants. Current Opinion in Plant Biology 29: 129-137.

Somssich M, Ma Q, Weidtkamp-Peters S, Stahl Y, Felekyan S, Bleckmann A, Seidel CA, Simon R. 2015. Real-time dynamics of peptide ligand-dependent receptor complex formation in planta. Science Signaling 8: 76.

Stam R, ScheikI D, Tellier A. 2017. The wild tomato species Solanum chilense shows variation in pathogen resistance between geographically distinct populations. PeerJ 5: e2910.

Stergiopoulos I, van den Burg HA, Ökmen B, Beenen HG, van Liere S, Kema GH, de Wit PJ. 2010. Tomato $\mathrm{Cf}$ resistance proteins mediate recognition of cognate homologous effectors from fungi pathogenic on dicots and monocots. Proceedings of the National Academy of Sciences of the United States of America 107: 7610-7615.

Stulemeijer IJE. 2008. Phosphorylation and proteome dynamics in pathogen-resistant tomato plants. $\mathrm{PhD}$ thesis, Wageningen University. Chapter 6: p175-189

Stulemeijer IJE, Stratmann JW, Joosten MHAJ. 2007. Tomato mitogen-activated protein kinases LeMPK1, LeMPK2, and LeMPK3 are activated during the Cf-4/Avr4-induced hypersensitive response and have distinct phosphorylation specificities. Plant Physiology 144: 1481-1494.

Sun Y, Li L, Macho AP, Han Z, Hu Z, Zipfel C, Zhou JM, Chai J. 2013. Structural basis for flg22-induced activation of the Arabidopsis FLS2-BAK1 immune complex. Science 342: 624-628.

Takken FL, Goverse A. 2012. How to build a pathogen detector: structural basis of NB-LRR function. Current Opinion in Plant Biology 15: 375-384.

Takken FL, Thomas CM, Joosten MHAJ, Golstein C, Westerink N, Hille J, Nijkamp HJJ, De Wit PJ, Jones JD. 1999. A second gene at the tomato Cf-4 locus confers resistance to Cladosporium fulvum through recognition of a novel avirulence determinant. The Plant Journal 20: 279-288. 
Tang D, Wang G, Zhou J-M. 2017. Receptor kinases in plant-pathogen interactions: more than pattern recognition. The Plant Cell 29: 618-637.

Tang W, Kim T-W, Oses-Prieto JA, Sun Y, Deng Z, Zhu S, Wang R, Burlingame AL, Wang Z-Y. 2008. BSKs mediate signal transduction from the receptor kinase BRI1 in Arabidopsis. Science 321: 557-560.

Thomas CM, Jones DA, Parniske M, Harrison K, Balint-Kurti PJ, Hatzixanthis K, Jones JD. 1997. Characterization of the tomato $\mathrm{Cf}-4$ gene for resistance to Cladosporium fulvum identifies sequences that determine recognitional specificity in Cf-4 and Cf-9. The Plant Cell 9: 2209-2224.

Thomas CM, Mark S. Dixon, Martin Parniske, Catherine Golstein and Jonathan D. G. Jones. 1998. Genetic and molecular analysis of tomato Cf genes for resistance to Cladosporium fulvum. Philosophical Transactions of the Royal Society B: Biological Sciences 353: 1413-1424.

Thomma BPHJ, Nürnberger T, Joosten MHAJ. 2011. Of PAMPs and effectors: The blurred PTI-ETI dichotomy. The Plant Cell 23: 4-15.

Thomma BPHJ, Van Esse HP, Crous PW, De Wit PJGM. 2005. Cladosporium fulvum (syn. Passalora fulva), a highly specialized plant pathogen as a model for functional studies on plant pathogenic Mycosphaerellaceae. Molecular Plant Pathology 6: 379-393.

Van den Ackerveken GF, van Kan JA, de Wit PJ. 1992. Molecular analysis of the avirulence gene avr9 of the fungal tomato pathogen Cladosporium fulvum fully supports the gene-for-gene hypothesis. The Plant Journal 2: 359-366.

Van den Burg HA, De Wit PJ, Vervoort J. 2001. Efficient $13 \mathrm{C} / 15 \mathrm{~N}$ double labeling of the avirulence protein AVR4 in a methanol-utilizing strain (Mut+) of Pichia pastoris. Journal of Biomolecular NMR 20: 251-261.

Van den Burg HA, Harrison SJ, Joosten MHAJ, Vervoort J, De Wit PJGM. 2006. Cladosporium fulvum Avr4 protects fungal cell walls against hydrolysis by plant chitinases accumulating during infection. Molecular Plant-Microbe Interactions 19: 1420-1430.

Van den Burg HA, Westerink N, Francoijs K-J, Roth R, Woestenenk E, Boeren S, de Wit PJ, Joosten MHAJ, Vervoort J. 2003. Natural disulfide bond-disrupted mutants of AVR4 of the tomato pathogen Cladosporium fulvum are sensitive to proteolysis, circumvent Cf-4-mediated resistance, but retain their chitin binding ability. Journal of Biological Chemistry 278: 27340-27346.

Van der Hoorn RA, De Wit PJ, Joosten MHAJ. 2002. Balancing selection favors guarding resistance proteins. Trends in Plant Science 7: 67-71.

Van der Hoorn RAL, Laurent F, Roth R, de Wit PJGM. 2000. Agroinfiltration is a versatile tool that facilitates comparative analyses of Avr9/Cf-9-induced and Avr4/Cf-4-induced necrosis. Molecular Plant-Microbe Interactions 13: 439-446.

Van Esse HP, Bolton MD, Stergiopoulos I, de Wit PJ, Thomma BP. 2007. The chitin-binding Cladosporium fulvum effector protein Avr4 is a virulence factor. Molecular Plant-Microbe Interactions 20: 10921101.

Van Esse HP, van't Klooster JW, Bolton MD, Yadeta KA, Van Baarlen P, Boeren S, Vervoort J, de Wit PJ, Thomma BP. 2008. The Cladosporium fulvum virulence protein Avr2 inhibits host proteases required for basal defense. The Plant Cell 20: 1948-1963.

Veronese P, Nakagami H, Bluhm B, AbuQamar S, Chen X, Salmeron J, Dietrich RA, Hirt H, Mengiste T. 2006. The membrane-anchored BOTRYTIS-INDUCED KINASE1 plays distinct roles in Arabidopsis resistance to necrotrophic and biotrophic pathogens. The Plant Cell 18: 257-273.

Wang G, Ellendorff U, Kemp B, Mansfield JW, Forsyth A, Mitchell K, Bastas K, Liu C-M, Woods-Tör A, Zipfel C. 2008. A genome-wide functional investigation into the roles of receptor-like proteins in Arabidopsis. Plant Physiology 147: 503-517.

Wang G, Fiers M, Ellendorff U, Wang Z, de Wit PJ, Angenent GC, Thomma BP. 2010. The diverse roles of extracellular leucine-rich repeat-containing receptor-like proteins in plants. Critical Reviews in Plant Science 29: 285-299.

Wang J, Grubb LE, Wang J, Liang X, Li L, Gao C, Ma M, Feng F, Li M, Li L, Zhang X, Yu F, Xie Q, Chen S, Zipfel C, Monaghan J, Zhou J-M. 2018a. A regulatory module controlling homeostasis of a plant immune kinase. Molecular Cell 69: 493-504. 
Wang L, Albert M, Einig E, Fürst U, Krust D, Felix G. 2016. The pattern-recognition receptor CORE of Solanaceae detects bacterial cold-shock protein. Nature Plants 2: 16185.

Wang L, Li H, Lv X, Chen T, Li R, Xue Y, Jiang J, Jin B, Baluška F, Šamaj J. 2015. Spatiotemporal dynamics of the BRI1 receptor and its regulation by membrane microdomains in living Arabidopsis cells. Molecular Plant 8: 1334-1349.

Wang Y, Bouwmeester K. 2017. L-type lectin receptor kinases: New forces in plant immunity. PLOS Pathogens 13: e1006433.

Wang Y, Xu Y, Sun Y, Wang H, Qi J, Wan B, Ye W, Lin Y, Shao Y, Dong S, Tyler BM, Wang Y. 2018b. Leucine-rich repeat receptor-like gene screen reveals that Nicotiana RXEG1 regulates glycoside hydrolase 12 MAMP detection. Nature Communications 9: 594.

Westerink N, Brandwagt BF, De Wit PJ, Joosten MHAJ. 2004. Cladosporium fulvum circumvents the second functional resistance gene homologue at the $\mathrm{Cf}-4$ locus ( $\mathrm{Hcr} 9-4 \mathrm{E})$ by secretion of a stable avr4E isoform. Molecular Microbiology 54: 533-545.

Win J, Chaparro-Garcia A, Belhaj K, Saunders D, Yoshida K, Dong S, Schornack S, Zipfel C, Robatzek S, Hogenhout S. 2012. Effector biology of plant-associated organisms: concepts and perspectives. Cold Spring Harbor symposia on quantitative biology. Cold Spring Harbor Laboratory Press, p. 235247.

Wu C-H, Abd-El-Haliem A, Bozkurt TO, Belhaj K, Terauchi R, Vossen JH, Kamoun S. 2017. NLR network mediates immunity to diverse plant pathogens. Proceedings of the National Academy of Sciences of the United States of America 114: 8113-8118.

Yan H, Zhao Y, Shi H, Li J, Wang Y, Tang D. 2018. BRASSINOSTEROID-SIGNALING KINASE1 phosphorylates MAPKKK5 to regulate immunity in Arabidopsis. Plant Physiology 176: 2991-3002

Yasuda S, Okada K, Saijo Y. 2017. A look at plant immunity through the window of the multitasking coreceptor BAK1. Current Opinion in Plant Biology 38: 10-18.

Zhang J, Li W, Xiang T, Liu Z, Laluk K, Ding X, Zou Y, Gao M, Zhang X, Chen S, Mengiste T, Zhang Y, Zhou JM. 2010. Receptor-like cytoplasmic kinases integrate signaling from multiple plant immune receptors and are targeted by a Pseudomonas syringae effector. Cell Host \& Microbe 7: 290-301.

Zhang L, Kars I, Essenstam B, Liebrand TW, Wagemakers L, Elberse J, Tagkalaki P, Tjoitang D, van den Ackerveken G, van Kan JA. 2014. Fungal endopolygalacturonases are recognized as microbeassociated molecular patterns by the Arabidopsis receptor-like protein RESPONSIVENESS TO BOTRYTIS POLYGALACTURONASES1. Plant Physiology 164: 352-364.

Zhang M, Su J, Zhang Y, Xu J, Zhang S. 2018. Conveying endogenous and exogenous signals: MAPK cascades in plant growth and defense. Current Opinion in Plant Biology 45, Part A: 1-10.

Zhang W, Fraiture M, Kolb D, Löffelhardt B, Desaki Y, Boutrot FFG, Tör M, Zipfel C, Gust AA, Brunner F. 2013. Arabidopsis receptor-like protein 30 and receptor-like kinase Suppressor Of BIR11/EVERSHED mediate innate immunity to necrotrophic fungi. The Plant Cell 25: 4227-4241.

Zhang X, Dodds PN, Bernoux M. 2017. What do we know about NOD-like receptors in plant immunity? Annual Review of Phytopathology 55: 205-229.

Zipfel C. 2014. Plant pattern-recognition receptors. Trends in Immunology 35: 345-351.

Zipfel C, Oldroyd GED. 2017. Plant signalling in symbiosis and immunity. Nature 543: 328-336. 


\section{Chapter 2}

\section{The bacterial effector AvrPto targets the regulatory co-receptor SOBIR1 and suppresses defence signalling mediated by the receptor-like protein Cf-4}

Jinbin $\mathrm{Wu}^{1}$, Aranka M. van der Burgh ${ }^{1}$, Guozhi $\mathrm{Bi}^{1+}$, Lisha Zhang ${ }^{1 \neq}$, James R. Alfano ${ }^{2,3}$, Gregory B. Martin ${ }^{4,5}$, and Matthieu H. A. J. Joosten ${ }^{1}$

${ }^{1}$ Laboratory of Phytopathology, Wageningen University, Droevendaalsesteeg 1, 6708 PB Wageningen, the Netherlands

${ }^{2}$ Center for Plant Science Innovation, University of Nebraska, Lincoln, NE 68588, USA

${ }^{3}$ Department of Plant Pathology, University of Nebraska, Lincoln, NE 68588, USA

${ }^{4}$ Boyce Thompson Institute for Plant Research, Ithaca, NY 14853, USA

${ }^{5}$ Section of Plant Pathology and Plant-Microbe Biology, School of Integrative Plant Science, Cornell University, Ithaca, NY 14853, USA

'Present address: The State Key Laboratory of Plant Genomics, Institute of Genetics and Developmental Biology, Chinese Academy of Sciences, Beijing 100101, China

FPresent address: Center of Plant Molecular Biology (ZMBP), University of Tübingen, Auf der Morgenstelle 32, Tübingen D-72076, Germany 


\begin{abstract}
Receptor-like proteins (RLPs) and receptor-like kinases (RLKs) are cell surface receptors that are essential for detecting invading pathogens and subsequent activation of plant defence responses. RLPs lack a cytoplasmic kinase domain to trigger downstream signalling leading to host resistance. The RLK SOBIR1 constitutively interacts with the tomato RLP Cf-4, thereby providing $\mathrm{Cf}-4$ with a kinase domain. SOBIR1 is required for $\mathrm{Cf}-4$-mediated resistance to strains of the fungal tomato pathogen Cladosporium fulvum that secrete the effector Avr4. Upon perception of this effector by the Cf-4/SOBIR 1 complex, the central regulatory RLK Somatic Embryogenesis Receptor Kinase 3a (SERK3a) is recruited to the complex and defence signalling is triggered. SOBIR1 is also required for RLP-mediated resistance to bacterial, fungal and oomycete pathogens and we hypothesized that SOBIR1 is targeted by effectors of such pathogens to suppress host defence responses. In this study we show that Pseudomonas syringae pv. tomato DC3000 effector AvrPto interacts with Arabidopsis thaliana SOBIR1 and its orthologues of tomato and Nicotiana benthamiana, independent of SOBIR1 kinase activity. Interestingly, AvrPto suppresses Arabidopsis SOBIR1-induced cell death in N. benthamiana. Furthermore, AvrPto compromises Avr4-triggered cell death in Cf-4-transgenic N. benthamiana, without affecting $\mathrm{Cf}-4 / \mathrm{SOBIR} 1 / \mathrm{SERK} 3 a$ complex formation. Our study shows that the RLP co-receptor SOBIR1 is targeted by a bacterial effector, which results in compromised defence responses.
\end{abstract}




\section{Introduction}

The innate immune system of plants against invading pathogens consists of two layers, which are termed microbe-associated molecular pattern (MAMP)-triggered immunity (MTI) and effector-triggered immunity (ETI) (Janeway, 1989; Jones \& Dangl, 2006; Macho \& Zipfel, 2015; Couto \& Zipfel, 2016). MAMPs are conserved structural components of pathogens, whereas effectors are typically in plantainduced, secreted proteins of microbial pathogens. Effectors suppress plant defence responses, thereby causing effector-triggered susceptibility (ETS) (Jones \& Dangl, 2006; Dodds \& Rathjen, 2010; Feng \& Zhou, 2012). Essentially, there are two subcellular locations of pathogen perception: the apoplast and the cytoplasm (Cui et al., 2009; Dodds \& Rathjen, 2010; Zipfel, 2014). MAMPs and secreted effectors that end up in the apoplast are generally recognised by plasma membrane-associated pattern recognition receptors (PRRs) (Dodds \& Rathjen, 2010; Zipfel, 2014). Effectors that are translocated from fungal or oomycete haustoria, which are specialised feeding structures that are formed in the host cells, or injected into the cell by the type three secretion system (TTSS) of bacteria, are perceived by cytoplasmic immune receptors (Dodds \& Rathjen, 2010). These cytoplasmic receptors mostly carry a nucleotide binding site and leucine-rich repeats (NB-LRRs) (Dodds \& Rathjen, 2010).

PRRs are either receptor-like kinases (RLKs) or receptor-like proteins (RLPs) (Zipfel, 2014; Couto \& Zipfel, 2016). Compared to RLKs, RLPs lack a kinase domain to trigger downstream signalling. Recently it was observed that RLPs constitutively interact with the RLK Suppressor Of BAK1-Interacting RLK-1 (BIR1)-1 (SOBIR1) and require SOBIR1 for their function (Liebrand et al., 2013; Gust \& Felix, 2014; Liebrand et al., 2014). Arabidopsis thaliana (At, further referred to as Arabidopsis) SOBIR1 was originally identified as a suppressor of the bir 1-1 phenotype, partially rescuing bir 1 knockout plants that mount a constitutive defence response (Gao et al., 2009). AtSOBIR1 was found to function as a positive regulator of cell death, as overexpression of AtSOBIR 1 triggered enhanced basal defence and reduced colonisation by the bacterial pathogen Pseudomonas syringae pv. tomato (Pst) DC3000 (Gao et al., 2009). Recently, the RLK Somatic Embryogenesis Receptor Kinase 3 (SERK3)/Brassinosteroid-Insensitive 1 (BRI1)-Associated Receptor Kinase 1 (BAK1), further referred to as SERK3, has been reported as another suppressor of bir1-1 (Liu et al., 2016). Both SOBIR1 and SERK3 are required for bir1-1-triggered autoimmunity, and interaction was found between SOBIR1 and SERK3 in BIR1-silenced Arabidopsis (Liu et al., 2016).

The tomato (Solanum lycopersicum, Sl) RLP Cf-4 confers resistance to the pathogenic biotrophic fungus Cladosporium fulvum, by recognition of the secreted effector Avr4. It was recently shown that SERK3 is required for Cf-4 signalling and is recruited to the Cf-4/SOBIR1 complex upon recognition of Avr4 (Postma et al., 2016). Increasing evidence on signalling by RLP/SOBIR1/SERK3-containing complexes 
indicates that SOBIR1 constitutively forms a complex with RLPS, whereas SERK3 is specifically recruited to the RLP/SOBIR1 bipartite RLK upon ligand recognition by the RLP (Zhang et al., 2013a; Albert et al., 2015). SOBIR1 and SERK3 are also required for tomato Ve1-, I-, and Brassica napus LepR3-mediated resistance to Verticillium dahliae expressing Ave1 (Liebrand et al., 2013), Fusarium oxysporum f. sp. lycopersici expressing Avr1 (Catanzariti et al., 2017) and Leptosphaeria maculans expressing AvrLm1 (Ma \& Borhan, 2015), respectively, although for these particular RLPs the association of SERK3 with the RLP/SOBIR1 complex remains to be shown. SOBIR1 is also required for the functionality of various additional RLPs playing a role in immunity (Jehle et al., 2013; Zhang et al., 2014; Hegenauer et al., 2016), though the requirement of SERK3 for the functionality of these RLPs remains currently unknown. Furthermore, SOBIR1 is involved in resistance to the oomycete pathogen Phytophthora parasitica (Peng et al., 2015) and the fungus Magnaporthe oryzae (Takahashi et al., 2016).

The Arabidopsis RLK Flagellin-Sensing 2 (FLS2) confers resistance to Pst DC3000 (Zipfel et al., 2004). Flg22, a 22 amino acid peptide derived from a conserved domain of bacterial flagellin, is perceived by FLS2 (Gómez-Gómez \& Boller, 2000) together with SERK3 (Chinchilla et al., 2007; Heese et al., 2007; Sun et al., 2013). Recognition of flg22 triggers a rapid defence response, including calcium spiking (Boudsocq et al., 2010), reactive oxygen species (ROS) synthesis (Ma, 2014; Kadota et al., 2015), and mitogen-activated protein kinase (MAPK) activation (Asai et al., 2002). The receptorlike cytoplasmic kinase (RLCK) Botrytis-Induced Kinase 1 (BIK1) constitutively interacts with FLS2, and possibly with SERK3, and the association with FLS2 or SERK3 is not affected in single serk3- or fls2-knockout mutants, respectively (Lu et al., 2010; Zhang et al., 2010). However, flg22-induced BIK1 phosphorylation is both FLS2- and SERK3dependent, as BIK1 phosphorylation does not take place in fls2 or serk3 mutants (Lu et al., 2010). BIK1 is a substrate of SERK3, which in turn phosphorylates SERK3 and FLS2 (Lu et al., 2010). After flg22 perception, BIK1 is released from FLS2 and triggers early defence responses (Zhang et al., 2010; Ma, 2014).

Pathogenic bacteria inject their effectors into the host cells via the TTSS to suppress MTI by directly interfering with PRR function or with downstream signalling pathways (Lee et al., 2013; Couto \& Zipfel, 2016; Bi \& Zhou, 2017; Tang et al., 2017). For example, the effector AvrPto, also referred to as AvrPto1, is injected into host cells by Pst DC3000 and localizes to the plasma membrane (Shan et al., 2000). AvrPto targets FLS2 and suppresses the phosphorylation of its kinase domain (Xiang et al., 2008). AvrPto suppresses the flg22-activated MTI (He et al., 2006), although it is not clear if SERK3 is also targeted by AvrPto (Shan et al., 2008; Xiang et al., 2008; Xiang et al., 2011). Eventually, also BIK1 phosphorylation is suppressed, and the MAPK signalling pathway is inhibited (He et al., 2006; Lu et al., 2010; Xiang et al., 2011).

The resistance gene Pto (for resistance against Pst race 0 ) encodes an RLCK with serine-threonine specificity (Loh \& Martin, 1995), which competes with FLS2 
for AvrPto to trigger ETI (Xiang et al., 2008). In tomato, Pto confers resistance to Pst DC3000 carrying AvrPto (Martin et al., 1993) in a Pseudomonas resistance and fenthion (Prf)-dependent manner. Transient overexpression of AvrPto in Pto/Prftransgenic $N$. benthamiana causes a hypersensitive response (HR) (Salmeron et al., 1994; Scofield et al., 1996; Balmuth \& Rathjen, 2007). However, AvrPto does not affect association between Pto and Prf (Mucyn et al., 2006).

SOBIR1 is generally required for RLP-mediated resistance to bacterial, fungal, and oomycete pathogens (Jehle et al., 2013; Liebrand et al., 2013; Zhang et al., 2013a; Zhang et al., 2014; Albert et al., 2015; Ma \& Borhan, 2015; Peng et al., 2015; Hegenauer et al., 2016; Takahashi et al., 2016; Catanzariti et al., 2017). Therefore, we hypothesized that this regulatory co-receptor is targeted by cytoplasmic effectors of such pathogens in order to suppress host defence responses. Here we show that tomato SOBIR1, its homologue SISOBIR1-like, and its orthologues from Arabidopsis and $N$. benthamiana interact with AvrPto, independent of SOBIR1 kinase activity. In addition, AvrPto suppresses AtSOBIR1-induced constitutive immunity in $N$. benthamiana. Furthermore, AvrPto was found to suppress the Cf-4/Avr4-triggered $\mathrm{HR}$, without affecting Cf-4/SISOBIR1/SISERK3a complex formation.

\section{Results}

\section{AvrPto interacts with SOBIR1 in planta}

To investigate whether AvrPto and SOBIR1 interact, a binary construct containing C-terminally haemagglutinin ( $\mathrm{HA}$ ) epitope-tagged AvrPto was generated. AvrPto-HA was co-infiltrated with C-terminally tagged SISOBIR1-eGFP (Liebrand et al., 2013) in $N$. benthamiana by Agrobacterium-mediated transient expression (agro-infiltration). AvrPto-HA was also co-infiltrated with C-terminally tagged SISERK3a-eGFP as a positive control, because AtSERK3 was earlier reported to be targeted by AvrPto (Shan et al., 2008). Co-immunoprecipitation (co-IP) experiments reveal that AvrPtoHA co-purifies with SISOBIR1-eGFP upon pull-down of the latter, using GFP beads (Fig. 1A). Interaction between SISERK3a and AvrPto was also observed (Fig. 1A). In a reciprocal experiment in which SISOBIR1, SISERK3a and GUS were fused to HA and co-infiltrated with AvrPto-eGFP, a pull-down of SISOBIR1 and SISERK3a using HA beads also resulted in co-purification of AvrPto-eGFP (Fig. 1B).

In tomato, the SISOBIR1 homologue SISOBIR1-like shares a high similarity in amino acid sequence with SISOBIR1, and in N. benthamiana two clear SOBIR1 orthologues are present (Liebrand et al., 2013). In addition, the function of SOBIR1 appears to be highly conserved, as both AtSOBIR1 and SISOBIR1 interact with Cf-4 (Liebrand et al., 2013), and AtSOBIR1 is able to complement the loss of Avr4-triggered cell death in NbSOBIR1(-like)-silenced Cf-4 transgenic $N$. benthamiana plants (Liebrand 
et al., 2013; Bi et al., 2016). To study if the SOBIR1 homologue and its orthologues are also targeted by AvrPto, C-terminally eGFP-tagged SISOBIR1-like and NbSOBIR1 were generated and, together with SISOBIR1-eGFP and AtSOBIR1-eGFP (Liebrand et al., 2013; Bi et al., 2016), were co-infiltrated with AvrPto-HA in N. benthamiana. Co-IP experiments reveal that in all cases AvrPto co-purifies with the SOBIR1 variants (Fig. 2).
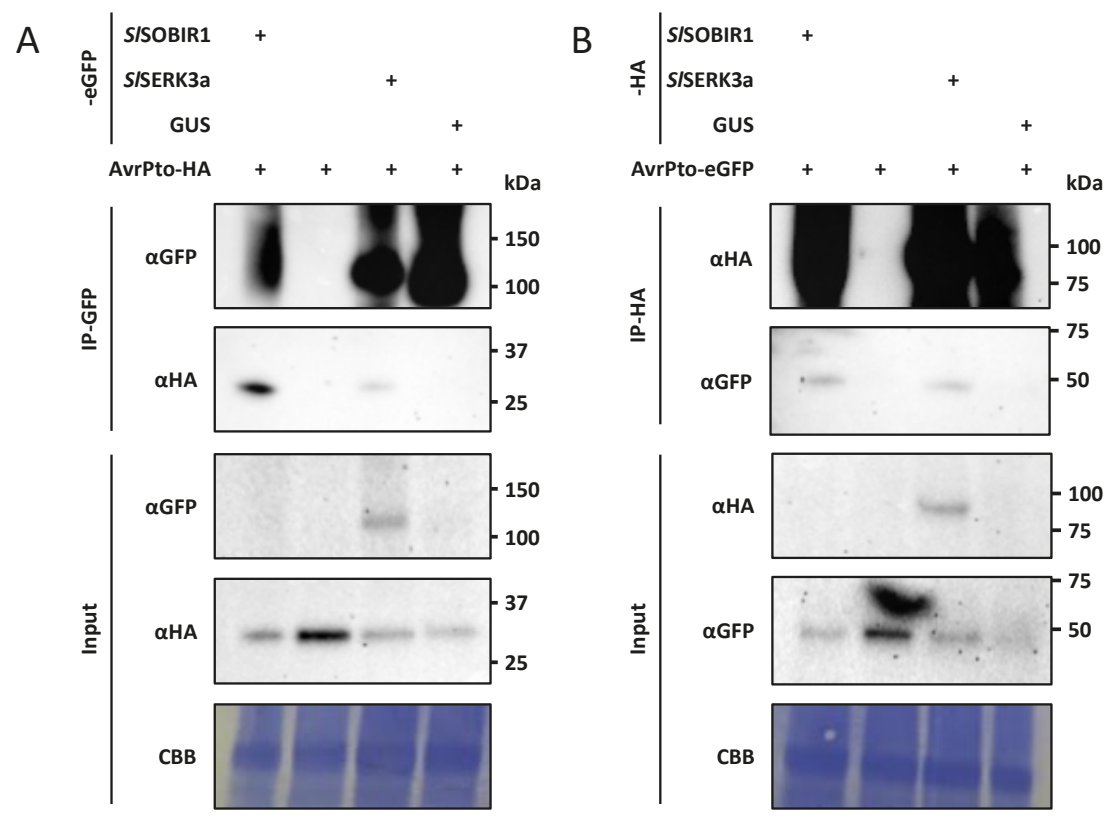

Figure 1. AvrPto interacts with SISOBIR1 in planta. (A) AvrPto-HA interacts with SISOBIR1eGFP. C-terminally eGFP-tagged SISOBIR1, SISERK3a and GUS were co-expressed with AvrPto-HA by agro-infiltration in $N$. benthamiana. Two days later, total proteins were extracted and subjected to immunoprecipitation (IP) using GFP_TrapA affinity beads. Total proteins (Input) and immunoprecipitated proteins (IP) were subjected to SDS-PAGE and blotted to PVDF membrane. Blots were probed with $\alpha$ GFP antibody to detect the immunoprecipitated eGFP-tagged proteins, and with $\alpha \mathrm{HA}$ antibody to detect co-precipitated AvrPto-HA. CBB, coomassie brilliant blue. Experiments were performed at least three times and representative results are shown. (B) AvrPto-eGFP interacts with SISOBIR1-HA and SISERK3aHA. C-terminally HA-tagged SISOBIR1, SISERK3a and GUS were co-expressed with AvrPto-eGFP in $N$. benthamiana. Two days later, total proteins were extracted and subjected to IP using HA magnetic beads. Total proteins (Input) and immunoprecipitated proteins (IP) were subjected to SDS-PAGE and blotted to PVDF membrane. Blots were probed with $\alpha \mathrm{HA}$ antibody to detect the immunoprecipitated HA-tagged proteins, and with aGFP antibody to detect co-precipitated AvrPto-eGFP. CBB, coomassie brilliant blue. Experiments were performed at least three times and representative results are shown. 


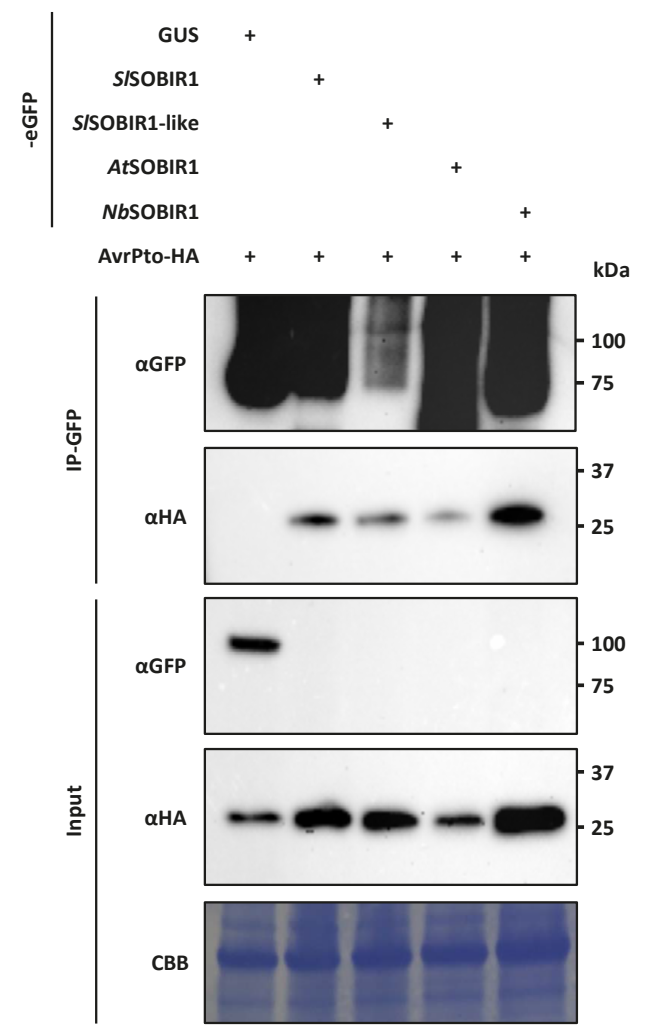

Figure 2. AvrPto interacts with SISOBIR1, SlSOBIR1-like, AtSOBIR1 and NbSOBIR1. C-terminally eGFP-tagged GUS, SISOBIR1, SISOBIR1-like, AtSOBIR1 and NbSOBIR1 were coexpressed with AvrPto-HA by agro-infiltration in $N$. benthamiana. Two days later, total proteins were extracted and subjected to IP using GFP TrapA affinity beads. Total proteins (Input) and immunoprecipitated proteins (IP) were subjected to SDS-PAGE and blotted to PVDF membrane. Blots were probed with $\alpha$ GFP antibody to detect the immunoprecipitated eGFP-tagged proteins, and with $\alpha \mathrm{HA}$ antibody to detect co-precipitated AvrPto-HA. Experiments were performed at least three times and representative results are shown.

\section{Kinase activity of SOBIR1 is not required for its interaction with AvrPto}

SOBIR1 is a so-called "RD" kinase and by its constitutive interaction with RLPs, bipartite RLKs are formed. In this bi-partite RLK, SOBIR1 is thought to provide the interacting RLP with a kinase domain to initiate defence signalling upon ligand recognition by the RLP (Gust \& Felix, 2014; Liebrand et al., 2014). It has been reported that AvrPto targets Pto and FLS2, while the interaction is dependent on kinase activity of these two targets (Xing et al., 2007; Xiang et al., 2008). To determine if kinase activity of SOBIR1 is required for its interaction with AvrPto, eGFP-tagged wild-type and kinase-dead SOBIR1 variants (Liebrand et al., 2013) (mutated in the catalytic aspartate (D) of the kinase domain) of Arabidopsis (AtSOBIR1 ${ }^{\text {D489N) }}$ and tomato SOBIR1 (SISOBIR1 ${ }^{\text {D473N)}}$ ) were co-infiltrated with AvrPto-HA. Co-IP experiments reveal that AvrPto co-purifies with all SOBIR1 variants upon their purification using GFP beads, indicating that kinase activity of SOBIR 1 is not required for its interaction with AvrPto (Fig. 3). 


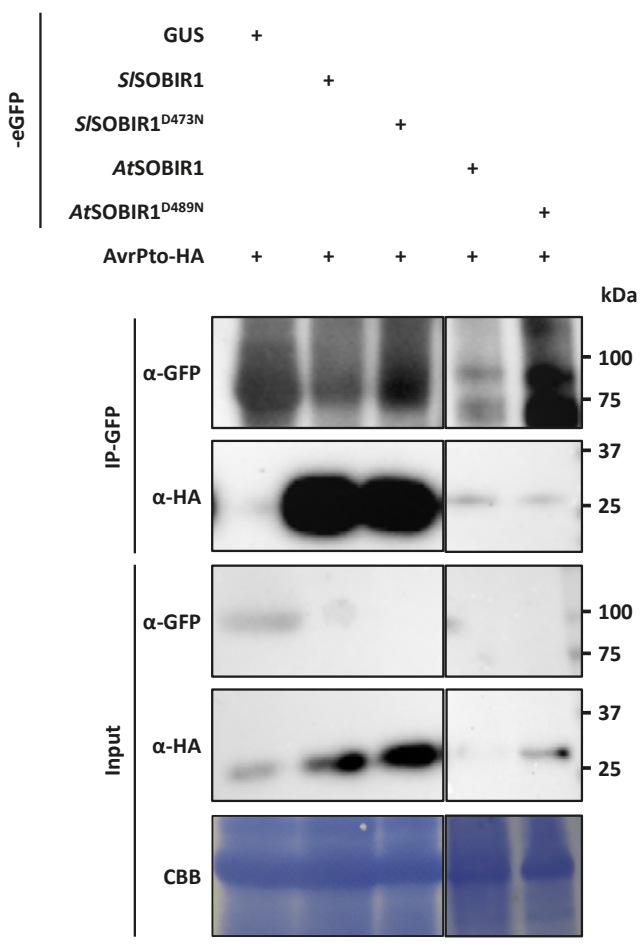

Figure 3. Kinase activity of SISOBIR1 and AtSOBIR1 is not required for their interaction with AvrPto. C-terminally eGFP-tagged GUS, SISOBIR1, kinase-dead SISOBIR1D473N, AtSOBIR1 and kinase-dead AtSOBIR1 ${ }^{\text {D489N }}$ were co-expressed with AvrPto-HA by agroinfiltration in $N$. benthamiana. Two days later, total proteins were extracted and subjected to immunoprecipitation using GFP_TrapA affinity beads. Total proteins (Input) and immunoprecipitated proteins (IP) were subjected to SDS-PAGE and blotted to PVDF membrane. Blots were probed with $\alpha$ GFP antibody to detect the immunoprecipitated eGFP-tagged proteins, and with $\alpha \mathrm{HA}$ antibody to detect co-precipitated AvrPto-HA. Experiments were performed at least three times and representative results are shown.

\section{Overexpression of AtSOBIR1 induces constitutive immunity, which requires a functional kinase domain}

SOBIR1 is a positive regulator of immunity (Gao et al., 2009), and we anticipated that the protein would constitutively induce an immune response, visualised as cell death, when it accumulates at relatively high levels. To investigate this, eGFP-tagged AtSOBIR1, SISOBIR1, SISOBIR1-like and NbSOBIR1 were transiently overexpressed in leaves of $N$. tabacum by agro-infiltration. Interestingly, only overexpression of AtSOBIR1 induced cell death, which was visible within two to three days after agro-infiltration (Fig. 4A). The other SOBIR1 variants that were tested did not exhibit this constitutive immunity symptom. Although protein accumulation was not tested in N. tabacum, the same constructs were expressed in $N$. benthamiana and resulted in clear protein accumulation for all constructs (discussed below) (Supplementary Fig. S1A). To determine if kinase activity of AtSOBIR1 is specifically required to induce constitutive immunity, eGFP-tagged AtSOBIR $1^{D 489 \mathrm{~N}}$ was transiently overexpressed in leaves of $N$. tabacum (Fig. 4B). This kinase-dead SOBIR1 mutant did not induce cell death upon its transient overexpression, indicating that a functional kinase domain is required for AtSOBIR1 to induce constitutive immunity in N. tabacum. This suggests that the constitutive immune response is triggered as a result of perturbation of the immune system of the plant, through constitutive activation of downstream immune signalling components. 
A

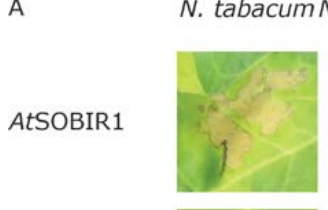

SISOBIR1
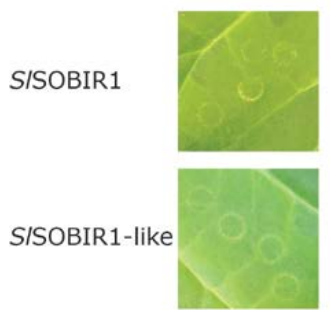

NbSOBIR1

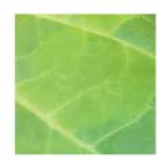

B
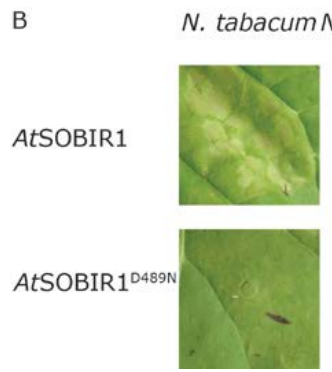
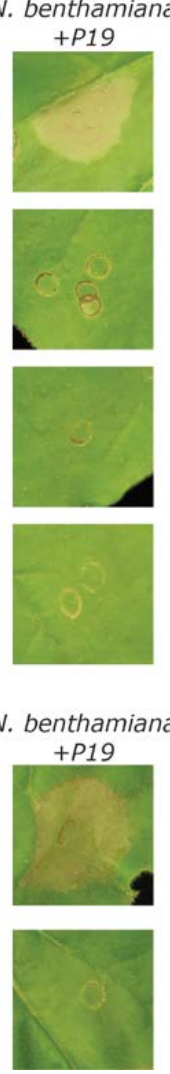

C
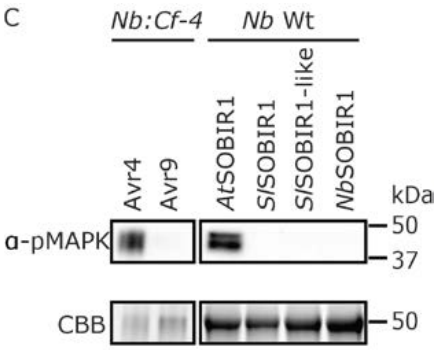

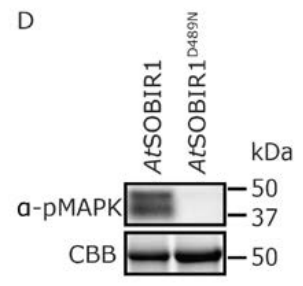

Figure 4. Constitutive immunity induced by overexpression of AtSOBIR1 is dependent on a functional kinase domain. (A) Transient expression of AtSOBIR1 induces cell death in N. tabacum and $N$. benthamiana. C-terminally eGFP-tagged AtSOBIR1, SISOBIR1, SISOBIR1-like and NbSOBIR1 were transiently expressed by agro-infiltration at an OD of 1 ; in $N$. benthamiana all constructs were co-expressed with the silencing suppressor P19 at an OD of 1. Pictures were taken at 2 days post-infiltration (dpi) for $N$. tabacum and at 3 dpi for N. benthamiana. See also Fig. S1A. (B) Transient expression of C-terminally eGFP-tagged kinase-dead AtSOBIR1 ${ }^{D 489 N}$ does not induce cell death in N. tabacum or N. benthamiana. The experiment was performed as described for panel A. See also Fig. S1A. (C) MAPKs are activated upon Avr4 recognition by $\mathrm{Cf}-4$, and upon overexpression of AtSOBIR1. Left, $2 \mu \mathrm{M}$ of Avr4 or Avr9 protein was infiltrated in $N$. benthamiana:Cf-4 plants and after 15 minutes total protein was extracted and analysed for MAPK activation using anti-p42/p44-erk antibody. Right, C-terminally eGFP-tagged AtSOBIR1, SISOBIR1, SISOBIR1-like and NbSOBIR1 were transiently co-expressed with P19 in N. benthamiana. At 2 dpi total protein was extracted and analysed for MAPK activation using anti-p42/p44-ERK antibody. (D) MAPKs

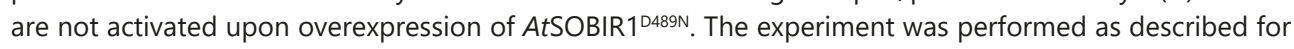
panel D. Experiments were performed at least three times and representative results are shown.

The same constructs were tested for constitutive immune activation in $N$. benthamiana. No cell death was observed for any of the SOBIR1 variants when expressed alone (data not shown), although all proteins do accumulate (Supplementary Fig. S1A and B). However, when co-expressed with the silencing suppressor P19 
(Voinnet et al., 2015), cell death could be observed for AtSOBIR1 at two to three days after infiltration, and again not for any of the other tested SOBIR1 variants (Fig. 4A, Supplementary Fig. S1). N. tabacum is generally more sensitive to overexpression of immunity-related proteins, and probably therefore does not require co-expression of a silencing suppressor to provoke AtSOBIR1 constitutive immunity (van der Hoorn et al., 2000; Zhang et al., 2013b).

To obtain additional support that this cell death phenotype indeed reflects an immune response, we analysed MAPK activation, which is a key downstream step in defence activation (Stulemeijer et al., 2007; Xu et al., 2014). Upon Avr4 recognition by $\mathrm{Cf}-4$ in stable transgenic $N$. benthamiana expressing tomato $C f-4$, immune blots showed a typical MAPK activation pattern (Fig. 4C). Notably, induction of cell death upon AtSOBIR1 overexpression in $N$. benthamiana also coincided with MAPK activation (Fig. 4C and 4D). In conclusion, these data show that the cell death caused by AtSOBIR1 is dependent on its kinase activity, and represents a constitutive immune response.

\section{AvrPto suppresses AtSOBIR1-induced constitutive immunity}

To determine whether AvrPto is able to suppress the cell death response induced by overexpression of AtSOBIR1, AvrPto-eGFP and GUS-eGFP were co-infiltrated with AtSOBIR1-eGFP and P19 in N. benthamiana. Co-infiltration of GUS-eGFP does not affect AtSOBIR1-induced cell death, whereas the cell death response is strongly suppressed by AvrPto-eGFP, indicating that AtSOBIR1-induced constitutive immunity is suppressed by AvrPto (Fig. 5).

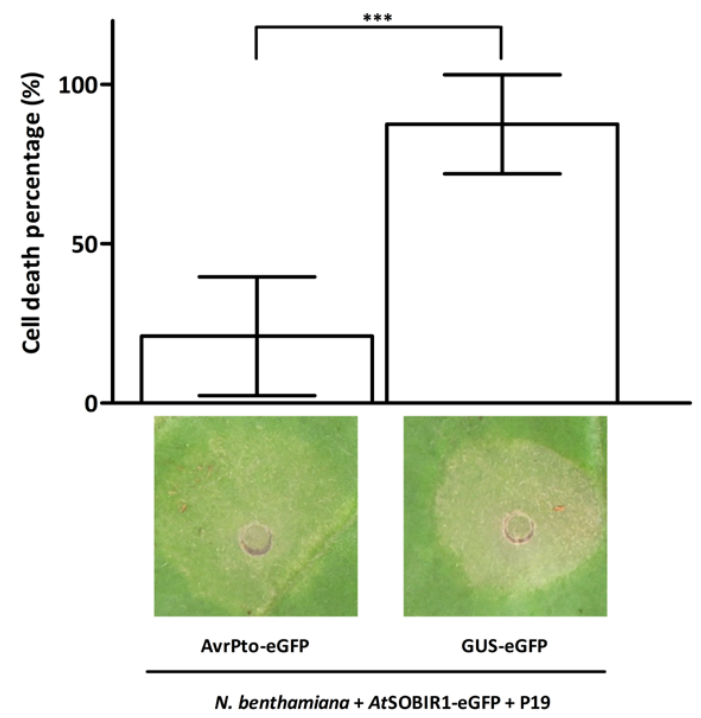

Figure 5. Constitutive immunity induced by overexpression of AtSOBIR1 is suppressed by AvrPto. AtSOBIR1-eGFP and P19 were co-expressed with GUS-eGFP or AvrPtoeGFP by agro-infiltration in $N$. benthamiana at an $O D$ of 1 . Pictures were taken at $4 \mathrm{dpi}$. Cell death at $4 \mathrm{dpi}$ was scored by its intensity. The means of the cell death percentages are shown. Asterisks indicate a statistically significant difference according to a Student's $t$-test (*** $P<0.0001$ ), with $\mathrm{n}=20$. Experiments were performed at least three times and representative results are shown. 


\section{AvrPto suppresses the Avr4-triggered cell death in $\mathbf{N}$. benthamiana:Cf-4 plants}

SOBIR1 is required for the $\mathrm{Cf}-4$-mediated cell death upon recognition of Avr4, and for resistance to C. fulvum (Liebrand et al., 2013). Furthermore, kinase activity of SOBIR1 is necessary for $\mathrm{Cf}-4$ function as a kinase-inactive variant of AtSOBIR1 fails to complement the loss of Avr4-triggered cell death in NbSOBIR1(-like)-silenced $N$. benthamiana:Cf-4 plants (Liebrand et al., 2013; Bi et al., 2016).

To study if AvrPto is able to suppress the Avr4-triggered cell death through suppression of SOBIR1-mediated defence signalling, AvrPto-eGFP and GUS-eGFP were transiently overexpressed in $N$. benthamiana:Cf-4 plants, two days before agro-infiltration of Avr4. Figure 6 shows that cell death triggered by Avr4 is strongly suppressed upon infiltration of AvrPto-eGFP, when compared to infiltration of GUSeGFP. Together with the observation that AvrPto suppresses AtSOBIR1-induced constitutive immunity (Fig. 5), the suppression of Avr4-triggered cell death by AvrPto indicates that AvrPto compromises Avr4-triggered cell death through suppression of SOBIR1-mediated defence signalling.

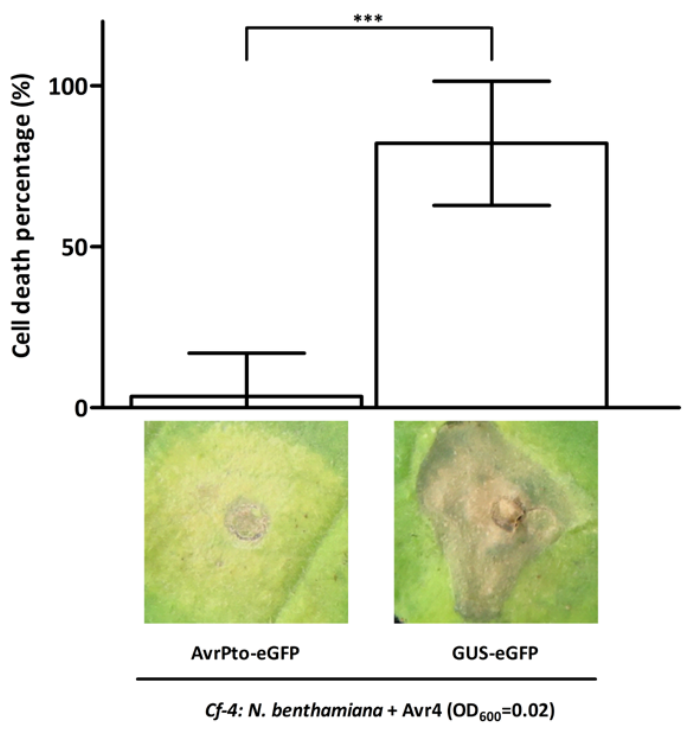

Figure 6. The Avr4-triggered cell death in $N$. benthamiana:Cf-4 plants is suppressed by AvrPto. AvrPto-eGFP and GUS-eGFP were expressed at an OD of 1 in N. benthamiana:Cf-4 plants at two days before agro-infiltration of Avr4 at an OD of 0.02. Pictures were taken at 5 dpi of Avr4 infiltration. Cell death at 5 dpi was scored by the intensity. The means of the cell death percentages are shown. Asterisks indicate a statistically significant difference according to a Student's $t$-test (*** $P<0.0001$ ), with $n=15$. Experiments were performed at least three times and representative results are shown.

\section{AvrPto does not affect Cf-4/SISOBIR1/SISERK3a complex formation}

Recently it was shown that SISERK3a is involved in the Cf-4-triggered signalling pathway (Postma et al., 2016). SOBIR1 constitutively interacts with Cf-4 (Liebrand et al., 2013), whereas SISERK3a interacts with Cf-4 in an Avr4-dependent manner, as SISERK3a is specifically recruited to the Cf-4/SISOBIR1 complex in the presence of Avr4 (Postma et al., 2016). Silencing of either NbSOBIR1(-like) or NbSERK3a/b 
compromises the Avr4-triggered cell death (Liebrand et al., 2013; Postma et al., 2016).

To study the effect of AvrPto on Cf-4/SISOBIR1/SISERK3a complex formation, AvrPto-HA was co-infiltrated with Cf-4-eGFP, SISOBIR1-HA and SISERK3a-Myc, followed by infiltration of Avr4 protein. Figure 7 shows that Cf-4-eGFP and SISOBIR1$\mathrm{HA}$ are invisible in the input, but are readily detectable in the immunoprecipitate, which is consistent with our earlier published studies (Liebrand et al., 2013; Postma et al., 2016). Co-IP experiments reveal that AvrPto does not affect the interaction between Cf-4 and SISOBIR1. Moreover, the recruitment of SISERK3a to the Cf-4/ SISOBIR1 complex upon infiltration of Avr4 protein is not affected (Fig. 7). These results indicate that AvrPto does not suppress the Avr4-triggered cell death by hampering Cf-4/SISOBIR1/SISERK3a complex formation upon Avr4 recognition by Cf-4.

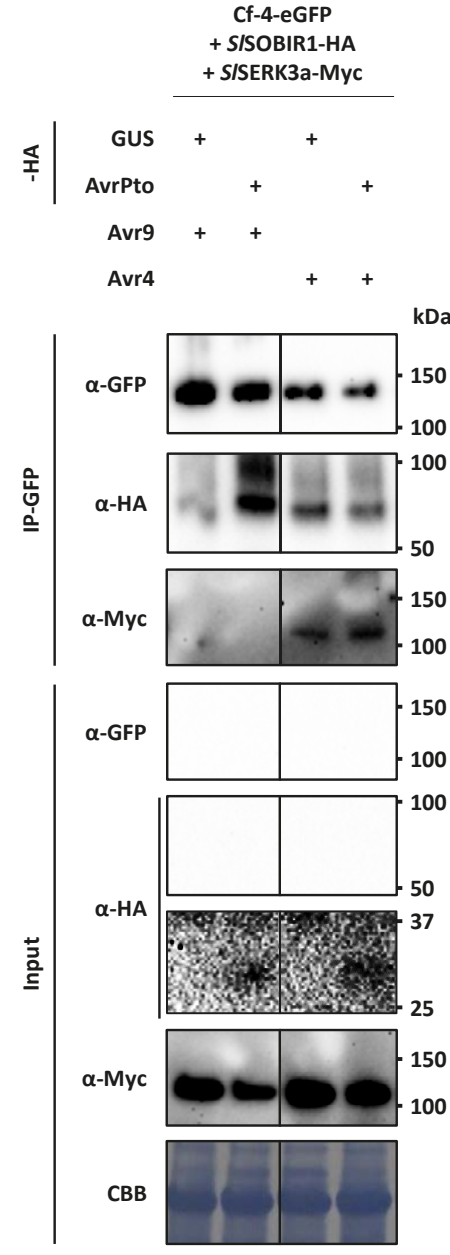

Figure 7. Avr4-mediated SISERK3a recruitment to the Cf-4/SISOBIR1 complex is not affected by AvrPto. Cf-4eGFP, SISOBIR1-HA and SISERK3a-Myc were co-expressed with either GUS-HA or AvrPto-HA by agro-infiltration in $N$. benthamiana. Two days later, Avr4 or Avr9 protein $(2 \mu \mathrm{M})$ was infiltrated in the same area and leaves were harvested 30 min later. Total proteins were extracted and subjected to IP using GFP_TrapA affinity beads. Total proteins (Input) and immunoprecipitated proteins (IP) were subjected to SDSPAGE and blotted to PVDF membrane. Blots were probed with $\alpha$ GFP antibody to detect immunoprecipitated Cf-4eGFP, with $\alpha H A$ antibody to detect co-precipitated SISOBIR1$\mathrm{HA}$, and with $\alpha \mathrm{Myc}$ antibody to detect co-precipitated SISERK3a-Myc. The lower $\alpha H A$ panel from the input shows an overexposed blot to reveal accumulation of AvrPto-HA. CBB, coomassie brilliant blue. Experiments were performed at least three times and representative results are shown. 


\section{Discussion}

In this study, we provide evidence that AvrPto interacts with AtSOBIR1 and its orthologues of tomato and $N$. benthamiana, independent of the kinase activity of SOBIR1. Overexpression of AtSOBIR1 induces constitutive immunity, which is suppressed by AvrPto. In addition, AvrPto also suppresses Cf-4/Avr4-triggered cell death without affecting Cf-4/SISOBIR1/SISERK3a complex formation.

\section{AtSOBIR1 induces a constitutive immune response}

AtSOBIR1 is a functional orthologue of NbSOBIR1 (Liebrand et al., 2013) and, in contrast to NbSOBIR1 or SISOBIR1, overexpression of AtSOBIR1 in N. tabacum or N. benthamiana induces constitutive immunity, which is observed as cell death (Fig. 4A). This observation is in agreement with the cell death observed upon overexpression of AtSOBIR 1 in Arabidopsis (Gao et al., 2009). The lack of constitutive immune activity of $N b S O B I R 1$ and SISOBIR1 could be an effect of SOBIR1 signalling regulation in $N$. tabacum and $N$. benthamiana. The signalling capacity of immune receptors is tightly regulated, as immune homeostasis has to be maintained and its de-regulation may be lethal (Couto \& Zipfel, 2016).

A way of regulating immune signalling activity is at the level of phosphorylation of the kinase domain of the signalling components. For instance, the Arabidopsis phosphatases PP2A and PP2C38, associating with SERK3 and BIK1 respectively, were found to negatively regulate the activity of their target kinases (Segonzac et al., 2014; Couto et al., 2016). Potential intrinsic differences between phosphatases from Arabidopsis and Solanaceous plants, involved in negatively regulating SOBIR1 signalling, could explain the lack of constitutive immune activity of SISOBIR1 or NbSOBIR1, in contrast to AtSOBIR1, when expressed in Solanaceous plants. It is likely that Solanaceous plants can fine-tune the activity of endogenous versions of SOBIR1 in a more controlled manner than the activity of heterologously expressed AtSOBIR1. Thus, it could be that endogenous phosphatases of N. tabacum and N. benthamiana can properly negatively regulate Solanaceous SOBIR1 immune signalling, whereas these phosphatases might for example have lower affinity for the more distantly related orthologue AtSOBIR1. This could result in a higher basal level of phosphorylation of the kinase domain of AtSOBIR1. This higher level of phosphorylation might perturb the immune system of the plant and trigger a constitutive immune response in $N$. tabacum and $N$. benthamiana, which is also reflected by constitutive activation of MAPK signalling (Fig. 4C). 


\section{AvrPto suppresses $\mathrm{Cf}-4 /$ SISOBIR1/SISERK3a-triggered cell death, without affecting complex formation}

AvrPto has been reported to suppress cell death triggered by Pst strain T1 in N. benthamiana (Kang et al., 2004). In addition, AvrPto has been shown to suppress the flg22/elf18/elf26-triggered immune response. Elf18 and elf26 are fragments of the bacterial MAMP Elongation Factor-Temperature Unstable (EF-Tu) that are perceived by the RLK EF-Tu Receptor (EFR) (He et al., 2006; Zipfel et al., 2006; Xiang et al., 2008). Here we show that AvrPto is able to suppress AtSOBIR1-induced constitutive immunity (Fig. 5), as well as the Cf-4-mediated cell death response triggered by Avr4 (Fig. 6). Previously, it was reported that AvrPto fails to suppress the cell death triggered by Cf-9/Avr9 (Kang et al., 2004). Cf-9 also interacts with SOBIR1 (Liebrand et al., 2013) and we anticipate that Cf-9 requires SOBIR1 for its function. The absence of AvrPto-mediated suppression of Cf-9/Avr9-induced cell death might be caused by the use of a lower amount of Agrobacterium expressing AvrPto $\left(\mathrm{OD}_{600}=0.4\right.$, as compared to $O D=1.0$ in our studies). Furthermore, Kang et al. (2004) co-infiltrated AvrPto with Cf-9/Avr9, whereas in our study AvrPto was infiltrated two days before infiltration of Avr4 in Cf-4 transgenic N. benthamiana.

It has been shown that AvrPtoB, which is another TTSS effector of Pst DC3000 (Pedley \& Martin, 2003), and AvrPto both interact with FLS2 and SERK3, whereas only the association of AvrPtoB and FLS2 gets stronger upon flg22 treatment (Gohre et al., 2008; Shan et al., 2008; Xiang et al., 2008). In addition, the flg22-induced FLS2SERK3 interaction is suppressed by both AvrPtoB and AvrPto (Shan et al., 2008). Furthermore, the dissociation of BIK1 from FLS2 and SERK3 in the presence of flg22 is also suppressed by AvrPto (Lu et al., 2010; Zhang et al., 2010). In view of developmental regulation, SERK3 is involved in the Brassinosteroid (BR)-triggered signalling pathway, since serk3 mutants show reduced sensitivity to BR (Li et al., 2002). SERK3 constitutively interacts with the BR receptor BRI1 (Li et al., 2002), and this interaction is also suppressed by AvrPto (Shan et al., 2008).

Altogether, these observations indicate that AvrPto suppresses flg22-triggered plant immunity by interrupting PRR complex formation with the essential co-receptor SERK3, and suppresses BRI1 and SERK3 complex formation involved in BR-mediated developmental regulation (Shan et al., 2008). In this study, we show that AvrPto is able to suppress $\mathrm{Cf}-4$ /Avr4-triggered cell death, without affecting the constitutive interaction between $\mathrm{Cf}-4 / S$ SSOBIR1 and the Avr4-dependent recruitment of SISERK3a to the Cf-4/SISOBIR1 complex (Fig. 6 and 7). As we found that AvrPto interacts with both SISOBIR1 and SISERK3 (Fig. 1), in this case AvrPto might interfere in the phosphorylation status of SOBIR1 and/or SERK3 to suppress Cf-4/Avr4-triggered cell death (Fig. 6). 


\section{TTSS effectors interfere in host protein phosphorylation}

Effector-mediated modifications of defence-related proteins have been shown to be an effective way to suppress the immune response (Lee et al., 2013). Interestingly, it has been shown that effectors interfere with the phosphorylation status of important host kinase proteins, including PRRs, RLCKs and MPKs (Lee et al., 2013; Macho \& Zipfel, 2015; Couto \& Zipfel, 2016; Bi \& Zhou, 2017; Tang et al., 2017). For example, RPM1-Interacting Protein 4 (RIN4) associates with, and is required for, Resistance to Pseudomonas Syringae pv Maculicola 1 (RPM1)-mediated resistance (Mackey et al., 2002). The P. syringae effector AvrB interacts with RIN4 and induces its phosphorylation, thereby activating the RPM1 signalling pathway leading to resistance to the bacterium (Mackey et al., 2002; Lee et al., 2015). Similar to AvrB, AvrRpm1 also induces RIN4 phosphorylation (Mackey et al., 2002).

Pseudomonas HopAO family members all have tyrosine phosphatase activity, which is required for pathogen virulence (Bretz et al., 2003; Castaneda-Ojeda et al., 2017). For instance, the phosphatase catalytic activity of HopAO1 is required for suppressing callose deposition and MAPK activation upon bacterial colonisation of Arabidopsis (Underwood et al., 2007; Macho et al., 2014; Castaneda-Ojeda et al., 2017). Recently, HopAO1 was reported to dephosphorylate EFR on an essential tyrosine residue, a modification occurring upon elf18 treatment, without interfering in the EFR-SERK3 interaction (Macho et al., 2014). De-phosphorylation of this particular tyrosine residue was found to result in suppression of MTI (Macho et al., 2014).

In another study it was found that SERK3, which interacts with FLS2 and EFR upon defence activation, is targeted by HopF2, AvrPtoB and AvrPto (Yasuda et al., 2017). HopF2 targets SERK3 to suppress BIK1 phosphorylation, thereby compromising MAPK activation (Wu et al., 2011; Zhou et al., 2014).

AvrPtoB targets Arabidopsis LysM-RLK Chitin Receptor Kinase 1 (CERK1) in order to degrade the protein, and this effector also seems to suppress CERK1 kinase activity (Gimenez-lbanez et al., 2009). In addition, kinase activity of the AvrPtoB tomato-interacting 9 (Bti9) protein, the closest tomato homologue of CERK1, is also suppressed by AvrPtoB (Zeng et al., 2012). AvrPtoB triggers degradation of FLS2/ EFR, but does not directly affect their kinase activity (Gohre et al., 2008), which is in contrast to SERK3 which is not degraded but inhibited in its kinase activity (Cheng et al., 2011).

Cytoplasmic RLCKs, which play a role downstream of PRRs, are phosphorylated by ligand-activated PRRs and in their turn trans-phosphorylate the kinase domain of these PRRs (Couto \& Zipfel, 2016). Phosphorylation of the RLCK AvrPphB-Susceptible 1 (PBS1) (Swiderski \& Innes, 2001) is required for its interaction with Resistance to Pseudomonas Syringae 5 (RPS5) (Warren et al., 1998; Ade et al., 2007). PBS1 is cleaved by HopAR1 (AvrPphB) from Pst DC3000 to activate RPS5-mediated auto immunity 
(Shao et al., 2003; Ade et al., 2007). HopAR1 also dephosphorylates BIK1 to suppress the flg22-induced signalling pathway (Zhang et al., 2010). In addition, BIK1 is also dephosphorylated by the Xanthomonas TTSS effector AvrAC (Feng et al., 2012).

Activation of the MAPK cascade is a common downstream event in plant resistance to pathogens, and the kinase activity of these MAPKs is modified by several bacterial effectors (Lee et al., 2013). HopF2 interacts with MKK5 to prevent its autophosphorylation (Wang et al., 2010), and HopAl1 targets MPK3, MPK6 and MPK4 to dephosphorylate them (Zhang et al., 2007; Zhang et al., 2012).

AvrPto has many targets, including SERK3, FLS2, EFR, CERK1, and Pto. Recently, it was reported that both AvrPto overexpression and a double mutation in both SERK3 and SERK4 (also referred to as BAK1-Like1 (BKK1)), similarly suppress oligogalacturonide (OG)-induced defence signalling (Gravino et al., 2017). However, it is not clear if AvrPto is able to directly suppress SERK3 kinase activity. AvrPto binds to the kinase domain of FLS2 and EFR, thereby acting as a kinase inhibitor and blocking FLS2 and EFR autophosphorylation to compromise flg22/elf26-triggered immunity (Xiang et al., 2008). Here we show that AvrPto interacts with SOBIR1, however it is not known whether AvrPto directly binds to the kinase domain of SOBIR1 or whether this interaction occurs indirectly, through another protein that interacts with the SOBIR1 kinase domain. Furthermore, AvrPto targets Pto, thereby suppressing Pto kinase activity (Xing et al., 2007). Surprisingly, the association between BRI1 and SERK3 is interrupted by AvrPto, but the phosphorylation of BRI1 and SERK3 upon BR treatment remains unaffected (Shan et al., 2008).

The overview provided above shows that, except for the BR-triggered phosphorylation of BRI1 and SERK3 that is not affected by AvrPto, the level of phosphorylation of the AvrPto-targeted kinases is lowered by AvrPto. Although there is a debate on the interaction between AvrPto and SERK3 (Xiang et al., 2011), we found that AvrPto targets both SOBIR1 and SERK3 (Fig. 1). Therefore, we anticipate that AvrPto might play a role in altering the phosphorylation status of SOBIR1 and/ or SERK3 to suppress Cf-4/Avr4-triggered cell death (Fig. 6), without affecting Cf-4/ SISOBIR1/SISERK3a complex formation (Fig. 7). Changing the phosphorylation status of the kinase domain of SOBIR1 and/or SERK3 could be the result of inhibition of the kinase activity of these RLKs through their targeting by AvrPto.

\section{The requirement of kinase activity of targeted proteins for their interaction with effectors}

Targeting of host kinases by pathogen effectors is a common strategy to suppress MTI. In most cases, the kinase domain itself is targeted and kinase activity of the targeted proteins plays a role in the interaction. For example, AvrAC interacts with BIK1 to uridylylate the BIK1 phosphorylation site (Feng et al., 2012). Kinase activity 
of BIK1 is not required for the interaction, but it affects the uridylylation by AvrAC (Feng et al., 2012).

As mentioned above, AvrPtoB targets the kinase domain of SERK3 (Shan et al., 2008), Pto (Kim et al., 2002), FLS2 (Gohre et al., 2008), CERK1 (Gimenez-lbanez et al., 2009) and Bti9 (Zeng et al., 2012). Kinase activity of SERK3 (Cheng et al., 2011) and Pto (Dong et al., 2009) is required for the interaction with AvrPtoB. However, whether kinase activity is required for interaction with FLS2, CERK1 and Bti9 is unknown.

Kinase activity of Pto is required for its interaction with AvrPto, as it has been shown that the mutant Pto ${ }^{T 204 N}$, which has low kinase activity, only weakly interacts with AvrPto, whereas the kinase-inactive variant Pto ${ }^{\mathrm{S226D}}$ completely loses its interaction with the effector (Xing et al., 2007). Threonine 204 of Pto is highly conserved among the kinase domains of SERK3, SERK4, FLS2 and EFR (Shan et al., 2008). In SERK3, threonine 455 is equivalent to threonine 204 of Pto and a SERK3 ${ }^{\text {T455N }}$ mutant has reduced kinase activity (Lin et al., 2014). However this substitution in SERK3 does not affect its interaction with AvrPto (Shan et al., 2008). It has been shown that Pto has evolved to compete for AvrPto interaction with FLS2 through its ATP-binding site (Xing et al., 2007), and has higher affinity for AvrPto than FLS2 (Xiang et al., 2008). AvrPto targets the ATP-binding site in the kinase domain of FLS2, and a mutation $(\mathrm{K} 898 \mathrm{H})$ in the ATP-binding site of FLS2, which is anticipated to have a lower kinase activity, abolishes its interaction with AvrPto (Xing et al., 2007; Xiang et al., 2008). Although it is unknown whether the kinase domain of SOBIR1 by itself is sufficient for interaction with AvrPto, kinase activity of SOBIR1 appears not to be required (Fig. 3). This observation reveals different requirements for the interaction of AvrPto with its targets, indicating that AvrPto utilizes different mechanisms to target and affect plant kinases involved in defence signalling.

\section{Materials \& Methods}

\section{Plant materials and growth conditions}

Nicotiana benthamiana, Cf-4-transgenic N. benthamiana:Cf-4 (Gabriëls et al., 2007) and $N$. tabacum were grown under $16 \mathrm{~h}$ of light at $25^{\circ} \mathrm{C}$ and $8 \mathrm{~h}$ of darkness at $21^{\circ} \mathrm{C}$ in climate chambers with a relative humidity of $75 \%$.

\section{Binary vectors for Agrobacterium infiltrations (agro-infiltrations)}

A fragment consisting of the AvrPto coding sequence in an entry vector was transferred to the destination vector pBIN-KS-35S::GWY-eGFP (SOL 2095; for C-terminally tagging with eGFP), to generate AvrPto-eGFP, and pGWB14-35S::GWYHA (SOL 2749; for C-terminally tagging with the HA epitope), to generate AvrPto-HA. 
SISERK3a-HA，SISOBIR1-HA，SISOBIR1-like-eGFP，SISOBIR1 1473N_eGFP，NbSOBIR1eGFP, GUS-eGFP and GUS-HA were generated from entry vectors which have been described previously (Liebrand et al., 2013; Bi et al., 2016). SISERK3a-eGFP, SISOBIR1-

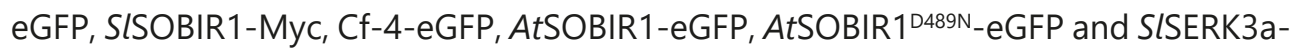
Myc have been described previously (Liebrand et al., 2013; Bi et al., 2016; Postma et al., 2016). Silencing suppressor P19, which was included in all co-IP experiments, has been described previously (Voinnet et al., 2015).

Binary vectors were transformed to Agrobacterium tumefaciens strain C58C1, carrying helper plasmid pCH32. Infiltration of Agrobacterium into plant leaves was performed as described at an $\mathrm{OD}_{600}$ of 1 , unless indicated otherwise (van der Hoorn et al., 2000).

\section{Co-immunoprecipitations (co-IPs) and immunoblotting}

Co-IPs were performed as described previously (Liebrand et al., 2012). The following antibodies were used: anti-p42/p44-ERK (New England Biolabs), in combination with goat anti-rabbit (Sigma) as a secondary antibody, anti-GFP (Miltenyi Biotec $\mathrm{GmbH}$ ), anti-HA (clone 3F10; Roche Applied Science), anti-cMyc (9E10; Santa Cruz Biotechnology), with sheep anti-mouse (Amersham) as a secondary antibody.

\section{Acknowledgements}

T.W.H. Liebrand and R.R. Bye are acknowledged for initiating experiments on AtSOBIR1-induced constitutive cell death in N. tabacum. L. Deurhof is acknowledged for technical support. J. Wu and G. Bi are supported by the China Scholarship Council (CSC). A.M. van der Burgh is supported by the Netherlands Organization for Scientific Research (NWO), division Earth and Life Sciences (ALW). 


\section{References}

Ade, J., DeYoung, B.J., Golstein, C., and Innes, R.W. 2007. Indirect activation of a plant nucleotide binding site-leucine-rich repeat protein by a bacterial protease. Proc. Natl. Acad. Sci. U.S.A. 104:2531-25366.

Albert, I., Bohm, H., Albert, M., Feiler, C.E., Imkampe, J., Wallmeroth, N., Brancato, C., Raaymakers, T.M., Oome, S., Zhang, H., Krol, E., Grefen, C., Gust, A.A., Chai, J., Hedrich, R., Van den Ackerveken, G., and Nurnberger, T. 2015. An RLP23-SOBIR1-BAK1 complex mediates NLP-triggered immunity. Nat. Plants 1:15140-15148.

Asai, T., Tena, G., Plotnikova, J., Willmann, M.R., Chiu, W.L., Gomez-Gomez, L., Boller, T., Ausubel, F.M., and Sheen, J. 2002. MAP kinase signalling cascade in Arabidopsis innate immunity. Nature 415:977-983.

Balmuth, A., and Rathjen, J.P. 2007. Genetic and molecular requirements for function of the Pto/Prf effector recognition complex in tomato and Nicotiana benthamiana. Plant J. 51:978-990.

Bi, G., and Zhou, J.M. 2017. MAP kinase signaling pathways: a hub of plant-microbe interactions. Cell Host Microbe 21:270-273.

Bi, G., Liebrand, T.W.H., Bye, R.R., Postma, J., van der Burgh, A.M., Robatzek, S., Xu, X., and Joosten, M.H.A.J. 2016. SOBIR1 requires the GxxxG dimerization motif in its transmembrane domain to form constitutive complexes with receptor-like proteins. Mol. Plant Pathol. 17:96-107.

Boudsocq, M., Willmann, M.R., McCormack, M., Lee, H., Shan, L., He, P., Bush, J., Cheng, S.H., and Sheen, J. 2010. Differential innate immune signalling via $\mathrm{Ca}^{2+}$ sensor protein kinases. Nature 464:418-422.

Bretz, J.R., Mock, N.M., Charity, J.C., Zeyad, S., Baker, C.J., and Hutcheson, S.W. 2003. A translocated protein tyrosine phosphatase of Pseudomonas syringae pv. tomato DC3000 modulates plant defence response to infection. Mol. Microbiol. 49:389-400.

Castaneda-Ojeda, M.P., Moreno-Perez, A., Ramos, C., and Lopez-Solanilla, E. 2017. Suppression of plant immune responses by the Pseudomonas savastanoi pv. savastanoi NCPPB 3335 type III effector tyrosine phosphatases HopAO1 and HopAO2. Front. Plant Sci. 8:680.

Catanzariti, A.M., Do, H.T., Bru, P., de Sain, M., Thatcher, L.F., Rep, M., and Jones, D.A. 2017. The tomato I gene for Fusarium wilt resistance encodes an atypical leucine-rich repeat receptor-like protein whose function is nevertheless dependent on SOBIR1 and SERK3/BAK1. Plant J. 89:1195-1209.

Cheng, W., Munkvold, K.R., Gao, H., Mathieu, J., Schwizer, S., Wang, S., Yan, Y.B., Wang, J., Martin, G.B., and Chai, J. 2011. Structural analysis of Pseudomonas syringae AvrPtoB bound to host BAK1 reveals two similar kinase-interacting domains in a type III Effector. Cell Host Microbe 10:616-626.

Chinchilla, D., Zipfel, C., Robatzek, S., Kemmerling, B., Nurnberger, T., Jones, J.D., Felix, G., and Boller, T. 2007. A flagellin-induced complex of the receptor FLS2 and BAK1 initiates plant defence. Nature 448:497-500.

Couto, D., and Zipfel, C. 2016. Regulation of pattern recognition receptor signalling in plants. Nat. Rev. Immunol. 16:537-552.

Couto, D., Niebergall, R., Liang, X., Bucherl, C.A., Sklenar, J., Macho, A.P., Ntoukakis, V., Derbyshire, P., Altenbach, D., Maclean, D., Robatzek, S., Uhrig, J., Menke, F., Zhou, J.M., and Zipfel, C. 2016. The Arabidopsis protein phosphatase PP2C38 negatively regulates the central immune kinase BIK1. PLoS Pathog. 12:e1005811.

Cui, H., Xiang, T., and Zhou, J.M. 2009. Plant immunity: a lesson from pathogenic bacterial effector proteins. Cell Microbiol. 11:1453-1461.

Dodds, P.N., and Rathjen, J.P. 2010. Plant immunity: towards an integrated view of plant-pathogen interactions. Nat. Rev. Genet. 11:539-548.

Dong, J., Xiao, F., Fan, F., Gu, L., Cang, H., Martin, G.B., and Chai, J. 2009. Crystal structure of the complex between Pseudomonas effector AvrPtoB and the tomato Pto kinase reveals both a shared and a unique interface compared with AvrPto-Pto. Plant Cell 21:1846-1859.

Feng, F., and Zhou, J.M. 2012. Plant-bacterial pathogen interactions mediated by type III effectors. Curr. Opin. Plant Biol. 15:469-476. 
Feng, F., Yang, F., Rong, W., Wu, X., Zhang, J., Chen, S., He, C., and Zhou, J.M. 2012. A Xanthomonas uridine 5'-monophosphate transferase inhibits plant immune kinases. Nature 485:114-118.

Gabriëls, S.H.E.J., Vossen, J.H., Ekengren, S.K., van Ooijen, G., Abd-El-Haliem, A.M., van den Berg, G.C.M., Rainey, D.Y., Martin, G.B., Takken, F.L.W., de Wit, P.J.G.M., and Joosten, M.H.A.J. 2007. An NB-LRR protein required for HR signalling mediated by both extra- and intracellular resistance proteins. Plant J. 50:14-28.

Gao, M.H., Wang, X., Wang, D.M., Xu, F., Ding, X.J., Zhang, Z.B., Bi, D.L., Cheng, Y.T., Chen, S., Li, X., and Zhang, Y.L. 2009. Regulation of cell death and innate immunity by two receptor-like kinases in Arabidopsis. Cell Host Microbe 6:34-44.

Gimenez-lbanez, S., Hann, D.R., Ntoukakis, V., Petutschnig, E., Lipka, V., and Rathjen, J.P. 2009. AvrPtoB targets the LysM receptor kinase CERK1 to promote bacterial virulence on plants. Curr. Biol. 19:423429.

Gohre, V., Spallek, T., Haweker, H., Mersmann, S., Mentzel, T., Boller, T., de Torres, M., Mansfield, J.W., and Robatzek, S. 2008. Plant pattern-recognition receptor FLS2 is directed for degradation by the bacterial ubiquitin ligase AvrPtoB. Curr. Biol. 18:1824-1832.

Gomez-Gomez, L., and Boller, T. 2000. FLS2: an LRR receptor-like kinase involved in the perception of the bacterial elicitor flagellin in Arabidopsis. Mol. Cell 5:1003-1011.

Gravino, M., Locci, F., Tundo, S., Cervone, F., Savatin, D.V., and De Lorenzo, G. 2017. Immune responses induced by oligogalacturonides are differentially affected by AvrPto and loss of BAK1/BKK1 and PEPR1/PEPR2. Mol. Plant Pathol. 18:582-595.

Gust, A.A., and Felix, G. 2014. Receptor like proteins associate with SOBIR1-type of adaptors to form bimolecular receptor kinases. Curr. Opin. Plant Biol. 21:104-111.

He, P., Shan, L., Lin, N.C., Martin, G.B., Kemmerling, B., Nurnberger, T., and Sheen, J. 2006. Specific bacterial suppressors of MAMP signaling upstream of MAPKKK in Arabidopsis innate immunity. Cell 125:563575.

Hegenauer, V., Furst, U., Kaiser, B., Smoker, M., Zipfel, C., Felix, G., Stahl, M., and Albert, M. 2016. Detection of the plant parasite Cuscuta reflexa by a tomato cell surface receptor. Science 353:478-481.

Janeway, C.A., Jr. 1989. Approaching the asymptote? Evolution and revolution in immunology. Cold Spring Harb. Symp. Quant. Biol. 54 Pt 1:1-13.

Jehle, A.K., Furst, U., Lipschis, M., Albert, M., and Felix, G. 2013. Perception of the novel MAMP eMax from different Xanthomonas species requires the Arabidopsis receptor-like protein ReMAX and the receptor kinase SOBIR. Plant Signal. Behav. 8:e27408.

Jones, J.D., and Dangl, J.L. 2006. The plant immune system. Nature 444:323-329.

Kadota, Y., Shirasu, K., and Zipfel, C. 2015. Regulation of the NADPH oxidase RBOHD during plant immunity. Plant Cell Physiol. 56:1472-1480.

Kang, L., Tang, X., and Mysore, K.S. 2004. Pseudomonas Type III effector AvrPto suppresses the programmed cell death induced by two nonhost pathogens in Nicotiana benthamiana and tomato. Mol. Plant Microbe Interact. 17:1328-1336.

Kim, Y.J., Lin, N.C., and Martin, G.B. 2002. Two distinct Pseudomonas effector proteins interact with the Pto kinase and activate plant immunity. Cell 109:589-598.

Lee, A.H., Middleton, M.A., Guttman, D.S., and Desveaux, D. 2013. Phytopathogen type III effectors as probes of biological systems. Microb. Biotechnol. 6:230-240.

Lee, D., Bourdais, G., Yu, G., Robatzek, S., and Coaker, G. 2015. Phosphorylation of the plant immune regulator RPM1-INTERACTING PROTEIN4 enhances plant plasma membrane $\mathrm{H}^{+}$-ATPase activity and inhibits flagellin-triggered immune responses in Arabidopsis. Plant Cell 27:2042-2056.

Li, J., Wen, J., Lease, K.A., Doke, J.T., Tax, F.E., and Walker, J.C. 2002. BAK1, an Arabidopsis LRR receptor-like protein kinase, interacts with BRI1 and modulates brassinosteroid signaling. Cell 110:213-222.

Liebrand, T.W.H., van den Burg, H.A., and Joosten, M.H.A.J. 2014. Two for all: receptor-associated kinases SOBIR1 and BAK1. Trends Plant Sci. 19:123-132. 
Liebrand, T.W.H., Smit, P., Abd-El-Haliem, A.M., de Jonge, R., Cordewener, J.H.G., America, A.H.P., Sklenar, J., Jones, A.M.E., Robatzek, S., Thomma, B.P.H.J., Tameling, W.I.L., and Joosten, M.H.A.J. 2012. Endoplasmic reticulum-quality control chaperones facilitate the biogenesis of $\mathrm{Cf}$ receptor-like proteins involved in pathogen resistance of tomato. Plant Physiol. 159:1819-1833.

Liebrand, T.W.H., van den Berg, G.C.M., Zhang, Z., Smit, P., Cordewener, J.H.G., America, A.H.P., Sklenar, J., Jones, A.M.E., Tameling, W.I.L., Robatzek, S., Thomma, B.P.H.J., and Joosten, M.H.A.J. 2013. Receptorlike kinase SOBIR1/EVR interacts with receptor-like proteins in plant immunity against fungal infection. Proc. Natl. Acad. Sci. U.S.A. 110:10010-10015.

Lin, W., Li, B., Lu, D., Chen, S., Zhu, N., He, P., and Shan, L. 2014. Tyrosine phosphorylation of protein kinase complex BAK1/BIK1 mediates Arabidopsis innate immunity. Proc. Natl. Acad. Sci. U.S.A. 111:36323637.

Liu, Y., Huang, X., Li, M., He, P., and Zhang, Y. 2016. Loss-of-function of Arabidopsis receptor-like kinase BIR1 activates cell death and defense responses mediated by BAK1 and SOBIR1. New Phytol. 212:637-645.

Loh, Y.T., and Martin, G.B. 1995. The Pto bacterial resistance gene and the Fen insecticide sensitivity gene encode functional protein kinases with serine/threonine specificity. Plant Physiol. 108:1735-1739.

Lu, D., Wu, S., Gao, X., Zhang, Y., Shan, L., and He, P. 2010. A receptor-like cytoplasmic kinase, BIK1, associates with a flagellin receptor complex to initiate plant innate immunity. Proc. Natl. Acad. Sci. U.S.A. 107:496-501.

Ma, L.S., and Borhan, M.H. 2015. The receptor-like kinase SOBIR1 interacts with Brassica napus LepR3 and is required for Leptosphaeria maculans AvrLm1-triggered immunity. Front. Plant Sci. 6.

$\mathrm{Ma}, \mathrm{W}$. 2014. From pathogen recognition to plant immunity: BIK1 CROSses the divide. Cell Host Microbe 15:253-254.

Macho, A.P., and Zipfel, C. 2015. Targeting of plant pattern recognition receptor-triggered immunity by bacterial type-III secretion system effectors. Curr. Opin. Microbiol. 23:14-22.

Macho, A.P., Schwessinger, B., Ntoukakis, V., Brutus, A., Segonzac, C., Roy, S., Kadota, Y., Oh, M.H., Sklenar, J., Derbyshire, P., Lozano-Duran, R., Malinovsky, F.G., Monaghan, J., Menke, F.L., Huber, S.C., He, S.Y., and Zipfel, C. 2014. A bacterial tyrosine phosphatase inhibits plant pattern recognition receptor activation. Science 343:1509-1512.

Mackey, D., Holt, B.F., 3rd, Wiig, A., and Dangl, J.L. 2002. RIN4 interacts with Pseudomonas syringae type III effector molecules and is required for RPM1-mediated resistance in Arabidopsis. Cell 108:743-754.

Martin, G.B., Brommonschenkel, S.H., Chunwongse, J., Frary, A., Ganal, M.W., Spivey, R., Wu, T., Earle, E.D., and Tanksley, S.D. 1993. Map-based cloning of a protein kinase gene conferring disease resistance in tomato. Science 262:1432-1436.

Mucyn, T.S., Clemente, A., Andriotis, V.M., Balmuth, A.L., Oldroyd, G.E., Staskawicz, B.J., and Rathjen, J.P. 2006. The tomato NBARC-LRR protein Prf interacts with Pto kinase in vivo to regulate specific plant immunity. Plant Cell 18:2792-2806.

Pedley, K.F., and Martin, G.B. 2003. Molecular basis of Pto-mediated resistance to bacterial speck disease in tomato. Annu. Rev. Phytopathol. 41:215-243.

Peng, K.C., Wang, C.W., Wu, C.H., Huang, C.T., and Liou, R.F. 2015. Tomato SOBIR1/EVR homologs are involved in elicitin perception and plant defense against the oomycetepathogen Phytophthora parasitica. Mol. Plant Microbe Interact. 28:913-926.

Postma, J., Liebrand, T.W.H., Bi, G., Evrard, A., Bye, R.R., Mbengue, M., Kuhn, H., Joosten, M.H.A.J., and Robatzek, S. 2016. Avr4 promotes Cf-4 receptor-like protein association with the BAK1/SERK3 receptor-like kinase to initiate receptor endocytosis and plant immunity. New Phytol. 210:627-642.

Salmeron, J.M., Barker, S.J., Carland, F.M., Mehta, A.Y., and Staskawicz, B.J. 1994. Tomato mutants altered in bacterial disease resistance provide evidence for a new locus controlling pathogen recognition. Plant Cell 6:511-520.

Scofield, S.R., Tobias, C.M., Rathjen, J.P., Chang, J.H., Lavelle, D.T., Michelmore, R.W., and Staskawicz, B.J. 1996. Molecular basis of gene-for-gene specificity in bacterial speck disease of tomato. Science 274:2063-2065. 
Segonzac, C., Macho, A.P., Sanmartin, M., Ntoukakis, V., Sanchez-Serrano, J.J., and Zipfel, C. 2014. Negative control of BAK1 by protein phosphatase 2A during plant innate immunity. EMBO J. 33:2069-2079.

Shan, L., Thara, V.K., Martin, G.B., Zhou, J.M., and Tang, X. 2000. The Pseudomonas AvrPto protein is differentially recognized by tomato and tobacco and is localized to the plant plasma membrane. Plant Cell 12:2323-2338.

Shan, L., He, P., Li, J., Heese, A., Peck, S.C., Nurnberger, T., Martin, G.B., and Sheen, J. 2008. Bacterial effectors target the common signaling partner BAK1 to disrupt multiple MAMP receptor-signaling complexes and impede plant immunity. Cell Host Microbe 4:17-27.

Shao, F., Golstein, C., Ade, J., Stoutemyer, M., Dixon, J.E., and Innes, R.W. 2003. Cleavage of Arabidopsis PBS1 by a bacterial type III effector. Science 301:1230-1233.

Stulemeijer, I.J.E., Stratmann, J.W., and Joosten, M.H.A.J. 2007. Tomato mitogen-activated protein kinases LeMPK1, LeMPK2, and LeMPK3 are activated during the Cf-4/Avr4-induced hypersensitive response and have distinct phosphorylation specificities. Plant Physiol. 144:1481-1494.

Sun, Y., Li, L., Macho, A.P., Han, Z., Hu, Z., Zipfel, C., Zhou, J.M., and Chai, J. 2013. Structural basis for flg22induced activation of the Arabidopsis FLS2-BAK1 immune complex. Science 342:624-628.

Swiderski, M.R., and Innes, R.W. 2001. The Arabidopsis PBS1 resistance gene encodes a member of a novel protein kinase subfamily. Plant J. 26:101-112.

Takahashi, T., Shibuya, H., and Ishikawa, A. 2016. SOBIR1 contributes to non-host resistance to Magnaporthe oryzae in Arabidopsis. Biosci. Biotechnol. Biochem. 80:1577-1579.

Tang, D., Wang, G., and Zhou, J.M. 2017. Receptor kinases in plant-pathogen interactions: more than pattern recognition. Plant Cell 29:618-637.

Underwood, W., Zhang, S., and He, S.Y. 2007. The Pseudomonas syringae type III effector tyrosine phosphatase HopAO1 suppresses innate immunity in Arabidopsis thaliana. Plant J. 52:658-672.

Van der Hoorn, R.A., Laurent, F., Roth, R., and De Wit, P.J. 2000. Agroinfiltration is a versatile tool that facilitates comparative analyses of Avr9/Cf-9-induced and Avr4/Cf-4-induced necrosis. Mol. Plant Microbe Interact. 13:439-446.

Voinnet, O., Rivas, S., Mestre, P., and Baulcombe, D. 2015. An enhanced transient expression system in plants based on suppression of gene silencing by the p19 protein of tomato bushy stunt virus (Retration of vol 33, pg 949, 2003). Plant J. 84:846.

Wang, Y., Li, J., Hou, S., Wang, X., Li, Y., Ren, D., Chen, S., Tang, X., and Zhou, J.M. 2010. A Pseudomonas syringae ADP-ribosyltransferase inhibits Arabidopsis mitogen-activated protein kinase kinases. Plant Cell 22:2033-2044.

Warren, R.F., Henk, A., Mowery, P., Holub, E., and Innes, R.W. 1998. A mutation within the leucine-rich repeat domain of the Arabidopsis disease resistance gene RPS5 partially suppresses multiple bacterial and downy mildew resistance genes. Plant Cell 10:1439-1452.

Wu, S., Lu, D., Kabbage, M., Wei, H.L., Swingle, B., Records, A.R., Dickman, M., He, P., and Shan, L. 2011. Bacterial effector HopF2 suppresses Arabidopsis innate immunity at the plasma membrane. Mol. Plant Microbe Interact. 24:585-593.

Xiang, T., Zong, N., Zhang, J., Chen, J., Chen, M., and Zhou, J.M. 2011. BAK1 is not a target of the Pseudomonas syringae effector AvrPto. Mol. Plant Microbe Interact. 24:100-107.

Xiang, T., Zong, N., Zou, Y., Wu, Y., Zhang, J., Xing, W., Li, Y., Tang, X., Zhu, L., Chai, J., and Zhou, J.M. 2008. Pseudomonas syringae effector AvrPto blocks innate immunity by targeting receptor kinases. Curr. Biol. 18:74-80.

Xing, W., Zou, Y., Liu, Q., Liu, J., Luo, X., Huang, Q., Chen, S., Zhu, L., Bi, R., Hao, Q., Wu, J.W., Zhou, J.M., and Chai, J. 2007. The structural basis for activation of plant immunity by bacterial effector protein AvrPto. Nature 449:243-247.

Xu, J., Xie, J., Yan, C., Zou, X., Ren, D., and Zhang, S. 2014. A chemical genetic approach demonstrates that MPK3/MPK6 activation and NADPH oxidase-mediated oxidative burst are two independent signaling events in plant immunity. Plant J. 77:222-234. 
Yasuda, S., Okada, K., and Saijo, Y. 2017. A look at plant immunity through the window of the multitasking coreceptor BAK1. Curr. Opin. Plant Biol. 38:10-18.

Zeng, L., Velasquez, A.C., Munkvold, K.R., Zhang, J., and Martin, G.B. 2012. A tomato LysM receptor-like kinase promotes immunity and its kinase activity is inhibited by AvrPtoB. Plant J. 69:92-103.

Zhang, J., Shao, F., Li, Y., Cui, H., Chen, L., Li, H., Zou, Y., Long, C., Lan, L., Chai, J., Chen, S., Tang, X., and Zhou, J.M. 2007. A Pseudomonas syringae effector inactivates MAPKs to suppress PAMP-induced immunity in plants. Cell Host Microbe 1:175-185.

Zhang, J., Li, W., Xiang, T., Liu, Z., Laluk, K., Ding, X., Zou, Y., Gao, M., Zhang, X., Chen, S., Mengiste, T., Zhang, Y., and Zhou, J.M. 2010. Receptor-like cytoplasmic kinases integrate signaling from multiple plant immune receptors and are targeted by a Pseudomonas syringae effector. Cell Host Microbe 7:290-301.

Zhang, L., Kars, I., Essenstam, B., Liebrand, T.W.H., Wagemakers, L., Elberse, J., Tagkalaki, P., Tjoitang, D., van den Ackerveken, G., and van Kan, J.A.L. 2014. Fungal endopolygalacturonases are recognized as microbe-associated molecular patterns by the Arabidopsis receptor-like protein RESPONSIVENESS TO BOTRYTIS POLYGALACTURONASES1. Plant Physiol. 164:352-364.

Zhang, W.G., Fraiture, M., Kolb, D., Loffelhardt, B., Desaki, Y., Boutrot, F.F.G., Tor, M., Zipfel, C., Gust, A.A., and Brunner, F. 2013a. Arabidopsis receptor-like protein30 and receptor-like kinase suppressor of BIR1-1/EVERSHED mediate innate immunity to necrotrophic fungi. Plant Cell 25:4227-4241.

Zhang, Z., Fradin, E., de Jonge, R., van Esse, H.P., Smit, P., Liu, C.M., and Thomma, B.P.H.J. 2013b. Optimized agroinfiltration and virus-induced gene silencing to study Ve1-mediated Verticillium resistance in tobacco. Mol. Plant Microbe Interact. 26:182-190.

Zhang, Z., Wu, Y., Gao, M., Zhang, J., Kong, Q., Liu, Y., Ba, H., Zhou, J., and Zhang, Y. 2012. Disruption of PAMP-induced MAP kinase cascade by a Pseudomonas syringae effector activates plant immunity mediated by the NB-LRR protein SUMM2. Cell Host Microbe 11:253-263.

Zhou, J., Wu, S., Chen, X., Liu, C., Sheen, J., Shan, L., and He, P. 2014. The Pseudomonas syringae effector HopF2 suppresses Arabidopsis immunity by targeting BAK1. Plant J. 77:235-245.

Zipfel, C. 2014. Plant pattern-recognition receptors. Trends Immunol. 35:345-351.

Zipfel, C., Kunze, G., Chinchilla, D., Caniard, A., Jones, J.D., Boller, T., and Felix, G. 2006. Perception of the bacterial PAMP EF-Tu by the receptor EFR restricts Agrobacterium-mediated transformation. Cell 125:749-760.

Zipfel C, Robatzek S, Navarro L, Oakeley EJ, Jones JD, Felix G, Boller T. 2004. Bacterial disease resistance in Arabidopsis through flagellin perception. Nature 428: 764. 


\section{Supplemental data}
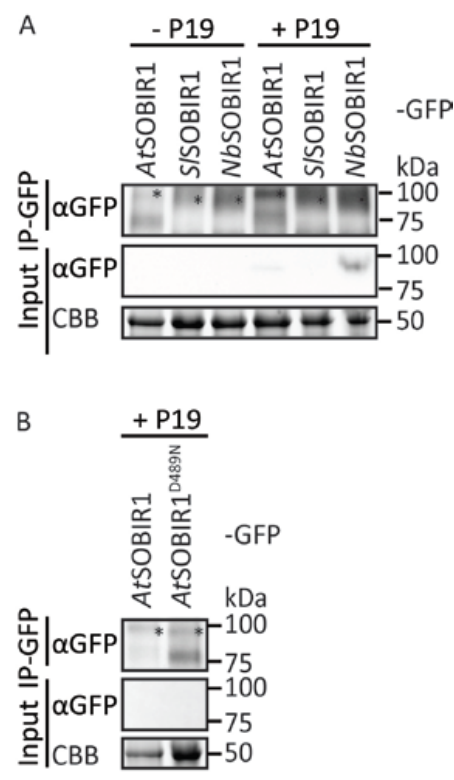

Figure S1. All SOBIR1 variants accumulate in planta. (A) Accumulation levels of At-, Sl- and NbSOBIR1 without and with silencing suppressor P19. C-terminally eGFP-tagged SOBIR1 variants were transiently expressed in $N$. benthamiana with an $O D$ of 1 . After 2 days, total proteins were extracted and subjected to immunoprecipitation using GFP_TrapA affinity beads. Immunoprecipitated proteins (IP) were subjected to SDSPAGE and blotted to PVDF membrane. Blots were probed with aGFP antibody to detect eGFP-tagged proteins (upper panels). Total protein staining indicates the amount of protein that was loaded (lower panel). Bands with the expected size of fulllength AtSOBIR1-eGFP, SISOBIR1-eGFP and NbSOBIR1-eGFP are indicated with an asterisk. (B) Accumulation levels of AtSOBIR1 and AtSOBIR1 ${ }^{D 489 N}$. The experiment was performed as described for panel A. 


\section{Chapter 3}

\section{Transphosphorylation between SOBIR1 and BAK1 is required for immune signalling}

Aranka M. van der Burgh'1, Jelle Postma², Silke Robatzek², Matthieu H. A. J. Joosten ${ }^{1}$

${ }^{1}$ Laboratory of Phytopathology, Wageningen University, Droevendaalsesteeg 1, 6708 PB Wageningen, The Netherlands

${ }^{2}$ The Sainsbury Laboratory, Norwich Research Park, Norwich NR4 7UH, UK 


\section{Abstract}

Leucine-rich repeat-receptor-like proteins (LRR-RLPs) and LRR-receptor-like kinases (LRR-RLKs) trigger immune signalling to promote plant resistance against pathogens. LRR-RLPs lack an intracellular kinase domain, and they constitutively interact with the LRR-RLK Suppressor Of BIR1-1/Evershed (SOBIR1/EVR) to form signalling-competent receptor complexes. Ligand perception by LRR-RLPs causes the recruitment of the co-receptor BRI1-Associated Kinase 1/Somatic Embryogenesis Receptor Kinase 3 (BAK1/SERK3) to the LRR-RLP/SOBIR1 complex, thereby activating LRR-RLP-mediated immunity. We employed phosphorylation analysis of in planta produced proteins, live-cell imaging, gene silencing, and co-immunoprecipitation to investigate the roles of SOBIR1 and BAK1 in immune signalling. We show that Arabidopsis thaliana (At) SOBIR1, which constitutively activates immune responses upon its overexpression in planta, is highly phosphorylated. Moreover, apart from kinase activity of SOBIR1, kinase-active BAK1 is essential for AtSOBIR1-induced constitutive immunity and for the phosphorylation of AtSOBIR1. Furthermore, the defence response triggered upon perception of Avr4 from the extracellular pathogenic fungus Cladosporium fulvum by the tomato LRR-RLP Cf-4, depends on kinase-active BAK1. We propose that, besides trans-autophosphorylation of SOBIR1, it is likely that SOBIR1 and BAK1 transphosphorylate, and thereby activate the receptor complex. The signallingcompetent cell surface receptor complex subsequently activates downstream cytoplasmic signalling partners to initiate RLP-mediated immunity. 


\section{Introduction}

Plants are sessile organisms and have several layers of defence to protect against pathogens. One layer comprises transmembrane (TM)-receptors that are present at the cell surface. Unlike mammals, plants have evolved highly expanded families of TM-receptors, which sense non-self or danger-related molecules in the extracellular space and initiate downstream signalling to mount plant immunity (Zipfel, 2014). TMreceptors comprise receptor-like kinases (RLKs) and receptor-like proteins (RLPs). Both RLKs and RLPs contain ectodomains for ligand recognition, which in many cases are leucine-rich repeat (LRR) domains (Böhm et al., 2014; Macho \& Zipfel, 2014; Couto \& Zipfel, 2016). LRR-RLKs (further referred to as RLKs) carry an intracellular kinase domain, whereas LRR-RLPs (further referred to as RLPS) do not. Whilst expansion of receptor families enables co-evolution with infectious pathogens, it challenges plants to link newly evolved receptors with downstream immune signalling pathways. In line with this, it is now emerging that receptors form heteromeric kinase complexes to induce immune signalling (Fischer et al., 2016; Dufayard et al., 2017).

RLPs, lacking an intracellular signalling domain, constitutively interact with the RLK Suppressor Of BIR1-1/Evershed (SOBIR1/EVR, hereafter referred to as SOBIR1), thereby providing a kinase domain that is thought to function in downstream signalling (Gao et al., 2009; Leslie et al., 2010; Liebrand et al., 2014; Gust \& Felix, 2014). SOBIR1 was initially found to interact with Cf proteins from tomato (Solanum lycopersicum, Sl), conferring resistance to the extracellular fungal pathogen Cladosporium fulvum (Liebrand et al., 2013). Since this initial discovery, it has been shown that SOBIR1 constitutively interacts with many RLPs involved in immunity and development (Liebrand et al., 2013; Hegenauer et al., 2016; Catanzariti et al., 2017; Domazakis et al., 2018; Ma \& Borhan, 2015; Wang et al., 2018; Jehle et al., 2013; Bi et al., 2014; Zhang et al., 2013 and 2014). In addition to stabilisation of the associated RLP by SOBIR1, SOBIR1 is thought to be involved in downstream signalling upon RLP activation by its matching ligand (Liebrand et al., 2013 and 2014).

The RLK BRI1-Associated Receptor Kinase 1/Somatic Embryogenesis Receptor Kinase 3 (BAK1/SERK3, hereafter referred to as BAK1) functions as a co-receptor of several well-studied RLKs from Arabidopsis, including Flagellin-Sensing 2 (FLS2), Elongation Factor-Tu Receptor (EFR), and brassinosteroid (BR) receptor Brassinosteroid-Insensitive 1 (BRI1) (Zipfel et al., 2006; Gómez-Gómez \& Boller, 2000; Li \& Chory, 1997; Nam \& Li, 2002). These RLKs form complexes with BAK1 upon association with their ligands (Chinchilla et al., 2007; Heese et al., 2007; Somssich et al., 2015; Sun et al., 2013a and 2013b; Santiago et al., 2013). RLK/BAK1-complex formation is followed by transphosphorylation of both kinase domains, the initiation of downstream signalling, and internalization of the activated RLK/BAK1 complex through endocytosis (Schwessinger et al., 2011; Wang et al., 2008; Frescatada-Rosa 
et al., 2015; Couto \& Zipfel, 2016). Differential auto- and transphosphorylation is suggested to take place for BAK1 in complex with RLKs either signalling for defence or development, and this differential phosphorylation eventually leads to the desired output (Oh et al., 2011; Schwessinger et al., 2011; Wang et al., 2008; Macho et al., 2014; Macho et al., 2015).

Similar to the situation with RLKs, BAK1 was recently also found to be recruited to two-component RLP/SOBIR1-complexes upon ligand recognition by the RLP involved (Albert et al., 2015; Postma et al., 2016; Wang et al., 2018; Domazakis et al., 2018). For example, BAK 1 is specifically recruited to the Cf-4/SOBIR1 complex upon perception of Avr4 from C. fulvum by Cf-4 (Postma et al., 2016). Similarly, Albert and co-workers (2015) showed that the RLP23/SOBIR1 complex recruits BAK1 to mediate immunity triggered by necrosis and ethylene-inducing peptide-like 1 proteins (NLPs). Reminiscent of BAK1-mediated receptor complex activation for cell surface complexes involving the RLKS FLS2, EFR, and BRI1, it is likely that BAK1, upon its recruitment, also forms signalling-competent receptor complexes with RLP/SOBIR1 bi-partite RLKs, thereby mediating RLP signalling. In agreement with this, SOBIR1 kinase activity is essential for its function downstream of $\mathrm{Cf}-4$, as it has been shown that a kinase-dead mutant of SOBIR 1 is unable to complement in Cf-4/Avr4 signalling and in endocytosis of the Cf-4/SOBIR1 complex (Liebrand et al., 2013; Bi et al., 2015; Postma et al., 2016). However, up till now, it has remained unclear how the kinase domain of SOBIR1 exactly contributes to RLP/SOBIR1 receptor complex signalling.

SOBIR1 was initially identified as a positive regulator of cell death. It was found that Arabidopsis BAK1-Interacting RLK 1 (bir1-1) loss-of-function mutants showed severe dwarfing as a result of constitutive immunity, which was partially suppressed by a sobir 1 loss-of-function mutation (Gao et al., 2009). Furthermore, overexpression of SOBIR1 in Arabidopsis induced constitutive immunity, which was observed as constitutive cell death and defence gene activation (Gao et al., 2009). BIR1 is a negative regulator of defence, which sequesters BAK1 away from active signalling complexes (Gao et al., 2009; Liu et al., 2016). Liu and co-workers showed that upon silencing of BIR1 in Arabidopsis, more BAK1 becomes available, and constitutively interacts with SOBIR1 (Liu et al., 2016).

Recently, we observed that transient heterologous overexpression of AtSOBIR1 in Nicotiana tabacum (tobacco, $N$ t) and $N$. benthamiana $(\mathrm{Nb})$, in contrast to transient overexpression of SISOBIR1 and NbSOBIR1, also results in constitutive immunity (Wu et al., 2018). This constitutive immunity typically manifests itself by the induction of cell death (the hypersensitive response (HR)) and mitogen-activated protein kinase (MAPK) activation (Wu et al., 2018). Kinase-dead AtSOBIR1 $1^{\text {D498N }}$ does not induce constitutive immunity, indicating that SOBIR1 kinase activity is essential for this phenomenon. The lack of constitutive immune activation by SISOBIR1 and NbSOBIR1 in tobacco and $N$. benthamiana might be explained by proper negative regulation 
of these Solanaceous orthologues of SOBIR1 by endogenous phosphatases of the Solanaceous plants tobacco and $N$. benthamiana. Negative regulation of immune receptors via dephosphorylation is a well-known phenomenon (Couto \& Zipfel, 2016). BAK1, for example, has been shown to be negatively regulated by Protein Phosphatase type 2A (PP2A) (Segonzac et al., 2014). Possibly, endogenous Solanaceous phosphatases successfully negatively regulate the activity of tomato and $N$. benthamiana SOBIR1 via dephosphorylation. However, these phosphatases might not be able to keep AtSOBIR1 in check, for example due to lower affinity for this heterologous SOBIR1 orthologue (Wu et al., 2018).

Here, we set out to determine how SOBIR1 activates defence signalling. For this, we exploited the phenomenon of AtSOBIR1 constitutive immune signalling in $N$. benthamiana, leading to cell death. We show that AtSOBIR1 is highly phosphorylated when transiently expressed in $N$. benthamiana, and that the level of overall AtSOBIR1 phosphorylation is positively correlated with its constitutive immune activity. We found that SOBIR1 is able to constitutively form homodimers, as well as heterodimers with BAK1. Interestingly, the $\mathrm{Cf}$-4/Avr4-triggered defence response, as well as AtSOBIR1-constitutive immunity and AtSOBIR1 phosphorylation, all depend on defence signalling-competent BAK1. These findings are in agreement with a model in which, upon ligand perception by an RLP, BAK1 is recruited to the RLP/SOBIR1 complex, after which BAK1 and SOBIR1 transphosphorylate each other to signal for immunity.

\section{Results}

\section{Constitutive immune activity of AtSOBIR1 is positively correlated with its phosphorylation}

Leslie and co-workers (2010) showed that the kinase domain of AtSOBIR1 transautophosphorylates in vitro, at Ser, Thr, and Tyr residues. To determine whether in planta phosphorylation of SOBIR1 plays a role in signalling for constitutive immunity induced by this RLK (Wu et al., 2018), we transiently overexpressed full-length eGFPtagged Arabidopsis and tomato SOBIR1 proteins and the corresponding kinase-dead mutants, in which the catalytic aspartic acid (Asp, D) is mutated to asparagine (Asn, $N$ ), in $N$. benthamiana, in combination with P19. Subsequently, we analysed their overall phosphorylation status by Pro-Q staining. This revealed that AtSOBIR1 is highly phosphorylated, whereas SISOBIR1 and both kinase-dead mutants are not (Fig. 1A). It appears that the phosphorylation status of the different SOBIR1 variants is positively correlated with their constitutive immune activity (Fig. S1A) (Wu et al., 2018). Lack of a Pro-Q signal for kinase-dead AtSOBIR1 indicates that wild-type AtSOBIR1 trans-autophosphorylates in planta. In addition, the low phosphorylation 
status of AtSOBIR1 ${ }^{\text {D489N }}$ suggests that it might be necessary for AtSOBIR1 to first activate a potential signalling partner, which then in its turn fully activates AtSOBIR1 by transphosphorylation. Interestingly, constitutive immunity, as well as functionality of SOBIR1 downstream of Cf-4, is dependent on SOBIR1-kinase activity (Liebrand et al., 2013; Wu et al., 2018). The stabilization of Cf-4 by SOBIR1 is independent of SOBIR1-kinase activity, so this stabilization effect by itself cannot explain the role of SOBIR1 in RLP-mediated signalling (Fig. S1B) (Liebrand et al., 2013). This again points to a signalling role by the kinase domain of SOBIR1. The lack of a Pro-Q signal for SISOBIR1 supports our hypothesis that proper negative regulation takes place through dephosphorylation of this Solanaceous SOBIR1 by endogenous phosphatases in N. benthamiana (Fig. 1A) (Wu et al., 2018; Couto \& Zipfel, 2016).

(A)

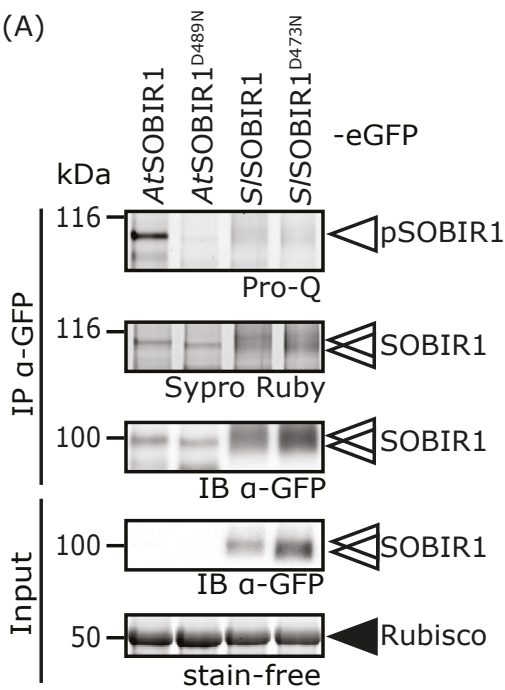

(B)
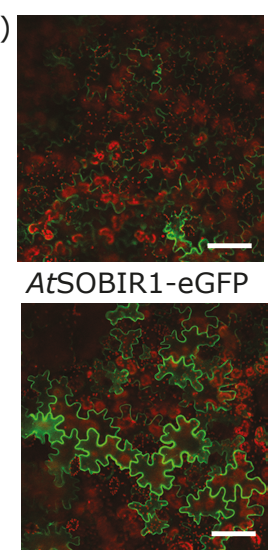

AtSOBIR1 $1^{\text {D489N-eGFP }}$

Figure 1. Constitutive immune activity of AtSOBIR1 positively correlates with its increased level of phosphorylation. (A) Pro-Q phosphoprotein staining of immunoprecipitated eGFP-tagged SOBIR1 variants from Arabidopsis and tomato shows constitutive phosphorylation of AtSOBIR1 (pAtSOBIR1), but not of SISOBIR1. Furthermore, both the Arabidopsis and tomato kinase-dead mutants show a very low level of phosphorylation. Agroinfiltrations were performed on $N$. benthamiana leaves at an $\mathrm{OD}_{600}$ of 1 , with co-infiltration of P19 also at an $\mathrm{OD}_{600}$ of 1 . Leaves were harvested about 40 hours after agroinfiltration, before cell death became apparent, followed by immunoprecipitation (IP) using anti-GFP affinity beads. Sypro Ruby staining and immune blotting (IB) show SOBIR1 protein levels. The Rubisco band of the input shows equal loading. See also Fig. S1. (B) AtSOBIR1 and its kinase-dead version localize at the plasma membrane. Note that AtSOBIR1 ${ }^{\mathrm{D} 489 \mathrm{~N}}$ accumulates to higher levels than wild-type AtSOBIR1. Leaves of tobacco were agroinfiltrated with constructs driving expression of eGFP-tagged wild-type AtSOBIR1 or the kinase-dead mutant AtSOBIR1 ${ }^{\text {D489N }}$, and analysed for their localization at 2 days post infiltration (dpi) using confocal microscopy. Chloroplast autofluorescence is depicted in red. White bars represent $100 \mu \mathrm{m}$. The experiment was performed three times and representative pictures are shown. 
Next to regulation of the signalling activity of immune receptors through their phosphorylation status, endocytosis followed by degradation is a well-known mechanism to negatively regulate immune receptors (Frescatada-Rosa et al., 2015; Couto \& Zipfel, 2016). To test whether AtSOBIR1 protein accumulation is affected by its constitutive activity, we expressed eGFP-tagged wild-type and kinase-dead AtSOBIR1 in tobacco leaves. Observation by confocal microscopy showed that both proteins localize at the plasma membrane (PM), but AtSOBIR1 ${ }^{\text {D489N }}$ accumulates to higher levels than wild-type AtSOBIR1 (Fig. 1B). Constitutively active AtSOBIR1 only accumulates to low levels, probably because continuous endocytosis and degradation of this autoactive regulatory RLK will take place (Wu et al., 2018). This difference in accumulation can also be observed on immunoblots (IB) presented by Wu and co-workers (2018). Lower accumulation of constitutively active AtSOBIR1 was not apparent in the IP experiment shown in Fig. 1A, likely due to the use of silencing suppressor P19, which probably increases early protein accumulation levels, before cell death occurs.

Together, these data show that constitutive immune activity of AtSOBIR 1 is positively correlated with its overall phosphorylation level, and that SISOBIR1 is kept in check by maintaining phosphorylation levels low.

\section{SOBIR1 constitutively forms homodimers}

To explore whether trans-autophosphorylation of SOBIR1 might play a role in signalling for defence by SOBIR1, we analysed whether SOBIR1 forms homodimers in planta. Transient co-expression of eGFP- and Myc-tagged SOBIR1 orthologues, followed by immunoprecipitation of eGFP-tagged SOBIR1, resulted in the co-purification of SOBIR1Myc (Fig. 2A). This indicates that both AtSOBIR1 and SISOBIR1 form homodimers in planta. Much higher amounts of SOBIR1-Myc co-purified with the pull down of Cf-4eGFP, here used as a positive control, than with the pull down of SOBIR1-eGFP (Fig. $2 A)$. This indicates that only a small pool of SOBIR1 proteins is present in the form of homodimers in planta, and this probably explains why homodimerization of SOBIR1 was not observed before (Liebrand et al., 2013). Similar to the earlier observation by Liebrand et al. (2013), SOBIR1-Myc does not co-purify with FLS2-eGFP, here used as negative control (Fig. 2A).

A split-YFP experiment also revealed homodimerization of SISOBIR1 in planta (Fig. 2B). For this, SISOBIR1 fused to the N-terminal half of YFP (nYFP) was co-expressed with SISOBIR1 fused to the C-terminal half of YFP (cYFP). Subsequent observation by confocal microscopy revealed a clear signal of reconstituted full-length YFP. As a positive control, co-expression of SISOBIR1-nYFP with Cf-4-cYFP was taken along, which also resulted in a clear YFP signal. Expression of SISOBIR1-nYFP in combination with AtFLS2-cYFP, taken along as a negative control, indeed did not reconstitute a YFP signal (Fig. 2B). A clear YFP signal upon co-expression of AtFLS2-cYFP and AtFLS2-nYFP (Fig. 2B) confirmed that also FLS2 forms homodimers in planta (Sun et al., 2012). 
As AtSOBIR1 is phosphorylated in planta (Fig. 1A), and is able to constitutively form homodimers in planta, it is mechanistically possible that SOBIR1 transautophosphorylates.
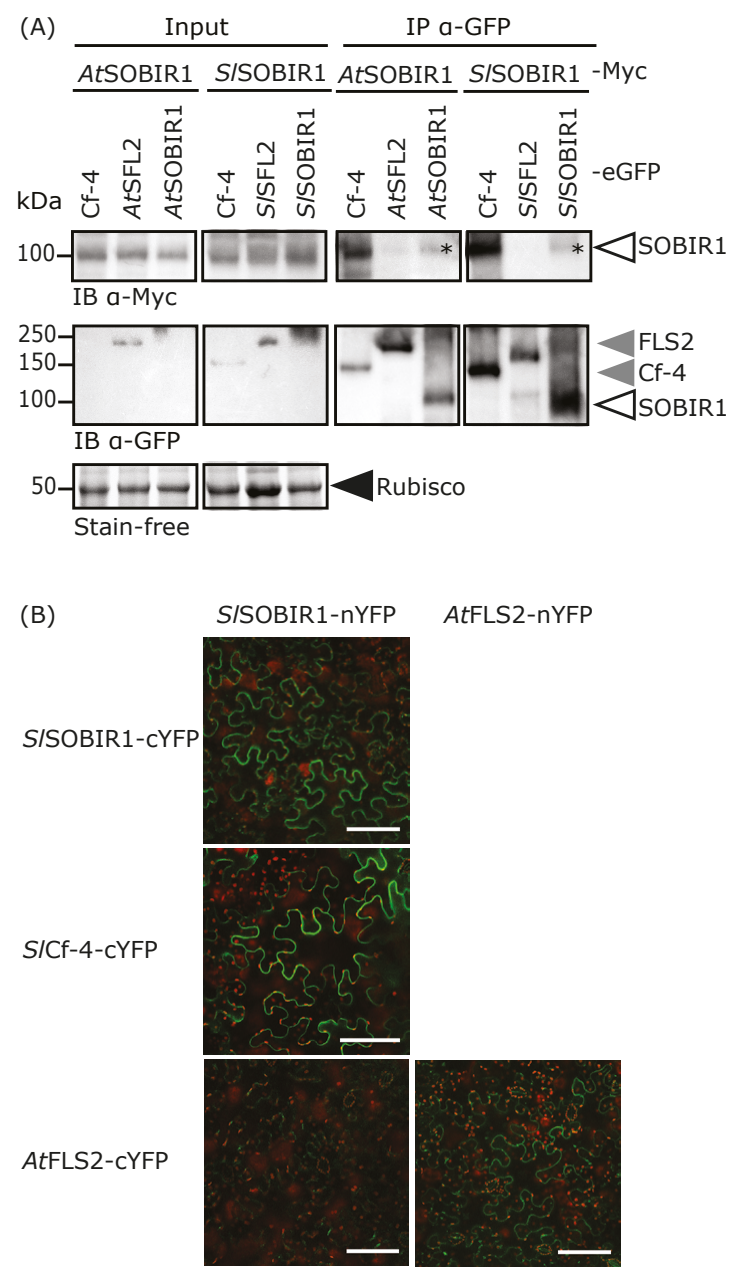

Figure 2. SOBIR1 constitutively forms homodimers in planta. (A) Myc-tagged versions of AtSOBIR1 and SISOBIR1 co-immunoprecipitate with eGFP-tagged versions of AtSOBIR1 and SISOBIR1 (asterisks), respectively, and with Cf-4-eGFP, but not with FLS2-eGFP. Co-agroinfiltrations of the various affinitytagged proteins were performed in combination with P19 in N. benthamiana leaves at an $\mathrm{OD}_{600}$ of 0.6 for each construct. Leaves were harvested at $2 \mathrm{dpi}$, and subjected to IP using anti-GFP beads, followed by IB. The Rubisco band of the input shows equal loading. The experiment was performed twice and representative results are shown. (B) Split-YFP shows interaction between SISOBIR1-nYFP and SISOBIR1CYFP at the plasma membrane. Leaves of $N$. benthamiana were agroinfiltrated with constructs driving expression of the indicated constructs, and analysed for interaction at 2 dpi using confocal microscopy. Chloroplast autofluorescence is depicted in red. White bars represent $100 \mu \mathrm{m}$. The experiment was performed three times and representative pictures are shown. 


\section{Constitutive immune activity of AtSOBIR1 is BAK1-dependent}

To analyse whether BAK1, next to its role in RLP/SOBIR1-mediated immunity, also plays a role in AtSOBIR1-induced constitutive immunity and phosphorylation of SOBIR1, we examined whether, besides ligand-induced BAK1 recruitment, also a constitutive interaction between BAK1 and SOBIR1 takes place. For that reason, we co-expressed tagged BAK1 and SOBIR1 in N. benthamiana in the presence of P19. We harvested leaves one day after agroinfiltration to avoid the occurrence of AtSOBIR1induced cell death, which would become apparent at two dpi. Interestingly, we found that BAK1 co-immunoprecipitates with AtSOBIR1 ${ }^{\text {D489N }}$ and SISOBIR1 (Fig. 3A). Wildtype AtSOBIR1 only accumulates to very low levels due to its constitutive immune activity (Wu et al., 2018; Gao et al., 2009), and therefore the presence of a possible interaction between AtBAK1 and AtSOBIR1 could not be determined (Fig. 3A). That we could not determine an interaction between AtBAK1 and AtSOBIR1 is in agreement with the findings of Liu and co-workers (2016), who showed an interaction between AtSOBIR1 and AtBAK1 only upon silencing of BIR 1 in Arabidopsis. Likely, a small pool of SOBIR1 and BAK1 constitutively interact when overexpressed in planta, and this pool becomes larger upon ligand elicitation (Albert et al., 2015; Postma et al.,2016; Wang et al., 2018), or BIR1 silencing (Liu et al., 2016). Together, these results indicate that SOBIR1 forms heterodimers with BAK1, independent of constitutive immune activity of SOBIR1, and the elevated phosphorylation status of constitutively-active AtSOBIR1 cannot simply be explained by SOBIR1 interaction with BAK1, as SISOBIR1 phosphorylation levels are not apparent (Fig. 1A).

To further address whether BAK1 is required for AtSOBIR1 constitutive immune activity, we employed virus-induced gene silencing (VIGS) to knockdown the expression of the AtBAK 1 homologues SERK3a/b and SERK1 in tobacco and $N$. benthamiana (Heese et al., 2007, Postma et al., 2016). For this, the VIGS construct pTRV2:NbSERK3a/b (Heese et al., 2007) was agro-inoculated onto tobacco (cv. Samsun, Zhang et al., 2013b), alongside with pTRV2:GUS as a negative control. Three weeks after agro-inoculation, plants exhibited the characteristic stunting phenotype due to silencing of the BAK1 homologues (not shown), and leaf sectors were transiently transformed to overexpress AtSOBIR1. Constitutive immune activity of AtSOBIR1 was highly compromised in plants inoculated with pTRV2:NbSERK3a/b, but not in the pTRV2:GUS-inoculated tobacco plants (Fig. 3B). A similar experiment was performed in N. benthamiana:Cf-4 (Gabriëls et al., 2006), for which inoculation with pTRV2:NbSERK3a/b also resulted in severe stunting which confirmed BAK 1 silencing (not shown), and suppressed AtSOBIR1 constitutive immune activity (Fig. 3B). Compromised Cf-4-mediated HR upon expression of Avr4 in these plants confirmed successful knockdown of $N b S E R K 3 a / b$, as these BAK1 homologues have been shown before to be required for the Cf-4/Avr4-triggered HR (Postma et al., 2016). 

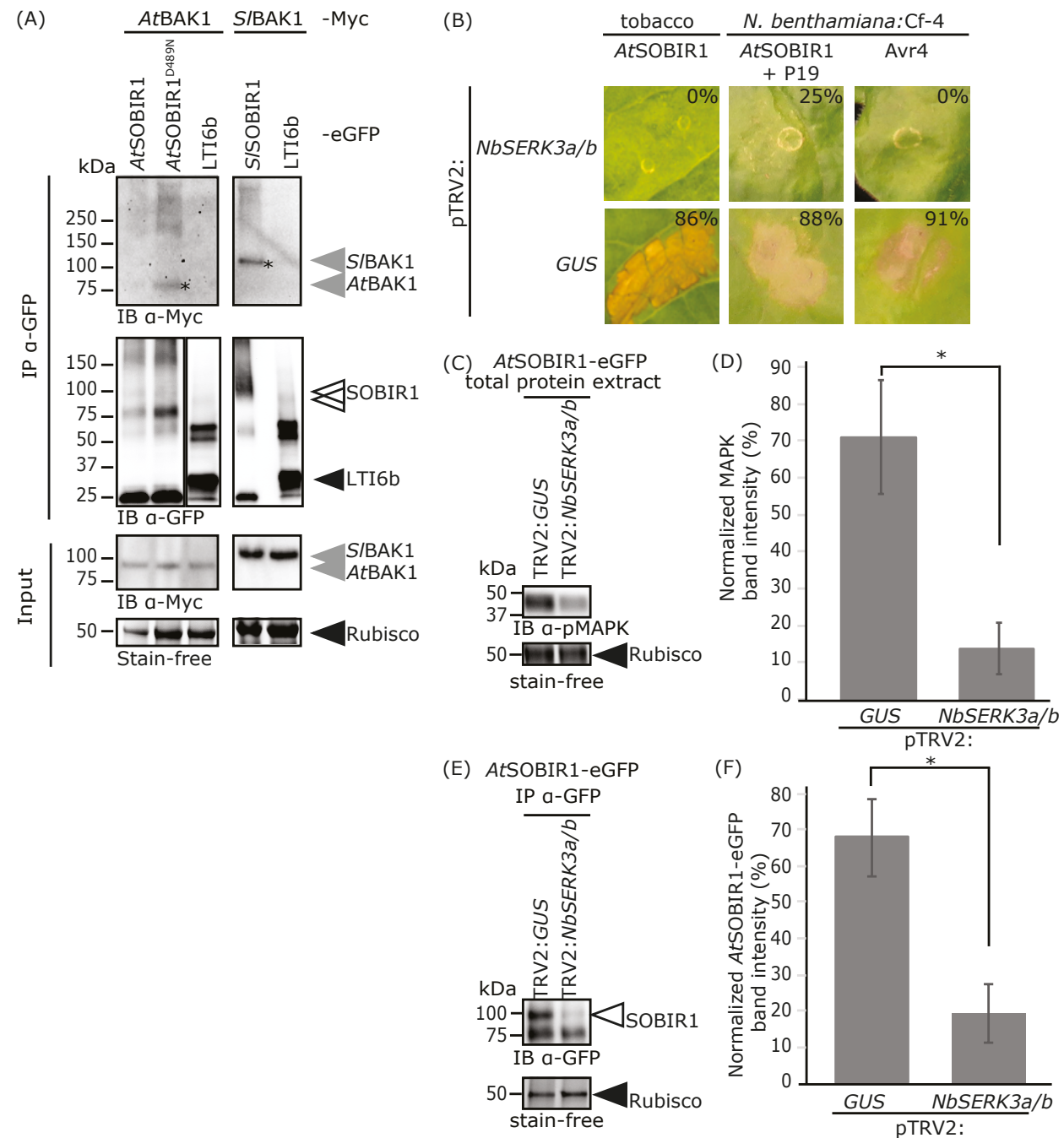

Figure 3. Constitutive immune activity of AtSOBIR1 is BAK1-dependent. (A) SOBIR1 constitutively interacts with BAK1. eGFP-tagged versions of SOBIR1 and Myc-tagged versions of BAK1 were transiently co-expressed in $N$. benthamiana at an OD of 0.6 for each construct. Leaves were harvested at $1 \mathrm{dpi}$, followed by IP and protein detection on IB. Note that the anti-Myc IB, revealing the co-IP of BAK1-Myc (asterisks), shows relatively faint signals, which suggests that only a small pool of the total amount of SOBIR1 and BAK1 proteins is constitutively interacting. The plasma membrane (PM) protein LOW TEMPERATURE-INDUCED 6b (LTI6b) (Kurup et al., 2005) was included as a negative control. Representative results of three independent experiments are shown. (B) Inoculation of tobacco and $N$. benthamiana:Cf-4 with pTRV2:NbSERK3a/b, leads to compromised constitutive immune activity of AtSOBIR1. AtSOBIR1-eGFP was transiently expressed by agroinfiltration at an $\mathrm{OD}_{600}$ of 1 , in pTRV2:GUS- or pTRV2:NbSERK3a/b-inoculated tobacco or Nb:Cf-4. In $\mathrm{Nb}: \mathrm{Cf}-4$, AtSOBIR1 was co-expressed with P19 at an $\mathrm{OD}_{600}$ of 1 . Transient expression of Avr4 at $\mathrm{OD}_{600} 0.03$ shows a compromised HR in the pTRV2:NbSERK3a/b-inoculated Nb:Cf-4 plants. Percentages of cell death were scored as described in Materials \& Methods. The experiments were performed at least three times, with testing at least three individual leaves per sample. Representative pictures, taken at $3 \mathrm{dpi}$, are shown. (C) Inoculation with pTRV2:NbSERK3a/b leads to reduced MAPK activation upon transient overexpression of 
AtSOBIR1. AtSOBIR1-eGFP was transiently co-expressed with $\mathrm{P} 19$, both at an $\mathrm{OD}_{600}$ of 1 , in $N$. benthamiana plants that were previously inoculated with the indicated TRV recombinants. About $40 \mathrm{~h}$ after agroinfiltration, total protein was extracted and analysed for MAPK activation using anti-p42/p44-erk antibody. The Rubisco band shows equal loading. The experiment was performed three times, and representative results are shown. (D) Quantification of the results of the experiment shown in B. Ratios were obtained by dividing the band intensity of phosphorylated MAPK by the intensity of the Rubisco input band. Data are represented as mean $+/$ - standard error (SE). The asterisk indicates significant difference $(P<0.05)$, as determined by a student's t-test. (E) Inoculation with pTRV2:NbSERK3a/b leads to reduced accumulation of AtSOBIR1-eGFP. AtSOBIR1-eGFP was transiently co-expressed with P19, both at an $\mathrm{OD}_{600}$ of 1 , in $N$. benthamiana plants that were previously inoculated with the indicated TRV recombinants. About $40 \mathrm{~h}$ after agroinfiltration, total protein was extracted and subjected to IP, followed by protein detection on IB. The Rubisco backgroundband of the IP samples is depicted to show equal loading. The experiment was performed three times, and representative results are shown. $(F)$ Quantification of the results of the experiment shown in D. Ratios were obtained by dividing the band intensity of AtSOBIR1-eGFP by the band intensity of the rubisco background in the IP samples. Data are represented as mean +/- SE. The asterisk indicates significant difference $(P<0.05)$, as determined by a student's t-test.

To confirm that the compromised cell death response, observed upon overexpression of AtSOBIR1 in N. benthamiana inoculated with pTRV2:NbSERK3a/b, is a consequence of reduced constitutive immune activity, we examined MAPK activation. MAPKs are constitutively activated upon overexpression of AtSOBIR1 (Wu et al., 2018) and the IB shows that MAPK activation by overexpression of AtSOBIR1 is reduced in plants inoculated with pTRV2:NbSERK3a/b, but not in pTRV2:GUSinoculated plants (Fig. 3C, A).

Previously, it has been shown that the accumulation levels of AtSOBIR1 variants appear to be inversely correlated with their constitutive immune activity (Wu et al., 2018). Interestingly, although showing less constitutive immune activity, AtSOBIR1 accumulates to slightly lower levels in leaves of pTRV2:NbSERK3a/b-inoculated $N$. benthamiana plants, when compared to pTRV2:GUS-inoculated plants in which AtSOBIR1 shows strong immune activity. This indicates that, in addition to their role in mediating constitutive immune activity of AtSOBIR1, these BAK1 homologues are also important for proper accumulation of SOBIR1 (Fig. 3E, F).

Together, these experiments show that SOBIR1 forms heterodimers with BAK1, and that SOBIR1 constitutive immunity, as well as its accumulation, depend on BAK1.

\section{SOBIR1-mediated immunity is dependent on kinase-active BAK1}

To further elucidate by which mechanism(s) BAK1 regulates the constitutive immune activity of AtSOBIR1, transient co-transformations of AtSOBIR1 with (untagged) BAK1 variants, mutated in their kinase domain, were performed. These experiments revealed a dominant-negative effect of the BAK1 mutant AtBAK1 ${ }^{\text {C408Y }}$ (also known as AtBAK1-5), and kinase-dead AtBAK1 ${ }^{\mathrm{D} 416 \mathrm{~N}}$ (Schwessinger et al., 2011), in the form of suppression of AtSOBIR1 constitutive immune activity in both $N$. benthamiana and tobacco (Fig. 4A, S2A, S2B). Similarly, the Cf-4/Avr4-induced HR was suppressed 
by co-expression of AtBAK $1^{\mathrm{C} 408 \mathrm{Y}}$ and AtBAK $1^{\mathrm{D} 16 \mathrm{~N}}$ with Avr4 in N. benthamiana:Cf-4 plants (Fig. 4B, S2C). AtBAK $1{ }^{\mathrm{C} 408 Y}$ is known to compromise immunity mediated by FLS2 and EFR, but does not affect brassinosteroid signalling or cell death induction (Schwessinger et al., 2011). AtBAK $1^{\mathrm{D} 416 \mathrm{~N}}$ exhibits a broad loss-of-function phenotype, as this mutant has lost its ability to signal in FLS2- and EFR-mediated immune signalling, as well as in brassinosteroid and cell death signalling (Schwessinger et al., 2011). Our results suggest that the transiently overexpressed AtBAK1 mutants out-compete endogenous functional BAK1 homologues, resulting in compromised AtSOBIR1 constitutive immunity. These findings show that constitutive immunity of AtSOBIR1 depends on defence signalling-competent BAK1.

(A)

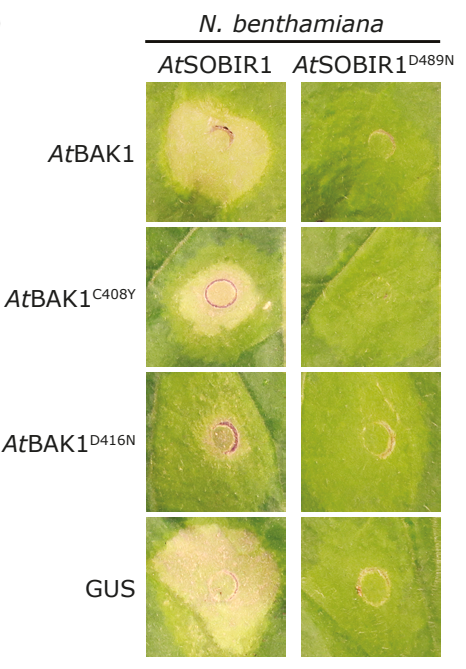

(C)
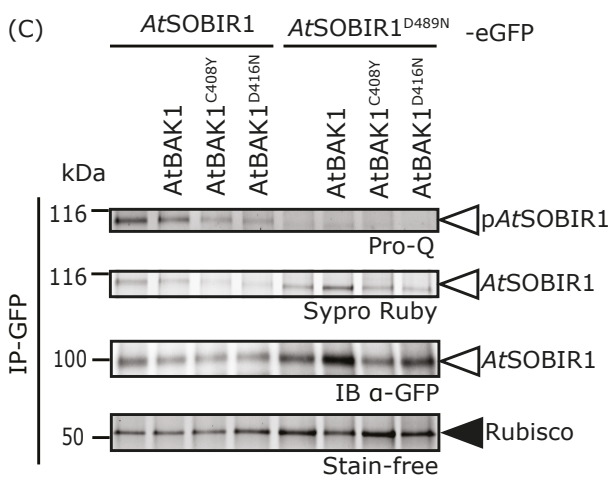

(B)

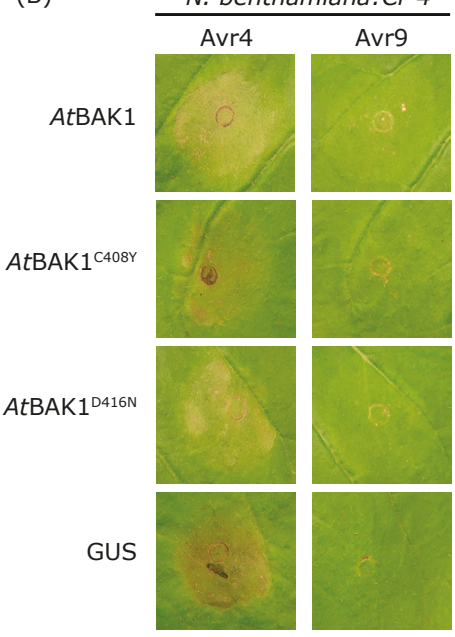

(D)

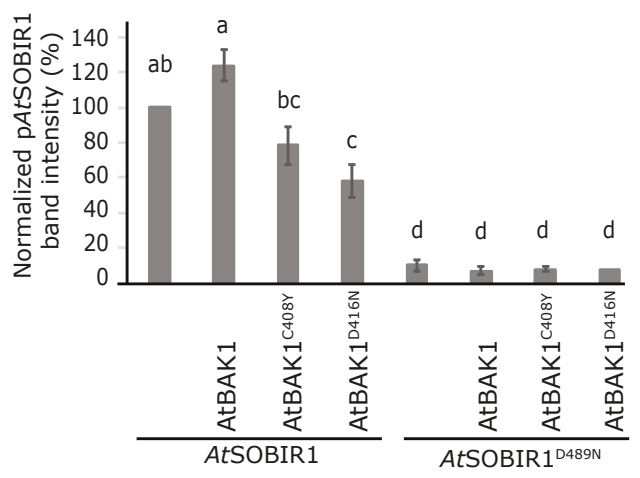

Figure 4. SOBIR1-mediated immunity is dependent on kinase-active BAK1. (A) Transient co-expression of AtSOBIR1 with the AtBAK $1^{\mathrm{C} 408 \mathrm{Y}}$ or AtBAK $1^{\mathrm{D} 416 \mathrm{~N}}$ mutant, results in reduced AtSOBIR1 constitutive immune activity, as compared to co-expression with wild-type AtBAK1 or GUS. The indicated constructs were agroinfiltrated in $N$. benthamiana at an $\mathrm{OD}_{600} 0.5$, with co-infiltration of $\mathrm{P} 19$ at an $\mathrm{OD}_{600}$ of 1 . Pictures were taken at $3 \mathrm{dpi}$, and are representative for two repetitions, with eight agroinfiltrated leaves per sample. See 
also Fig. S2A, B. (B) Transient co-expression of Avr4 in N. benthamiana:Cf-4 with the mutants AtBAK1 $1^{\mathrm{C} 408 \mathrm{~B}}$ or AtBAK1 ${ }^{D 416 \mathrm{~N}}$ results in reduced $\mathrm{Cf}-4 / \mathrm{Avr} 4-\mathrm{HR}$, as compared to co-expression with wild-type AtBAK1 or GUS. The indicated constructs were agroinfiltrated at an $\mathrm{OD}_{600}$ of 0.5 , with co-infiltration of Avr4 or Avr9 at an $\mathrm{OD}_{600}$ of 0.03 . Pictures were taken at $3 \mathrm{dpi}$, and are representative for three repetitions, with agroinfiltration of at least six leaves per sample. See also Fig. S2C. (C) Phosphorylation of AtSOBIR1 is dependent on kinase-active BAK1. The indicated eGFP-tagged AtSOBIR1 and untagged BAK1 constructs were co-agroinfiltrated at an $\mathrm{OD}_{600}$ of 0.5 , in combination with $\mathrm{P} 19$ at an $\mathrm{OD}_{600}$ of 1 . At 2 dpi, leaves were harvested and subjected to IP using GFP-affinity beads, and proteins were subsequently detected by anti GFP IB and Pro-Q stain. The Rubisco background-band of the IP samples is depicted to show equal loading. The experiment was performed twice, and representative results are shown. (D) Quantification of the band intensity of phosphorylated AtSOBIR1 as shown in C. Ratios were obtained by dividing the band intensity of pAtSOBIR1 from the Pro-Q staining, by the band intensity reflecting the total amount of pulled-down AtSOBIR1 in the Sypro Ruby-staining. Data are represented as mean +/- SE. The band intensity of the control sample, expression of AtSOBIR1 without co-expression of BAK1, was set at $100 \%$. The letters indicate significant differences at $\mathrm{p}<0.05$, as determined by one-way ANOVA, including a Tukey post hoc-test.

To determine whether co-expression of the signalling-incompetent BAK1 mutants affects the phosphorylation status of AtSOBIR1, we co-agroinfiltrated AtSOBIR1-eGFP either with wild-type BAK1 or with the different BAK1 mutants in $N$. benthamiana, in the presence of P19. Subsequent immunoprecipitation of AtSOBIR1, followed by Pro-Q staining, revealed a reduction in the phosphorylation level of AtSOBIR1 when co-expressed with AtBAK $1^{\mathrm{C} 408 \mathrm{~V} Y}$ or AtBAK $1^{\mathrm{D} 416 \mathrm{~N}}$, as compared to its co-expression with wild-type BAK1 (Fig. 4C, 4D). This suggests that indeed the high phosphorylation level of AtSOBIR1 is the result of transphosphorylation of AtSOBIR1 by BAK1.

Taken together, these data show that the elevated phosphorylation status of constitutively-active SOBIR1 depends on signalling-competent BAK1. This suggests that immune signalling by SOBIR1 involves trans-phosphorylation events with BAK1.

\section{Discussion}

BAK1 is a well-known co-receptor for ligand-binding RLKs, such as BRI1, EFR, and FLS2 (Chinchilla et al., 2009), and was recently also found to be recruited to activated RLP/ SOBIR1-complexes that are proposed to function as two-component RLKs (Liebrand et al., 2014; Albert et al., 2015; Postma et al., 2016; Wang et al., 2018; Domazakis et al., 2018). Here we show that SOBIR1 and BAK1 act together to signal for defence. We show that, next to kinase-active SOBIR1, kinase-active BAK1 is essential for AtSOBIR1 constitutive immune activity, for strong phosphorylation of AtSOBIR1, and for the Cf-4/Avr4-triggered HR. Based on our findings, we propose a model (Fig. 5) in which AtSOBIR1 trans-autophosphorylates, and transphosphorylates BAK1 thereby activating this co-receptor (Wang et al., 2008; Yan et al., 2012; Somssich et al., 2015). Subsequent transphosphorylation and full activation of AtSOBIR1 by activated BAK 1 enables AtSOBIR1-induced constitutive immunity (Fig. 5A). The model also applies to 
Cf-4/Avr4-mediated immunity, where recognition of Avr4 by Cf-4 possibly initiates SOBIR1 trans-autophosphorylation, subsequent transphosphorylation of BAK1 by SOBIR1, and in its turn transphosphorylation of SOBIR1 by activated BAK1 to fully activate SOBIR1 (Fig. 5B). Fully activated SOBIR1 is then able to initiate further downstream signalling.

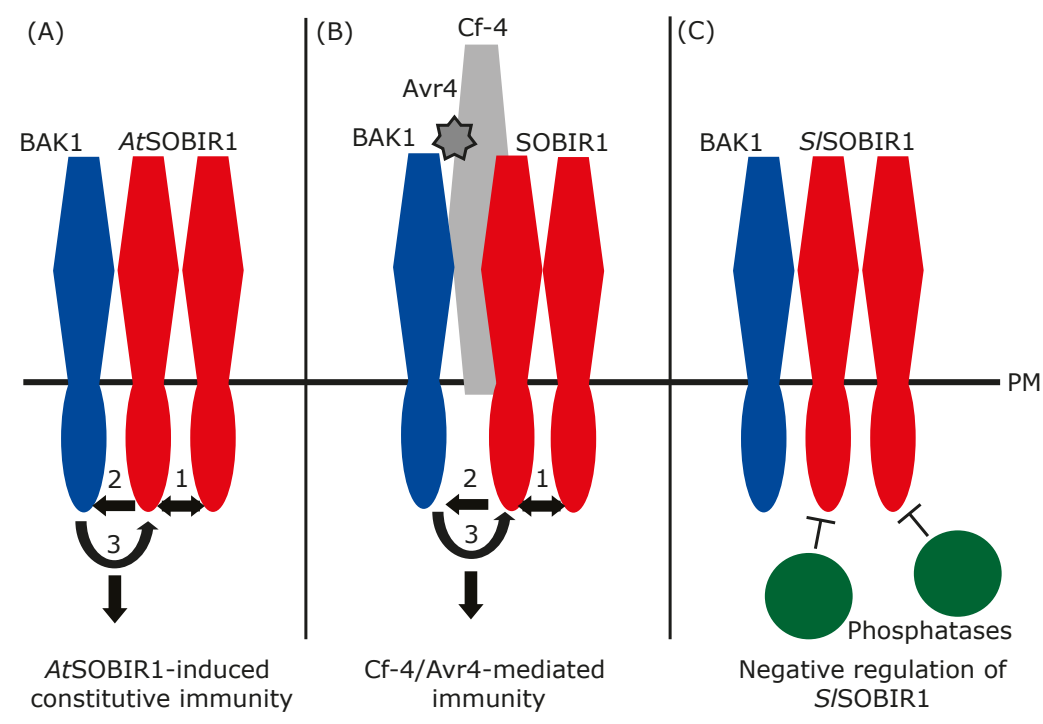

Figure 5. SOBIR1 and BAK1 act together to signal for defence. In this model we propose that SOBIR1 and BAK1 act together to signal for downstream defence activation. Next to trans-autophosphorylation of AtSOBIR1, it is likely that AtSOBIR1 transphosphorylates BAK1. By transphosphorylating BAK1 this coreceptor becomes activated, followed by transphosphorylation and activation of AtSOBIR1 by activated BAK1 to enable AtSOBIR1-induced constitutive immunity (A) and Cf-4/Avr4-mediated HR (B). The arrows represent auto- or transphosphorylation events, and the numbers indicate the proposed order of the various phosphorylation events. Phosphorylation and activity of SISOBIR1 is likely to be downregulated by endogenous phosphatases, when not activated by Avr4 (C). PM, plasma membrane.

First of all, we found that the phosphorylation status of SOBIR1 and its constitutive immune activity positively correlate, as constitutively active AtSOBIR 1 is clearly phosphorylated, and phosphorylation of kinase-dead AtSOBIR1 is not apparent (Fig. 1). Additionally, we observed suppressed phosphorylation of AtSOBIR1 when coexpressed with signalling-incompetent BAK1-mutants (Fig. 4). This indicates that BAK1 plays a role in the transphosphorylation and activation of AtSOBIR1. Knockdown of the expression of BAK 1 homologues NbSERK 1 and NbSERK $3 a / b$ in $N$. benthamiana causes AtSOBIR1 to accumulate to lower levels (Fig. 3E), which might also at least partially explain the compromised AtSOBIR1-induced constitutive immunity in these NbSERK3a/b-silenced plants. Stabilization of RLKs and RLPs by regulatory RLKs has been observed before, so it is likely that SOBIR1 and BAK1 promote each other's 
accumulation (Wang et al., 2008; Liebrand et al., 2013; Imkampe et al., 2017). Because silencing of $B A K 1$ homologues suppressed the accumulation of SOBIR1, attempts to measure changes in the phosphorylation level of SOBIR1 upon silencing of $B A K 1$ homologues were not successful.

A constitutive interaction between SOBIR1 and BAK1 has already earlier been suggested to occur (Liu et al., 2016; Postma et al., 2016). Although Liu and coworkers could not show an interaction between AtSOBIR1 and AtBAK1 in $N$. benthamiana, they could do so in Arabidopsis upon silencing of a negative regulator of defence, AtBIR1 (Gao et al., 2009; Liu et al., 2016). Silencing BIR1 led to more BAK1 availability, and a more clear interaction of BAK1 with SOBIR1 (Liu et al., 2016). In the experiments that we describe here, the accumulation of AtSOBIR1 is relatively low due to its constitutive immune activity in N. benthamiana (Wu et al., 2018), and an interaction between AtBAK1 and AtSOBIR1 is not visible (Fig. 3A). For AtSOBIR1D489N and SISOBIR1 we did observe an interaction with BAK1, as these SOBIR1 variants accumulate to higher amounts because they do not trigger a constitutive cell death response. A constitutive interaction between SOBIR1 and BAK1 is anticipated to occur only at very low levels, as this interaction is specifically stimulated upon ligand perception by the SOBIR1-associated RLP, in order to activate the immune system (Postma et al., 2016; Albert et al., 2015; Wang et al., 2018; Domazakis et al., 2018). Pre-formation of immune complexes at nanoclusters on the plasma membrane, without their activation by the matching ligand, has been shown before (Jarsch et al., 2014; Somssich et al., 2015; Bücherl et al., 2017). It was found that various preformed immune complexes are present at the plasma membrane, spatially separated into different nanoclusters. Such a separation into different nanodomains enables quick and diverse responses. Thus, a small pool of pre-formed RLP/SOBIR1/BAK1 complexes, probably kept in check by endogenous phosphatases (Couto \& Zipfel, 2016; Wu et al., 2018), might enable quick and specific responses to elicitation in resistant plants.

Next to sequestering of co-receptors required for downstream signalling, immune receptor activity is also regulated at the level of phosphorylation (Couto \& Zipfel, 2016). For instance, BAK1 and FLS2 are negatively regulated by type 2A and type $2 \mathrm{C}$ Protein Phosphatases (PP2A and PP2C), respectively (Segonzac et al., 2014; Gómez-Gómez et al., 2001; Couto et al., 2016). We here show that AtSOBIR1, which can constitutively activate immune responses, is highly phosphorylated when overexpressed in $N$. benthamiana, whereas SISOBIR1 is not (Fig. 1A). It is likely that endogenous phosphatases of $N$. benthamiana keep SISOBIR1 in check by dephosphorylation (Fig. $5 C)$, but do not have the appropriate affinity for the phosphorylated kinase domain of AtSOBIR1, and therefore cannot properly dephosphorylate this non-Solanaceous protein, leading to excessive phosphorylation and thereby constitutive immune activation (Wu et al., 2018). 
Similar to BAK1, SOBIR1 is a typical RD-kinase as it contains an arginine (R) and aspartic acid (D) residue in its catalytic site. RD-kinases are generally thought to require phosphorylation of their activation loop to acquire the active conformation (Johnson et al., 1996; Nolen et al., 2004; Kornev et al., 2006). Our observation that kinase-dead AtSOBIR1 ${ }^{\text {D489N }}$ does not show substantial phosphorylation, as compared to wild-type AtSOBIR1, when transiently expressed in N. benthamiana, indicates that AtSOBIR1 needs to trans-autophosphorylate to a certain level in order to become signalling-competent. As SOBIR1 forms homodimers, it is indeed possible that such trans-autophosphorylation takes place (Fig. 2). This trans-autophosphorylation is likely required to keep SOBIR1 in a signalling-active state. Additionally, the lack of apparent phosphorylation of AtSOBIR1 $1^{\text {D489N }}$ possibly means that AtSOBIR1 needs to transphosphorylate its signalling partner BAK1 in order for this partner to become signalling-competent, which then on its turn transphosphorylates AtSOBIR1. Indeed, upon overexpression of BAK1 together with SOBIR1, we observed elevated phosphorylation levels of SOBIR1 (Fig. 4C, 4D). Moreover, overexpression of AtSOBIR1 with signalling-incompetent BAK1 mutants, which are unable to signal for defence, resulted in reduced phosphorylation levels of SOBIR1. These observations indicate that for full phosphorylation of SOBIR1, and immune signalling by SOBIR1, transphosphorylation by BAK1 is necessary. This is in concert with previous studies on ligand binding RLKs and BAK1, for which it was concluded that transphosphorylation of the RLK and BAK1 is necessary to initiate signalling upon ligand perception (Wang et al., 2008; Yan et al., 2012). It is likely that, upon ligand recognition by an RLP, the RLP and associated SOBIR1 undergo conformational changes, leading to the ability of SOBIR1 to trans-phosphorylate recruited BAK1. This trans-phosphorylation event locks BAK1 in the active conformation (Yan et al., 2012) and provides BAK1 with the capacity to transphosphorylate and fully activate SOBIR1. The resulting activated complex subsequently is able to trigger downstream defence signalling (Fig. 5).

Interestingly, we observed reduced AtSOBIR1 phosphorylation levels and cell death upon overexpression of AtBAK $1^{\text {C408Y }}$ (also referred to as BAK1-5) together with AtSOBIR1 (Fig. 4). AtBAK $1{ }^{\text {C408Y }}$ was initially found to be impaired in complementing for defence signalling by FLS2 and EFR (Schwessinger et al., 2011). Nevertheless, AtBAK $1^{\text {C408Y }}$ has only slightly reduced kinase activity, and is not impaired in brassinosteroid or cell death signalling. Therefore, this mutant has been important to unlink the ability of BAK 1 to signal for defence, cell death, and development. We observed that AtBAK $1^{\mathrm{C} 408 \mathrm{Y} Y}$ is impaired in signalling for AtSOBIR1-induced constitutive immunity, as well as in mediating transphosphorylation of AtSOBIR1 (Fig. 4). Thus, AtSOBIR1 specifically signals for immunity together with BAK1. This further confirms that constitutive immune activity induced by AtSOBIR1 is following the same immune pathway as Cf-4/Avr4-mediated HR. 
In conclusion, we here show that AtSOBIR1 and BAK1 act together to signal for defence. Our in planta data support a model in which SOBIR 1 trans-autophosphorylates, and upon elicitation transphosphorylates BAK1, and is transphosphorylated by activated BAK1 to signal for immunity. We envisage that this model not only describes signalling for AtSOBIR1-constitutive immunity, but also signalling upon BAK1 heterodimer formation with bi-partite RLP/SOBIR1 complexes (Fig. 5).

\section{Materials \& Methods}

\section{Binary vectors for Agrobacterium-mediated transformation and virus-induced gene silencing (VIGS)}

The constructs pBIN-KS-35S::AtSOBIR1-eGFP, pBIN-KS-35S::AtSOBIR1 $1^{\text {D489N-eGFP, }}$

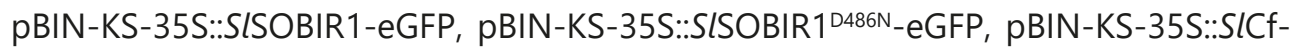
4-eGFP, pGWB20-35S::SISERK3-myc, pGWB20-35s::SISOBIR1-Myc, and pGWB2035s::AtSOBIR1-Myc have been described previously (Liebrand et al., 2012 and 2013). AtBAK1-Myc has been described by Halter et al., (2014). Avr4 was expressed using the pMOG800 construct (Van der Hoorn et al., 2000). P19 (Voinnet et al., 2015), pBIN61GUS, pTRV1 (Liu et al., 2002a and 2002b), pTRV2:GUS (Tameling \& Baulcombe, 2007), and pTRV2:NbSERK3a/b (Heese et al., 2007) have been described elsewhere. AtBAK1, AtBAK $1^{\mathrm{C} 408 \mathrm{r}}$, and AtBAK $1^{\mathrm{D} 416 \mathrm{~N}}$ originate from Schwessinger et al. (2011). GFP-LTI6b has been described previously (Kurup et al., 2005). AtFLS2-eGFP and S/FLS2-eGFP were described by Robatzek et al. (2006 and 2007). SISOBIR1-cYFP, SISOBIR1-nYFP, and SICf-4-cYFP (Postma et al., 2016), and AtFLS2-cYFP and AtFLS2-nYFP (Frei dit Frey et al., 2012) have been described elsewhere.

\section{Plant growth conditions}

Nicotiana tabacum (tobacco) (cv. SR1 and cv. Samsun) and N. benthamiana (wildtype and $N$. benthamiana stably expressing SICf-4 under its native promoter (referred to as $N$. benthamiana:Cf-4, Gabriëls et al., 2006)), were grown under $16 \mathrm{~h}$ light at $25^{\circ} \mathrm{C}$ and $8 \mathrm{~h}$ darkness at $21^{\circ} \mathrm{C}$, at $\sim 75 \%$ relative humidity.

\section{Virus-induced gene silencing (VIGS) in tobacco and $\mathbf{N}$. benthamiana}

VIGS using TRV-based vectors was performed in tobacco (cV. Samsun) and $N$. benthamiana:Cf-4 as described previously (Liebrand et al., 2012; Zhang et al., 2013b).

\section{Agrobacterium-mediated transient transformation}

Agrobacterium-mediated transient transformations (agroinfiltrations) were performed as previously described (Van der Hoorn et al., 2000). Binary constructs expressing affinity-tagged-proteins were agroinfiltrated with Agrobacterium tumefaciens cultures 
at an $\mathrm{OD}_{600}$ of 1 in combination with $\mathrm{P} 19$ at an $\mathrm{OD}_{600}$ of 1 , unless indicated otherwise. Leaves were harvested for protein isolation and immunoprecipitation (IP) at $2 \mathrm{dpi}$, unless indicated otherwise. Percentages of HR were quantified by visual scoring for full HR (100\%), mildly reduced HR (60\%), strongly reduced HR (30\%) and no HR (0\%).

\section{Immunoprecipitations, immunoblotting, phosphorylation analysis, and MAPK activation analysis}

Immunoprecipitations (IPs) and co-IPs were performed as described previously (Liebrand et al., 2013). To detect phosphorylated proteins, a protein extraction buffer was used as described by Karlova and co-workers (2006 and 2009), with minor modifications; instead of Tris and Triton- $X, 100 \mathrm{mM} \mathrm{NaPi}(\mathrm{pH} 7.2)$ and $1 \%$ IGEPAL CA-630 (NP40) were used, respectively. Pre-cast TGX gels were used for Pro-Q and SYPRO Ruby analyses (Bio-Rad \#456-1095). Pro-Q diamond phosphoprotein gel stains and subsequent SYPRO Ruby stains were done according to the manufacturer's recommendations (Invitrogen; Taylor et al., 2013). TGX stain-free gels were used for all other protein analyses (Bio-Rad \#456-8085), and total protein was visualized using the stain-free method or with Coomassie Brilliant Blue (CBB). The following antibodies were used for protein detection on immunoblots (IB): aGFP-HRP (130-091-833, MACS), aMyc (cMyc9E10, sc-40, Santa Cruz), $\alpha$ Mouse-HRP (GE healthcare), anti-p42/p44-erk (NEB) and goat anti-rabbit (Sigma). Band intensities were measured using Image Lab software (Bio-Rad), and ratios were calculated as indicated with the figure. To quantify immunoprecipitated protein bands, the Rubisco-background band in the IP sample was taken as an internal standard for the total protein concentration of the sample.

\section{Protein localization studies}

Confocal laser scanning microscopy (CLSM) was performed using a Leica SP5 laser point scanning microscope (Leica, Germany), mounted with hybrid detectors (HyD) as described previously (Beck et al., 2012). For CLSM analysis of eGFP, constructs were transiently expressed in adult tobacco plants by infiltration with Agrobacterium tumefaciens suspensions of $\mathrm{OD}_{600} 0.3$. At $2 \mathrm{dpi}$, fluorophores were excited using a $488 \mathrm{~nm}$ argon laser and fluorescence emission was captured between 495 and 540 $\mathrm{nm}$. Chloroplast autofluorescence was captured between 700 and $800 \mathrm{~nm}$. For CLSM analysis of bimolecular fluorescence complementation experiments (BiFC, splitYFP), CYFP and nYFP constructs were transiently co-expressed by co-infiltration of adult $N$. benthamiana plants using Agrobacterium tumefaciens suspensions each at $\mathrm{OD} 600=0.3$. Reconstituted YFP molecules were excited using a $514 \mathrm{~nm}$ argon laser, and fluorescence emission was captured between 520-550 nm. Chloroplast autofluorescence was captured between 700 and $800 \mathrm{~nm}$. Images were taken using the 20x objective (for eGFP) or 40x objective (for YFP) and were processed using Leica LAS-AF and FIJI (ImageJ) software packages. 


\section{Acknowledgements}

We thank Unifarm personnel for excellent plant care. We acknowledge Maria Font Farré for initiating experiments on the dominant-negative effect of BAK1, and Cris Wijnen for help with statistics. A.M.v.d.B. is supported by the Netherlands Organization for Scientific Research (NWO), Earth and Life Sciences (ALW). Research by J.P. and S.R. is supported by the Gatsby Charitable Foundation. 


\section{References}

Albert, I., Böhm, H., Albert, M., Feiler, C.E., Imkampe, J., Wallmeroth, N., Brancato, C., Raaymakers, T.M., Oome, S., Zhang, H., Krol, E., Grefen, C., Gust, A.A., Chai, J., Hedrich, R., Van den Ackerveken, G. and Nürnberger, T. (2015) An RLP23-SOBIR1-BAK1 complex mediates NLPtriggered immunity. Nature Plants 1, 15140.

Bar, M., Sharfman, M., Ron, M. and Avni, A. (2010) BAK1 is required for the attenuation of ethyleneinducing xylanase (Eix)-induced defense responses by the decoy receptor LeEix1. The Plant Journal 63, 791-800.

Beck, M., Zhou, J., Faulkner, C., MacLean, D. and Robatzek, S. (2012) Spatio-temporal cellular dynamics of the Arabidopsis flagellin receptor reveal activation status-dependent endosomal sorting. The Plant Cell 24, 4205-4219.

Bi, G., Liebrand, T.W., Cordewener, J.H., America, A.H., Xu, X. and Joosten, M.H.A.J. (2014) Arabidopsis thaliana receptor-like protein AtRLP23 associates with the receptor-like kinase AtSOBIR1. Plant Signaling \& Behavior 9, e27937.

Bi, G., Liebrand, T.W.H., Bye, R.R., Postma, J., van der Burgh, A.M., Robatzek, S., Xu, X. and Joosten, M.H.A.J. (2015) SOBIR1 requires the GxxxG dimerization motif in its transmembrane domain to form constitutive complexes with receptor-like proteins. Molecular Plant Pathology 17, 96-107.

Böhm, H., Albert, I., Fan, L., Reinhard, A. and Nürnberger, T. (2014) Immune receptor complexes at the plant cell surface. Current Opinion in Plant Biology 20, 47-54.

Bücherl, C.A., Jarsch, I.K., Schudoma, C., Segonzac, C., Mbengue, M., Robatzek, S., MacLean, D., Ott, T. and Zipfel, C. (2017) Plant immune and growth receptors share common signalling components but localise to distinct plasma membrane nanodomains. eLife 6, e25114

Catanzariti, A.M., Do, H.T., Bru, P., Sain, M., Thatcher, L.F., Rep, M. and Jones, D.A. (2017) The tomato I gene for Fusarium wilt resistance encodes an atypical leucine-rich repeat receptor-like protein whose function is nevertheless dependent on SOBIR1 and SERK3/BAK1. The Plant Journal 89, 11951209.

Chinchilla, D., Shan, L., He, P., de Vries, S. and Kemmerling, B. (2009) One for all: the receptorassociated kinase BAK1. Trends in Plant Science 14, 535-541.

Chinchilla, D., Zipfel, C., Robatzek, S., Kemmerling, B., Nürnberger, T., Jones, J.D., Felix, G. and Boller, T. (2007) A flagellin-induced complex of the receptor FLS2 and BAK1 initiates plant defence. Nature 448, 497-500.

Couto, D. and Zipfel, C. (2016) Regulation of pattern recognition receptor signalling in plants. Nature Reviews Immunology 16, 537-552.

Domazakis, E., Wouters, D., Visser, R., Kamoun, S., Joosten, M.H.A.J. and Vleeshouwers, V.G.A.A. (2018) The ELR-SOBIR1 complex functions as a two-component RLK to mount defense against Phytophthora infestans. Molecular Plant-Microbe Interactions. Doi 10.1094/mpmi-09-17-0217-r

Dufayard, J.-F., Bettembourg, M., Fischer, I., Droc, G., Guiderdoni, E., Périn, C., Chantret, N. and Diévart, A. (2017) New insights on leucine-rich repeats receptor-like kinase orthologous relationships in angiosperms. Frontiers in Plant Science 8. 381

Fischer, I., Diévart, A., Droc, G., Dufayard, J.-F. and Chantret, N. (2016) Evolutionary dynamics of the Leucine-Rich Repeats Receptor-Like Kinase (LRR-RLK) subfamily in angiosperms. Plant physiology. doi. org/10.1104/pp.15.01470

Fradin, E.F., Zhang, Z., Ayala, J.C.J., Castroverde, C.D.M., Nazar, R.N., Robb, J., Liu, C.M. and Thomma, B.P.H.J. (2009) Genetic dissection of Verticillium wilt resistance mediated by tomato Ve1. Plant Physiology 150, 320-332.

Frei dit Frey, N., Mbengue, M., Kwaaitaal, M., Nitsch, L., Altenbach, D., Haweker, H., Lonzano-Duran, R., Njo, M.F., Beeckman, T. and Huettel, B. (2012) Plasma membrane calcium ATPases are important components of receptor-mediated signalling in plant immune responses and development. Plant Physiology 159, 798 - 809. 
Frescatada-Rosa, M., Robatzek, S. and Kuhn, H. (2015) Should I stay or should I go? Traffic control for plant pattern recognition receptors. Current Opinion in Plant Biology 28, 23-29.

Gabriëls, S.H.E.J., Takken, F.L.W., Vossen, J.H., De Jong, C.F., Liu, Q., Turk, S.C.H.J., Wachowski, L.K., Peters, J., Witsenboer, H.M.A., De Wit, P.J.G.M. and Joosten, M.H.A.J. (2006) cDNA-AFLP combined with functional analysis reveals novel genes involved in the hypersensitive response. Molecular PlantMicrobe Interactions 19, 567-576.

Gao, M., Wang, X., Wang, D., Xu, F., Ding, X., Zhang, Z., Bi, D., Cheng, Y.T., Chen, S., Li, X. and Zhang, Y. (2009) Regulation of cell death and innate immunity by two receptor-like kinases in Arabidopsis. Cell Host and Microbe 6, 34-44.

Gómez-Gómez, L., Bauer, Z. and Boller, T. (2001) Both the extracellular leucine-rich repeat domain and the kinase activity of FLS2 are required for flagellin binding and signaling in Arabidopsis. The Plant Cell 13, 1155-1163.

Gómez-Gómez, L. and Boller, T. (2000) FLS2: an LRR receptor-like kinase involved in the perception of the bacterial elicitor flagellin in Arabidopsis. Molecular Cell 5, 1003-1011.

Gust, A.A. and Felix, G. (2014) Receptor like proteins associate with SOBIR1-type of adaptors to form bimolecular receptor kinases. Current Opinion in Plant Biology 21, 104-111.

Heese, A., Hann, D.R., Gimenez-Ibanez, S., Jones, A.M.E., He, K., Li, J., Schroeder, J.I., Peck, S.C. and Rathjen, J.P. (2007) The receptor-like kinase SERK3/BAK1 is a central regulator of innate immunity in plants. Proceedings of the National Academy of Sciences of the United States of America 104, 1221712222.

Hegenauer, V., Fürst, U., Kaiser, B., Smoker, M., Zipfel, C., Felix, G., Stahl, M. and Albert, M. (2016) Detection of the plant parasite Cuscuta reflexa by a tomato cell surface receptor. Science 353, 478-481.

Imkampe, J., Halter, T., Huang, S., Schulze, S., Mazzotta, S., Schmidt, N., Manstretta, R., Postel, S., Wierzba, M., Yang, Y., vanDongen, W.M., Stahl, M., Zipfel, C., Goshe, M.B., Clouse, S., de Vries, S.C., Tax, F., Wang, X. and Kemmerling, B. (2017) The Arabidopsis leucine-rich repeat receptor kinase BIR3 negatively regulates BAK1 receptor complex formation and stabilizes BAK1. The Plant Cell 29.

Jarsch, I.K., Konrad, S.S.A., Stratil, T.F., Urbanus, S.L., Szymanski, W., Braun, P., Braun, K.-H. and Ott, T. (2014) Plasma membranes are subcompartmentalized into a plethora of coexisting and diverse microdomains in Arabidopsis and Nicotiana benthamiana. The Plant Cell Online 26.

Jehle, A.K., Fürst, U., Lipschis, M., Albert, M. and Felix, G. (2013) Perception of the novel MAMP eMax from different Xanthomonas species requires the Arabidopsis receptor-like protein ReMAX and the receptor kinase SOBIR. Plant Signaling \& Behavior 8, e27408.

Johnson, L.N., Noble, M.E.M. and Owen, D.J. (1996) Active and inactive protein kinases: Structural basis for regulation. Cell 85, 149-158.

Karlova, R., Boeren, S., Russinova, E., Aker, J., Vervoort, J. and de Vries, S. (2006) The Arabidopsis somatic embryogenesis receptor-like kinase1 protein complex includes brassinosteroid-insensitive1. The Plant Cell 18, 626-638.

Karlova, R., Boeren, S., van Dongen, W., Kwaaitaal, M., Aker, J., Vervoort, J. and de Vries, S. (2009) Identification of in vitro phosphorylation sites in the Arabidopsis thaliana somatic embryogenesis receptor-like kinases. Proteomics 9, 368-379.

Kornev, A.P., Haste, N.M., Taylor, S.S. and Ten Eyck, L.F. (2006) Surface comparison of active and inactive protein kinases identifies a conserved activation mechanism. Proceedings of the National Academy of Sciences of the United States of America 103, 17783-17788.

Kurup, S., Runions, J., Köhler, U., Laplaze, L., Hodge, S. and Haseloff, J. (2005) Marking cell lineages in living tissues. The Plant Journal 42, 444-453.

Leslie, M.E., Lewis, M.W., Youn, J.Y., Daniels, M.J. and Liljegren, S.J. (2010) The EVERSHED receptor-like kinase modulates floral organ shedding in Arabidopsis. Development 137, 467-476.

Li, J. and Chory, J. (1997) A putative leucine-rich repeat receptor kinase involved in brassinosteroid signal transduction. Cell 90, 929-938. 
Liebrand, T.W.H., Smit, P., Abd-El-Haliem, A., de Jonge, R., Cordewener, J.H.G., America, A.H.P., Sklenar, J., Jones, A.M.E., Robatzek, S., Thomma, B.P.H.J., Tameling, W.I.L. and Joosten, M.H.A.J. (2012) Endoplasmic reticulum-quality control chaperones facilitate the biogenesis of Cf receptor-like proteins involved in pathogen resistance of tomato. Plant Physiology 159, 1819-1833.

Liebrand, T.W.H., van den Berg, G.C.M., Zhang, Z., Smit, P., Cordewener, J.H.G., America, A.H.P., Sklenar, J., Jones, A.M.E., Tameling, W.I.L., Robatzek, S., Thomma, B.P.H.J. and Joosten, M.H.A.J. (2013) Receptor-like kinase SOBIR1/EVR interacts with receptor-like proteins in plant immunity against fungal infection. Proceedings of the National Academy of Sciences of the United States of America 110, 10010-10015.

Liebrand, T.W.H., van den Burg, H.A. and Joosten, M.H.A.J. (2014) Two for all: receptor-associated kinases SOBIR1 and BAK1. Trends in Plant Science 19, 123-132.

Liu, Y., Huang, X., Li, M., He, P. and Zhang, Y.C. (2016) Loss-of-function of Arabidopsis receptorlike kinase BIR1 activates cell death and defense responses mediated by BAK1 and SOBIR1. New Phytologist 212, 637-645.

Liu, Y., Schiff, M. and Dinesh-Kumar, S. (2002a) Virus-induced gene silencing in tomato. The Plant Journal 31, 777-786.

Liu, Y., Schiff, M., Marathe, R. and Dinesh-Kumar, S. (2002b) Tobacco Rar1, EDS1 and NPR1/NIM1 like genes are required for $\mathrm{N}$-mediated resistance to tobacco mosaic virus. Plant Journal 30, 415-429.

Ma, L. and Borhan, M.H. (2015) The receptor-like kinase SOBIR1 interacts with Brassica napus LepR3 and is required for Leptosphaeria maculans AvrLm1-triggered immunity. Frontiers in Plant Science 6, 933.

Macho, A.P., Lozano-Durán, R. and Zipfel, C. (2015) Importance of tyrosine phosphorylation in receptor kinase complexes. Trends in Plant Science 20, 269-272.

Macho, A.P., Schwessinger, B., Ntoukakis, V., Brutus, A., Segonzac, C., Roy, S., Kadota, Y., Oh, M.H., Sklenar, J., Derbyshire, P., Lozano-Durán, R., Gro Malinovsky, F., Monaghan, J., Menke, F.L., Huber, S.C., Yang He, S. and Zipfel, C. (2014) A bacterial tyrosine phosphatase inhibits plant pattern recognition receptor activation. Science 343, 1509-1512.

Macho, A.P. and Zipfel, C. (2014) Plant PRRs and the activation of innate immune signaling. Molecular Cell 54, 263-272.

Nam, K.H. and Li, J. (2002) BRI1/BAK1, a receptor kinase pair mediating brassinosteroid signaling. Cell 110, 203-212.

Nolen, B., Taylor, S. and Ghosh, G. (2004) Regulation of protein kinases: Controlling activity through activation segment conformation. Molecular Cell 15, 661-675.

Oh, M.H., Wu, X., Clouse, S.D. and Huber, S.C. (2011) Functional importance of BAK1 tyrosine phosphorylation in vivo. Plant Signaling \& Behavior 6, 400-405.

Postma, J., Liebrand, T.W.H., Bi, G., Evrard, A., Bye, R.R., Mbengue, M., Kuhn, H., Joosten, M.H.A.J. and Robatzek, S.C. (2016) Avr4 promotes Cf-4 receptor-like protein association with the BAK1/ SERK3 receptor-like kinase to initiate receptor endocytosis and plant immunity. New Phytologist 210, 627-642.

Robatzek, S., Bittel, P., Chinchilla, D., Köchner, P., Felix, G., Shiu, S.-H. and Boller, T. (2007) Molecular identification and characterization of the tomato flagellin receptor LeFLS2, an orthologue of Arabidopsis FLS2 exhibiting characteristically different perception specificities. Plant Molecular Biology 64, 539-547.

Robatzek, S., Chinchilla, D. and Boller, T. (2006) Ligand-induced endocytosis of the pattern recognition receptor FLS2 in Arabidopsis. Genes \& Development 20, 537-542.

Santiago, J., Henzler, C. and Hothorn, M. (2013) Molecular mechanism for plant steroid receptor activation by somatic embryogenesis co-receptor kinases. Science 341, 889-892.

Schwessinger, B., Roux, M., Kadota, Y., Ntoukakis, V., Sklenar, J., Jones, A. and Zipfel, C. (2011) Phosphorylation-dependent differential regulation of plant growth, cell death, and innate immunity by the regulatory receptor-like kinase BAK1. PLoS Genetics 7, e1002046-e1002046. 
Segonzac, C., Macho, A.P., Sanmartín, M., Ntoukakis, V., Sánchez-Serrano, J.J. and Zipfel, C. (2014) Negative control of BAK1 by protein phosphatase 2A during plant innate immunity. The EMBO Journal 33, 2069-2079.

Somssich, M., Ma, Q., Weidtkamp-Peters, S., Stahl, Y., Felekyan, S., Bleckmann, A., Seidel, C.A. and Simon, R. (2015) Real-time dynamics of peptide ligand-dependent receptor complex formation in planta. Science Signaling 8, DOI: 10.1126/scisignal.aab0598.

Sun, W., Cao, Y., Labby, K.J., Bittel, P., Boller, T. and Bent, A.F. (2012) Probing the Arabidopsis flagellin receptor: FLS2-FLS2 association and the contributions of specific domains to signaling function. The Plant Cell 24, 1096-1113.

Sun, Y., Han, Z., Tang, J., Hu, Z., Chai, C., Zhou, B. and Chai, J. (2013a) Structure reveals that BAK1 as a co-receptor recognizes the BRI1-bound brassinolide. Cell Research 23, 1326.

Sun, Y., Li, L., Macho, A.P., Han, Z., Hu, Z., Zipfel, C., Zhou, J.M. and Chai, J. (2013b) Structural basis for flg22-induced activation of the Arabidopsis FLS2-BAK1 immune complex. Science 342, 624-628.

Tameling, W.I.L. and Baulcombe, D.C. (2007) Physical association of the NB-LRR resistance protein Rx with a Ran GTPase-activating protein is required for extreme resistance to Potato virus X. The Plant Cell 19, 1682-1694.

Taylor, I., Seitz, K., Bennewitz, S. and Walker, J.C. (2013) A simple in vitro method to measure autophosphorylation of protein kinases. Plant Methods $\mathbf{9}, 22$.

van der Hoorn, R.A.L., Laurent, F., Roth, R. and de Wit, P.J.G.M. (2000) Agroinfiltration is a versatile tool that facilitates comparative analyses of Avr9/Cf-9-induced and Avr4/Cf-4-induced necrosis. Molecular Plant-Microbe Interactions 13, 439-446.

Voinnet, O., Rivas, S., Mestre, P. and Baulcombe, D.C. (2015) Retraction: An enhanced transient expression system in plants based on suppression of gene silencing by the p19 protein of tomato bushy stunt virus (retraction of The Plant Journal 33, pg 949, 2003).

Wang, X., Kota, U., He, K., Blackburn, K., Li, J., Goshe, M.B., Huber, S.C. and Clouse, S.D. (2008) Sequential transphosphorylation of the BRI1/BAK1 receptor kinase complex impacts early events in brassinosteroid signaling. Developmental Cell 15, 220-235.

Wang, Y., Xu, Y., Sun, Y., Wang, H., Qi, J., Wan, B., Ye, W., Lin, Y., Shao, Y., Dong, S., Tyler, B.M. and Wang, Y. (2018) Leucine-rich repeat receptor-like gene screen reveals that Nicotiana RXEG1 regulates glycoside hydrolase 12 MAMP detection. Nature Communications $\mathbf{9}, 594$.

Wu, J., van der Burgh, A., Bi, G., Zhang, L., Alfano, J.R., Martin, G. and Joosten, M.H.A.J. (2018) The bacterial effector AvrPto targets the regulatory co-receptor SOBIR1 and suppresses defence signalling mediated by the receptor-like protein Cf-4. Molecular Plant-Microbe Interactions 31, 7585.

Yan, L., Ma, Y., Liu, D., Wei, X., Sun, Y., Chen, X., Zhao, H., Zhou, J., Wang, Z., Shui, W. and Lou, Z. (2012) Structural basis for the impact of phosphorylation on the activation of plant receptor-like kinase BAK1. Cell Research 22, 1304-1308.

Zhang, L., Kars, I., Essenstam, B., Liebrand, T.W., Wagemakers, L., Elberse, J., Tagkalaki, P., Tjoitang, D., van den Ackerveken, G. and van Kan, J.A. (2014) Fungal endopolygalacturonases are recognized as microbe-associated molecular patterns by the Arabidopsis receptor-like protein RESPONSIVENESS TO BOTRYTIS POLYGALACTURONASES1. Plant Physiology 164, 352-364.

Zhang, W., Fraiture, M., Kolb, D., Löffelhardt, B., Desaki, Y., Boutrot, F.F.G., Tör, M., Zipfel, C., Gust, A.A. and Brunner, F. (2013a) Arabidopsis receptor-like protein30 and receptor-like kinase Suppressor Of BIR1-1/EVERSHED mediate innate immunity to necrotrophic fungi. The Plant Cell 25, 4227-4241.

Zhang, Z., Fradin, E., de Jonge, R., van Esse, H.P., Smit, P., Liu, C.-M. and Thomma, B.P.H.J. (2013b) Optimized agroinfiltration and virus-induced gene silencing to study Ve1-mediated Verticillium resistance in tobacco. Molecular Plant-Microbe Interactions 26, 182-190.

Zipfel, C. (2014) Plant pattern-recognition receptors. Trends in Immunology 35, 345-351.

Zipfel, C., Kunze, G., Chinchilla, D., Caniard, A., Jones, J.D., Boller, T. and Felix, G. (2006) Perception of the bacterial PAMP EF-Tu by the receptor EFR restricts Agrobacterium-mediated transformation. Cell 125, 749-760. 


\section{Supplemental data}

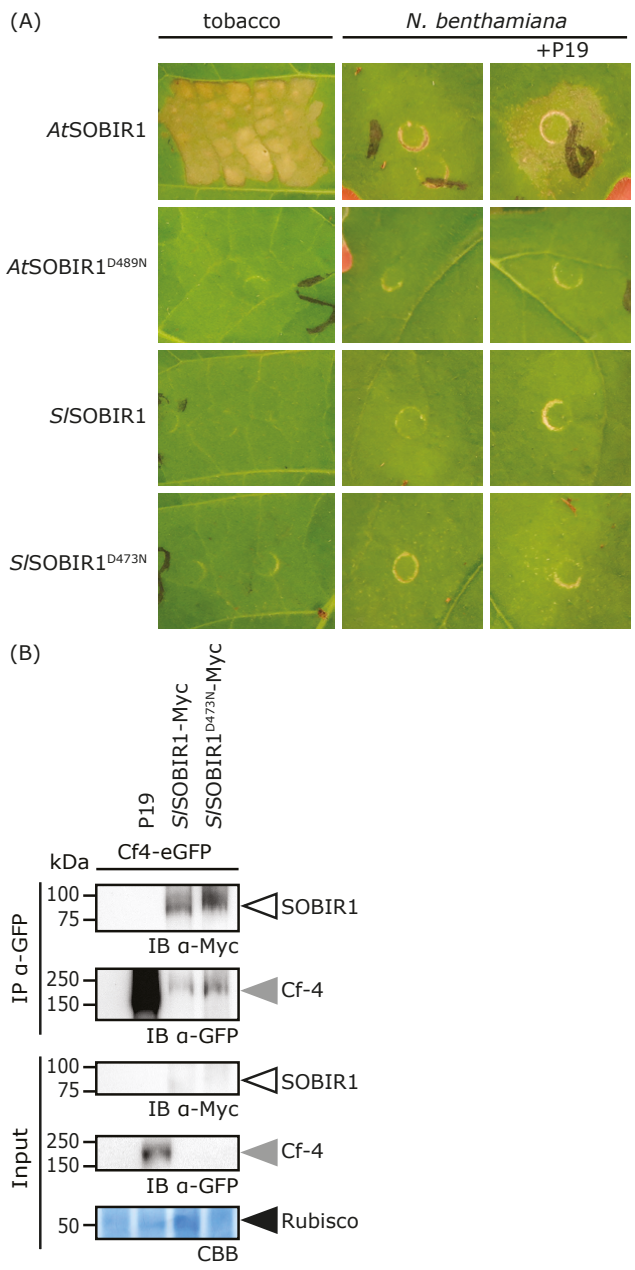

Figure S1. Kinase activity of SOBIR1 is not required for Cf-4 stabilization. (A) Transient overexpression of AtSOBIR1 induces cell death in tobacco, and in N. benthamiana when co-expressed with P19. Agroinfiltrations were done at an $\mathrm{OD}_{600}$ of 1 . Where indicated, $\mathrm{P} 19$ was co-infiltrated also at an $\mathrm{OD}_{600}$ of 1. Pictures were taken at 3 days post infiltration (dpi). Note that constitutive immune activity of AtSOBIR1 requires its kinase activity. Furthermore, overexpression of SISOBIR1 from the Solanaceous plant tomato does not result in cell death. See also Wu et al. (2018). (B) Co-expression of wild-type SISOBIR1 as well as kinase-dead SISOBIR1 ${ }^{\text {D473N }}$ stabilizes $\mathrm{Cf}-4$, when co-expressed in $\mathrm{N}$. benthamiana. Note that the signal of $\mathrm{Cf}-4$ is increased when overexpressed with both wild-type and kinase-dead SISOBIR1, and highly increased upon co-expression with P19. Co-agroinfiltrations of the affinity-tagged proteins were performed in $N$. benthamiana leaves at an $\mathrm{OD}_{600}$ of 1 for each construct. Leaves were harvested at $2 \mathrm{dpi}$, and subjected to IP using anti-GFP beads, followed by IB. The Rubisco band of the input shows equal loading. 
(A)

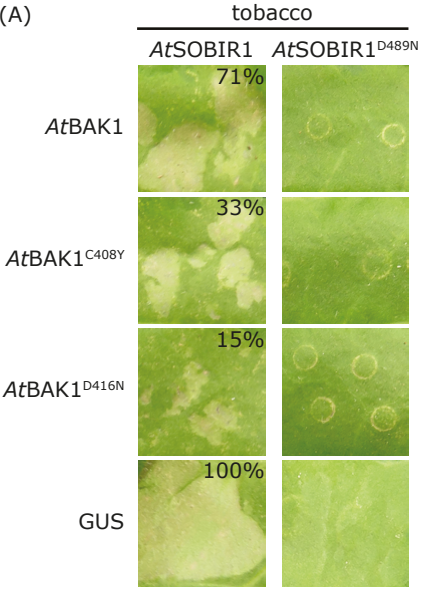

(C)

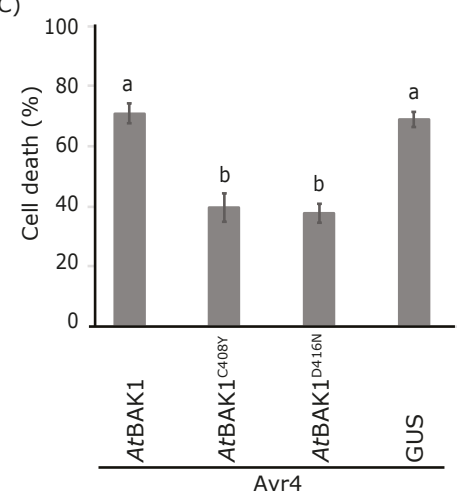

(B)

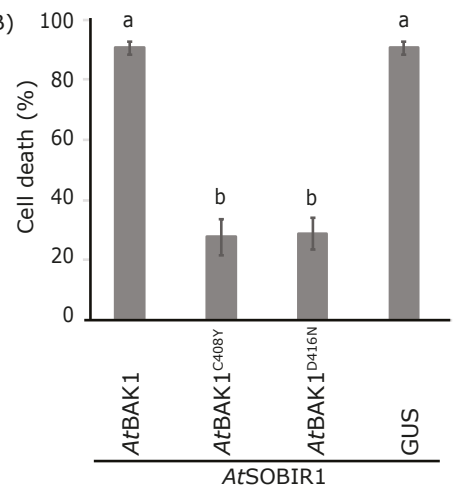

Figure S2. AtSOBIR1-mediated immunity is dependent on kinase-active BAK1. (A) Transient cOexpression of AtSOBIR1 in tobacco with AtBAK $1^{\mathrm{C} 408 \mathrm{Y}}$ or $A t \mathrm{BAK} 1^{\mathrm{D} 416 \mathrm{~N}}$ results in reduced AtSOBIR1 constitutive immune activity, as compared to co-expression of AtSOBIR1 with wild-type AtBAK1 or GUS. The indicated constructs were agroinfiltrated at an $\mathrm{OD}_{600}$ of 0.7 . Pictures were taken at $2 \mathrm{dpi}$, and are representative for agroinfiltration of eight leaves per sample. (B) Quantification of the percentage of cell death as shown in 4A. Percentages of constitutive cell death are represented as mean +/- SE. The letters indicate significant differences at $p<0.05$, as determined by one-way ANOVA, including a Tukey post hoc-test. (C) Quantification of the percentage of HR as shown in 4B. Percentages of Avr4-induced HR are represented as mean $+/$ - SE. The letters indicate significant differences at $p<0.05$, as determined by one-way ANOVA, including a Tukey post hoc-test. 



\section{Chapter 4}

\section{Phosphorylation of specific residues of the kinase domain of SOBIR1 is essential for its role in immune signalling}

Aranka M. van der Burgh'1, Jelle Postma², Hanna Rovenich ${ }^{1+}$, Sjef Boeren ${ }^{3}$, Silke Robatzek², Matthieu H. A. J. Joosten

\footnotetext{
${ }^{1}$ Laboratory of Phytopathology, Wageningen University, Droevendaalsesteeg 1, 6708 WE Wageningen, The Netherlands

${ }^{2}$ The Sainsbury Laboratory, Norwich Research Park, Norwich NR4 7UH, UK

${ }^{3}$ Laboratory of Biochemistry, Wageningen University, Strippeneng 4, 6708 PB Wageningen, The Netherlands

${ }^{+}$Current address: Botanical Institute, Cologne BiocenterUniversity of Cologne, Zülpicher Straße 47b, 50674 Cologne
} 


\section{Abstract}

Phosphorylation of signalling partners is an important molecular switch in various cellular processes, including the initiation of plant defence responses. The receptorlike kinase (RLK) Suppressor Of BIR1-1/Evershed (SOBIR1/EVR) is a key regulatory RLK that constitutively associates with receptor-like proteins (RLPs) to form bimolecular RLKs, and enables downstream signalling from the cell surface by RLPs that lack a kinase domain (KD) themselves. Trans-autophosphorylation of Arabidopsis thaliana (At) SOBIR1, and transphosphorylation by the co-receptor BRI1-Associated Kinase 1/ Somatic Embryogenesis Receptor Kinase 3 (BAK1/SERK3) has previously been shown to be essential for RLP-mediated immune signalling. Using mutational analyses, three dimensional modelling, and phosphoproteomics, we here show that AtSOBIR1 T519, T523, and T529 are all probable phosphorylation sites, and are likely to lock the SOBIR1-KD in the active conformation by controlling activation loop conformation, and its interaction with the Arg/Asp ('RD') catalytic motif. Phosphorylation on T522, Y532, although not conserved in tomato SOBIR1, and Y538 is likely to facilitate substrate specificity and differential affinity for interacting partners of the SOBIR1KD. Additionally, we show that AtSOBIR1, which constitutively activates immune responses when overexpressed in planta, is phosphorylated on several additional Thr and Ser residues of its KD. Specific phosphorylation of the KD of SOBIR1 likely enables this regulatory RLK to initiate immune signalling downstream of RLPS. 


\section{Introduction}

Plants haveevolveda sophisticated repertoire of receptorsto sense invading pathogens. Pathogens that invade the extracellular space can be sensed by transmembrane (TM)receptors that are present at the cell surface. These TM-receptors can be divided into two categories: receptor-like kinases (RLKs) and receptor-like proteins (RLPs). Both have an extracellular domain, which is often a leucine-rich repeat (LRR) domain, to enable pathogen recognition (Bohm et al., 2014; Zipfel, 2014). In contrast to RLKs, RLPs lack a cytoplasmic kinase domain (KD) for signalling. In RLKs, the KD forms a primary cytoplasmic switch to initiate signalling after pathogen recognition at the cell surface (Hohmann et al., 2017). RLPs constitutively interact with the regulatory RLK Suppressor Of BIR1-1/Evershed (SOBIR1/EVR, further referred to as SOBIR1), which provides a KD to enable cytoplasmic signalling (Liebrand et al., 2013 and 2014; Gust \& Felix, 2014; Chapter 3).

Ligand recognition is the first step in receptor-mediated defence. FlagellinSensing 2 (FLS2) and the EF-Tu Receptor (EFR) are well-studied RLKs from Arabidopsis thaliana ( $A t$, further referred to as Arabidopsis) that recognize the bacterial peptides flg22 and elf18, respectively (Chinchilla et al., 2007; Heese et al., 2007). Upon ligand recognition, the co-receptor BRI1-Associated Kinase 1/Somatic Embryogenesis Receptor Kinase 3 (BAK1/SERK3) (further referred to as BAK1) is recruited, after which defence responses are initiated (Chinchilla et al., 2007; Heese et al., 2007). RLPs, in a bimolecular complex with SOBIR1, also recruit BAK1 upon ligand recognition, which appears to be essential for signal initiation, similar to BAK1 recruitment to ligand-activated RLKs (Albert et al., 2015; Postma et al., 2016; Domazakis et al., 2018; Wang et al., 2018). The signal that is initiated from the cell surface eventually triggers a cascade of downstream responses to activate defence. These responses on the one hand consist of a fast response at the plasma membrane (PM), where the activated Respiratory Burst Oxidase Homologue D (RBOHD) enzyme mediates a swift production of reactive oxygen species (ROS) (Lu et al., 2010; Kadota et al., 2014; Li et al., 2014), whereas on the other hand, a cytoplasmic signalling cascade is triggered (Couto \& Zipfel., 2016). This cascade runs via the phosphorylation of mitogen activated protein kinases (MAPKs) and transcription factors that eventually mediate transcriptional reprogramming in the nucleus, involving defence gene activation (Lee et al., 2015; Zhang et al., 2018).

Phosphorylation of signalling partners is a well-known molecular switch in various cellular processes, including the initiation of plant defence and developmental responses (Afzal et al., 2008; Macho \& Zipfel, 2014; Macho et al., 2015). EFR, for example, is phosphorylated on a specific tyrosine (Tyr, Y) residue upon elf18 elicitation (Macho et al., 2014). A second example is the co-receptor BAK1, for which it has been shown that phosphorylation on several residues, including specific threonine (Thr, T) 
residues in the activation segment of its $K D$, is important for its role in immune- and brassinosteroid (BR) signalling (Yan et al., 2012; Schwessinger et al., 2011). A third example is Botrytis-Induced Kinase 1 (BIK1), which is a receptor-like cytoplasmic kinase (RLCK) that plays an essential role downstream of RLKs in immune signalling (Zhang et al., 2010; Lu et al., 2010). BIK1 is phosphorylated by BAK1 upon recognition of flg22 by FLS2, after which BIK1 activates RBOHD by phosphorylating this enzyme at specific sites (Lin et al., 2014; Kadota et al., 2014; Li et al., 2014). Interestingly, both BAK1 as well as BIK1 are not only involved in signalling for immunity, but also in BR signalling with the RLK BR-Insensitive 1 (BRI1) (Li et al., 2002; Nam \& Li, 2002; Lin et al., 2013). BRI1, on its turn, has also been shown to be phosphorylated upon elicitation by its ligand (BR), to enable growth and developmental responses (Wang et al., 2008; Oh et al., 2009; Bojar et al., 2014). In dual specificity kinases, the class to which plant kinases like BAK1, SOBIR1, BRI1, and BIK1 belong, phosphorylation can occur on Thr, serine (Ser, S), as well as on Tyr residues (Leslie et al., 2010; Oh et al., 2009; Lin et al., 2014; Xu et al., 2013). Differential phosphorylation of these kinases probably plays an essential role in passing on ligand-specific signals, resulting in a specific output either affecting defence activation or developmental processes (Macho \& Zipfel, 2014; Macho et al., 2015; Hohmann et al., 2017; Hohmann et al., 2018).

Kinases represent a large and diverse group of proteins, and their structure has been well-studied. Kinases consist of a small $\mathrm{N}$-lobe and a large C-lobe, and these two lobes form a cleft wherein an ATP molecule and the substrate to be phosphorylated fit (Kornev et al., 2006; Johnson, 2001). In the N-lobe a lysine (Lys, $\mathrm{K}$ )/glutamic acid (Glu, E) pair plays an important role in stabilisation of the active $K D$, whereas a conserved glycine-rich loop is important for ATP binding (Kornev et al., 2006; Kornev \& Taylor, 2010). Kinases can be classified into 'RD' and 'non-RD' kinases. This depends on whether an arginine residue (Arg, $R$ ) precedes the highly conserved catalytic aspartic acid (Asp, D) residue in the catalytic loop, located in the C-lobe (Johnson et al., 1996). The Asp residue in the RD motif is important to correctly orient the hydroxyl group of the substrate protein to be phosphorylated towards the bound ATP molecule, of which the gamma phosphate will be transferred (Kornev et al., 2006; Adams, 2001; Kornev \& Taylor, 2010). The activation segment, which also resides in the C-lobe, is an essential part of the KD and controls its activity (Johnson et al., 1996; Nolen et al., 2004). The most flexible and diverse part of the activation segment is the activation loop (Adams, 2003; Nolen et al., 2004). In contrast to non-RD kinases, RD kinases are known to require phosphorylation of one or more amino acid residues of their activation loop in order to become active (Johnson et al., 1996). After addition of a phosphate group to the so-called primary phosphorylation site, a particular amino acid residue present in the activation loop, the positively charged Arg residue of the RD motif interacts with this phosphorylated residue, thereby controlling the conformation and activation of the activation- and 
catalytic loop (Johnson et al., 1996; Adams, 2001; Nolen et al., 2004; Kornev \& Taylor, 2010). Additionally, phosphorylation on the activation loop confers specificity of the KD for the interaction with binding partners (Nolen et al., 2004). On the C-terminal flank of the activation loop the P+1 loop is located, which is important for interaction with, and specificity for, the substrate (Nolen et al., 2004; Kornev \& Taylor, 2010). The name ' $\mathrm{P}+1$ loop' refers to its interaction with the residue neighbouring the phosphorylation site ( $\mathrm{P}$-site) of the substrate. The activation loop and $\mathrm{P}+1$ loop are anchored by an $\mathrm{N}$-terminal and $\mathrm{C}$-terminal anchor. The $\mathrm{N}$-terminal anchor, with its conserved residues Asp, phenylalanine (Phe, P), and glycine (Gly, G), the so-called DFG motif, is involved in magnesium binding (Nolen et al., 2004). The C-terminal anchor, with the conserved residues alanine (Ala, A), proline (Pro, P), and Glu, the socalled APE motif, extends into the $\alpha E F / \alpha F$ loop and stabilizes the $P+1$ loop (Nolen et al., 2004; Kornev \& Taylor, 2010).

The RLK SOBIR1 was initially identified as a positive regulator of defence in Arabidopsis in a suppressor screen of BAK1-Interacting RLK 1 (BIR1), which is a negative regulator of defence (Gao et al., 2009). Later, SOBIR1 was identified as a constitutive interactor of RLPs, such as Cf-4 (Liebrand et al., 2013). Cf-4 recognizes the extracellular effector Avr4 that is secreted by Cladosporium fulvum, an apoplastic fungal leaf pathogen of Solanum lycopersicum (Sl, tomato) (de Wit et al., 1977). The KD of SOBIR1 has been shown to be a dual specificity RD kinase, as it is able to trans-autophosphorylate in vitro on Ser, Thr, and Tyr residues (Leslie et al., 2010). Recent in planta studies revealed a strong phosphorylation of AtSOBIR1, which constitutively signals for immunity, upon its overexpression in Nicotiana benthamiana (Nb) (Wu et al., 2018/Chapter 2; Chapter 3). Constitutive immune activity and high phosphorylation levels were shown to be positively correlated, as the kinasedead mutant AtSOBIR1D489N does neither show constitutive immune activity nor phosphorylation (Chapter 3). Additionally, a role of BAK1 as a transphosphorylation partner of SOBIR1 in immune signalling was shown and it is proposed that BAK1 transphosphorylates SOBIR1 to signal for immunity (Chapter 3).

Here we set out to analyse the role of phosphorylation of the SOBIR1-KD in immune signalling. We show that four conserved Thr residues in the activation segment of AtSOBIR1, namely T519, T522, T523, and T529, are required for immune signalling. Individual mutations of these residues to Ala reduce the constitutive immune activity of AtSOBIR1 in Nicotiana tabacum (tobacco) and $N$. benthamiana, and reduce its ability to function downstream of Avr4-activated Cf-4 in complementation essays. Additionally, three Tyr residues, Y436, Y532 (although not conserved in tomato SOBIR1), and Y538, of which Y436 is located in the $\mathrm{N}$-lobe, Y532 in the P+1 loop, and $Y 538$ in the $\alpha E F / \alpha F$ loop of the AtSOBIR1-KD, are also involved in immune signalling. Interestingly, phosphoproteomics detected the phosphorylation of T519 and several other Thr and Ser residues in the KD of constitutively immuno-active 
AtSOBIR1. Specific phosphorylation of specific amino acid residues present in the KD of SOBIR1, likely enables this regulatory RLK to specifically switch on immune signalling downstream of RLPs.

\section{Results}

\section{SOBIR1 contains a canonical KD with conserved potential phosphorylation sites}

The regulatory RLK SOBIR1 contains a canonical RD kinase, with a small $\mathrm{N}$-lobe and a large C-lobe (Fig. 1A and 1B). Further well-known motifs and segments, like the lysine/glutamic acid pair, the glycine-rich loop, the catalytic loop with the RD motif, and the activation segment, can be clearly recognised (Fig. 1A and 1B) (Nolen et al., 2004; Kornev et al., 2006). To identify potential phosphorylation sites in the KD of SOBIR1 that might be essential for its signalling role, we aligned the SOBIR1-KD sequences from 12 diverse plant species, using the KD of AtBAK1 as an outgroup (Fig. S1A and S1B). This alignment shows strong overall sequence conservation throughout the SOBIR1-KDs, as well as various similarities and dissimilarities to the AtBAK1-KD.

In the activation segment of $A t S O B I R 1$, one Ser and four Thr residues are located (Fig. $1 \mathrm{~A}$ and $\mathrm{S} 1 \mathrm{~B}$ ). These particular residues are almost completely conserved throughout all analysed SOBIR1-KD sequences (Fig. S1B). Furthermore, these five amino acids are $100 \%$ conserved across the SOBIR1-KDs of all sequenced Arabidopsis ecotypes (Weigel \& Mott, 2009). Tomato carries two SOBIR1 homologues, SISOBIR1 and SISOBIR1-like (Liebrand et al., 2013), and also here these five Thr/Ser amino acids are $100 \%$ conserved in these two SOBIR1 homologues across the different tomato species of which the genome sequence is known (Aflitos et al., 2014).

The involvement of Tyr phosphorylation in plant RLK signalling, in addition to the phosphorylation of Ser and Thr residues, has recently gained much attention (Oh et al., 2009 and 2011; Macho et al., 2014 and 2015). The AtSOBIR1-KD has eight Tyr residues (Fig. 1A and S1B), of which the positions are fully conserved across the various sequenced Arabidopsis ecotypes (Weigel \& Mott, 2009). Six of these Tyr residues are also conserved in SISOBIR1, and seven are conserved in SISOBIR1-like (Fig. S1B). Y532 (the numbering is based on AtSOBIR1) is not conserved in SISOBIR1, and Y617 is not conserved both in SISOBIR1 and SISOBIR1-like (Fig. S1B). The Tyr residues in the KD sequences of SISOBIR1 and SISOBIR1-like across different tomato species are again highly conserved (Aflitos et al., 2014). 
A

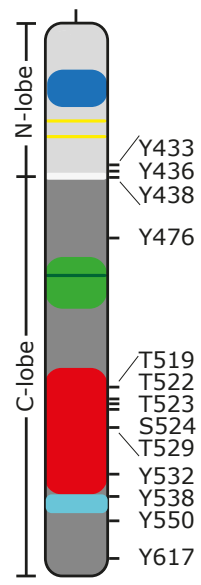

Gly-rich loop

Lys/Glu pair

Catalytic loop

Catalytic Asp

Activation segment

aEF/aF loop
B

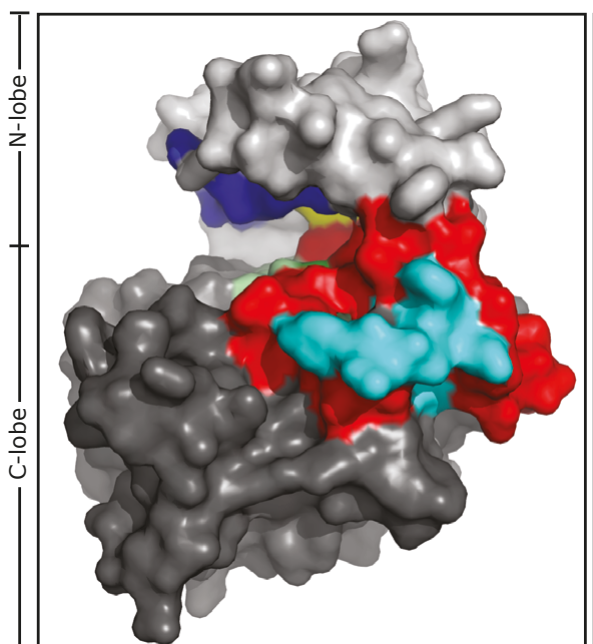

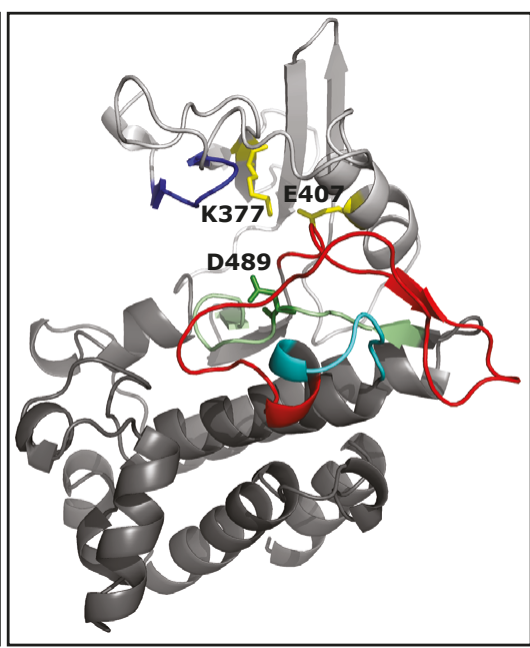

Figure 1. SOBIR1 contains a canonical kinase domain with conserved potential phosphorylation sites. (A) Schematic overview of the kinase domain (KD) of AtSOBIR1 with its various motifs and domains. Amino acids that are potential phosphorylation sites are indicated. See also Fig. S1B. (B) A three dimensional model of the structure of AtSOBIR1-KD, based on the crystal structure of BRI1 (Bojar et al., 2014). For the various domains, the same colour code as employed in $A$ is used. The model is depicted as surface (left), and as a ribbon (right) to visualise the inside of the KD.

These sequence analyses show that the KD of SOBIR1 contains several highly conserved and thereby potentially essential Tyr residues, and Thr/Ser residues in the activation segment, which might be differentially phosphorylated upon ligand-based activation of the SOBIR1-associated RLP. 


\section{The threonine residues present in the activation segment of SOBIR1 are essential for its role in signalling for immunity}

As mentioned above, there are four Thr residues and one Ser residue present in the activation segment of the KD of SOBIR1 and their strong conservation led us to hypothesise that these residues might be important for the signalling function of SOBIR1 (Fig. S1B). We therefore set out to investigate the role of these residues in immune signalling by SOBIR1, using a mutational analysis. As the overall amino acid sequences of the KDs of AtSOBIR1 and SISOBIR1 are also strongly conserved, we selected to use AtSOBIR1 for mutational analysis. AtSOBIR1 constitutively activates immune responses upon its overexpression in tobacco and $N$. benthamiana, which can be observed as cell death and MAPK activation (Wu et al., 2018/Chapter 2). Furthermore, AtSOBIR1 functions downstream of Cf-4 in complementation assays (Liebrand et al., 2013). These characteristics enable us to screen mutants of AtSOBIR1 for functionality by determining their possible loss of constitutive immune activity, and their altered capacity to restore the Cf-4/Avr4-triggered cell death (the hypersensitive response $(\mathrm{HR})$ ) in complementation assays in SOBIR1-silenced $N$. benthamiana:Cf-4 plants.

We generated five AtSOBIR1 mutants, each with one of the Thr/Ser residues in the activation segment substituted by an Ala residue (Fig. 1A and S1B). Upon overexpression in tobacco and $N$. benthamiana (in the latter co-infiltrated with the silencing suppressor P19), all five AtSOBIR1 mutants showed reduced constitutive immune activity compared to wild-type, as visualised by a suppressed cell death response (Fig. 2A, S2A and S2B). AtSOBIR1 ${ }^{\mathrm{TS} 19 \mathrm{~A}}$ and AtSOBIR1 ${ }^{\mathrm{TS} 29 \mathrm{~A}}$ were strongly reduced in constitutive immune activity, AtSOBIR1 $1^{\text {T522A }}$ and AtSOBIR1 $1^{\text {T523A }}$ were mildly reduced in constitutive immune activity, and AtSOBIR $1^{\text {S524A }}$ was only slightly reduced in its potential to cause cell death. All five mutants did accumulate upon their overexpression in tobacco and N. benthamiana (Fig. S2C and S2D), and their accumulation levels showed a trend of reverse correlation with their level of constitutive immune activity, although this was not statistically significant (Fig. S2D) (Wu et al., 2018/Chapter 2). Although the five studied amino acids do not seem to be equally important for AtSOBIR1-triggered cell death, MAPK activation was virtually absent for all five mutants (Fig. 2B), which indicates that all five amino acids do play a role in immune signalling downstream of AtSOBIR1.

Phosphorylation of SOBIR1 depends on its own kinase activity and on that of BAK1. Trans-autophosphorylation of SOBIR1 and transphosphorylation with BAK1 are thought to be essential to activate downstream immune responses (Chapter 3 ). To determine the effect of the mutation of the Thr/Ser residues in the activation segment of AtSOBIR1 on its overall phosphorylation status, we performed a Pro-Q staining on the in planta produced mutant proteins. This showed that AtSOBIR1 $1^{\text {T519A }}$ and AtSOBIR1 ${ }^{\mathrm{T} 529 \mathrm{~A}}$, the mutants that are strongly reduced in their constitutive 
immune activity, also show strongly reduced overall phosphorylation level, similar to the kinase-dead AtSOBIR1 $1^{\mathrm{D} 489 \mathrm{~N}}$ mutant (Fig. $2 \mathrm{C}$ and S3). Therefore, we conclude that T519 and T529 are important for the overall kinase activity of SOBIR1. AtSOBIR1 $1^{\text {S524A }}$ was not reduced in its overall phosphorylation level, which correlates with its partially maintained constitutive immune activity (Fig. 2A). Interestingly, the overall phosphorylation levels of AtSOBIR $1^{\mathrm{T522A}}$ and AtSOBIR1 $1^{\mathrm{T523A}}$, which are mutants that are only mildly reduced in their constitutive immune activity, were similar to wildtype AtSOBIR1. This suggests that T522 and T523 do not play a role in overall SOBIR1 kinase activity, but their phosphorylation likely aids in signalling for immunity.

The induction of constitutive immune activity by AtSOBIR1 likely reflects the ability of SOBIR1 to signal downstream of RLPs, as both the constitutive activity of AtSOBIR1 and the activation of the Cf-4/SOBIR1 complex by Avr4 typically result in clear immune responses such as MAPK activation and cell death (Wu et al., 2018/ Chapter 2). To further validate the role of the putative Thr/Ser phosphorylation sites in the activation segment of SOBIR1 in immune signalling by RLPS, we analysed the ability of the mutants of AtSOBIR1 to signal downstream of $\mathrm{Cf}-4$. For this, we employed heterologous complementation of endogenous NbSOBIR1 and NbSOBIR1-like by AtSOBIR1. Previously, it has been shown that eGFP-tagged wild-type AtSOBIR1, but not kinase-dead AtSOBIR1 ${ }^{\mathrm{D} 489 \mathrm{~N}}$, complements the compromised Cf-4/Avr4-triggered HR in NbSOBIR1(/-like)-silenced N. benthamiana:Cf-4 plants (Liebrand et al., 2013). We found that all five mutants of AtSOBIR1 are significantly hampered in their ability to function downstream of Cf-4 in mediating cell death, when transiently expressed in combination with Avr4, in the absence of P19 (Fig. 2D and S4A). The observed cell death was the result of a Cf-4/Avr4-specific HR, and was not caused by AtSOBIR1induced constitutive immunity, as co-expression of the different AtSOBIR1 variants with Avr9 does not lead to cell death (Fig. 2D). Mutation of T529 to Ala leads to the same phenotype as kinase-dead AtSOBIR1 ${ }^{\text {D489N }}$, which corresponds with the loss of constitutive immune activity and the loss of overall phosphorylation of this mutant (Fig. 2A and C). AtSOBIR $1^{\mathrm{S} 524 \mathrm{~A}}$ is only mildly reduced in its ability to signal for Cf-4/Avr4$\mathrm{HR}$, which is as expected, as also its constitutive immune activity and phosphorylation are only mildly reduced (Fig. 2A and C). The Ala substitutions of T519, T522, and T523 clearly affect AtSOBIR1 functioning downstream of Cf-4/Avr4, but not as strong as $A t S O B I R 1^{\text {D489N. }}$. As AtSOBIR1 $1^{\text {T522A }}$ and AtSOBIR1 $1^{\text {T523A }}$ retained an observable level of overall phosphorylation, similar to AtSOBIR1 wild-type (Fig. 2C), it is likely that specific phosphorylation of these two Thr residues influences immune signalling of SOBIR1. The mutations do not affect the ability of AtSOBIR1 to interact with Cf-4, as for all AtSOBIR1 mutants an interaction with Cf-4 was observed (Fig. S4B). 

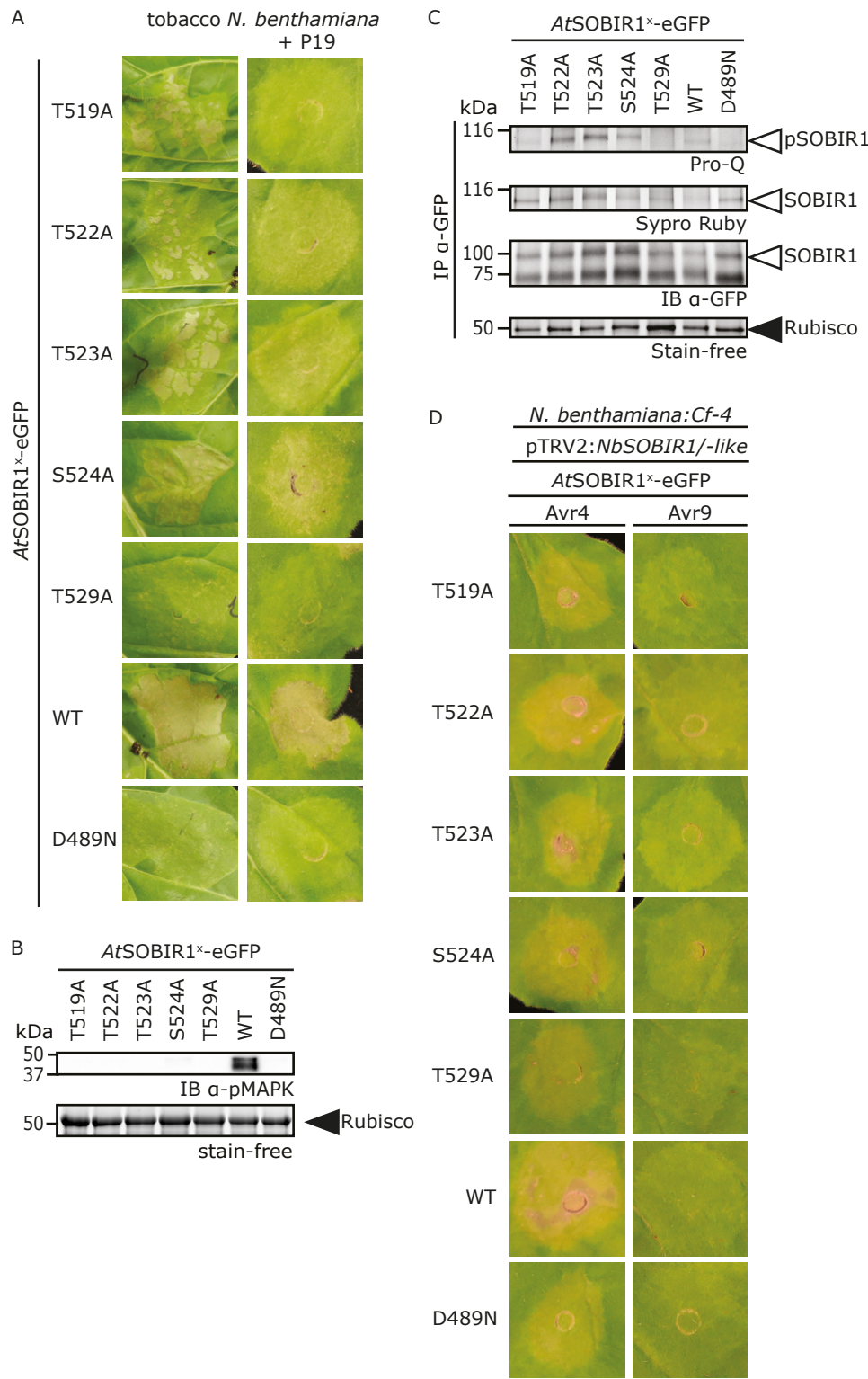

Figure 2. The threonine residues in the activation segment of the KD of SOBIR1 are essential for its role in signalling for immunity. (A) AtSOBIR $1^{T 519 A}$ and $A t S O B I R 1^{T 529 A}$ are strongly reduced in their constitutive immune activity, whereas AtSOBIR $1^{\mathrm{T} 522 \mathrm{~A}}$ and $A t S O B I R 1^{1523 \mathrm{~A}}$ show an intermediate reduction, and $A t S O B I R 1^{5524 A}$ is only mildly reduced in its constitutive immune activity. Leaf sectors of tobacco (left) and $N$. benthamiana (right) were transiently transformed with the various eGFP-tagged AtSOBIR1 mutants, and AtSOBIR1 wild-type (WT) or kinase-dead (D489N) as positive and negative control, respectively, at an $\mathrm{OD}_{600}$ of 1 . In N. benthamiana, $\mathrm{P} 19$ was co-infiltrated at an $\mathrm{OD}_{600}$ of 1 . Pictures were taken at 3 days post infiltration (dpi). Representative pictures of at least three independent experiments are shown. See also Fig. S2. (B) MAPKs are not activated upon overexpression of any of the five AtSOBIR1 activation segment mutants. The same AtSOBIR1 constructs as analysed in panel A, were transiently co-expressed with P19 
in $N$. benthamiana, both at an $\mathrm{OD}_{600}$ of 1 . About $40 \mathrm{~h}$ after agroinfiltration, before cell death induced by wild-type AtSOBIR1 became apparent, total protein was extracted and analysed for MAPK activation using anti-p42/p44-erk antibody. The Rubisco band in the stain-free gel indicates equal loading. The experiment was performed three times, with similar results. (C) Pro-Q phosphoprotein staining of in planta produced immunoprecipitated eGFP-tagged AtSOBIR1 mutants shows strong reduction in the overall phosphorylation level of $A t S O B I R 1^{T 529 A}$ and the kinase-dead control AtSOBIR1 $1^{\text {D489N }}$. Note that AtSOBIR1 $1^{\text {T519A }}$ is also significantly reduced in its overall phosphorylation. The phosphorylation level of AtSOBIR1 $1^{\text {T522A, }}$ AtSOBIR1 $1^{T 523 A}$, and AtSOBIR1 $1^{5524 A}$ is not significantly different from wild-type AtSOBIR1. The various AtSOBIR1-eGFP mutants were transiently co-expressed with $\mathrm{P} 19$, all at an $\mathrm{OD}_{600}$ of 1 , and about $40 \mathrm{~h}$ after agroinfiltration leaves were harvested and subjected to IP. Sypro Ruby stain and IB analysis using anti-GFP antibody show the total amounts of AtSOBIR1 protein in the IP samples. The experiment was performed three times, and a representative experiment is shown. The Rubisco background band in the stain-free gel indicates equal loading. See also Fig. S3. (D) All four Thr residues and the Ser residue in the activation segment of SOBIR1 are required for full SOBIR1 function downstream of Cf-4. The eGFP-tagged AtSOBIR1 Thr and Ser mutants were transiently co-expressed (at an $\mathrm{OD}_{600}$ of 0.7), with Avr4 or Avr9, both at an $\mathrm{OD}_{600}$ of 0.03 , in leaves of $N$. benthamiana:Cf-4 plants that, two to three weeks earlier, had been inoculated with pTRV2:NbSOBIR1/-like to initiate VIGS of endogenous NbSOBIR1. Note that AtSOBIR1 $1^{\mathrm{TS} 29 \mathrm{~A}}$

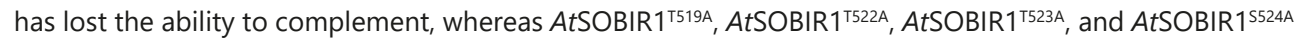
are compromised in their ability to complement for the loss of endogenous NbSOBIR1. The hypersensitive response (HR) was scored in a randomized, blind way. Pictures were taken at $3 \mathrm{dpi}$ and are representative for at least three independent experiments. See also Fig. S4.

In conclusion, these experiments show that the four Thr phosphorylation sites in the activation segment of SOBIR1 play an important role in immune signalling by SOBIR1, whereas the Ser residue appears to play a less important role in this process.

\section{Two conserved tyrosine residues in the KD of SOBIR1 are essential for its role in signalling for immunity}

To investigate the role of Tyr residues in the KD of SOBIR1 in immune signalling, and the importance of their possible phosphorylation, we generated eight eGFPtagged AtSOBIR1 mutants, each with one individual Tyr residue replaced by a phenylalanine (Phe, F) (Fig. 1A, S1B). Phe has a structure similar to Tyr, but lacks the hydroxyl group at the 6-carbon aromatic ring, which means that Phe cannot be phosphorylated. Screening for constitutive immune activity of these SOBIR1 mutants in tobacco and $N$. benthamiana revealed that AtSOBIR $1^{\mathrm{Y} 433 \mathrm{~F}}$, AtSOBIR $1^{\mathrm{Y} 476 \mathrm{~F}}$, AtSOBIR1 $1^{\mathrm{Y} 550 \mathrm{~F}}$, and AtSOBIR1 ${ }^{\mathrm{Y} 617 \mathrm{~F}}$ all exhibit a level of constitutive immune activity similar to wild-type AtSOBIR1 (Fig. 3A, S5A and S5B). By contrast, AtSOBIR1 ${ }^{\mathrm{Y} 438 \mathrm{~F}}$ was only mildly compromised, whereas AtSOBIR $1^{\mathrm{Y} 436 \mathrm{~F}}$ and AtSOBIR $1^{\mathrm{Y} 538 \mathrm{~F}}$ were significantly compromised in their constitutive immune activity. Interestingly, the AtSOBIR1 ${ }^{\mathrm{Y} 532 \mathrm{~F}}$ mutant showed increased constitutive immune activity, as the cell death response that it provokes appears to be stronger and faster when compared to wild-type AtSOBIR1 (Fig. S5C). 

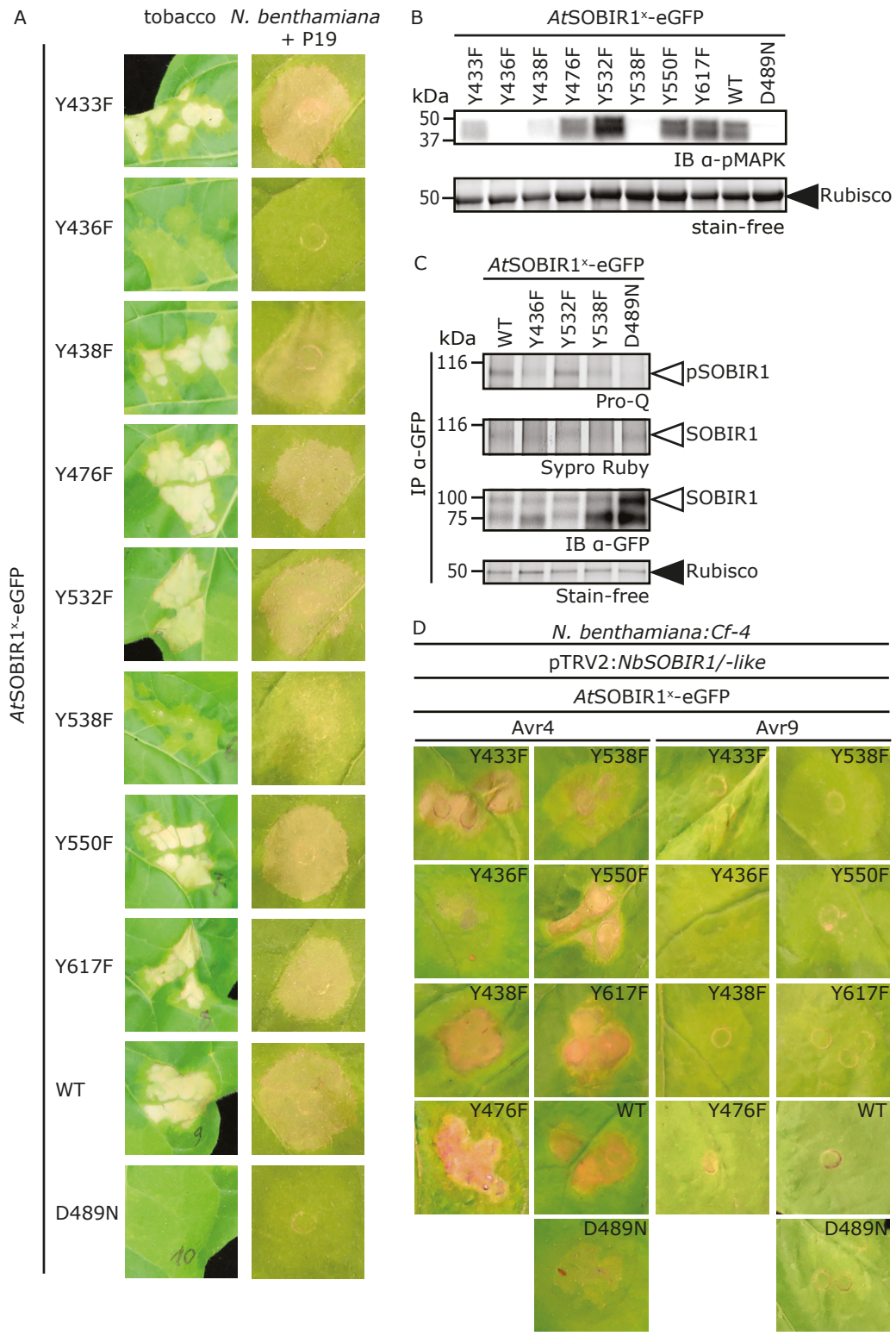

Figure 3. Two conserved tyrosine residues in the KD of SOBIR1 are essential for its role in immunity. (A) Two Tyr residues in the KD of AtSOBIR1 are essential for its constitutive immune activity in tobacco (left) and $N$. benthamiana (right). AtSOBIR1 constitutive immunity is lost for the two Tyr mutants AtSOBIR $1^{\text {Y436F }}$ and AtSOBIR1 $1^{\text {Y538F. }}$. Leaf sectors were transiently transformed with the eight AtSOBIR1 Tyr mutants, AtSOBIR1 wild-type (WT) and kinase-dead AtSOBIR1 ${ }^{D 489 \mathrm{~N}}$ that were all fused to eGFP, at an $\mathrm{OD}_{600}$ of 1. In N. benthamiana, P19 was co-infiltrated at an $\mathrm{OD}_{600}$ of 1 . Pictures were taken at $3 \mathrm{dpi}$. Representative pictures of at least three independent replicates are shown. See also Fig. S5. (B) MAPKs are not activated

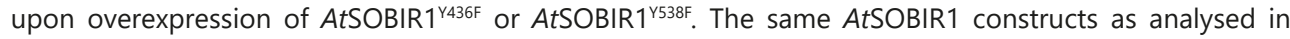
panel A, were transiently co-expressed with P19 in N. benthamiana, all at an $\mathrm{OD}_{600}$ of 1 . About $40 \mathrm{~h}$ after 
agroinfiltration, before cell death induced by wild-type AtSOBIR1 became apparent, total protein was extracted and analysed for MAPK activation using anti-p42/p44-erk antibody. The intensity of the Rubisco band in the stain-free gel indicates equal loading. The experiment was performed three times, with similar results. (C) Pro-Q phosphoprotein staining of in planta produced immunoprecipitated eGFP-tagged AtSOBIR1 Tyr mutants shows reduced overall phosphorylation levels of AtSOBIR1 $1^{\mathrm{Y} 336 \mathrm{~F}}$ and AtSOBIR $1^{\mathrm{YS3} 38 \mathrm{~F}}$. The indicated AtSOBIR1-eGFP constructs were transiently co-expressed with P19, all at an $\mathrm{OD}_{600}$ of 1 , and about 40h after agroinfiltration leaves were harvested and subjected to IP. Sypro Ruby stain and IB analysis using anti-GFP antibody show the total amounts of AtSOBIR1 protein in the IP samples. The experiment was performed three times and a representative experiment is shown. See also Fig. S6. (D) Y436 and Y538 are required for full SOBIR1 function downstream of Cf-4. The eGFP-tagged AtSOBIR1 Tyr mutants were transiently co-expressed (at an $\mathrm{OD}_{600}$ of 0.7 ), with Avr4 or Avr9, both at an $\mathrm{OD}_{600}$ of 0.03 , in $N$. benthamiana:Cf-4 plants that, two to three weeks earlier, had been inoculated with pTRV2:NbSOBIR1/like to initiate VIGS of endogenous NbSOBIR 1 . Note that the lack of an HR indicates that AtSOBIR1 $1^{\text {Y436F }}$ has lost the ability to complement the silencing of endogenous NbSOBIR1, whereas AtSOBIR1 $1^{\mathrm{Y} 538 \mathrm{~F}}$ is strongly compromised in its ability to do so. The HR was scored in a randomized, blind way. Pictures were taken at $3 \mathrm{dpi}$ and are representative for at least three independent experiments. See also Fig. S7.

The level of induction of constitutive immunity by the AtSOBIR1 Tyr to Phe mutants positively correlates with MAPK activation, as the AtSOBIR1 Tyr mutants that are able to induce cell death, also trigger MAPK phosphorylation (Fig. 3B). Accordingly, AtSOBIR $1^{\mathrm{r} 436 \mathrm{~F}}$ and $A t \mathrm{SOBIR} 1^{\mathrm{Y} 538 \mathrm{~F}}$ that do not trigger cell death, and $A t \mathrm{SOBIR} 1^{\mathrm{r} 438 \mathrm{~F}}$, which has mildly compromised activity, showed strongly reduced levels of MAPK activation (Fig. 3B). In addition, AtSOBIR1 $1^{\mathrm{Y} 532 \mathrm{~F}}$, the mutant with increased constitutive immune activity, shows elevated MAPK activation (Fig. 3B). Pull-down of the various eGFPtagged AtSOBIR1 mutants from agroinfiltrated leaves of $N$. benthamiana, followed by immunoblot analysis, demonstrated proper accumulation of the eight Tyr mutants, as well as wild-type AtSOBIR1 and kinase-dead AtSOBIR1 ${ }^{\text {D489N }}$ (Fig. S5D and S5E). The accumulation levels of the AtSOBIR1 Tyr to Phe mutant proteins inversely correlated with their constitutive immune activity (Wu et al., 2018 / Chapter 2), as was earlier observed as a clear trend for the Thr and Ser mutants in the activation segment of AtSOBIR1 (Fig. S2C and S2D). AtSOBIR1 $1^{\mathrm{Y} 436 \mathrm{~F}}$ and AtSOBIR1 $1^{\mathrm{Y} 538 \mathrm{~F}}$, which are strongly suppressed in their constitutive immune activity, accumulate to relatively high levels when compared to wild-type AtSOBIR1, whereas kinase-dead AtSOBIR1 ${ }^{\text {D489N }}$ showed the highest accumulation levels (Fig. S5D and S5E). By contrast, AtSOBIR1 ${ }^{\text {Y532F, }}$ exhibiting enhanced constitutive immune activity, accumulates at much lower levels than wild-type AtSOBIR1. The other five AtSOBIR1 Tyr mutants showed accumulation levels similar to wild-type AtSOBIR1, indicating again that these proteins still have the capacity to activate immune signalling (Fig. 3B, S5D and S5E).

SOBIR1 localizes at the plasma membrane (PM) and in endosomes, and forms complexes with RLPs at the PM (Leslie et al., 2010; Liebrand et al., 2013; Albert et al., 2015; Postma et al., 2016). To rule out a possible effect of the Tyr to Phe mutations on protein localization, we analysed their subcellular localization by confocal microscopy. All AtSOBIR1 Tyr to Phe mutants were found to localize at the PM and cytoplasmic vesicles, similar to the localization of wild-type AtSOBIR1-eGFP (Fig. S5F). 
To address whether the Tyr mutations that affect AtSOBIR1 constitutive immune activity alter the overall phosphorylation status of the protein, we transiently expressed AtSOBIR1 ${ }^{\mathrm{Y} 436 \mathrm{~F}}$, AtSOBIR $1^{\mathrm{Y5} 32 \mathrm{~F}}$, and AtSOBIR $1^{\mathrm{Y5} 38 \mathrm{~F}}$, wild-type AtSOBIR1, and kinase-dead AtSOBIR1 ${ }^{1489 N}$ in $N$. benthamiana, together with $\mathrm{P} 19$. After immunoprecipitation, the phosphorylation status of the AtSOBIR1 variants was analysed by Pro-Q staining. This revealed a reduction in overall phosphorylation level of $A t S O B I R 1^{Y 436 F}$, a mutant that shows strongly suppressed constitutive immune activity (Fig. $3 \mathrm{C}$ and S6). The phosphorylation status of AtSOBIR $1^{\text {Y538F, }}$, which also exhibits compromised constitutive immune activity, was only mildly reduced when compared to wild-type AtSOBIR1 (Fig. 3C and S6). AtSOBIR1 $1^{\text {Y532F }}$ appeared to be phosphorylated at a similar level as wild-type AtSOBIR1 (Fig. 3C and S6).

We next addressed whether the Tyr residues that are required for the constitutive immune activity of $A t S O B I R 1$ also play a role in SOBIR 1 immune signalling downstream of $\mathrm{Cf}-4$. For this, we checked whether the Tyr to Phe mutants have the capacity to complement in NbSOBIRT(/-like)-silenced N. benthamiana:Cf-4, similar as described above for the Thr and Ser mutants. Due to its strong constitutive activity, AtSOBIR1 ${ }^{\mathrm{rS} 32 \mathrm{~F}}$ was not included in this experiment. We found that Y436 of AtSOBIR1 is crucial for $\mathrm{Cf}-4$-mediated HR, as transient expression of AtSOBIR1 ${ }^{\mathrm{Y} 436 \mathrm{~F}}$ in N. benthamiana:Cf-4 leaves, previously inoculated with pTRV2:NbSOBIRT(/-like), failed to support Cf-4mediated HR upon co-expression with Avr4 (Fig. 3D and S7A). AtSOBIR1 ${ }^{\text {Y538F }}$ was severely compromised in restoring the $\mathrm{Cf}-4$-mediated $\mathrm{HR}$ upon co-expression with Avr4, as the HR was highly suppressed when compared to complementing with wildtype AtSOBIR1. Both AtSOBIR1 $1^{\text {Y436F }}$ and AtSOBIR1 $1^{\text {Y538F }}$ normally interact with $\mathrm{Cf}-4$, which is therefore not explaining their lack of complementation (Fig. S7B). All five Tyr mutants of AtSOBIR1 that showed constitutive immune activity similar to wild-type AtSOBIR1

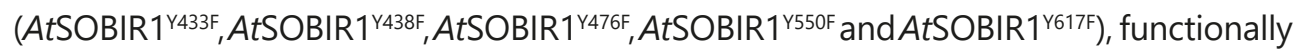
complemented the knockdown of endogenous NbSOBIR1 (Fig. 3D and S7A).

Taken together, our findings demonstrate that the conserved residues $Y 436, Y 532$, and $\mathrm{Y} 538$ are important for immune signalling by SOBIR1. Y436 and Y538 appear to be critical for a functional KD of AtSOBIR1, whereas Y532 appears to be required for the efficiency by which SOBIR1 signals for immunity, as the T523F mutation results in a gain-of-function protein. 


\section{A three dimensional model of the KD of AtSOBIR1 indicates the possible roles of its Thr and Tyr potential phosphorylation sites}

To determine the intrinsic roles of the important potential phosphorylation sites of SOBIR1 that were identified with the mutation analyses, we visualised their positions in a three dimensional (3D) model of the AtSOBIR1-KD (Fig. 4). A 3D surface visualization of the AtSOBIR1-KD model shows that T519, T522, S524, and Y538 all point outwards of the KD (Fig. 4A). The outward orientation suggests that phosphorylation of these residues might play a role in the affinity of the KD for interacting proteins (Nolen et al., 2004). T519, T522, and S524 are all located in the activation loop, but S524 seems to play a less important role in signalling by SOBIR1 than T519 and T522, because the S524 to Ala mutation did not completely abolish the constitutive immune activity of AtSOBIR1 (Fig. S1B and Fig. 2). In the 3D model, S524 is partly covered by the $\mathrm{N}$-lobe, making it less likely for $\mathrm{S} 524$ to play a role in potential interactions with other proteins (Fig. 4A). In its turn, Y538 is located in the $\alpha E F / \alpha F$ loop, just adjacent to the C-terminal anchor of the activation segment (Fig. 4A and S1B). The $\alpha E F / \alpha F$ loop stabilizes the activation loop, which folds on the $\alpha E F / \alpha F$ loop (Nolen et al., 2004). So, Y538 might contribute to stabilize the active conformation of the SOBIR1-KD.

A 3D ribbon visualization of the AtSOBIR1-KD model shows that T523, T529, and Y532, all point inwards into the direction of the catalytic site of the KD (Fig. 4B). T523, located in the activation loop, is in close proximity to the R488 residue, preceding the catalytic Asp in the RD motif of the catalytic loop (Fig. S1B). Phosphorylation of T523 is likely to be involved in stabilising the active conformation of the kinase, by interacting with the positively charged Arg residue (Kornev et al., 2006; Nolen et al., 2004). T529 is located in the P+1 loop, and in the 3D model it is visible that T529 is in close proximity to the catalytic Asp (D489) (Fig. 4B). Y532, which is also located in the $P+1$ loop, is oriented to the substrate-binding pocket of the SOBIR1-KD (Fig. 4B). As the Y523 to Phe mutation results in a gain-of-function (Fig. S5C), it is likely that the hydroxyl group and/or phosphorylation of this residue might inhibit substrate binding.

A 3D ribbon visualization of the AtSOBIR1-KD model shows that $\mathrm{Y} 436$ is in close proximity to the lysine/glutamic acid pair (K377/E407) that plays an important role in stabilisation of the active KD (Fig. 4C). The polar contact between the positively charged residue $\mathrm{K} 377$ and negatively charged residue E407 is essential for the stabilisation of the KD in the active state, whereas K377 also interacts with the negatively charged $\alpha$ and $\beta$ phosphates of the incorporated ATP molecule (Kornev et al., 2006). The $\mathrm{OH}$-group of SOBIR $1^{\mathrm{Y} 436}$ or its phosphorylation is likely to be essential to stabilize this K-E bridge, and to keep the KD in the active conformation. 

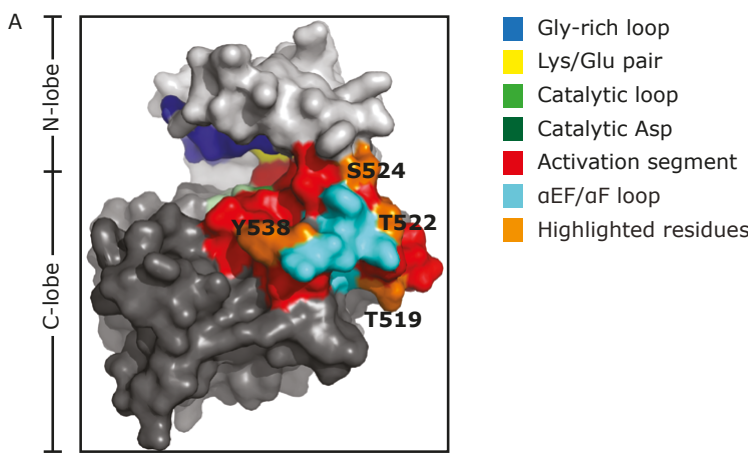

B

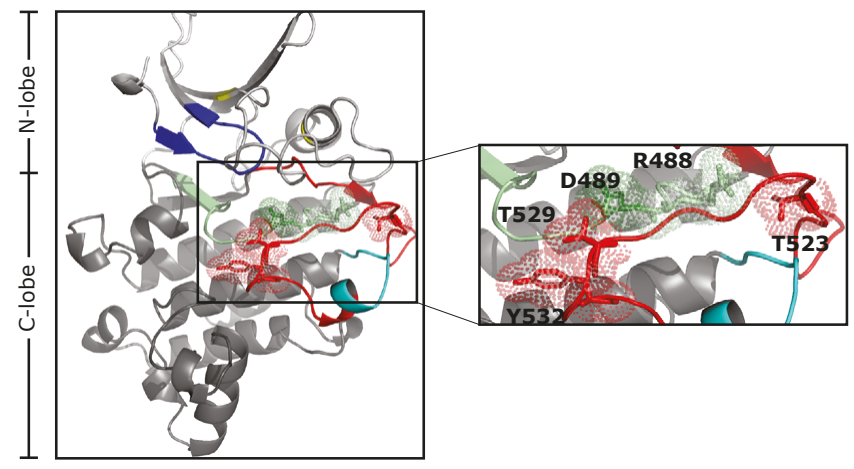

C

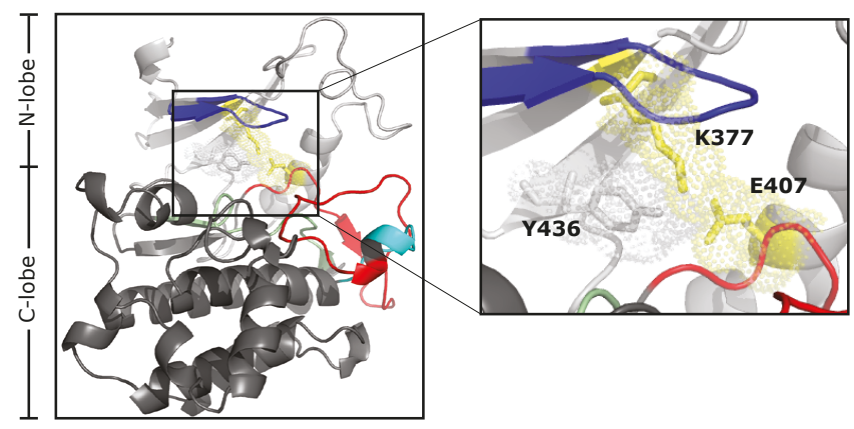

Figure 4. A three dimensional model of the KD of AtSOBIR1 indicates the possible roles of the Thr, Ser and Tyr potential phosphorylation sites. (A) Surface visualization of the AtSOBIR1-KD, where T519, T522, S524, and Y538 (highlighted in orange) are clearly oriented outwards. These residues, and likely their phosphorylation, might determine the interaction of the SOBIR1-KD with potential signalling partners. T519, T522, and S524 are located in the activation loop of the activation segment (red), whereas Y538 is located just outside the activation segment, likely stabilizing the active conformation of the activation segment. (B) Ribbon visualization of the AtSOBIR1-KD model (left), with detailed picture (right), shows that the residues T523, T529, and Y532 are oriented inwards. T523 is in close proximity to R488, and T529 is in close proximity to the catalytic D489. Y532 points into the substrate-binding pocket. (C) Ribbon visualization of the AtSOBIR1-KD model (left), with a detailed picture (right), shows that residue Y436 is in close proximity to the Lys (K) / Glu (E) pair (yellow). Y436, and possibly its phosphorylation, is important for stabilising the active conformation of the kinase. 
The other five highly conserved tyrosine residues that we selected for the mutational analyses, Y433, Y438, Y476, Y550, and Y617, which did not seem to play an important role in the function of SOBIR1 in immunity (Fig. 3), all appear not to be located at important positions of the KD (Fig. S8). The two tyrosine residues close to $\mathrm{Y} 436$, namely $\mathrm{Y} 433$ and $\mathrm{Y} 438$, are located in the $\mathrm{N}$-lobe and the hinge region between the $\mathrm{N}$ - and the C-lobe, respectively (Fig. S1B, and Fig. S8). Y476, Y550, and Y617 are located in the N-lobe (Fig. S1B, Fig. S8).

In conclusion, the 3D model of the AtSOBIR1-KD enables us to visualize that T519, T522, S524, and Y538 are exposed on the outside of the KD and are likely to be involved in the interaction of the SOBIR1-KD with signalling partners. Additionally, the model shows that T523, T529, and Y532 are oriented inwards. T523 and T529 possibly aid in the interaction with the RD motif, and Y532 in the interaction with the substrate to be phosphorylated. Furthermore, Y436 is probably involved in keeping the active kinase conformation by its interaction with the Lys/Glu pair.

\section{Differential phosphorylation of SOBIR1 downstream of Avr4 ligand-activated $\mathrm{Cf}-4$, as compared to the resting state, could not be shown}

To examine whether the potential phosphorylation sites of SOBIR1 that were found to be important for its role in immune signalling, are differentially phosphorylated in planta upon ligand recognition by the RLP that is associated with SOBIR1, we set out to analyse the phosphorylation status of SOBIR 1 downstream of $\mathrm{Cf}-4$ in the presence or absence of the ligand Avr4. First, we examined the overall phosphorylation level of the pool of SOBIR1 that is associated with $\mathrm{Cf}-4$, with and without treatment with Avr4. For this, we transiently expressed Myc-tagged AtSOBIR1 and SISOBIR1, and their kinase-dead variants as negative controls, in transgenic $N$. benthamiana:Cf-4-eGFP plants. After two days, we infiltrated the effector protein Avr4, or water as negative control, and harvested the leaves at 25 min after infiltration. Pull down of Cf-4-eGFP using GFP affinity beads, followed by Pro-Q stain and immuno-blot (IB) analysis of the pool of SOBIR1 co-precipitating with $\mathrm{Cf}-4$, showed that this pool of AtSOBIR1 is clearly phosphorylated (Fig. 5A). This corresponds with the clear phosphorylation of AtSOBIR1-eGFP when overexpressed in planta and immunoprecipitated directly (Chapter 3). However, the phosphorylation level of AtSOBIR1 appeared unchanged in the sample in which Cf-4 was elicited with Avr4, as compared to the sample where water was infiltrated (Fig. 5A). For SISOBIR1 no distinct Pro-Q signal was visible for either the Avr4- or the water-infiltrated sample (Fig. 5A). 


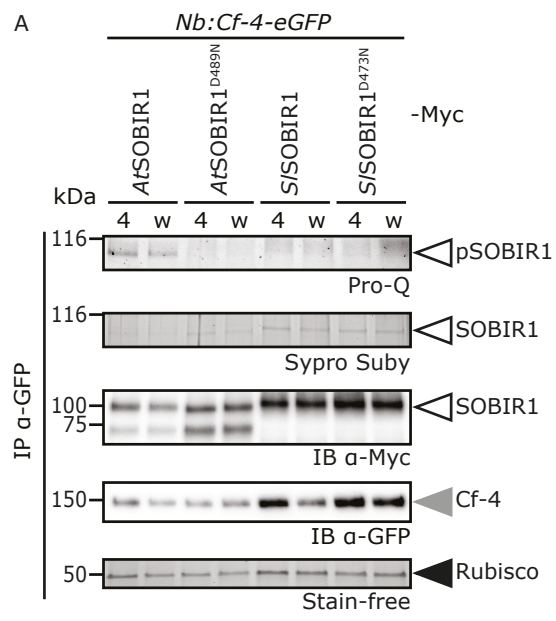

B

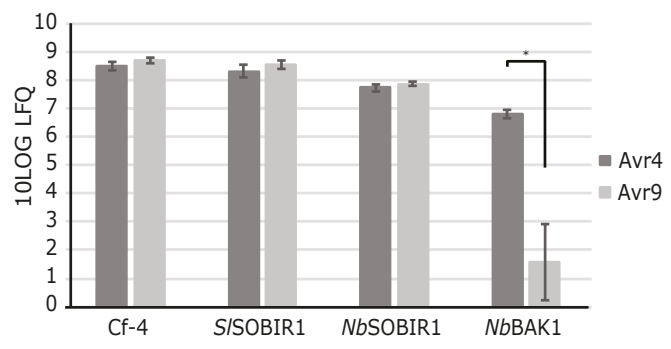

Figure 5. Differential phosphorylation of SOBIR1 downstream of Cf-4 in the absence or presence of the Avr4 effector could not be shown. (A) AtSOBIR1 in complex with Cf-4 is phosphorylated in planta, but the phosphorylation status of Arabidopsis and tomato SOBIR1 in complex with Cf-4 does not visibly change upon elicitation by Avr4, when using the Pro-Q stain. The indicated Myc-tagged SOBIR1 variants were transiently co-expressed with $\mathrm{P} 19$ in stable transgenic N. benthamiana:Cf-4-eGFP plants, both at an $\mathrm{OD}_{600}$ of 1 . About 40 hours after agroinfiltration, before cell death became apparent, leaves were treated with either $5 \mu \mathrm{M}$ Avr4 (4) or water (w), harvested after $25 \mathrm{~min}$, and subjected to IP using GFP-trap beads. The Rubisco background-band indicates equal loading. (B) NbBAK1 is specifically recruited to the activated Cf-4/SOBIR1-complex. SISOBIR1-Myc was transiently expressed in N. benthamiana:Cf-4eGFP plants, followed by infiltration of $5 \mu \mathrm{M}$ Avr4 or Avr9 protein and harvesting 25 min after Avr protein infiltration. Four replicates were used. After IP with GFP-beads, purified proteins were digested with trypsin and peptide samples were analysed by mass spectrometry (MS). Label Free Quantification (LFQ) resulted in the identification of equal amounts of $\mathrm{CF}-4$, SISOBIR1, and endogenous NbSOBIR1. Note that NbBAK1 is significantly enriched in the samples obtained from Avr4-infiltrated leaves (indicated as 'Avr4'), see also Fig. S9. 'Avr9' indicates the peptides obtained from Avr9-infiltrated leaves. Data are represented as mean LFQ of 4 replicates +/- SE. The two asterisks indicate a significant difference $(p<0.001)$, as determined by a student's t-test. Log LFQ values of zero were replaced by a value of 4.7 (a value slightly lower than the lowest measured value), which was done for three of the four BAK1 values in samples treated with Avr9.

Differential phosphorylation of the SOBIR1-KD might specifically take place on only one or several amino acids, and might not have a net effect on the overall phosphorylation status, so such a change would not be visible with a Pro-Q stain (Taylor et al., 2013; Wang et al., 2005). Therefore, we set out to detect differentially phosphorylated peptides of the KD of SOBIR1 using mass spectrometry (MS) of purified Cf-4/SOBIR1 complexes. For this, we overexpressed SISOBIR1-Myc in N. benthamiana:Cf-4-eGFP plants, which is anticipated to stabilize $\mathrm{Cf}-4$, thereby promoting the accumulation of the Cf-4/SOBIR1 complex (Liebrand et al., 2013; Chapter 3). At 25 min after infiltration with either the Avr4 or Avr9 protein, the latter was included as a negative control, Cf- 
4-eGFP was pulled down. The immunoprecipitated protein samples were subjected to tryptic digestion to obtain peptides. MS identified peptides originating from pulleddown Cf-4, transiently expressed SISOBIR1, and endogenous NbSOBIR1. Label free quantification (LFQ) showed that $\mathrm{C} f 4, \mathrm{~S} / \mathrm{SOBIR} 1$, and NbSOBIR1 were present in equal amounts in the samples treated with either Avr4 or Avr9 (Fig. 5B). This observation shows that, in addition to overexpressed SISOBIR1, endogenous NbSOBIR1 is also associated with the $\mathrm{C} f-4$ protein. As expected, we found an enrichment of endogenous NbBAK1 in the samples originating from the leaves treated with Avr4 (Fig. 5B and Fig. S9) (Albert et al., 2015; Postma et al., 2016; Domazakis et al., 2018; Wang et al., 2018). The recruitment of the co-receptor BAK1 confirms that the Cf-4/SOBIR1 complex has been activated by Avr4, and shows that our method to specifically purify the non-activated and activated Cf-4/SOBIR1 complex and its associated signalling partners, is effective. However, no phospho-peptides originating from the KD of SOBIR1 were identified. In a repetition of this experiment we enriched the peptide samples, obtained from the immunoprecipitated protein samples, for phosphorylated peptides with $\mathrm{TiO}_{2}$ beads, before performing the MS analyses. Unfortunately, also in this case no phosphorylated peptides of SOBIR1 were identified. Therefore, possible differential phosphorylation of SOBIR1 in purified protein samples obtained from leaves of Avr4- versus Avr9-treated $N$. benthamiana:Cf-4-eGFP plants, could not be determined. Also a repetition of the experiment employing overexpression of AtSOBIR1-Myc, instead of SISOBIR1-Myc, did not significantly yield any phosphorylated peptides of AtSOBIR1.

Although we cannot draw a conclusion about possible differential phosphorylation of SOBIR1 upon ligand recognition by $\mathrm{Cf}-4$, our results again show that AtSOBIR1 is clearly phosphorylated in planta, as visualized by Pro-Q staining.

\section{The KD of constitutively active AtSOBIR1 is phosphorylated at several positions}

As the co-IP of SOBIR1 via Cf-4-eGFP did not reveal phosphorylated amino acid residues of SOBIR1, we next examined whether the potential phosphorylation sites of SOBIR1 that were found with the mutational analyses, and possibly additional sites, are actually phosphorylated in planta using direct immunoprecipitation of eGFP-tagged SOBIR1. For this we employed overexpression of AtSOBIR1-eGFP, and AtSOBIR1 ${ }^{\mathrm{D} 489 \mathrm{~N}-e G F P}$ as a negative control, in $N$. benthamiana. AtSOBIR1 has been shown before to be phosphorylated in vitro (Leslie et al., 2010) and in planta (Fig. 5A

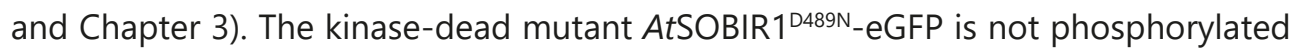
in planta when visualized by a Pro-Q stain (Fig. 5A and Chapter 3). IP of AtSOBIR1-

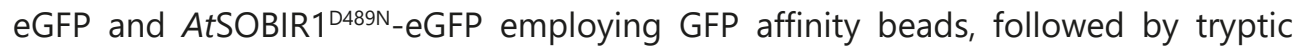
digestion and subsequent enrichment for phosphorylated peptides using $\mathrm{TiO}_{2}$ beads, resulted in the identification of pT519 specifically in wild-type and not in kinase-dead AtSOBIR1 (Table 1). 
Table 1. The KD of AtSOBIR1 is phosphorylated on T519 and several other amino acid residues. Phosphorylated residues found in the kinase domain of AtSOBIR1 in planta, as detected by mass spectrometry of purified phospho-peptides derived from pulled-down eGFP-tagged wild-type (WT) and kinase-dead AtSOBIR1 ${ }^{D 489 N}$. Underlining and a ' $p$ ' in front of a residue indicates that phosphorylation was found on this location, with a localization probability of at least 0.7 in at least 2 out of 4 samples.

\begin{tabular}{|c|c|c|}
\hline \multicolumn{2}{|c|}{ Phosphorylated residue(s) } & \multirow[t]{2}{*}{ Peptide sequence } \\
\hline AtSOBIR1-eGFP & AtSOBIR1 $1^{\mathrm{D} 89 \mathrm{~N}_{-} \mathrm{eGFP}}$ & \\
\hline S326 & - & GSEKPPGPpSIFSPLIK \\
\hline S329 & S329 & GSEKPPGPSIFpSPLIK \\
\hline S369 & - & AELPGpSNGKIIAVK \\
\hline T390 & T390 & VIQPPKDADELPTDEDSKFLNK \\
\hline T390 and S394 & T390 and S394 & DADELpTDEDpSKFLNK \\
\hline S406 & S406 & pSEINTVGHIR \\
\hline T410 & - & SEINpTVGHIR \\
\hline T519, and T522, T523, or S524 & - & AMPDAVpTHIpTpTpSHVAGTVGYIAPEFYQTHK \\
\hline S592 & S592 & NIITSENPpSLAIDPK \\
\hline T634 & - & DVRpTMLSQIK \\
\hline S637 & - & TMLpSQIK \\
\hline
\end{tabular}

The observation of T519 being an in planta phosphorylation site correlates with our mutational analysis, which showed that T519 is essential for immune signalling by SOBIR1 (Fig. 2). Three additional Thr/Ser residues in the activation segment (T522, T523, S524) might also be phosphorylated, however this could not be reliably detected as the localization probability was below the threshold (Table 1). As these potential phosphorylation sites are situated close to each other, they are all located on one peptide after tryptic digestion. Although in the phospho-peptides identified from the activation segment there likely is an additional phosphorylated amino acid present next to pT519, based on the identified MS/MS spectrum we cannot determine whether this is pT522, pT523, or pS524. Because peptides with multiple phosphorylated residues may not elute efficiently from the $\mathrm{TiO}_{2}$ beads (Li et al., 2009), it is possible that peptides with a third, fourth, or even a fifth phosphorylated residue might be missed during our analysis.

Additionally, several other Thr and Ser phosphorylated peptides of AtSOBIR1 were found. As expected, most phospho-peptides were identified in the samples originating from wild-type AtSOBIR1, however, we also identified a few phosphopeptides in samples where kinase-dead AtSOBIR1 $1^{\mathrm{D} 489 \mathrm{~N}}$ was transiently expressed and purified (Table 1). Phosphorylation of these sites is apparently independent of AtSOBIR1-kinase activity. As AtSOBIR1 $1^{\mathrm{D} 489 \mathrm{~N}}$ is not able to signal for immunity, phosphorylation of these sites is likely to not be important for immune signalling (Liebrand et al., 2013; Chapter 3). Possibly, these phospho-peptides are an artefact 
of the heterologous overexpression of kinase-dead AtSOBIR1, which accumulates to relatively high levels in $N$. benthamiana.

Next to phosphorylation of amino acid residues present in the activation segment of AtSOBIR1, phospho-peptides were found that revealed phosphorylation of other Thr residues of wild-type AtSOBIR1, namely pS326, pS369, pT410, pT634, and pT639 (Table 1). S326 is located in the intracellular juxtamembrane (iJM) domain, just adjacent of the KD. On this location a Thr is present in Solanaceous SOBIR1 homologues. Possibly, this site is involved in signalling, as phosphorylation of iJM domains has earlier been shown to play a role in signalling by RLKs (Oh et al., 2009; Xu et al., 2006). S369 is located in the N-lobe, in the region just after the Gly-rich loop and is conserved in several SOBIR1 homologues (Fig. S1B). T410, located in the $\alpha C$ helix of the $\mathrm{N}$-lobe, is not conserved in Solanaceous homologues of SOBIR1 (Fig. S1B). In the 3D model of the AtSOBIR1-KD, T410 appears to be in close proximity to the N-terminal anchor of the activation segment. T634 and S637 are located in the $\alpha \mathrm{K}$ helix, which is the C-terminal end of the KD, and both orient outwards. T634 is not conserved amongst SOBIR1 homologues, but S637 is present in most depicted SOBIR1-homologues (Fig. S1B). Future mutational analysis should shed light on the possible role of these phosphorylation sites in immune signalling by SOBIR1.

We did not identify any Tyr phosphorylation, using this $\mathrm{TiO}_{2}$ purification approach, which might be explained by the low abundance of this modification and the transient nature of the presence of phosphate groups on Tyr residues (Olsen et al., 2006; van der Mijn et al., 2015).

These data show the identification of pT519 as a specific phosphorylation site of the activation loop of AtSOBIR1 in planta. In addition, several additional phosphorylated residues are identified in our studies.

\section{Discussion}

\section{Potential phosphorylation sites in the KD of SOBIR1 are essential for its immune function}

SOBIR1 is a key regulatory RLK that associates with RLPs to form bimolecular RLKs enabling signalling from the cell surface to the cytoplasm (Liebrand et al., 2014; Gust \& Felix, 2014). Phosphorylation is a crucial mechanism that acts as molecular switch in various cellular processes, including plant defence and development. Here, we have identified several potential phosphorylation sites in the KD of SOBIR1 that are crucial for the function of SOBIR1 in immune signalling. We show that T519 and T522, located in the activation loop of SOBIR1, are essential for SOBIR1-functioning, and are likely to be involved in the interaction of the AtSOBIR1-KD with signalling partners. Moreover, MS analysis confirmed the phosphorylation of T519 in planta. 
Additionally, we show that T523 and T529 are also essential for SOBIR1 function and likely stabilize the KD by interacting with the RD motif. Y532 is probably involved in determining the efficiency of binding to substrate proteins. Y538 is important to stabilize the activation loop, and Y436 supports the interaction of K377 and E407 in the $\mathrm{N}$-lobe, and is essential to keep the SOBIR1-KD in its active conformation.

Potential phosphorylation sites in the activation loop of SOBIR1 are essential for its immune function

T519 and T522, both residues that are located in the activation loop of the SOBIR1$K D$ and point outwards to enable interactions with potential signalling partners, are highly conserved in the KD of SOBIR1 homologues (Fig. S1B and 4A). Our mutational analysis shows their importance in signalling for immunity by SOBIR1, using the constitutive immune response as a read-out, as well as restoration of the immune response downstream of Cf-4 in complementation assays (Fig. 2). Interestingly, we detected phospho-peptides with pT519 originating from AtSOBIR1-eGFP pull downs (Table 1). Phosphorylation on T522 is likely, but could not be unambiguously distinguished with the identified phospho-peptides (Table 1). Interestingly, pro-Q staining revealed that $A t S O B I R 1^{T 519 A}$ has a strongly reduced overall phosphorylation level, but AtSOBIR1 ${ }^{\text {T522A }}$ partially retained its overall phosphorylation (Fig. $2 \mathrm{C}$ and S3). This indicates that pT519 is essential to keep the SOBIR1-KD in an active conformation, and that pT522 is likely necessary for specific signalling. The equivalents of the residues T519 and T522 of AtSOBIR1 in AtBAK1 are T446 and T449, and in AtBRI1 they are T1039 and S1042 (note that this is a Ser instead of a Thr). These sites have been shown to be phosphorylated in vitro and in vivo, both for AtBRI1 and AtBAK1 (Wang et al., 2005 and 2008). Analysis of the functionality of mutants of these particular amino acids in planta showed no clear phenotype for AtBAK1 concerning BR and flagellin-related signalling (Wang et al., 2008), but did show a clear role for T1039 and S1042 of AtBRI1 in BR signalling (Wang et al., 2005). Interestingly, phosphorylation of these residues in both AtBAK1 and AtBRI1 affects the orientation of the activation loop to obtain an active conformation of the kinase and to allow interaction with signalling partner proteins (Yan et al., 2012; Bojar et al., 2014). So, together, this indicates that pT519 is essential to keep the SOBIR1-KD in an active conformation. Furthermore, pT519 and also PT522 likely enable interactions with signalling partners. Therefore, T519 is likely to be a primary phosphorylation site, and T522 might be transphosphorylated by signalling partner BAK1 (Chapter 3), leading to differential specificity for potential signalling partners.

S524, also points outwards of the activation loop of the SOBIR1-KD, but is partly covered by the $\mathrm{N}$-lobe and seems to play a less important role in signalling by SOBIR1 than the Thr residues in the activation segment (Fig. S1B, Fig. 2, Fig 4A). Although AtSOBIR1 $1^{\text {S524A }}$ is impaired in MAPK activation, it has an overall phosphorylation 
level comparable to wild-type AtSOBIR1, and is able to, at least partially, activate constitutive and Cf-4-triggered immune responses (Fig. 2). Maybe there is more than one signalling pathway downstream of SOBIR1, one dependent and one independent of the particular MAPKs that we visualised using antibodies against phosphorylated MAPKs (Fig. 2B). The antibody we used, anti-p42/p44-erk, visualizes the activation of MAPK3/6, but there are many more MAPKs that might also transduce a signal downstream of Cf-4 (Lee et al., 2015; Zhang et al., 2018). Also, signalling for immune responses without the need of MAPK activation has recently been discovered, as FLS2 signals via BIK1 directly to RBOHD to produce ROS (Kadota et al., 2014; Li et al., 2014). So, S524 does play a role in MAPK3/6 activation, but is not essential for immune signalling by SOBIR1.

AtSOBIR $1^{\mathrm{T} 523}$ points inwards into the $\mathrm{KD}$, and is in close proximity to the positively charged Arg (R488) residue of the RD motif (Fig. 4B). Phosphorylation of T523 is likely to facilitate the interaction with R488 to position the activation segment and the catalytic loop to obtain the active conformation of the SOBIR1-KD. pT450 of AtBAK1, which corresponds to AtSOBIR $1^{\mathrm{pT} 523}$, is also oriented towards the Arg residue of the RD motif, thereby stabilizing the catalytic loop (Yan et al., 2012). Interestingly, the AtBAK1 ${ }^{\mathrm{T} 450 \mathrm{~A}}$ mutation had only a partial effect on growth and development, but diminished FLS2 signalling (Wang et al., 2008; Yan et al 2012). Our mutational studies show that T523 is essential for immune signalling by SOBIR1, but not required for its overall phosphorylation (Fig. 2). Phosphorylation on T523 is plausible, but could not be distinguished with the identified phospho-peptides (Table 1). Together, this leads to the conclusion that pT523, possibly transphosphorylated by the signalling partner BAK1 (Chapter 3), is essential for the KD of SOBIR1 to specifically signal for the activation of immune responses.

\section{Potential phosphorylation sites in the $P+1$ loop and $\alpha E F / \alpha F$ loop of SOBIR 1 are essential for its immune function}

The catalytic Asp in the RD motif is essential to correctly orient the hydroxyl group of the protein substrate to be phosphorylated towards the ATP gamma phosphate that will be transferred (Kornev et al., 2006; Adams, 2003). AtSOBIR1 ${ }^{\text {T529, }}$, located in the $\mathrm{P}+1$ loop, is oriented towards this Asp (D489) residue, and is likely to be important to assist with its proper orientation (Fig. 4B). The equivalents of AtSOBIR1 ${ }^{\text {T529 }}$ in AtBAK1 and AtBRI 1 are T455 and T1049, respectively. Mutations of these Thr residues to Ala lead to nearly complete loss of kinase activity, and to loss of flagellin-related and BR signalling (Wang et al., 2008; Wang et al., 2005). AtBAK $1^{\mathrm{T} 455}$ and AtBRI1 ${ }^{\mathrm{T} 1049}$ are also oriented in the direction of the catalytic Asp and the substrate, so the phosphorylation of these Thr residues is suggested to play a role in kinase activity and substrate binding (Yan et al., 2012; Bojar et al., 2014). In concert with this, AtSOBIR1 ${ }^{\text {T529A }}$ lost its overall phosphorylation and its ability to signal for immunity, similar to kinase- 
dead AtSOBIR1 $1^{\text {D489N }}$ (Fig. 2). Therefore, T529 is probably important to keep the KD of SOBIR1 in its active conformation, and either its hydroxyl group or phosphorylation of T529 is essential for this.

We observed increased constitutive immune activity of the AtSOBIR $1^{\mathrm{YS} 32 \mathrm{~F}}$ mutant when compared to wild-type AtSOBIR1, as cell death occurred faster and required lower ODs of the Agrobacterium suspension used for the infiltrations (Fig. S5C). Y532 is located in the P+1 loop, which is important for substrate binding (Nolen et al., 2004; Kornev \& Taylor, 2010), and is oriented to the substrate-binding pocket of the SOBIR1-KD (Fig. 4B). Interestingly, Y532 is not completely conserved among the various SOBIR1 homologues of various plant species (Fig. S1B) and different tomato species (Aflitos et al., 2014), so essential phosphorylation of this residue is not likely. Probably, the hydroxyl group of $\mathrm{Y} 532$ has an inhibitory effect on substrate binding.

Adjacent of the activation segment is the $\alpha E F / \alpha F$ loop (Fig. 1 and S1B). The $\alpha E F /$ $\alpha F$ loop is important to stabilize the activation loop, which folds on the $\alpha E F / \alpha F$ loop (Nolen et al., 2004). After phosphorylation of the activation loop, the $\alpha E F / \alpha F$ loop helps to stabilize the phosphorylated activation loop in the active conformation. Furthermore, the $\alpha E F / \alpha F$ loop destabilizes the conformation of a non-active, unphosphorylated activation loop, suppressing refolding into the active state (Nolen et al., 2004). $\mathrm{Y} 538$ is located in the $\alpha \mathrm{EF} / \alpha \mathrm{F}$ loop of AtSOBIR1 (Fig. 4A and S1B). For the corresponding Tyr residue in AtBRI1, Y1057, loss of catalytic activity in vitro was shown for the AtBRI1 ${ }^{\text {Y1057F }}$ mutant (Oh et al., 2009), indicating an essential role of this Tyr residue in the activity of the BRI1-KD. Whether phosphorylation of Y1057 in AtBRI1 is essential, or the hydroxyl group of the Tyr residue itself is required for an active conformation, is not clear. Constitutive immune activity of AtSOBIR $1^{1538 F}$ is strongly compromised (Fig. $3 \mathrm{~A}$ and $\mathrm{B}$ ). However, AtSOBIR1 $1^{\mathrm{Y} 538 \mathrm{~F}}$ shows some residual overall phosphorylation in planta, and still partially restores the Cf-4/Avr4-induced $\mathrm{HR}$ in complementation assays (Fig. $3 \mathrm{C}$ and D). The structural presence of the hydroxyl group of $Y 538$, or its phosphorylation, is likely to contribute to the stabilization of the activation loop. As a consequence, Y538 affects the activity of the SOBIR1-KD and/or the affinity of its interaction with signalling partners that are involved in downstream responses.

\section{A potential phosphorylation site in the C-lobe of the SOBIR1-KD is essential for its activity}

The strongly conserved residue Y436, present at the C-lobe of the KD of SOBIR1, is essential for its constitutive immune activity, for its overall phosphorylation, and for signalling downstream of Cf-4/Avr4 (Fig. 3). Mutational analyses of the corresponding Tyr residue in AtBAK1, in which Y363 was replaced by an Ala or a Glu residue, showed that this conserved Tyr residue is important in maintaining an active conformation of the ATP binding pocket and trans-autophosphorylation activity of the AtBAK1-KD 
(Yan et al., 2012). Additionally, the corresponding residue in AtBRI1, Y956, has been shown to be a trans-autophosphorylation site, independent of BR perception, and appeared to be essential for AtBRI1 kinase activity in vitro and in vivo (Oh et al., 2009). In the 3D model of the KD of AtSOBIR1, Y436 is in close proximity to the Lys/Glu pair of the N-lobe (Fig. 4C). The Lys/Glu pair is in general important for the stabilization of the active conformation of a KD (Kornev et al., 2006). Likely, the hydroxyl group of Y436, or its phosphorylation, is important to assist with the stabilization of an active conformation of the SOBIR1-KD.

As Y438 is located in the hinge region between the $\mathrm{N}$ - and the C-lobe (S1B and Fig. S8), the mild suppression of the constitutive immune response and MAPK activation in AtSOBIR $1^{\mathrm{Y} 438 \mathrm{~F}}$ mutants might be caused by a mild effect on the conformation of the KD (Fig. 3). The mutational analyses presented here, suggest that the residues Y433, Y476, Y550, and Y617 do not play an important role in the function of SOBIR1 in immunity (Fig. 3). For Y617 this was expected, as for this residue only a low sequence conservation was found amongst the SOBIR1-KDs of various plants (Fig. S1). Y433, Y476, and Y550 are to some extent conserved (Fig. S1), but apparently their hydroxyl group and/or their possible phosphorylation does not play an essential role in SOBIR1-mediated defence signalling. The homologous residue of AtSOBIR1 $1^{\mathrm{Y} 46}$ in AtEFR, Y836, is phosphorylated upon EFR activation by the elf18 peptide, and is essential for the function of EFR in immunity (Macho et al., 2014). As EFR is a nonRD kinase, whereas SOBIR1 is an RD kinase, a difference in the mode of action of both KDs is likely (Johnson et al., 1996; Nolen et al., 2004; Kornev et al., 2006). Y428 in Chitin Elicitor Receptor Kinase 1 (AtCERK1), the residue that is also homologous to AtSOBIR $1^{\mathrm{Y} 476}$, has recently been shown to be a trans-autophosphorylation site, essential for chitin-triggered defence responses (Liu et al., 2018). CERK1 contains a LysM extracellular domain, and although this RLK has an RD kinase domain, the overall homology with SOBIR1 is low, so different functioning is likely.

SOBIR1 is also known as Evershed (EVR) (Leslie et al., 2010), and EVR was discovered in a mutant screen in Arabidopsis, where a knockout of EVR restored flower organ abscission in a nevershed (nev) mutant background. It would be interesting to test whether our AtSOBIR1 mutants would still be able to complement the nev evr double mutant phenotype, as this could help to determine specific phosphorylation sites that allow the SOBIR1-KD to differentially signal for immunity or to signal for inhibition of organ abscission. 


\section{In planta analyses to reveal the 'real-life' situation}

In the mutational studies, we utilised phospho-dead substitutions of Thr, Ser, and Tyr residues in the KD of SOBIR1 (either by a change to Ala or to Phe), to characterize whether phosphorylation of these residues plays a role in immune signalling by SOBIR1. A phospho-dead mutation resulting in a phenotype, which in our case is scored as impaired constitutive and Cf-4-mediated immune activity, indicates that phosphorylation of the particular residue might be important. In addition, the intrinsic difference between the structure of Thr/Ser and Ala, and between Tyr and Phe, namely the presence of an hydroxyl group or not, could play a role. Phosphoproteomics confirmed that T519, and possibly another Thr or Ser residue, in the activation segment is phosphorylated (Table 1). Additionally, Pro-Q analysis showed that the mutants AtSOBIR1 $1^{1522 \mathrm{~A}}$ and AtSOBIR1 $1^{\mathrm{T} 23 \mathrm{~A}}$ are still phosphorylated upon their overexpression in planta, pointing to the conclusion that their change from Thr to Ala does not affect the intrinsic activity of the KD of SOBIR1, so likely the phosphorylation of these residues itself plays a role, for example in their specific interaction with downstream signalling partners (Fig. 2C). For the other sites we cannot strictly conclude whether the hydroxyl group itself or altered phosphorylation of the amino acid plays a functional role in signalling by SOBIR1.

As additional proof for the observed importance of the potential phosphorylation sites, it would be interesting to introduce phospho-mimic mutations by substituting these residues by a negatively charged residue Asp or Glu residue (Wang et al., 2008; Oh et al., 2012). Mutation of the important Thr and Ser residues to either Asp or Glu is anticipated to lead to a highly active KD of AtSOBIR1, associated with elevated constitutive immune responses, if phosphorylation of the Thr or Ser residue is important for immune activity. However, Asp or Glu substitutions of Tyr residues are rarely successful, as Asp and Glu have a structure very different from Tyr. We have generated Asp mutants for Y436, Y532, and Y538 of AtSOBIR1, but they did not accumulate properly, or resulted in a phospho-dead phenotype.

\section{A model explaining early phosphorylation events of the Cf-4/SOBIR1 complex upon activation by Avr4}

Our mutational analyses revealed the importance of several Thr residues in the activation segment, and of three tyrosine residues, of which one is located in the $\mathrm{N}$-lobe, one in the $\mathrm{P}+1$ loop, and one in the $\alpha \mathrm{EF} / \alpha \mathrm{F}$ loop. The characterisation of phospho-peptides of transient in planta-expressed AtSOBIR1-eGFP confirmed the phosphorylation of T519 and one other Thr/Ser in the activation loop (Table 1). As SOBIR1 is an RD kinase, we hypothesise that T519, T523, and T529 are so-called primary-phosphorylation sites. Trans-autophosphorylation on these residues to enable signalling upon Avr4 recognition by $\mathrm{Cf}-4$, is likely to be necessary to activate the KD of SOBIR1, and lock it in an active conformation (Yan et al., 2012). After the 
recruitment of BAK1 and its phosphorylation by SOBIR1, trans-phosphorylation by activated BAK1 on T522, Y532, and Y538 might facilitate differential activity of SOBIR1, in view of substrate specificity and affinity to signalling partners (Fig. 6).

Whether the phosphorylation sites of SOBIR1 are trans-autophosphorylated by SOBIR1 itself, or transphosphorylated by its recruited signalling partner BAK1 (Chapter 3), or another potential signalling partner like an RLCK, cannot actually be concluded from the current data that we have available. However, it is likely that some residues, mainly the hypothesised primary phosphorylation sites, are trans-autophosphorylation sites, as SOBIR1 has been shown to form homodimers in planta (Chapter 3). Transphosphorylation by a signalling partner, e.g. BAK 1 , has been proposed before (Chapter 3), and is likely to occur upon ligand recognition by the RLP and subsequent BAK1 recruitment to the RLP/SOBIR1 complex (Albert et al., 2015; Postma et al., 2016). Possibly, differential phosphorylation after the initiation of downstream signalling also occurs to negatively regulate SOBIR1-KD activity. For example, phosphorylation on Y532 might negatively regulate the activity of the SOBIR1-KD, as unphosphorylated Y532 has been shown to be very active in immune signalling (Fig. 3). Additionally, transphosphorylation by an RLCK seems obvious, as has been shown for FLS2-BAK1 signalling with BIK1 (Lu et al., 2010), but remains to be studied.

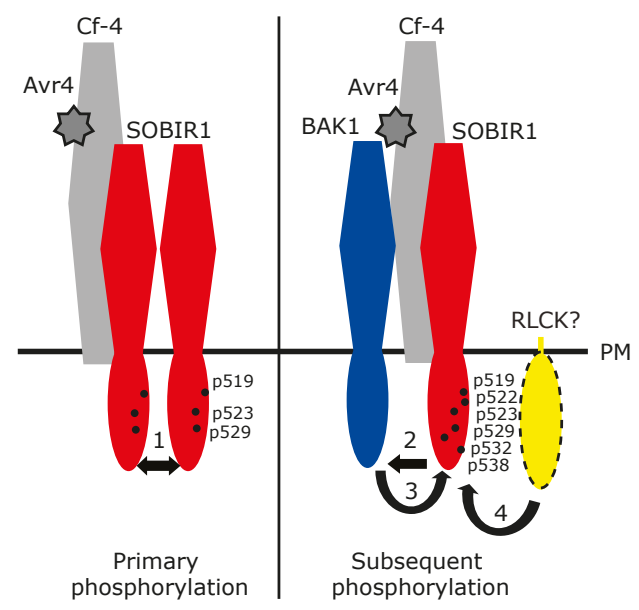

Figure 6. A model explaining early phosphorylation events of the Cf-4/SOBIR1/BAK1 complex upon its activation by Avr4. In this model we propose that Cf-4-associated homodimers of SOBIR1 trans-autophosphorylate on T5219, T523, and T529 to obtain an active conformation (left), upon Avr4 elicitation of Cf-4. Subsequently, BAK1 is recruited (right), as a result of the altered phosphorylation status of the SOBIR1-KD and/or as a result of the increased affinity of BAK1 to Avr4-activated Cf-4 compared to Cf-4 in the resting state. Likely, transphosphorylation between BAK1 and SOBIR1 takes place, and BAK1mediated phosphorylation of SOBIR1 on T522 and Y538 fully activates the tri-partite complex to enable downstream signalling. Possibly, an RLCK is also involved and might positively regulate SOBIR1 activity via transphosphorylation events. Furthermore, negative regulation might take place via phosphorylation of Y532. pY532 is anticipated to block substrate binding to SOBIR1 and thereby negatively regulate immune signalling. 
TofurthervalidatewhichSOBIR1-phosphorylationsitesaretrans-autophosphorylated or transphosphorylated by signalling partners like BAK1, in vitro production of the SOBIR1-KD and its here presented mutants, in combination with the KD of BAK1, and subsequent kinase activity assays and phosphoproteomics (Taylor et al., 2013) might shed light on the order by which phosphorylation of SOBIR1 and BAK1 occurs, and which sites might be trans-autophosphorylated and which transphosphorylated.

\section{Future outlook}

Unfortunately, we could not identify sites of the KD of SOBIR1 that are differentially phosphorylated downstream of $\mathrm{Cf}-4$, when the $\mathrm{Cf}-4 / \mathrm{SOBIR} 1$ complex is activated by Avr4. Despite several attempts, employing transient expression of AtSOBIR1 and SISOBIR1 in N. benthamiana:Cf-4-eGFP, either with or without Avr4 treatment, we could not detect phospho-peptides by MS. We hypothesised that transient expression of SOBIR1 would increase the amounts of endogenous Cf-4-eGFP due to stabilisation of the RLP (Liebrand et al., 2013; Chapter 3). Subsequent pull-down of Cf-4-eGFP after treatment with either Avr4 or water, would then allow the isolation of the differentially activated pool of Cf-4-associated SOBIR1. However, phosphopeptides derived from the KD of SOBIR1 were not identified, neither when using SISOBIR1 nor AtSOBIR1. Possibly, the amount of phosphorylated SOBIR1 protein obtained in this way was too low to identify phospho-peptides, as a direct pull-down of AtSOBIR1-eGFP did result in the identification of such phospho-peptides (Table 1). Nevertheless, our IP experiment pulling down Cf-4-eGFP without the enrichment for phospho-peptides, revealed co-immunoprecipitation of SOBIR1, and recruitment of endogenous NbBAK1 to the activated Cf-4/SOBIR1 complex upon treatment with Avr4, which means that our method is appropriate. It is likely that activated RLP/SOBIR1complexes are strictly down regulated in activity, for example via dephosphorylation, and/or via endocytosis followed by ubiquitination and degradation (Couto \& Zipfel., 2016), which could explain why we cannot find phosphorylated SOBIR1 peptides after Avr4-elicitation. Additionally, the enrichment of phospho-peptides might not be sensitive enough. To increase the chance of success, future research might benefit from the use of other techniques to enrich for phospho-peptides, for instance IMAC for multi-phosphorylated T/S peptides, and affinity-purification with beads for $\mathrm{pY}$ peptides (Macek et al., 2007; Bodenmiller et al., 2009; Mithoe \& Menke, 2015; van der Mijn et al., 2015). Also simultaneous measuring of specifically synthesised phosphopeptides could help to reveal the presence of the predicted phospho-peptide (Macho et al., 2014).

The phosphorylation sites in the KD of SOBIR1 that were identified upon in plantaexpression of AtSOBIR1-eGFP were partly unexpected (Table 1). As SOBIR1 proteins with a phosphorylation pattern that supports the activation of downstream signalling probably have a quick turnover, as a result of endocytosis and subsequent degradation, 
they likely do not really accumulate. Therefore, these signalling-competent versions of SOBIR1 are probably extremely difficult to isolate. As a consequence, the pattern of phosphorylation that we found for AtSOBIR1 might not represent the signallingcompetent pattern of the SOBIR1-KD, but possibly reflects residual phosphorylation of partially activated AtSOBIR1 proteins.

The current mutational study was performed using AtSOBIR1, because it allowed us to use the constitutive immune activity of AtSOBIR1 in tobacco and $N$. benthamiana, as well as its ability to complement for signalling downstream of $\mathrm{Cf}-4$, as read-outs for its functionality and thereby determine the effect of the various mutations on this functionality. Because there is a high sequence homology between AtSOBIR1 and SISOBIR1, and most of the targeted Thr, Ser, and Tyr residues are highly conserved, we are confident that the phosphorylatable residues that we found to be important in the KD of AtSOBIR1 are also essential in SISOBIR1. Nevertheless, future studies would benefit from including SISOBIR1 in experiments. To allow complementation assays in $N$. benthamiana involving mutants of SISOBIR1, we could make use of a synthetic sequence of SISOBIR1, with an altered codon use, in such a way that VIGS of endogenous NbSOBIR1, which has a high sequence homology with SISOBIR1, will not target the transiently expressed SISOBIR1 mutant sequence. Additionally, the novel genome editing technique CRISPR-Cas, should enable the fast generation of SOBIR1-knockouts in tomato and $N$. benthamiana. Complementation studies in such knockout plants, instead of in silenced plants, has the additional advantage that there is no endogenous SOBIR1 present, in contrast to NbSOBIR1(/-like)-silenced plants in which NbSOBIR1 gene expression is only knocked-down.

\section{Materials \& Methods}

\section{Binary vectors for Agrobacterium-mediated transformation and virus-induced gene silencing (VIGS)}

The constructs pGWB20-35S::SICf-4-Myc, pBIN-KS-35S::AtSOBIR1-eGFP, pBIN-KS-

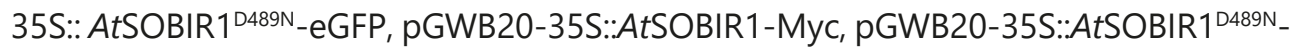

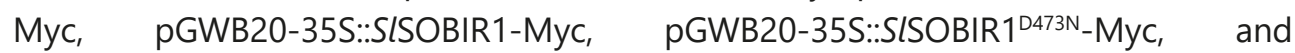
PTRV2:NbSOBIR1/NbSOBIR1-like have been described previously (Liebrand et al., 2012 and 2013). C. fulvum effectors Avr4 and Avr9 were expressed using pMOG800 constructs (van der Hoorn et al., 2000). P19 (Voinnet et al., 2015) and pTRV1 (Liu et al., 2002a and 2002b) have been described elsewhere.

To generate AtSOBIR1 mutant constructs with single Tyr residues changed into a Phe residue that cannot be phosphorylated, or Thr/Ser residues changed into Ala residues, also resulting in removal of a phosphorylation site, polymerase chain reactions (PCRs) were performed with pENTR/D-Topo-AtSOBIR1(no-stop) (Sol 
2843, Liebrand et al., 2013) as a template, using the QuickChange Site-Directed Mutagenesis Kit (Stratagene). Primer sets covering the target region were designed to introduce a single nucleotide mutation, thereby generating a Tyr to Phe or a Thr/Ser to Ala codon change (Table S1). Mutant clones were confirmed through sequencing, and introduced into pBIN-KS-35S::GWY-eGFP (Sol 2095, for C-terminal tagging with eGFP) by LR reaction with Gateway LR Clonase enzyme mix II (Invitrogen) to obtain binary vectors for in planta expression. The resulting binary vectors $\mathrm{PBIN}$ -

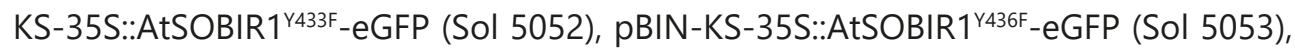

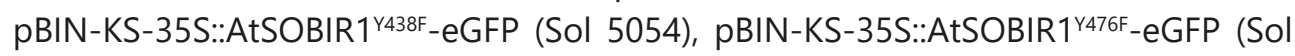

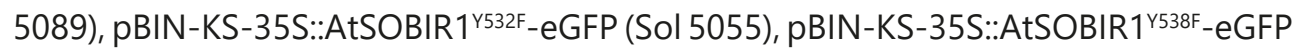
(Sol 5063), pBIN-KS-35S::AtSOBIR1 $1^{\text {Y550F }}$-eGFP (Sol 5056), pBIN-KS-35S:::AtSOBIR1 $1^{\text {Y617F- }}$ eGFP (Sol 5093), pBIN-KS-35S::AtSOBIR1 $1^{\text {T519A }}$-eGFP (Sol 5137), pBIN-KS35S::AtSOBIR1 1522A_eGFP (Sol 5138), pBIN-KS-35S::AtSOBIR1 1523A-eGFP (Sol 5139), pBIN-KS-35S::AtSOBIR1 1524A-eGFP (Sol 5140), and pBIN-KS-35S::AtSOBIR1 1529A-eGFP (Sol 5141) were transformed to Agrobacterium tumefaciens strain $\mathrm{C} 58 \mathrm{C} 1$, carrying the pCH32 helper plasmid. pBIN-KS-35S::GUS-eGFP (SOL 5094) was constructed by a PCR (see Table S1 for primer sequences) on pENTR-GUS (Invitrogen), cloning into pENTR/D-Topo, and a subsequent LR reaction with Gateway LR Clonase enzyme mix II (Invitrogen) to obtain the binary vector.

\section{Plant growth conditions}

Nicotiana tabacum (tobacco, CV. SR1) and N. benthamiana (wild-type, N. benthamiana stably expressing SICf-4 under its native promoter (referred to as $N$. benthamiana:Cf-4) (Gabriëls et al., 2006), and N. benthamiana stably expressing SICf4-eGFP under $35 \mathrm{~S}$ promoter (referred to as N. benthamiana:Cf-4-eGFP (Postma et al., 2016)), were grown under $16 \mathrm{~h}$ of light at $25^{\circ} \mathrm{C}$ and $8 \mathrm{~h}$ darkness at $21^{\circ} \mathrm{C}$, at $\sim 75 \%$ relative humidity.

\section{Phylogenetic analyses of the SOBIR1 kinase domain}

Homologous protein sequences of AtSOBIR1 were retrieved from phytozome (phytozome.jgi.doe.gov/pz/portal.html) and the Sol Genomics Network (www. solgenomics.net), and only the kinase domains were aligned using the Mafft webserver (Stamatakis et al., 2008). An unrooted maximum likelihood tree was built using phylogeny.fr (Dereeper et al., 2008 and 2010), using G-blocks to eliminate poorly aligned positions and divergent regions, and viewed using MEGA.

Variations in sequence within Arabidopsis ecotypes (Weigel \& Mott, 2009) and tomato species (Aflitos et al., 2014), were analysed using Ensemble plant (plants. ensembl.org/index.html). 


\section{Three dimensional modelling of the SOBIR1 kinase domain}

A 3D model of the KD of AtSOBIR1 was built using the online expasy modelling tool (swissmodel.expasy.org) (Biasini et al., 2014; Benkert et al., 2010), and is based on the crystal structure the KD of ATP-bound BRI1 (4q5j.2.A), which was the best fitting template (GMQE 0.68, QMEAN -2.27, sequence identity 40.88, resolution $2.77 \AA$, sequence similarity 0.40 , coverage 0.93 ).

\section{Agrobacterium-mediated transient transformation}

Agrobacterium-mediated transient transformations (agroinfiltrations) were performed as previously described (van der Hoorn et al., 2000). Agroinfiltrations to monitor constitutive immune activity by AtSOBIR1 variants in tobacco (SR1) and $N$. benthamiana, which can be observed as cell death, was performed at an $\mathrm{OD}_{600}$ of 1 , unless stated otherwise. Where indicated, co-infiltration of the silencing suppressor P19 in $N$. benthamiana was performed at an $\mathrm{OD}_{600}$ of 1 . A four-way score for visual cell death was used: $100 \%$ for complete cell death, $60 \%$ for intermediate cell death, $30 \%$ for weak cell death, and $0 \%$ for no cell death. The significance of observed differences in cell death scores was tested with one-way ANOVA, including a Tukey post hoc-test (Bewick et al., 2004).

To monitor changes in the phosphorylation status of the KD of SOBIR1 upon elicitor recognition by $\mathrm{Cf}-4$, we infiltrated $5 \mu \mathrm{M}$ Avr4 or Avr9 protein, two days after agroinfiltration of Myc-tagged SOBIR1 variants together with P19, and harvested leaf material after 25 minutes.

\section{Protein immunoprecipitation and identification}

Immunoprecipitations (IPs) and co-IPs were performed as described previously (Liebrand et al., 2013). To detect phosphorylated proteins, a protein extraction buffer (pEB) was used as described by Karlova and co-workers (2006 and 2009), with minor modifications (Chapter 3). After one hour incubation with the leaf extract, the beads were washed five times with pEB for co-IP experiments, and for all other experiments three times washing was performed. Pre-cast TGX gels were used for Pro-Q and Sypro Ruby staining (Bio-Rad \#456-1095) and TGX stain-free gels were employed for all other analyses (Bio-Rad \#456-8085). Total protein loaded on gel was visualized using the stain-free method or with Coomassie Brilliant Blue (CBB) staining. Pro-Q diamond phosphoprotein gel stains and subsequent Sypro Ruby stains were done according to the manufacturer's recommendations (Invitrogen) (Taylor et al., 2013). The following antibodies were used for protein detection on immuno blots (IB):

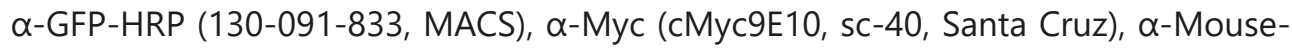
HRP (GE healthcare), anti-p42/p44-erk (NEB) and goat anti-rabbit (Sigma). Band intensities were quantified using Image Lab software, and ratios were calculated as 
indicated in the figure legends. Significance of the difference in intensities was tested with one-way ANOVA, including a Tukey post hoc-test (Bewick et al., 2004).

IPs with the aim to analyse the purifying proteins via mass spectrometry (MS), were performed on at least $5 \mathrm{gram}$ of $N$. benthamiana leaf material. The pEB buffer was used for protein extraction, and $10 \mathrm{ml}$ of protein extract was subjected to IP with $50 \mu$ l of the GFP-affinity beads suspension (Chromotek). After the IP, the pulled-down proteins were subjected to on-bead digestion (Wendrick et al., 2017), with the minor modification that after a 2 hours incubation with trypsin (Promega), the beads were removed and the supernatant was incubated overnight with another $0.5 \mu \mathrm{l}$ trypsin. Hereafter, the peptides were cleaned-up (Wendrich et al., 2017), or first enriched for phospho-peptides using the $\mathrm{TiO}_{2}$ method described by Aryal and co-workers (2010) without the addition of $\mathrm{KCl}$, and then cleaned-up (Wendrich et al., 2017).

The peptide samples were measured by Proxeon LTQ-Orbitrap XL nLC-MS/MS as described (Wendrich et al., 2017). LC-MS runs with all MS/MS spectra obtained were analysed with MaxQuant 1.5.2.8 (Cox \& Mann, 2008), using the 'Specific Trypsin/ $\mathrm{P}^{\prime}$ digestion mode, with maximally 2 missed cleavages and further default settings for the Andromeda search engine (first search $20 \mathrm{ppm}$ peptide tolerance, main search $4.5 \mathrm{ppm}$ tolerance, IT-MS/MS fragment match tolerance of 0.5 Da, Carbamidomethyl (C) set as a fixed modification, while variable modifications were set for Protein $\mathrm{N}$-terminal Acetylation and $\mathrm{M}$ oxidation which were completed by non-default settings for de-amidation of $\mathrm{N}$ and $\mathrm{Q}$ and phosphorylation of $\mathrm{S}, \mathrm{T}$ and/or $\mathrm{Y}$, the maximum number of modifications per peptide was 5 (Cox et al., 2011)).

$N$. benthamiana database downloaded from Uniprot (www.uniprot.org), supplemented with additional protein sequences from the infiltrated proteins, was used together with a contaminants database that contains sequences of common contaminants, like Trypsins (P00760, bovin and P00761, porcin) and human keratins (Keratin K22E (P35908), Keratin K1C9 (P35527), Keratin K2C1 (P04264) and Keratin $\mathrm{K} 1 \mathrm{Cl}$ (P35527)). The 'label-free quantification' (LFQ), as well as the 'match between runs' options were enabled. De-amidated peptides were allowed to be used for protein quantification and all other quantification settings were kept default.

Filtering and further bioinformatic analysis of the MaxQuant/Andromeda workflow output, and the analysis of the abundance of the identified proteins, were performed with the Perseus 1.5.5.3 module (available at the MaxQuant suite). The false discovery rate (FDR) was set to $1 \%$ and proteins with at least 2 identified peptides, of which at least one should be unique and at least one should be unmodified, were accepted.

Reversed hits were deleted from the MaxQuant result table as well as all results showing a normalised LFQ intensity value of 0 for both sample and control. The $\log 10$ logarithm was taken from protein LFQ MS1 intensities as obtained from MaxQuant. Zero 'Log LFQ' values were replaced by a value of 4.7 (a value slightly lower than the lowest measured value) to make ratio calculations possible. Relative 
protein quantitation of sample to control was done with Perseus by applying a two sample T-test using the LFQ intensity obtained with FDR set to 0.05 and S0 set to 1 .

For the $\mathrm{TiO}_{2}$ enriched phospho-peptides, peptides were considered phosphorylated when a localization probability of at least 0.7 in at least 2 out of 4 samples was found in the MaxQuant Phospho(STY) sites table.

\section{Heterologous complementation of SOBIR1 in $\mathbf{N}$. benthamiana}

VIGS using TRV-based vectors was performed in N. benthamiana:Cf-4 as described previously (Liebrand et al., 2012). For the heterologous complementation assays of SOBIR1 (Liebrand et al., 2013), transient co-transformation of the generated AtSOBIR1eGFP phospho-site mutants with Avr4 or Avr9 of C. fulvum in N. benthamiana:Cf-4 was performed at an $\mathrm{OD}_{600}$ of 0.7 , with Avr4 or Avr9 at an $\mathrm{OD}_{600}$ of $0.03,3$ weeks after agro-inoculation with recombinant pTRV2:NbSOBIR1/-like to silence endogenous NbSOBIR1. The resulting cell death (hypersensitive response, HR) was scored at 3 days post infiltration (dpi) in a randomized, blind way. The four-way score for the cell death was used. The significance of the cell death score was tested with a one-way ANOVA, including a Duncan's post hoc-test (Bewick et al., 2004).

\section{Protein localization studies}

Protein localization studies were performed as described previously (Chapter 3).

\section{Acknowledgements}

We thank Unifarm personnel for excellent plant care. We acknowledge Laurens Deurhoffor help with cloning, Cris Wijnen for help with statistics, and Thomas Liebrand for help with analysing the MS data. A.M.v.d.B. is supported by the Netherlands Organization for Scientific Research (NWO), Earth and Life Sciences (ALW). Research by J.P. and S.R. is supported by the Gatsby Charitable Foundation. 


\section{References}

Adams JA. 2003. Activation loop phosphorylation and catalysis in protein kinases: is there functional evidence for the autoinhibitor model? Biochemistry 42: 601-607.

Adams JA. 2001. Kinetic and catalytic mechanisms of protein kinases. Chemical Reviews 101: 22712290.

Aflitos S, Schijlen E, Jong H, Ridder D, Smit S, Finkers R, Wang J, Zhang G, Li N, Mao L. 2014. Exploring genetic variation in the tomato (Solanum section Lycopersicon) clade by wholegenome sequencing. The Plant Journal 80: 136-148.

Afzal AJ, Wood AJ, Lightfoot DA. 2008. Plant receptor-like serine threonine kinases: roles in signaling and plant defense. Molecular Plant-Microbe Interactions 21: 507-517.

Albert I, Böhm H, Albert M, Feiler CE, Imkampe J, Wallmeroth N, Brancato C, Raaymakers TM, Oome S, Zhang H, Krol E, Grefen C, Gust AA, Chai J, Hedrich R, Van den Ackerveken G, Nürnberger T. 2015. An RLP23-SOBIR1-BAK1 complex mediates NLP-triggered immunity. Nature Plants 1: 15140.

Aryal UK, Ross AR. 2010. Enrichment and analysis of phosphopeptides under different experimental conditions using titanium dioxide affinity chromatography and mass spectrometry. Rapid Communications in Mass Spectrometry 24: 219-231.

Benkert P, Biasini M, Schwede T. 2010. Toward the estimation of the absolute quality of individual protein structure models. Bioinformatics 27: 343-350.

Bewick V, Cheek L, Ball J. 2004. Statistics review 9: One-way analysis of variance. Critical Care 8: 130-136.

Biasini M, Bienert S, Waterhouse A, Arnold K, Studer G, Schmidt T, Kiefer F, Cassarino TG, Bertoni M, Bordoli L. 2014. SWISS-MODEL: modelling protein tertiary and quaternary structure using evolutionary information. Nucleic Acids Research 42: W252-W258.

Bodenmiller B, Mueller LN, Mueller M, Domon B, Aebersold R. 2007. Reproducible isolation of distinct, overlapping segments of the phosphoproteome. Nature Methods 4: 231.

Böhm H, Albert I, Fan L, Reinhard A, Nürnberger T. 2014. Immune receptor complexes at the plant cell surface. Current Opinion in Plant Biology 20: 47-54.

Bojar D, Martinez J, Santiago J, Rybin V, Bayliss R, Hothorn M. 2014. Crystal structures of the phosphorylated BRI1 kinase domain and implications for brassinosteroid signal initiation. The Plant Journal 78: 31-43.

Chinchilla D, Zipfel C, Robatzek S, Kemmerling B, Nürnberger T, Jones JD, Felix G, Boller T. 2007. A flagellin-induced complex of the receptor FLS2 and BAK1 initiates plant defence. Nature 448: 497-500.

Couto D, Zipfel C. 2016. Regulation of pattern recognition receptor signalling in plants. Nature Reviews Immunology 16: 537-552.

Cox J, Mann M. 2008. MaxQuant enables high peptide identification rates, individualized p.p.b.range mass accuracies and proteome-wide protein quantification. Nature Biotechnology 26: 1367.

Cox J, Neuhauser N, Michalski A, Scheltema RA, Olsen JV, Mann M. 2011. Andromeda: a peptide search engine integrated into the MaxQuant environment. Journal of Proteome Research 10: 1794-1805.

De Wit PJ. 1977. A light and scanning-electron microscopic study of infection of tomato plants by virulent and avirulent races of Cladosporium fulvum. European Journal of Plant Pathology 83: 109-122.

Dereeper A, Blanc G, Claverie J-M, Audic S. 2010. BLAST-EXPLORER helps you building datasets for phylogenetic analysis. BMC Evolutionary Biology 10: 8. 
Dereeper A, Guignon V, Blanc G, Audic S, Buffet S, Chevenet F, Dufayard J-F, Guindon S, Lefort V, Lescot M. 2008. Phylogeny. fr: robust phylogenetic analysis for the non-specialist. Nucleic Acids Research 36: W465-W469.

Domazakis E, Wouters D, Visser R, Kamoun S, Joosten MHAJ, Vleeshouwers VGAA. 2018. The ELR-SOBIR1 complex functions as a two-component RLK to mount defense against Phytophthora infestans. Molecular Plant-Microbe Interactions. 10.1094/mpmi-09-17-0217-r

Gabriëls SHEJ, Takken FLW, Vossen JH, De Jong CF, Liu Q, Turk SCHJ, Wachowski LK, Peters J, Witsenboer HMA, De Wit PJGM, Joosten MHAJ. 2006. cDNA-AFLP combined with functional analysis reveals novel genes involved in the hypersensitive response. Molecular Plant-Microbe Interactions 19: 567-576.

Gao M, Wang X, Wang D, Xu F, Ding X, Zhang Z, Bi D, Cheng YT, Chen S, Li X, Zhang Y. 2009. Regulation of cell death and innate immunity by two receptor-like kinases in Arabidopsis. Cell Host and Microbe 6: 34-44.

Gust AA, Felix G. 2014. Receptor like proteins associate with SOBIR1-type of adaptors to form bimolecular receptor kinases. Current Opinion in Plant Biology 21: 104-111.

Heese A, Hann DR, Gimenez-Ibanez S, Jones AME, He K, Li J, Schroeder JI, Peck SC, Rathjen JP. 2007. The receptor-like kinase SERK3/BAK1 is a central regulator of innate immunity in plants. Proceedings of the National Academy of Sciences of the United States of America 104: 1221712222.

Hohmann U, Lau K, Hothorn M. 2017. The structural basis of ligand perception and signal activation by receptor kinases. Annual Review of Plant Biology 68: 109-137.

Hohmann U, Santiago J, Nicolet J, Olsson V, Spiga FM, Hothorn LA, Butenko MA, Hothorn M. 2018. Mechanistic basis for the activation of plant membrane receptor kinases by SERK-family coreceptors. Proceedings of the National Academy of Sciences of the United States of America 115: 3488-3493.

Johnson LN, Lewis RJ. 2001. Structural basis for control by phosphorylation. Chemical Reviews 101: 2209-2242.

Johnson LN, Noble MEM, Owen DJ. 1996. Active and inactive protein kinases: Structural basis for regulation. Cell 85: 149-158.

Kadota Y, Sklenar J, Derbyshire P, Stransfeld L, Asai S, Ntoukakis V, Jones Jonathan D, Shirasu K, Menke F, Jones A, Zipfel C. 2014. Direct regulation of the NADPH Oxidase RBOHD by the PRR-associated kinase BIK1 during plant immunity. Molecular Cell 54: 43-55.

Karlova R, Boeren S, Russinova E, Aker J, Vervoort J, de Vries S. 2006. The Arabidopsis somatic embryogenesis receptor-like kinase 1 protein complex includes brassinosteroid-insensitive1. The Plant Cell 18: 626-638.

Karlova R, Boeren S, van Dongen W, Kwaaitaal M, Aker J, Vervoort J, de Vries S. 2009. Identification of in vitro phosphorylation sites in the Arabidopsis thaliana somatic embryogenesis receptorlike kinases. Proteomics 9: 368-379.

Kornev AP, Haste NM, Taylor SS, Ten Eyck LF. 2006. Surface comparison of active and inactive protein kinases identifies a conserved activation mechanism. Proceedings of the National Academy of Sciences of the United States of America 103: 17783-17788.

Kornev AP, Taylor SS. 2010. Defining the conserved internal architecture of a protein kinase. Biochimica et Biophysica Acta (BBA)-Proteins and Proteomics 1804: 440-444.

Lee J, Eschen-Lippold L, Lassowskat I, Böttcher C, Scheel D. 2015. Cellular reprogramming through mitogen-activated protein kinases. Frontiers in Plant Science 6: 940.

Leslie ME, Lewis MW, Youn JY, Daniels MJ, Liljegren SJ. 2010. The EVERSHED receptor-like kinase modulates floral organ shedding in Arabidopsis. Development 137: 467-476.

Li J, Wen J, Lease KA, Doke JT, Tax FE, Walker JC. 2002. BAK1, an Arabidopsis LRR receptor-like protein kinase, interacts with BRI1 and modulates brassinosteroid signaling. Cell 110: 213-222. 
Li L, Li M, Yu L, Zhou Z, Liang X, Liu Z, Cai G, Gao L, Zhang X, Wang Y, Chen S, Zhou J-M. 2014. The FLS2-associated kinase BIK1 directly phosphorylates the NADPH oxidase RbohD to control plant immunity. Cell Host \& Microbe 15: 329-338.

Li Q-r, Ning Z-b, Tang J-s, Nie S, Zeng R. 2009. Effect of peptide-to-TiO2 beads ratio on phosphopeptide enrichment selectivity. Journal of Proteome Research 8: 5375-5381.

Liebrand TWH, Smit P, Abd-El-Haliem A, de Jonge R, Cordewener JHG, America AHP, Sklenar J, Jones AME, Robatzek S, Thomma BPHJ, Tameling WIL, Joosten MHAJ. 2012. Endoplasmic reticulum-quality control chaperones facilitate the biogenesis of $\mathrm{Cf}$ receptor-like proteins involved in pathogen resistance of tomato. Plant Physiology 159: 1819-1833.

Liebrand TWH, van den Berg GCM, Zhang Z, Smit P, Cordewener JHG, America AHP, Sklenar J, Jones AME, Tameling WIL, Robatzek S, Thomma BPHJ, Joosten MHAJ. 2013. Receptorlike kinase SOBIR1/EVR interacts with receptor-like proteins in plant immunity against fungal infection. Proceedings of the National Academy of Sciences of the United States of America 110: 10010-10015.

Liebrand TWH, van den Burg HA, Joosten MHAJ. 2014. Two for all: receptor-associated kinases SOBIR1 and BAK1. Trends in Plant Science 19: 123-132.

Lin W, Li B, Lu D, Chen S, Zhu N, He P, Shan L. 2014. Tyrosine phosphorylation of protein kinase complex BAK1/BIK1 mediates Arabidopsis innate immunity. Proceedings of the National Academy of Sciences of the United States of America 111: 3632-3637.

Lin W, Lu D, Gao X, Jiang S, Ma X, Wang Z, Mengiste T, He P, Shan L. 2013. Inverse modulation of plant immune and brassinosteroid signaling pathways by the receptor-like cytoplasmic kinase BIK1. Proceedings of the National Academy of Sciences of the United States of America 110: 12114-12119.

Liu J, Liu B, Chen S, Gong B-Q, Chen L, Zhou Q, Xiong F, Wang M, Feng D, Li J-F, Wang H-B, Wang J. 2018. A tyrosine phosphorylation cycle regulates fungal activation of a plant receptor Ser/Thr kinase. Cell Host \& Microbe 23: 241-253.

Liu Y, Schiff M, Dinesh-Kumar S. 2002a. Virus-induced gene silencing in tomato. The Plant Journal 31: 777-786.

Liu Y, Schiff M, Marathe R, Dinesh-Kumar S. 2002b. Tobacco Rar1, EDS1 and NPR1/NIM1 like genes are required for $\mathrm{N}$-mediated resistance to tobacco mosaic virus. The Plant Journal 30: 415-429.

Lu D, Wu S, Gao X, Zhang Y, Shan L, He P. 2010. A receptor-like cytoplasmic kinase, BIK1, associates with a flagellin receptor complex to initiate plant innate immunity. Proceedings of the National Academy of Sciences of the United States of America 107: 496-501.

Macek B, Mann M, Olsen JV. 2009. Global and site-specific quantitative phosphoproteomics: principles and applications. Annual Review of Pharmacology and Toxicology 49: 199-221.

Macho AP, Lozano-Durán R, Zipfel C. 2015. Importance of tyrosine phosphorylation in receptor kinase complexes. Trends in Plant Science 20: 269-272.

Macho AP, Schwessinger B, Ntoukakis V, Brutus A, Segonzac C, Roy S, Kadota Y, Oh MH, Sklenar J, Derbyshire P, Lozano-Durán R, Gro Malinovsky F, Monaghan J, Menke FL, Huber SC, Yang He S, Zipfel C. 2014. A bacterial tyrosine phosphatase inhibits plant pattern recognition receptor activation. Science 343: 1509-1512.

Macho AP, Zipfel C. 2014. Plant PRRs and the activation of innate immune signaling. Molecular Cell 54: 263-272.

Mithoe SC, Menke FL. 2015. Phosphopeptide Immuno-Affinity Enrichment to Enhance Detection of Tyrosine Phosphorylation in Plants. Plant Phosphoproteomics: Methods and Protocols 1306: 135-146.

Nam KH, Li J. 2002. BRI1/BAK1, a receptor kinase pair mediating brassinosteroid signaling. Cell 110: 203-212.

Nolen B, Taylor S, Ghosh G. 2004. Regulation of protein kinases: Controlling activity through activation segment conformation. Molecular Cell 15: 661-675. 
Oh M-H, Wang X, Clouse SD, Huber SC. 2012. Deactivation of the Arabidopsis BRASSINOSTEROID INSENSITIVE 1 (BRI1) receptor kinase by autophosphorylation within the glycine-rich loop. Proceedings of the National Academy of Sciences of the United States of America 109: 327-332.

Oh M-H, Wang X, Kota U, Goshe MB, Clouse SD, Huber SC. 2009. Tyrosine phosphorylation of the BRI1 receptor kinase emerges as a component of brassinosteroid signaling in Arabidopsis. Proceedings of the National Academy of Sciences of the United States of America 106: 658-663.

Oh MH, Wu X, Clouse SD, Huber SC. 2011. Functional importance of BAK1 tyrosine phosphorylation in vivo. Plant Signaling \& Behavior 6: 400-405.

Olsen JV, Blagoev B, Gnad F, Macek B, Kumar C, Mortensen P, Mann M. 2006. Global, in vivo, and site-specific phosphorylation dynamics in dignaling networks. Cell 127: 635-648.

Postma J, Liebrand TWH, Bi G, Evrard A, Bye RR, Mbengue M, Kuhn H, Joosten MHAJ, Robatzek SC. 2016. Avr4 promotes Cf-4 receptor-like protein association with the BAK1/SERK3 receptorlike kinase to initiate receptor endocytosis and plant immunity. New Phytologist 210: 627-642.

Schwessinger B, Roux M, Kadota Y, Ntoukakis V, Sklenar J, Jones A, Zipfel C. 2011. Phosphorylation-dependent differential regulation of plant growth, cell death, and innate immunity by the regulatory receptor-like kinase BAK1. PLoS Genetics 7: e1002046-e1002046.

Stamatakis A, Hoover P, Rougemont J. 2008. A rapid bootstrap algorithm for the RAxML web servers. Systematic Biology 57: 758-771.

Taylor I, Seitz K, Bennewitz S, Walker JC. 2013. A simple in vitro method to measure autophosphorylation of protein kinases. Plant Methods 9: 22.

van der Hoorn RAL, Laurent F, Roth R, de Wit PJGM. 2000. Agroinfiltration is a versatile tool that facilitates comparative analyses of Avr9/Cf-9-induced and Avr4/Cf-4-induced necrosis. Molecular Plant-Microbe Interactions 13: 439-446.

van der Mijn JC, Labots M, Piersma SR, Pham TV, Knol JC, Broxterman HJ, Verheul HM, Jiménez CR. 2015. Evaluation of different phospho-tyrosine antibodies for label-free phosphoproteomics. Journal of Proteomics 127: 259-263.

Voinnet O, Rivas S, Mestre P, Baulcombe DC. 2015. Retraction: An enhanced transient expression system in plants based on suppression of gene silencing by the p19 protein of tomato bushy stunt virus (retraction of The Plant Journal, vol 33, pg 949, 2003).

Wang X, Goshe MB, Soderblom EJ, Phinney BS, Kuchar JA, Li J, Asami T, Yoshida S, Huber SC, Clouse SD. 2005. Identification and functional analysis of in vivo phosphorylation sites of the Arabidopsis BRASSINOSTEROID-INSENSITIVE1 receptor kinase. The Plant Cell 17: 1685-1703.

Wang X, Kota U, He K, Blackburn K, Li J, Goshe MB, Huber SC, Clouse SD. 2008. Sequential transphosphorylation of the BRI1/BAK1 receptor kinase complex impacts early events in brassinosteroid signaling. Developmental Cell 15: 220-235.

Wang Y, Xu Y, Sun Y, Wang H, Qi J, Wan B, Ye W, Lin Y, Shao Y, Dong S, Tyler BM, Wang Y. 2018. Leucine-rich repeat receptor-like gene screen reveals that Nicotiana RXEG1 regulates glycoside hydrolase 12 MAMP detection. Nature Communications 9: 594.

Weigel D, Mott R. 2009. The 1001 genomes project for Arabidopsis thaliana. Genome Biology 10: 107.

Wendrich JR, Boeren S, Möller BK, Weijers D, De Rybel B. 2017. In vivo identification of plant protein complexes using IP-MS/MS. Plant Hormones. Methods in Molecular Biology 1497: 147158.

Wu J, van der Burgh A, Bi G, Zhang L, Alfano JR, Martin G, Joosten MHAJ. 2018. The bacterial effector AvrPto targets the regulatory co-receptor SOBIR1 and suppresses defence signalling mediated by the receptor-like protein Cf-4. Molecular Plant-Microbe Interactions 31: 75-85.

Xu J, Wei X, Yan L, Liu D, Ma Y, Guo Y, Peng C, Zhou H, Yang C, Lou Z, Shui W. 2013. Identification and functional analysis of phosphorylation residues of the Arabidopsis BOTRYTIS-INDUCED KINASE1. Protein \& Cell 4: 771-781. 
Xu WH, Wang YS, Liu GZ, Chen X, Tinjuangjun P, Pi LY, Song WY. 2006. The autophosphorylated Ser686, Thr688, and Ser689 residues in the intracellular juxtamembrane domain of XA21 are implicated in stability control of rice receptor-like kinase. The Plant Journal 45: 740-751.

Yan L, Ma Y, Liu D, Wei X, Sun Y, Chen X, Zhao H, Zhou J, Wang Z, Shui W, Lou Z. 2012. Structural basis for the impact of phosphorylation on the activation of plant receptor-like kinase BAK1. Cell Research 22: 1304-1308.

Zhang J, Li W, Xiang T, Liu Z, Laluk K, Ding X, Zou Y, Gao M, Zhang X, Chen S, Mengiste T, Zhang Y, Zhou JM. 2010. Receptor-like cytoplasmic kinases integrate signaling from multiple plant immune receptors and are targeted by a Pseudomonas syringae effector. Cell Host \& Microbe 7: 290-301.

Zhang M, Su J, Zhang Y, Xu J, Zhang S. 2018. Conveying endogenous and exogenous signals: MAPK cascades in plant growth and defense. Current Opinion in Plant Biology 45, Part A: 1-10.

Zipfel C. 2014. Plant pattern-recognition receptors. Trends in Immunology 35: 345-351. 


\section{Supplemental data}

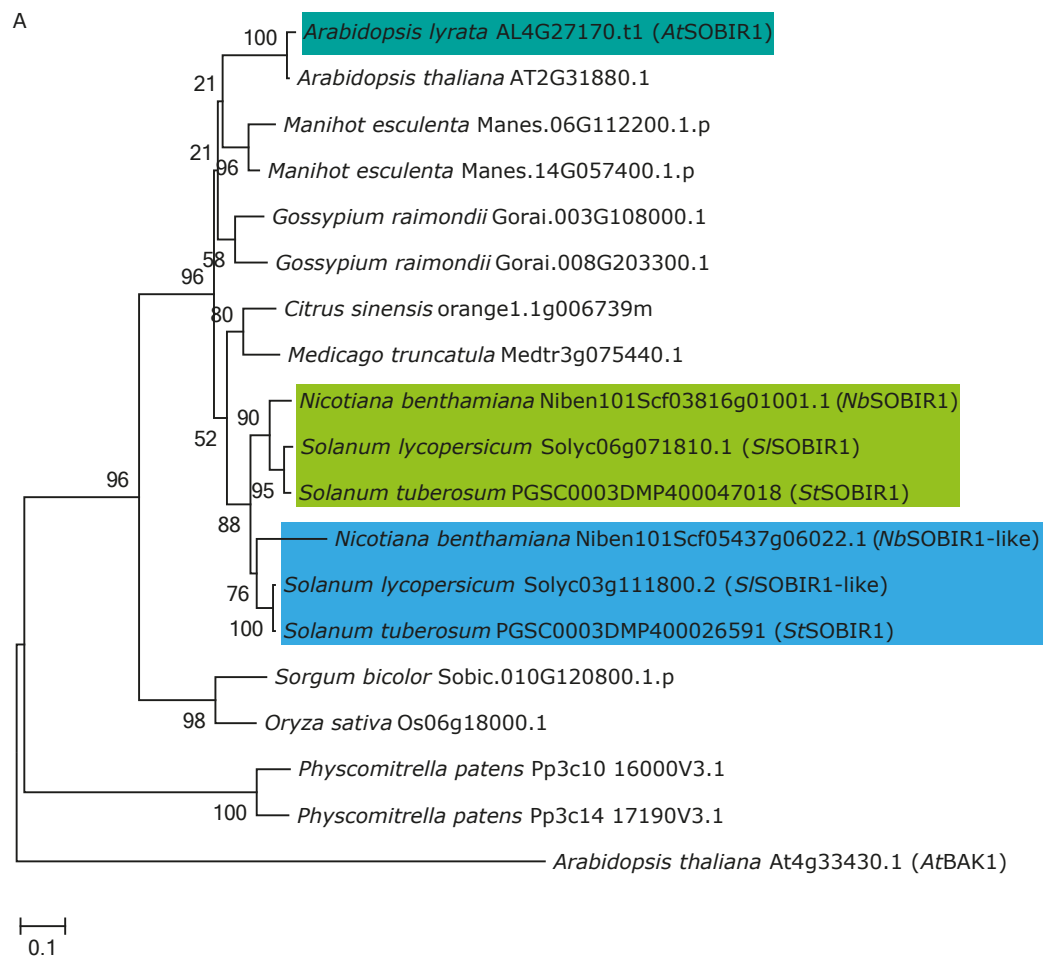

Figure S1. Alignment of the KDs of various SOBIR1 homologues. (A) Phylogenetic tree of SOBIR1KD protein sequences of 12 different plant species. The species were selected to represent a diverse set of well-annotated genomes in the plant kingdom. Bootstrap values are indicated. The branch length indicates genetic change. AtBAK1 is used as an out-group. 


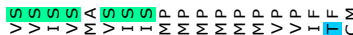

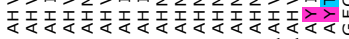
\lrcorner$\lrcorner\lrcorner\lrcorner\lrcorner\lrcorner\lrcorner\lrcorner\lrcorner\lrcorner\lrcorner\lrcorner\lrcorner\lrcorner 匹 \backsim\lrcorner\lrcorner \propto$

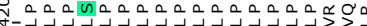

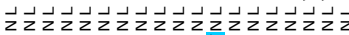

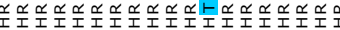

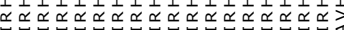

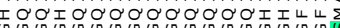

O >>>>>>> $\rightarrow>>>>>>>\lrcorner\lrcorner$

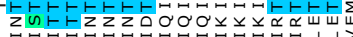

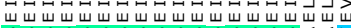

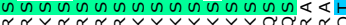

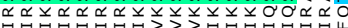

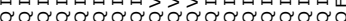

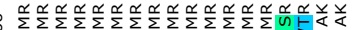

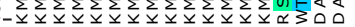

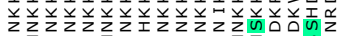

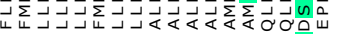

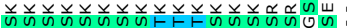

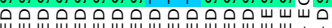

шшшшшшшшшшшшшшш

i

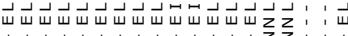
1 1 1 1 1 1 1 $1 z_{2} z$

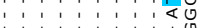

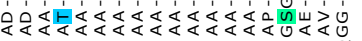

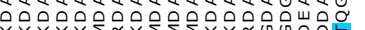

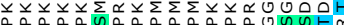

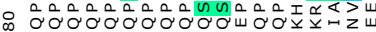

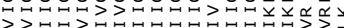

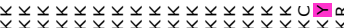

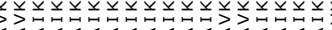

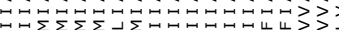

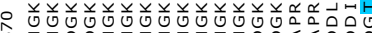

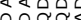

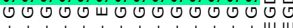

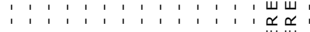

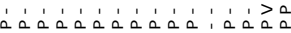

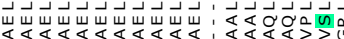

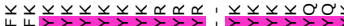

웅

崖出出出出出出出:

U U U U U U U U U U U U U U U U U U U U U : U U U U U U U

,

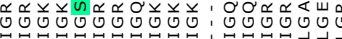

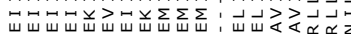

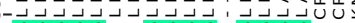

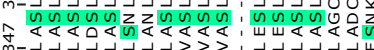

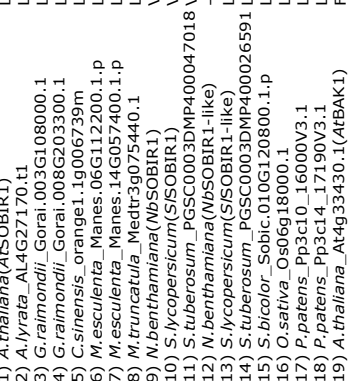

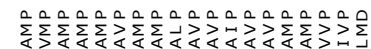

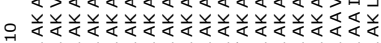
o

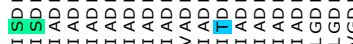

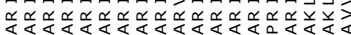

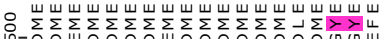

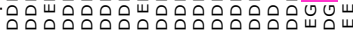

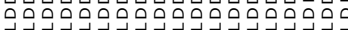

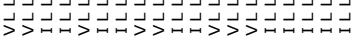

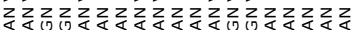

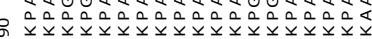

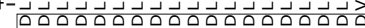

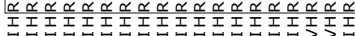

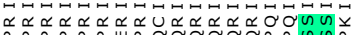
$\begin{array}{ll} & \\ z & 0\end{array}$

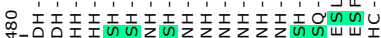

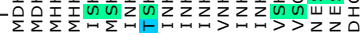
IIIIIIIIIIIIIIIIIII

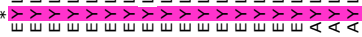

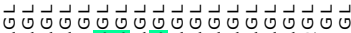

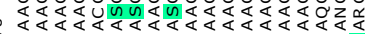
出

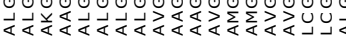

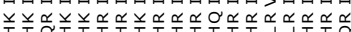

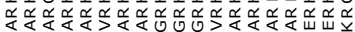

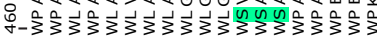

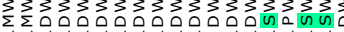

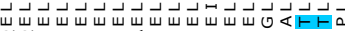

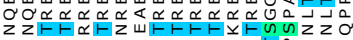
$z_{1} z_{1} F \frac{\alpha}{1}$

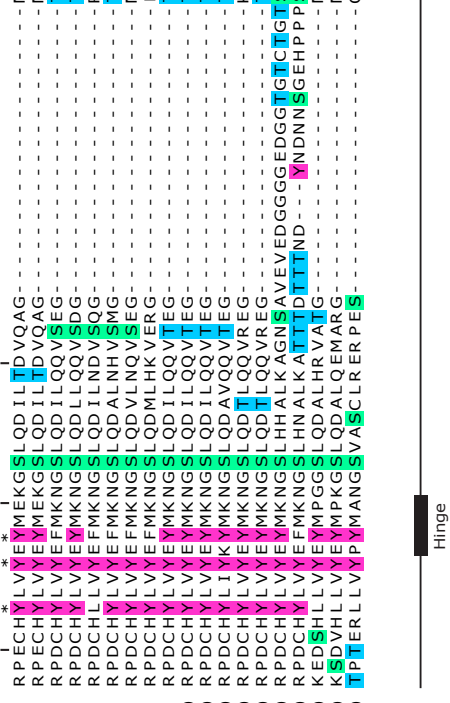

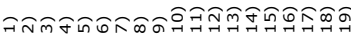




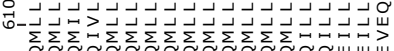
Ơ⿴囗十丁

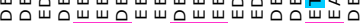

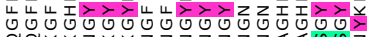

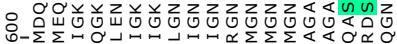

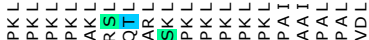

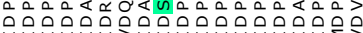

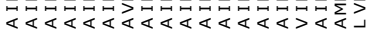

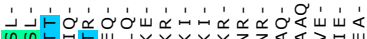

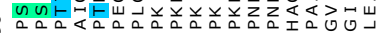

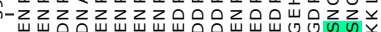

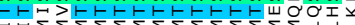

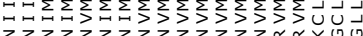
人)

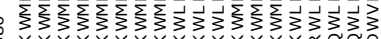
周-

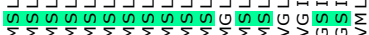

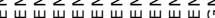

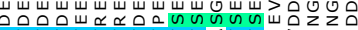

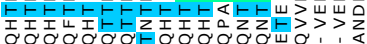
요

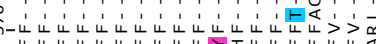

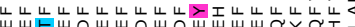

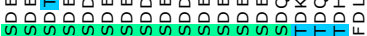

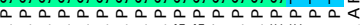

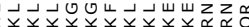

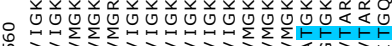

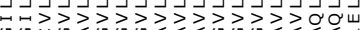

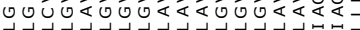

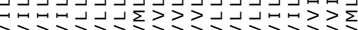
$>>0>0000000000000000$

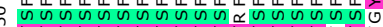

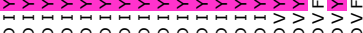

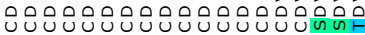

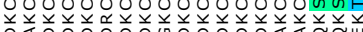

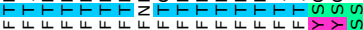

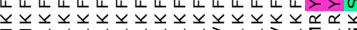

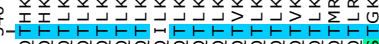

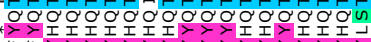

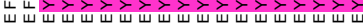

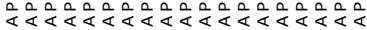

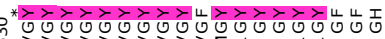

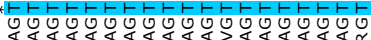
>>

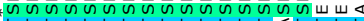

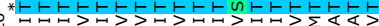

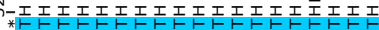
$>>0$

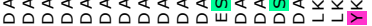

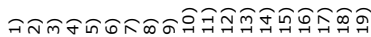
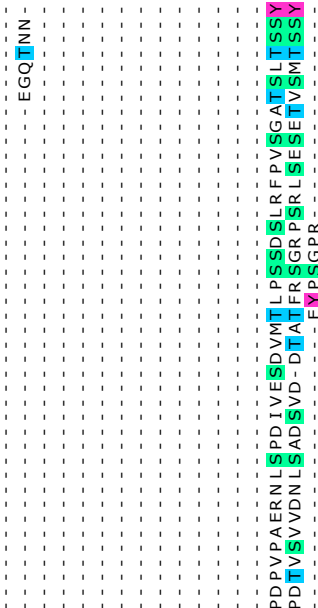

요돈

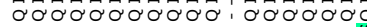
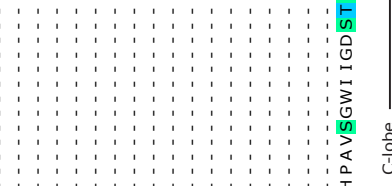

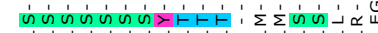

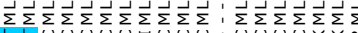

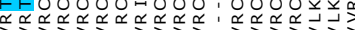
을

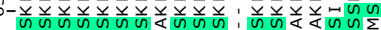

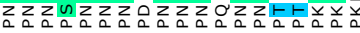

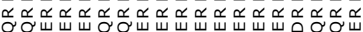

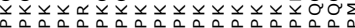

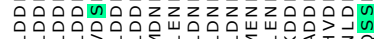

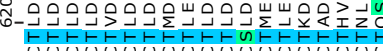

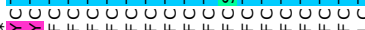

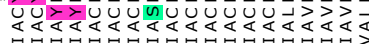

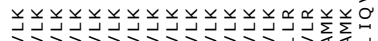

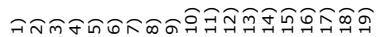

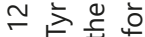

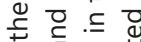

范

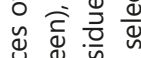

บัญ ญ ญ

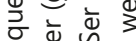

凶थ

.

突 를 을

흘

要

$\frac{1}{2} \rightleftarrows 4$

응 호응 응

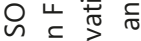

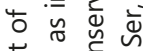

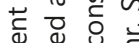

है

은 ․ㅡㄴ

奥 8 这

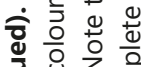

.

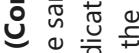

(1)

ปั

施

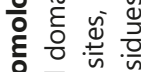

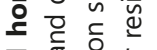

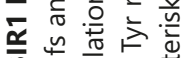

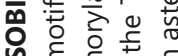

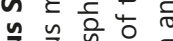

을 을 응 응 专

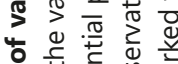

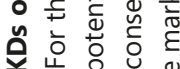

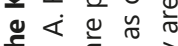

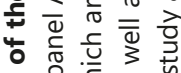

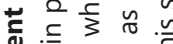

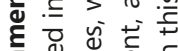

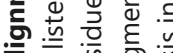

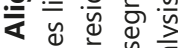

的枈

인

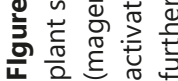


A

tobacco

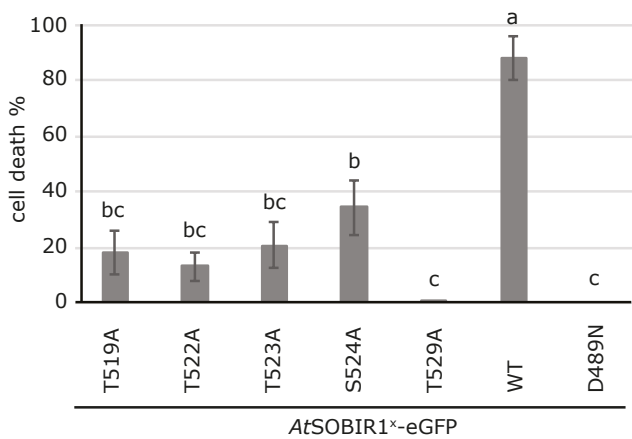

B

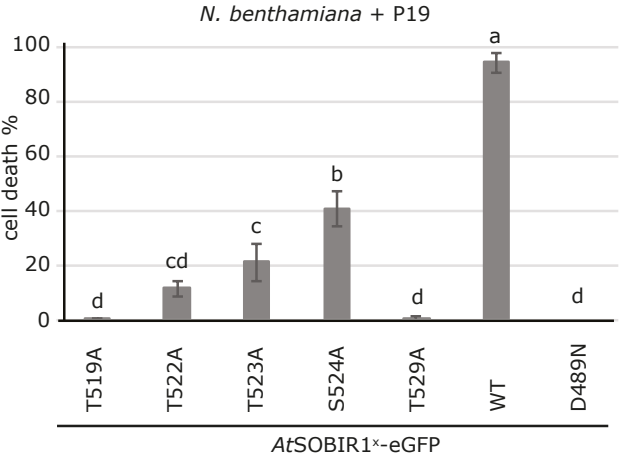

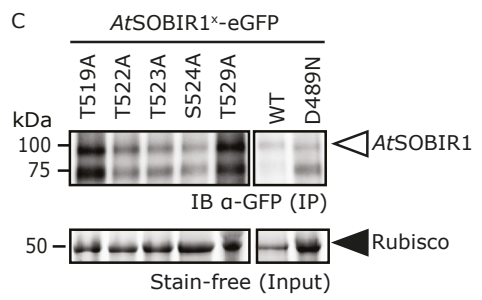

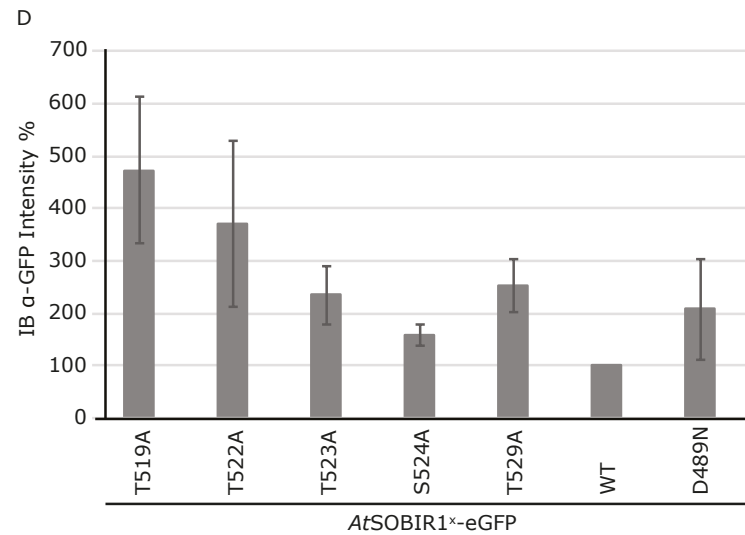

Figure S2. Additional analyses of the AtSOBIR1 Thr and Ser mutants. (A, B) Quantification of cell death triggered by overexpression of the various AtSOBIR1 Thr and Ser mutants, as depicted in Fig. $2 \mathrm{~A}$, in tobacco (A) and $N$. benthamiana (B). Cell death was scored and quantified as described in the Materials \& Methods section. Data are represented as mean +/- standard error (SE). Letters indicate significant differences at $p<0.05$, as determined by one-way ANOVA, including a Tukey post hoc-test. (C) Accumulation levels of the various AtSOBIR1 Thr and Ser mutants negatively correlate with their ability to induce constitutive immunity. The four Thr mutants and one Ser mutant, wild-type AtSOBIR1 (WT), and the kinase-dead mutant AtSOBIR1 ${ }^{1489 N}$, all fused to eGFP, were transiently expressed in $N$. benthamiana, at an $\mathrm{OD}_{600}$ of 1 . To avoid the occurrence of cell death, leaves were harvested already at $1 \mathrm{dpi}$, and after immunoprecipitation (IP) using GFP-affinity beads, the eGFP-tagged proteins were detected by IB (upper panel). The Rubisco band in the total protein reflects the amount of total protein that was employed for the IP (input, lower panel). The experiment was repeated three times, and representative results are shown. (D) Quantification of eGFP-tagged AtSOBIR1 mutant protein accumulation, as determined relative to the Rubisco band intensity of the input, as shown in panel C. Wild-type AtSOBIR1 (WT) accumulation was set to $100 \%$, and percentages are presented as mean +/- SE of three independent experiments. There was no significant difference at $p<0.05$, as determined by one-way ANOVA including a Tukey post hoc-test. 


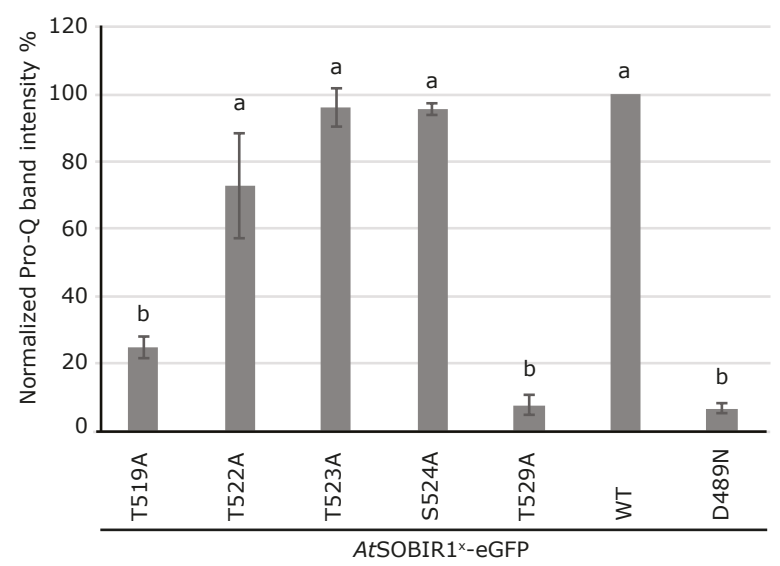

Figure S3. Quantification of the overall phosphorylation levels of the AtSOBIR1 Thr and Ser mutants. Quantification of Pro-Q phosphoprotein staining of the various AtSOBIR1 Thr and Ser mutant proteins, including wild-type (WT) and kinase-dead (D489N) as controls, as shown in Fig. 2C. Note that AtSOBIR1 $1^{\text {T519A }}$, AtSOBIR1 $1^{\text {T529A }}$, and AtSOBIR1 ${ }^{D 489 \mathrm{~N}}$ are significantly reduced in their overall phosphorylation

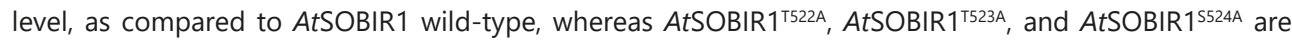
not. Ratios were obtained by dividing the band intensity of pAtSOBIR1 from the Pro-Q staining, by the band intensity reflecting the total amount of pulled-down AtSOBIR1 in the Sypro Ruby-staining, shown in Fig 2 C. The ratio for wild-type AtSOBIR1 was set to $100 \%$ and percentages are presented as mean +/- SE. Letters indicate significant differences at $p<0.05$, determined by one-way ANOVA, including a Tukey post hoc-test. 

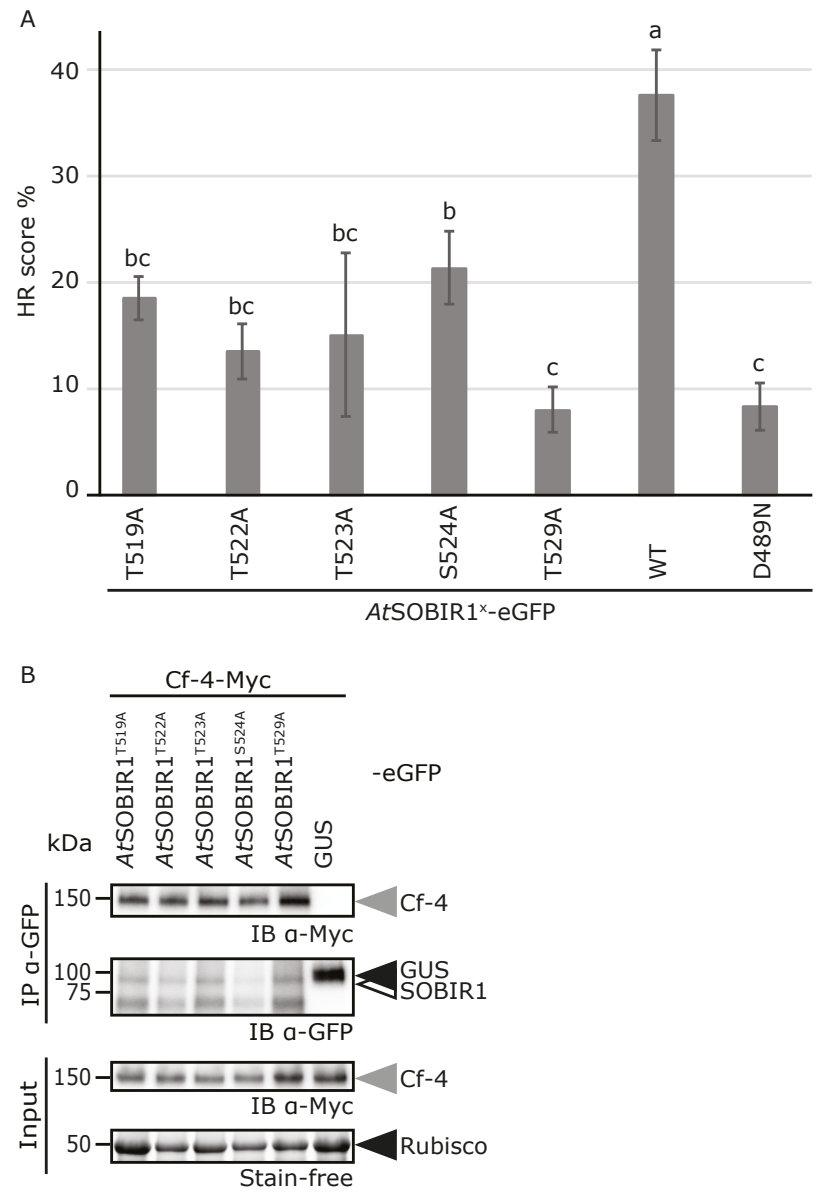

Figure S4. All Thr and Ser residues present in the activation segment of the KD of AtSOBIR1 are required for full SOBIR1 function downstream of Cf-4. (A) Quantification of the intensity of the HR, as shown in Fig. 2D. The HR was scored in a randomized, blind way, and was quantified as described in the Materials \& Methods section. Data are represented as mean $+/-\mathrm{SE}$, letters indicate significant differences at $\mathrm{p}<0.05$, as determined by one-way ANOVA, including a Duncan's post hoc-test. (B) AtSOBIR1 $1^{\mathrm{T} 19 \mathrm{~A}}$, AtSOBIR1 $1^{\text {TS22A }}$, AtSOBIR1 $1^{\text {T523A }}$, AtSOBIR $1^{\text {S524A }}$, and AtSOBIR1 $1^{\text {T529A }}$ all interact with Cf-4. eGFP-tagged versions of the five AtSOBIR1 mutants and Cf-4-Myc were transiently co-expressed in N. benthamiana at an $\mathrm{OD}_{600}$ of 1 , at 1 dpi followed by IP and protein detection by IB. GUS-eGFP was included as a negative control. 
A

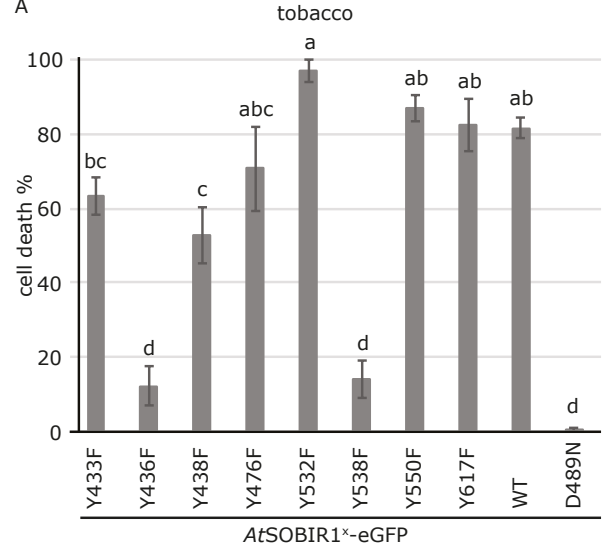

$B$

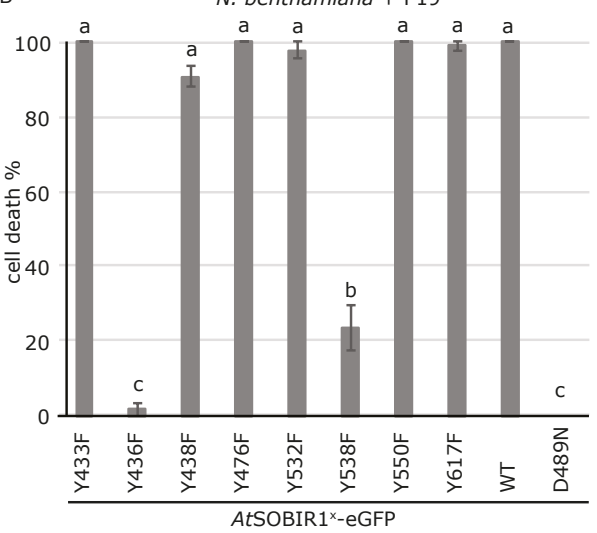

C

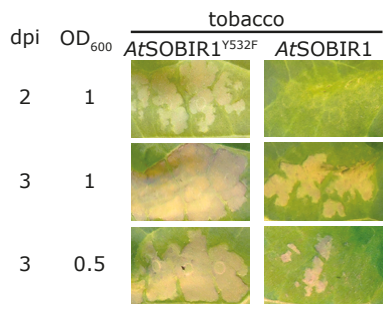

D

AtSOBIR1'-eGFP

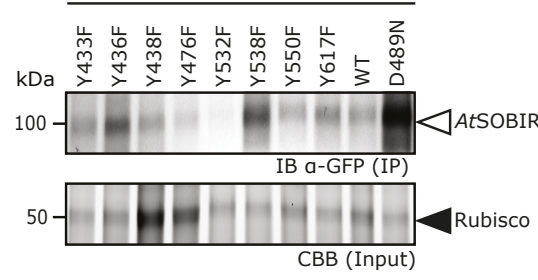

E

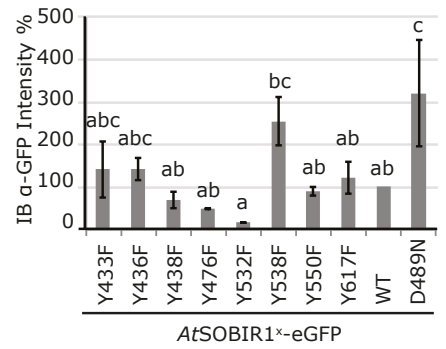

Figure S5. Additional analyses of the various AtSOBIR1 Tyr mutants. (A, B) Quantification of cell death in tobacco $(A)$ and $N$. benthamiana (B), as depicted in Fig. 3A. Cell death was visually scored and quantified as described in the Materials \& Methods section. Data are represented as mean +/- SE. Letters indicate significant differences at $p<0.05$, as determined by one-way ANOVA, including a Tukey post hoc-test. (C) AtSOBIR $1^{\mathrm{YS} 32 \mathrm{~F}}$ shows enhanced constitutive immune activity. Agroinfiltration of AtSOBIR1 $1^{\mathrm{Y} 332 \mathrm{~F}}$ results in the induction of cell death one day earlier, when compared to wild-type AtSOBIR1 (upper four panels). At $3 \mathrm{dpi}$, clear cell death induction by AtSOBIR $1^{\mathrm{Y} 532 \mathrm{~F}}$, as compared to only a weak induction of cell death by wild-type AtSOBIR1, is observed when using a lower $\mathrm{OD}_{600}$ of 0.5 of the $A$. tumefaciens suspension for agroinfiltration (lower two panels). (D) Accumulation levels of the AtSOBIR1 Tyr mutants negatively correlate with their ability to induce constitutive immunity. The eight Tyr mutants, AtSOBIR1 (WT), and the kinase-dead mutant AtSOBIR $1^{D 489 \mathrm{~N}}$ all fused to eGFP, were transiently expressed in $N$. benthamiana, at an $\mathrm{OD}_{600}$ of 1 . To avoid the occurrence of cell death, leaves were harvested already at $1 \mathrm{dpi}$, and after IP with GFP-affinity beads, the proteins were detected by IB (upper panel). The Rubisco band in the total protein reflects the amount of total protein that was employed for the IP (input, lower panel). The experiment was repeated three times, and representative results are shown. 
$\mathrm{F}$
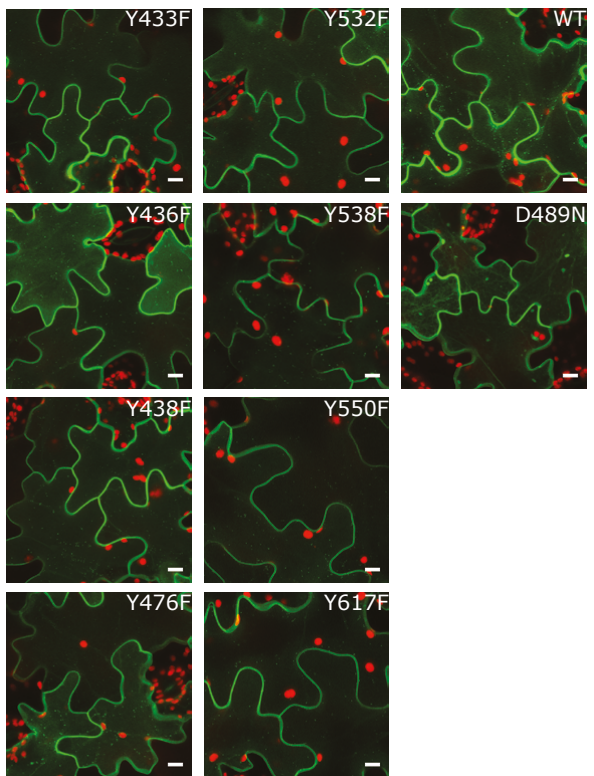

Figure S5. Additional analyses of the various AtSOBIR1 Tyr mutants (Continued). (E) Quantification of eGFP-tagged AtSOBIR1 mutant protein accumulation, as determined relative to the Rubisco band intensity of the input, as shown in panel D. Wild-type AtSOBIR1 (WT) accumulation was set to 100\%, and percentages are presented as mean +/- SE of three independent experiments. Letters indicate significant differences at $p<0.05$, as determined by one-way ANOVA, including a Tukey post hoc-test. (F) AtSOBIR1 WT, D489N, and the Tyr mutants, fused to eGFP, all localize at the PM and in cytoplasmic vesicles. Leaves of tobacco were transiently transformed with $A$. tumefaciens suspensions of $O_{600} 0.3$ driving expression of the eGFP-tagged AtSOBIR1 variants, and analysed for localization of the encoded proteins at 2 dpi by confocal microscopy. Chloroplast autofluorescence is depicted in red. White bars represent $10 \mu \mathrm{m}$. The experiments. 


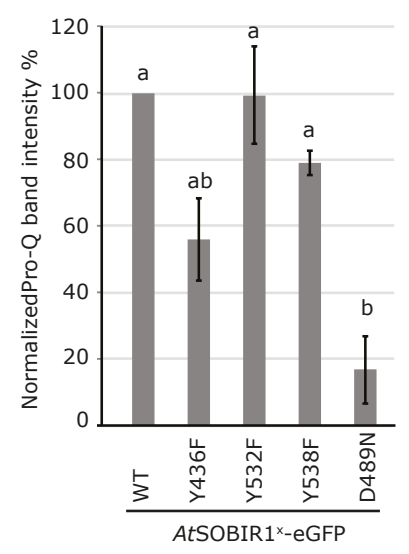

Figure S6. Quantification of the overall phosphorylation levels of three AtSOBIR1 Tyr mutants with altered functionality. Quantification of Pro-Q phosphoprotein staining of AtSOBIR1 (WT), the kinase-dead mutant (D489N), and the indicated Tyr mutants, as shown in Fig. 3C. AtSOBIR1 and AtSOBIR1 $1^{\text {Y532F }}$ show an equal overall phosphorylation level. AtSOBIR $1^{1436 \mathrm{~F}}$ appears reduced in its overall phosphorylation, whereas AtSOBIR $1^{\mathrm{YS38F}}$ seems only slightly reduced in its overall level of phosphorylation, although this is for both not significant. Ratios were obtained by dividing the band intensity of pAtSOBIR1 from the Pro-Q staining, by the band intensity reflecting the total amount of pulled-down AtSOBIR1 in the Sypro Rubystaining, shown in Fig. 3C. The ratio for wild-type AtSOBIR1 was set to $100 \%$ and percentages are presented as mean +/- SE. Letters indicate significant differences at $p<0.05$, as determined by one-way ANOVA including a Tukey post hoc-test.
A

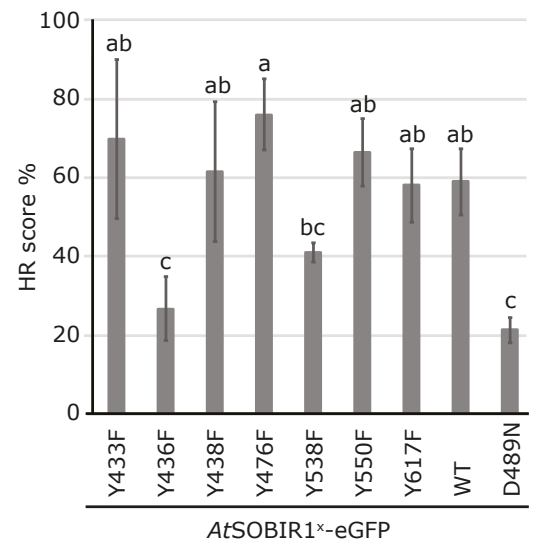

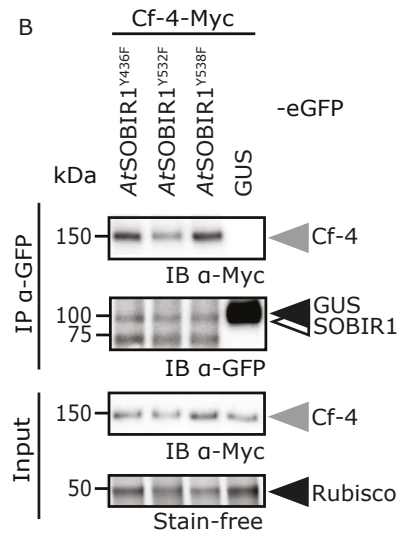

Figure S7. Y436 and Y538 are required for full SOBIR1 function downstream of Cf-4. (A) Quantification of the intensity of the HR, as shown in Fig. 3D. The HR was scored in a randomized, blind way, and was quantified as described in the Materials \& Methods section. Data are represented as mean $+/-\mathrm{SE}$, letters indicate significant differences at $\mathrm{p}<0.05$, as determined by one-way ANOVA, including a Duncan's post hoc-test. (B) AtSOBIR1 $1^{\mathrm{Y} 436 \mathrm{~F}}$, AtSOBIR1 $1^{\mathrm{Y} 532 \mathrm{~F}}$, and AtSOBIR1 ${ }^{\mathrm{Y} 538 \mathrm{~F}}$ all interact with $\mathrm{Cf}$-4. eGFP-tagged versions of the AtSOBIR1 Tyr mutants and Cf-4-Myc were transiently co-expressed in $N$. benthamiana at an $\mathrm{OD}_{600}$ of 1 , at $1 \mathrm{dpi}$ followed by IP and protein detection by IB. GUS-eGFP was included as a negative control. 
A

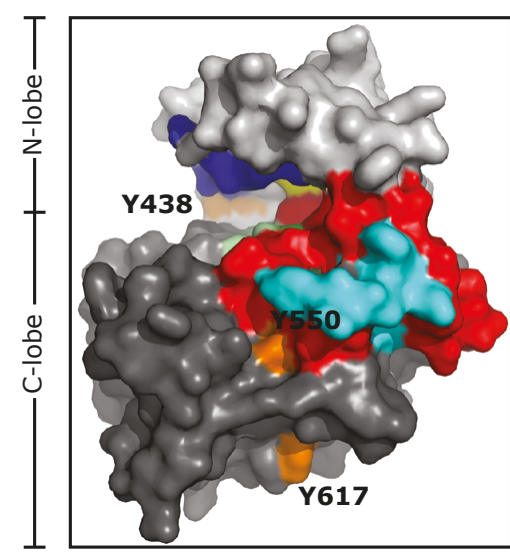

B

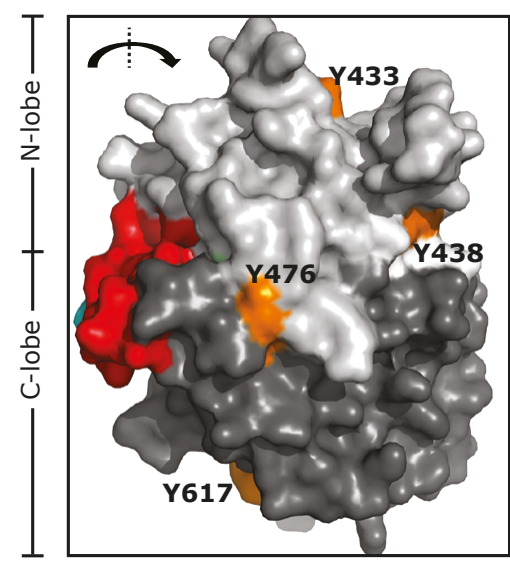

Gly-rich loop

Lys/Glu pair

Catalytic loop

Catalytic Asp

Activation segment

aEF/aF loop

Highlighted residues

Figure S8. Three dimensional model of the KD of AtSOBIR1, visualizing Y433, Y438, Y476, Y550, and Y617. (A) Surface visualization of the AtSOBIR1-KD, with Y438, Y550, and Y617 highlighted in orange. (B) Surface visualization of the AtSOBIR1-KD, rotated 180 on its vertical axis relative to panel A. Y433, Y438, Y438, Y476, and Y617 are highlighted in orange. 
A

MDQWLLGI LGFVSAFLCL I GLLLVPVSANIEGDALNALKTNLADPNNVLQSWDPTLVNPCTW FHVTCNSENSVIRVDLGNANLSGQLVPQLGQLPNLQYLELYSNN I SGKI PFELGNLTNLVSL DLYLNRLNGP I PDTLGKLQKLRFLRLNNNS LNGR I PMLLTTVASLQVLDLSNNNLAGPVPVN GSFSLFTPISFANNQLDI PPPAPPPPISPTPTSSSGVGNSATGA IAGGVAAGAALLFAAPAI LLAWWRRRKPQDHFFDVPAEEDPEVHLGQLKRFS LRELQVATDNFSNKN I LGRGGFGKVYKG RIADGSLVAVKRLKEERTQGGELQFQTEVEMI SMAV HRNLLHLLGFCMTTTERLLVYPYMAN GSVASRLRERPESDPPLEWS IRKRIALGSARGLAYLHDHCDPKI I HRDVKAANILLDEEYEA VVGDFGLAKLMDYKDTHVTTAVRGT IGH IAPEYLSTGKS SEKTDVFGYGVMLLELITGQRAF DLARLANDDDVMLLDWVKGLLKDKKYETLVDADLQGNYEEEEVEQLIRVALLCTGS SPLERP KMSEVVRMLDGDGLAERWEEWQKEEMVRQDYPAHHPHTDWI IADSTYNIRPDELSGPR

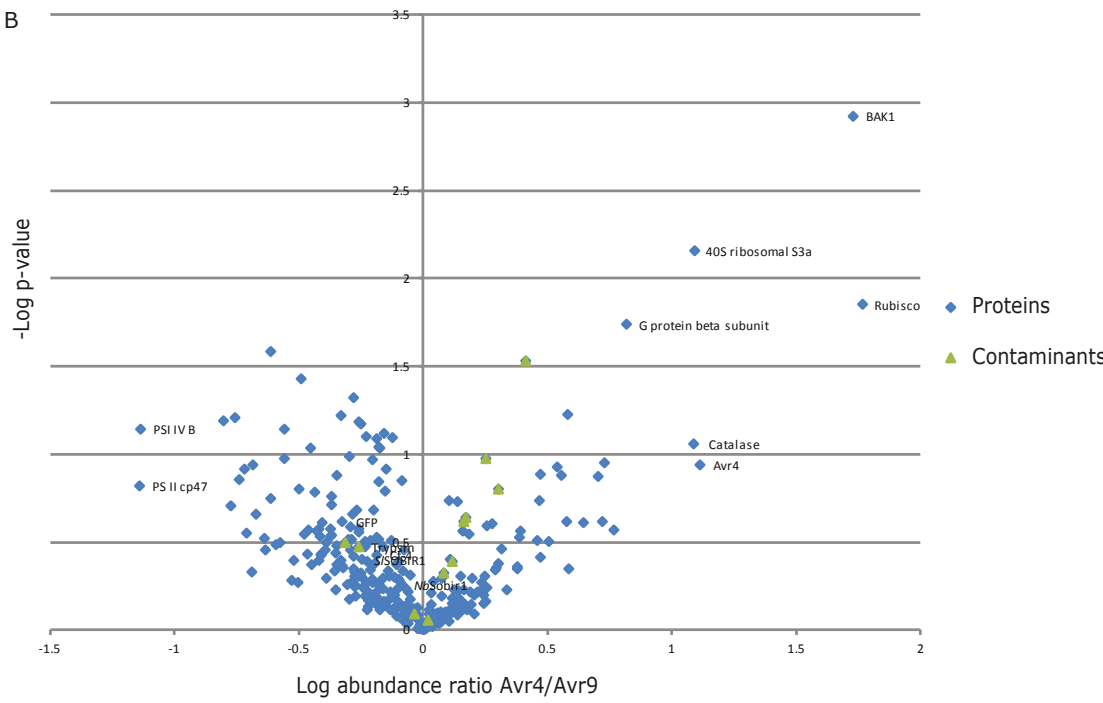

Figure S9. NbBAK1 is specifically recruited to the activated Cf-4/SOBIR1-complex. (A) Peptides from NbBAK1 homologues that are detected in samples isolated from N. benthamiana:Cf-4-eGFP plants by MS (Fig. 5B), visualised (underlined) on the sequence of NbBAK1/SERK3b (NbS00004596g0007.1). Peptides that were found only upon IP of Cf4-eGFP elicited with $5 \mu \mathrm{M}$ Avr4, are indicated in bold. (B) Volcano plot depicting the spreading of the proteins found in the MS analysis, as described in Fig. 5B. Proteins enriched in samples that were treated with Avr4 are shown on the right, and in samples that were treated with Avr9 are shown on the left. Log LFQ values of zero were replaced by a value of 4.7 (a value slightly lower than the lowest measured value) to make ratio calculations possible. Note that BAK1 is enriched in samples originating from Avr4-treated plants. 
Table S1. PCR primers for cloning of eGFP-tagged constructs. Introduced mutations are indicated with a capital, and the cacc-site for directional cloning is underlined.

\begin{tabular}{|c|c|c|}
\hline Code & Primer name * & Sequence \\
\hline ao1 & AtSOBIR1-Y433F-fw & gtcaagacccgagtgccactTcctcgtttacgagtacatgg \\
\hline ao2 & AtSOBIR1-Y433F-rv & ccatgtactcgtaaacgaggAagtggcactcgggtcttgac \\
\hline ao3 & AtSOBIR1-Y436F-fw & cgagtgccactacctcgtttTcgagtacatggaaaagggg \\
\hline ao4 & AtSOBIR1-Y436F-rv & ccccttttccatgtactcgAaaacgaggtagtggcactcg \\
\hline ao5 & AtSOBIR1-Y438F-fw & ccactacctcgtttacgagtTcatggaaaaggggagtttg \\
\hline ao6 & AtSOBIR1-Y438F-rv & caaactccccttttccatgAactcgtaaacgaggtagtgg \\
\hline ao7 & AtSOBIR1-Y476F-fw & gctgcagggcttgagtTccttcacatggatcataacc \\
\hline ao8 & AtSOBIR1-Y476F-rv & ggttatgatccatgtgaaggAactcaagccctgcagc \\
\hline ao9 & AtSOBIR1-Y532F-fw & gttgcaggtactgtgggatTcatagcaccagagttttatc \\
\hline ao10 & AtSOBIR1-Y532F-rv & gataaaactctggtgctatgAatcccacagtacctgcaac \\
\hline ao11 & AtSOBIR1-Y538F-fw & ggatacatagcaccagagttttTtcaaacccacaagttcacag \\
\hline ao12 & AtSOBIR1-Y538F-rv & ctgtgaacttgtgggtttgaAaaaactctggtgctatgtatcc \\
\hline ao13 & AtSOBIR1-Y550F-fw & cagataaatgtgatatctTtagttttggagtgattcttggg \\
\hline ao14 & AtSOBIR1-Y550F-rv & cccaagaatcactccaaaactaAagatatcacatttatctg \\
\hline ao15 & AtSOBIR1-Y617F-fw & ggttctgaagatcgcctgttTctgtacttggatgatcc \\
\hline ao16 & AtSOBIR1-Y617F-rv & ggatcatccaaagtacagAaacaggcgatcttcagaacc \\
\hline ao62 & AtSOBIR1-T519A-fw & ggcaatgccagatgcagtcGcacatattacaacctcgc \\
\hline ao63 & AtSOBIR1-T519A-rv & gcgaggttgtaatatgtgCgactgcatctggcattgcc \\
\hline ao64 & AtSOBIR1-T522A-fw & gatgcagtcacacatattGcaacctcgcatgttgcagg \\
\hline ao65 & AtSOBIR1-T522A-rv & gctgcaacatgcgaggttgCaatatgtgtgactgcatc \\
\hline ao66 & AtSOBIR1-T523A-fw & gcagtcacacatattacaGcctcgcatgttgcagg \\
\hline ao67 & AtSOBIR1-T523A-rv & cctgcaacatgcgaggCtgtaatatgtgtgactgc \\
\hline ao68 & AtSOBIR1-S524A-fw & gtcacacatattacaaccGcgcatgttgcaggtactgtgg \\
\hline ao69 & AtSOBIR1-S524A-rv & ccacagtacctgcaacatgcgCggttgtaatatgtgtgac \\
\hline ao70 & AtSOBIR1-T529A-fw & cctcgcatgttgcaggtGctgtgggatacatagcaccag \\
\hline ao71 & AtSOBIR1-T529A-rv & ctggtgctatgtatcccacagCacctgcaacatgcgagg \\
\hline ao50 & cacc-GUS-fw & Caccatggtccgtcctgtag \\
\hline ao51 & GUS-nostop-rv & Ttgtttgcctccctgctgcg \\
\hline
\end{tabular}

* fw, forward; rv, reverse 


\section{Chapter 5}

\section{RLCKs signal for immunity downstream of SOBIR1 in Solanaceous plants}

Aranka M. van der Burgh', Jim Renema', Tieme A. Helderman ${ }^{1+}$, Michael F. Seidl', Guozhi Bi², Jian-Min Zhou'2, Matthieu H. A. J. Joosten ${ }^{1}$

\footnotetext{
${ }^{1}$ Laboratory of Phytopathology, Wageningen University, Droevendaalsesteeg 1, 6708 PB Wageningen, The Netherlands

${ }^{2}$ Chinese Academy of Sciences, Center for Genome Biology, Beijing, China

${ }^{+}$Current address: Laboratory of molecular phytopathology, University of Amsterdam, Science Park 904, Amsterdam, The Netherlands
} 


\begin{abstract}
Plant cells possess transmembrane (TM)-receptors, which are either receptor-like kinases (RLKs) or receptor-like proteins (RLPs), to sense extracellular signals upon pathogen invasion. TM-receptors with an extracellular leucine-rich repeat (LRR) domain are most abundant. LRR-RLPs lack an intracellular kinase domain for downstream signalling, and therefore these RLPs constitutively interact with the LRR-RLK Suppressor Of BIR1-1 (SOBIR1) to form bimolecular RLKs. Downstream of LRR-RLKs, receptor-like cytoplasmic kinases (RLCKs) have been shown to mediate signalling in the cytoplasm. Botrytis-Induced Kinase 1 (BIK1) is a well-studied RLCK that signals downstream of LRR-RLKs, such as Flagellin-Sensing 2 (FLS2) in Arabidopsis thaliana (At). Here, we show that AtBIK1 interacts with AtSOBIR1, as well as with SOBIR1 from tomato (Solanum lycopersicum, SI). Moreover, overexpression of Solanaceous orthologues of BIK1 in Nicotiana benthamiana:Cf-4 plants enhances the hypersensitive response (HR) triggered by the LRR-RLP Cf- 4 upon recognition of the matching Cladosporium fulvum effector Avr4. A silencing screen of a broad set of $N$. benthamiana BIK1 homologues did not point to a clear Solanaceous BIK1 homologue involved in Cf-4/SOBIR1-mediated HR. However, split-luciferase assays showed the interaction of several tomato BIK1 homologues with SISOBIR1 and AtFLS2. Furthermore, in these assays, the RLCK Tomato Protein Kinase 1b (SITPK1b) was found to specifically interact with SISOBIR1. Together, our data suggest that RLCKs, in addition to facilitating signalling by LRR-RLKs, also play a role downstream of RLP/SOBIR1 complexes.
\end{abstract}




\section{Introduction}

As a prominent line of defence against pathogens, plant cells possess transmembrane (TM)-receptors that can sense extracellular pathogen signals and subsequently trigger immunity (Tang et al., 2017; Zipfel, 2014; Ranf, 2017). These TM-receptors are either receptor-like kinases (RLKs) or receptor-like proteins (RLPs). Different extracellular domains of TM-receptors can recognize different kind of ligands, and here we focus on extracellular leucine-rich repeat (LRR) domain-containing TM-receptors, which recognize proteinaceous ligands. RLKs contain a cytoplasmic kinase domain, but RLPs lack such a domain to enable downstream signalling. It is thought that RLPs therefore constitutively interact with the RLK Suppressor Of BIR11 (SOBIR1, also referred to as Evershed, EVR) to form a so-called bimolecular RLK to enable downstream signalling upon pathogen perception (Liebrand et al., 2013 and 2014; Gust \& Felix, 2014).

Downstream of RLKs receptor-like cytoplasmic kinases (RLCKs) facilitate cytoplasmic signalling for defence (Yamaguchi et al., 2013; Ma, 2014; Liang \& Zhou, 2018). RLCKs are cytoplasmic, and contain a myristoylation or palmitoylation site, which confers localization to the plasma membrane (PM). Several RLCKs have been discovered that pass the signal received from RLKs at the cell surface on to further activate downstream defence responses.

Arabidopsis thaliana (further referred to as Arabidopsis, At) carries 167 RLCKencoding genes (Lehti-Shiu et al., 2009; Shiu et al., 2004; Shiu \& Bleeker, 2001). A well-studied RLCK from Arabidopsis is Botrytis-Induced Kinase 1 (BIK1, At2g39660) (Veronese et al., 2006). AtBIK1, which is functionally redundant with its closest homologue AvrPphB Susceptible 1 (PBS1)-Like 1 (PBL1, At3g55450), interacts with several RLKs involved in defence, and plays a central role in transferring signals from these RLKs to downstream signalling partners (Zhang et al., 2010; Lu et al., 2010). For instance, BIK1 interacts with the kinase domains of Flagellin-Sensing 2 (FLS2) and the EF-TU Receptor (EFR), and is essential for FLS2 and EFR function (Zhang et al., 2010; Lu et al., 2010; Lal et al., 2018). FLS2 is an RLK that senses the flg22 peptide derived from bacterial flagellin, and upon flg22 binding to its extracellular LRR domain, forms a complex with the co-receptor Brassinosteroid-Insensitive 1 (BRI1)-Associated Kinase 1/Somatic Embryogenesis Receptor Kinase 3 (BAK1/SERK3, further referred to as BAK1) (Heese et al., 2007; Chinchilla et al., 2007; Sun et al., 2013). Upon flg22 perception, BIK1 is transphosphorylated by the activated FLS2/ BAK1 complex, and dissociates from the complex (Zhang et al., 2010; Lu et al., 2010; Xu et al., 2013; Lin et al., 2014). Subsequent signalling involves a dynamic interaction of the Respiratory Burst Oxidase Homologue D (RBOHD) enzyme, which is present at the PM, with the FLS2/BAK1/BIK1 complex (Kadota et al., 2014; Li et al., 2014). BIK1 transphosphorylates specific amino acids of RBOHD, and this transphosphorylation 
initiates the swift activation of defence responses, first of all by the production of reactive oxygen species (ROS). Defence responses are subsequently amplified by a positive feedback loop, involving additional phosphorylation events of RBOHD by calcium-dependent protein kinases (CDPKs), which requires $\mathrm{Ca}^{2+}$ (Kadota et al., 2015).

Similar to flg22 perception by FLS2, EFR perceives the elf18 peptide, which is a fragment of the bacterial microbe-associated molecular patterns (MAMP) Elongation Factor-Temperature Unstable (EF-Tu) (Zipfel et al., 2006). For EFR, transphosphorylation and dynamic interaction with BIK1 is similar as has been described for FLS2 (Zhang et al., 2010; Lu et al., 2010). Several phosphorylation sites in a loop of the BIK1 kinase that might provide an interface for protein-protein interactions were found to be essential for elf18-triggered responses upon its perception by EFR (Lal et al., 2018). Interestingly, it has recently been shown that BIK1 also provides a link to the regulation of phytohormone gene expression in the nucleus through the phosphorylation of WRKY transcription factors (Lal et al., 2018).

Initially, BIK1 was identified in a mutant screen in Arabidopsis. BIK1-knockout mutants are smaller than wild-type plants and have enhanced levels of salicylic acid (SA). They show increased basal resistance to Pseudomonas infections (Veronese et al., 2006; Zhang et al., 2010) and decreased resistance to the necrotrophic fungus Botrytis cinerea (Veronese et al., 2006). Additionally, the knockout of BIK1 in Arabidopsis compromises the activation of immune responses triggered upon treatment with MAMPs like flg22, elf18, and chitin (Zhang et al., 2010; Lu et al., 2010; Li et al., 2014). These observations reveal that BIK1 is a positive regulator of RLK-induced defence responses, and that BIK1 is possibly guarded, resulting in the constitutive activation of immune responses upon knockout of BIK1 (Veronese et al., 2006; Zhang et al., 2010; Su et al., 2018).

Besides RLCKs of Arabidopsis, RLCKs of other plant species like tomato (Solanum lycopersicum, $S l$ ) and rice (Oryza sativa, Os) have been studied. Tomato Protein Kinase 1b (SITPK1b), which is required for resistance to $B$. cinerea, has been shown to be able to rescue the BIK1-knockout phenotype in Arabidopsis (AbuQamar et al., 2008). Additionally, threonine (Thr, T) residue 238 in SITPK1b, which corresponds to T237 in AtBIK1, is a specific potential phosphorylation site in the activation loop of the kinase domain that is important for defence signalling by both RLCKs (AbuQumar et al., 2008; Lu et al., 2010). Another study shows that in rice OsRLCK176 and OsRLCK185 positively regulate chitin-triggered responses (Ao et al., 2014; Yamaguchi et al., 2013).

Next to RLCKs with a role as positive regulator of defence, there are also RLCKs that have been proposed to play a negative role in defence signalling. For example, PBS1-Like 13 (PBL13, At5g35580) is an RLCK that acts as a suppressor of defence (Lin et al., 2015). Upon knockout of $P B L 13$, plants show enhanced resistance, enhanced ROS production, and enhanced mitogen-activated protein kinase (MAPK) 3 and MAPK6 activation, as well as an elevated expression of the Pathogenesis-Related 
protein 1 (PR1) gene (Lin et al., 2015). PBL13 interacts with RBOHD in the resting state, and this association is disrupted upon treatment with flg22 (Lin et al., 2015). Kinase activity and phosphorylation of the unique repeat domain of PBL13 at its C-terminus is essential for its negative role in immune regulation (Lin et al., 2015).

RLCKs can have a positive, as well as a negative regulatory role in defence of plants against pathogens. Additionally, roles of RLCKs in plant development have been revealed (Eckardt, 2011). The crosstalk between defence and development is an interesting phenomenon (Macho \& Zipfel, 2014; Belkhadir et al., 2014). AtBIK1, for example, is a positive regulator of RLK-triggered defence responses, but a negative regulator of development (Lin et al., 2013). Brassinosteroid (BR) signalling is mediated by the RLK BRI1 (Nam \& Li, 2002), and bik 1 plants are hypersensitive to BR (Lin et al., 2013). BIK1 interacts with the kinase domain of BRI1, thereby probably suppressing BRI1 kinase activity, and upon BR-perception BRI1 transphosphorylates, and releases BIK1 (Lin et al., 2013). Heterogeneous distribution of BIK1 and other RLCKs, in different nanodomains present at the PM might account for differential regulation of downstream responses (Bücherl et al., 2017). bik 1 Arabidopsis plants are smaller, have an altered root phenotype (shorter primary roots, and longer and more root hairs and lateral roots), and flower earlier than wild-type plants (Veronese et al., 2008). Interestingly, the phosphorylation of T237 in AtBIK1 seems to be specifically involved in defence signalling and not in development, as the Thr to alanine (Ala, A) mutation of this phosphorylation site can still complement the growth phenotype, but not the defence phenotype (Lu et al., 2010; AbuQumar et al., 2008). BR-Signalling Kinases (BSKs, e.g. BSK1, At4g35230), are positive regulators of BRI1-signalling, as overexpression of BSKs activates BR-signalling (Tang et al., 2008). Interestingly, BSK1 was also found to be a positive regulator of defence signalling by FLS2 (Shi et al., 2013). Recently, BSK1 was found to link signalling from the cell surface to activation of the MAPK cascade, as BSK1 phosphorylates MAPK Kinase Kinase 5 (MAPKKK5) upon flg22 recognition by FLS2, which was found to be critical for MAMP-induced defence responses (Yan et al., 2018). Such a link was also found for the RLCK PBL27, which connects chitin recognition by the RLK Chitin Elicitor Receptor Kinase 1 (CERK1) to activation of the MAPK cascade (Yamada et al., 2016).

The signalling pathway that is activated upon pathogen recognition by RLKs has been deciphered step by step, and, as described above, includes BIK1 amongst other RLCKs as a first downstream signalling partner in the cytoplasm (Couto \& Zipfel, 2016). So far, no RLCKs essential for defence signalling downstream of RLP/ SOBIR1 complexes have been identified, although some studies indicate that RLCKs might play a role downstream of SOBIR1-containing complexes. For example, CAST AWAY (CST; At4g35600) is an Arabidopsis RLCK that interacts with SOBIR1/EVR, and inhibits organ abscission (Burr et al., 2011). However, no role of CST in regulating defence responses has been determined. Rowland and co-workers (Rowland et al., 
2005) identified Avr9/Cf-9-Induced Kinase 1 (ACIK1) as an RCLK playing a role in signalling downstream of the RLPs Cf-9 and Cf-4 in Nicotiana benthamiana (Nb), tobacco ( $N$. tabacum, $N t$ ) and tomato, conferring resistance to the fungal pathogen Cladosporium fulvum in tomato. Upon silencing of ACIK1 in several Solanaceous plants, the Cf-mediated hypersensitive response (HR) and resistance to C. fulvum were compromised, indicating that this RLCK plays a role as a positive regulator downstream of $\mathrm{Cf}$ proteins (Rowland et al., 2005).

Here we show that SOBIR1 and AtBIK1 interact in an un-elicited state, hinting to a possible role of BIK1 in signalling downstream of RLP/SOBIR1-containing complexes. We identify possible Solanaceous homologues of AtBIK1 based on phylogenetic analyses, and show that overexpression of the closest Solanaceous BIK1 homologues in $N$. benthamiana enhances the Cf-4/Avr4-induced HR. Silencing of several $N$. benthamiana BIK1 homologues, did not help to determine a role of these BIK1 homologues in the Cf-4/Avr4-triggered HR. However, interaction studies of a set of tomato BIK1 homologues revealed the interaction of several of these tomato BIK1 homologues with SOBIR1, using a split-luciferase assay. Moreover, SITPK1b appeared to specifically interact with SISOBIR1 and not with AtFLS2. Together, these results show that RLCKs also play a role in RLP/SOBIR1 bimolecular RLKs.

\section{Results \& Discussion}

\section{AtBIK1 and SOBIR1 interact in planta}

To investigate whether BIK1 is, next to its role in RLK-mediated defence signalling, also involved in RLP/SOBIR1-mediated defence signalling, we set out to examine whether SOBIR1 and AtBIK1 interact. Overexpression of HA-tagged AtSOBIR1 and FLAG-tagged AtBIK1 in Arabidopsis protoplasts and subsequent pull-down of AtBIK1FLAG, showed a clear signal for co-purifying AtSOBIR1-HA (Fig. 1A). This indicates that $A t S O B I R 1$ and AtBIK1 likely interact in planta.

To substantiate the possible SOBIR1/BIK1 interaction in planta, we over-expressed HA-tagged AtBIK1 together with GFP-tagged AtSOBIR1 in N. benthamiana leaves. Additionally, we tested for an interaction of AtBIK1 with SISOBIR1, to determine whether the interaction is conserved in Solanaceous plants. As negative and positive controls for interaction, we took along GUS and AtFLS2, respectively. Immunoblotting (IB) revealed that AtBIK1 seems to co-immunoprecipitate with both SOBIR1 orthologues, as well as with AtFLS2 (Fig. 1B), which confirmed that BIK1 and SOBIR1 interact in planta. Surprisingly, also the negative control sample with GUS-eGFP showed a faint band for BIK1, which indicates that the signal of co-purifying BIK1 can partially be explained as being non-specific background. 
A

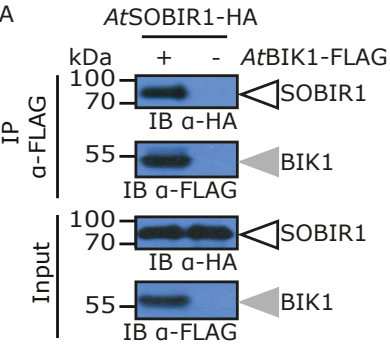

B

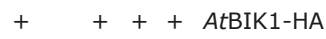

$+$

GUS
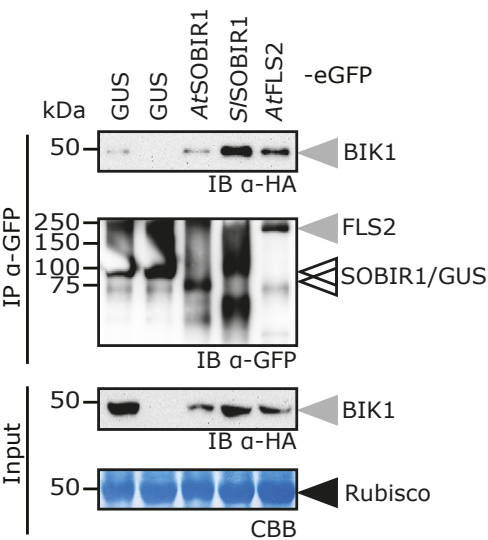

C AtBIK1-Cluc

SISOBIR1 AtFLS2 GUS -Nluc

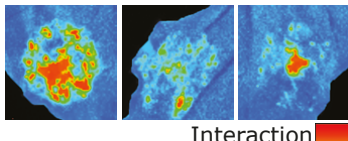

Interaction

No interaction

Figure 1. AtBIK1 and SOBIR1 interact in planta (A) AtSOBIR1-HA was transiently co-expressed with AtBIK1-FLAG in Arabidopsis protoplasts (Col-0). For this, leaves of four-week-old Arabidopsis plants were harvested and the plasmids were transfected into protoplasts. Total protein samples were extracted after $12 \mathrm{hr}$, and subjected to immunoprecipitation (IP) using FLAG affinity beads. Proteins were detected by immunoblotting (IB). Note that AtSOBIR1-HA co-purifies with AtBIK1-FLAG. (B) eGFP-tagged AtSOBIR1 and SISOBIR1 were transiently co-expressed with AtBIK1-HA and the silencing suppressor P19 in $N$. benthamiana at an $\mathrm{OD}_{600}$ of 1 for each construct. Leaves were harvested at 2 days post infiltration (dpi), followed by IP using GFP affinity beads, and protein detection by IB. GUS-eGFP was included as a negative control, and AtFLS2-eGFP served as a positive control. The Rubisco band of the input samples indicates equal loading. Note that there is interaction between AtBIK1 and SOBIR1, and that a pull-down of GUS also results in a faint AtBIK1 back-ground band. (C) Cluc-tagged AtBIK1 was transiently co-expressed in N. benthamiana:Cf-4 with Nluc-tagged SISOBIR1 and P19 using an $\mathrm{OD}_{600}$ of 0.5 per construct. Nluctagged AtFLS2 and GUS were included as positive and negative control, respectively. At 3 dpi, luciferase activity was measured using the ChemiDoc. Representative images of two independent experiments, each including six leaves, are shown. Blue indicates there is no luciferase signal, whereas a colour increasing to red indicates a stronger luciferase signal, representing a possibly stronger and/or more abundant interaction between the proteins, as in addition to variation in binding affinity the interacting proteins can also accumulate to different levels. 
To confirm the observed interaction between AtBIK1 and SISOBIR1, we employed a split-luciferase assay (Chen et al., 2008), which is a technique in which protein-protein interactions are studied in intact plants. Split-luciferase has been used in several studies to detect whether candidate proteins interact in planta (Chen et al., 2008; Li et al., 2010; Lin et al., 2015). We overexpressed AtBIK1, C-terminally tagged with the C-terminal part of the luciferase enzyme (Cluc), in combination with SISOBIR1 C-terminally tagged with the N-terminal part of the luciferase enzyme (Nluc) in N. benthamiana:Cf-4 plants. AtFLS2-Nluc and GUS-Nluc were included as positive and negative control, respectively (Fig. 1C). All tagged-proteins properly accumulate (Fig. S1A and B). Even though the negative control shows some background luminescence (Fig. $1 \mathrm{C}$, right panel) and the positive control does not show a very intense signal (Fig. 1C, middle panel), this assay further confirmed the interaction between AtBIK1 and SISOBIR1, as luciferase activity is clearly observed for this combination (Fig. 1C, left panel).

The data presented here show that AtBIK1 interacts with SOBIR1 from Arabidopsis and tomato, which suggests that BIK1 also plays a role downstream of RLP/ SOBIR1 complexes, in addition to functioning downstream of RLKs. This interaction proved to be apparent in a co-IP when using Arabidopsis protoplasts (Fig. 1A), but co-IP experiments employing agroinfiltration of $N$. benthamiana leaves were less conclusive (Fig. 1B). Also the performed split-luciferase assay shows some background illumination in the GUS control (Fig. 1C). A possible explanation of these background signals using GUS with the co-IP and split-luciferase assay could be that the GUS fusion proteins accumulate to high levels when compared to the other fusion proteins (Fig. S1). This might lead to relatively more non-specific interaction with this highly abundant protein by chance or as a result of intrinsic affinity of the Nluc domain for the Cluc domain. Additionally, infiltration of the A. tumefaciens suspensions might initiate a basal defence response of $N$. benthamiana, resulting in the dissociation of BIK1 from the kinase domain of SOBIR1, and leading to only a weak interaction signal.

Although in the future, more thorough experiments, with additional negative controls, should provide more support, together, these experiments indicate that BIK1 and SOBIR1 are likely interacting signalling partners in Arabidopsis as well as in tomato.

\section{Overexpression of AtBIK1 and also its Solanaceous orthologues affects the Cf-4/Avr4-triggered HR in N. benthamiana:Cf-4}

As SOBIR1 and AtBIK1 appear to interact in planta (Fig. 1), AtBIK1 and its Solanaceous homologues might play a role in signalling events that take place downstream of RLP/ SOBIR1 complexes. To assess this, we analysed the effect of increased BIK1 protein levels on the Cf-4/Avr4-induced HR. We overexpressed AtBIK1 and its Solanaceous orthologues from $N$. benthamiana and tomato, namely NbS00042854g0003 
and Solyc04g011520 (in short Nb42854 and S111520), respectively (Fig. S2), in N. benthamiana:Cf-4 plants, and checked for an effect on the Cf-4/Avr4-triggered HR (Gabriëls et al., 2006). We anticipated that, if BIK1 would be a signalling component downstream of the Cf-4/SOBIR1 complex as it is for RLKs like FLS2, the Cf-4/Avr4triggered $\mathrm{HR}$ might be enhanced upon overexpression of BIK1. To our surprise, co-expression of AtBIK1 with Avr4 appeared to mildly suppress the Cf-4/Avr4triggered HR (Fig. 2A and $2 \mathrm{~B}$ ). In contrast, co-expression of the two Solanaceous BIK1-orthologues with Avr4 appeared to mildly stimulate the Cf-4/Avr4-triggered $H R$. However, upon quantification of the $H R$, this stimulation appeared not to be significant (Fig. 2A and 2B). No cell death is observed upon overexpression of any of the RLCKs together with Avr9 in N. benthamiana:Cf-4 (Fig. 2A), and the various RLCK proteins were found to accumulate to similar levels (Fig. S3).

In our experiments, the overexpression of AtBIK1 suppressed the Cf-4/Avr4induced HR (Fig. 2). This observation could indicate that AtBIK1, in contrast to its positive role in FLS2/flg22-triggered defence signalling (Lu et al., 2010; Zhang et al., 2010), plays a role as a negative regulator in Cf-4/Avr4-triggered defence signalling. As mentioned earlier, next to RLCKs that function as positive regulators of defence, such as AtBIK1 in FLS2-triggered defence, there are also RLCKs that act as negative regulators of defence (Tang et al., 2017), of which an example is PBL13 (Lin et al., 2015). A recent study compared the role of BIK1 downstream of FLS2 and RLP23 in Arabidopsis (Wan, 2017). RLP23 recognizes an epitope of Necrosis and Ethyleneinducing Peptide 1 (NEP1)-Like Proteins (NLPs) (Albert et al., 2015). Interestingly, Wan and co-workers show that AtBIK1 appears to act as a negative regulator of RLP-mediated defence responses, in contrast to its role as positive regulator for RLKs (Wan, 2017). Possibly, differential phosphorylation of AtBIK1 might account for different downstream roles of this RLCK. Additionally, differential distribution of BIK1 and other RLCKs over various nanodomains might account for differential regulation of immune responses (Bücherl et al., 2017). Another explanation could be that, as we are performing heterologous overexpression in $N$. benthamiana, AtBIK1 is less capable of transferring the phosphorylation signal than the endogenous $N$. benthamiana RLCK(s), and thereby AtBIK1 might hamper defence signalling, which is observed as a suppressed HR. Interestingly, overexpression of the Solanaceous orthologues of AtBIK1 seemed to slightly stimulate the Cf-4/Avr4-triggered immune response.

Our data indicate that AtBIK1 and its $N$. benthamiana and tomato orthologue might play a role downstream of the $\mathrm{Cf}-4 / \mathrm{SOBIR} 1$ complex. Further tests, for example exploiting a possible dominant-negative effect of inactive BIK1 mutants might shed more light on the exact role of BIK1 homologues downstream in the Cf-4/ Avr4-triggered defence signalling pathway. AtBIK1 $1^{\mathrm{K} 105 \mathrm{E}}$, which lacks kinase activity, is impaired in its signalling capacity, whereas $A t B I K 1^{\top 237 A}$ is specifically impaired in 
defence signalling (Zhang et al., 2010; Lu et al., 2010; Laluk et al., 2011). Overexpression of these mutants and also their Solanaceous homologues with equivalent mutations, might suppress the Cf-4/Avr4-triggered HR. Additionally, it would be interesting to test phylogenetically more distant BIK1 homologues of $N$. benthamiana and tomato for a possible role downstream of the $\mathrm{Cf}-4 / \mathrm{SOBIR} 1$ complex, such as SIACIK1 (Rowland et al., 2005) and SITPK1b (AbuQumar et al., 2008) (Fig. S2).

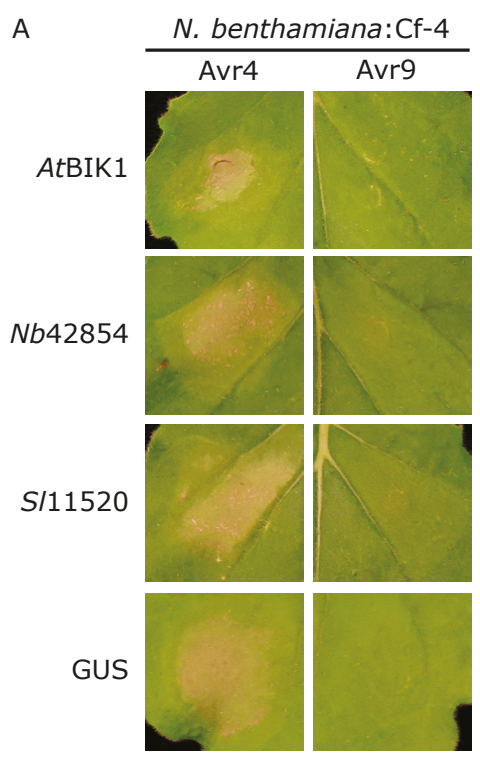

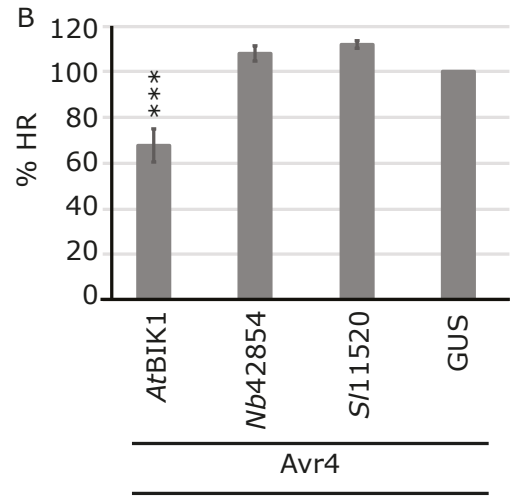

N. benthamiana:Cf-4

Figure 2. Overexpression of AtBIK1 and also its Solanaceous orthologues affects the Cf-4/Avr4triggered HR in $\mathbf{N}$. benthamiana:Cf-4. (A) The Cf-4/Avr4-induced HR appears slightly suppressed upon overexpression of $A t B I K 1$, and slightly stimulated upon overexpression of its phylogenetically closest homologues from $N$. benthamiana and tomato. C-terminally eGFP-tagged RLCKs or GUS-eGFP were transiently co-expressed with $\mathrm{P} 19$, both at an $\mathrm{OD}_{600}$ of 1 , and with Avr4 or Avr9 at an $\mathrm{OD}_{600}$ of 0.03 . The $\mathrm{HR}$ was scored at $3 \mathrm{dpi}$. The experiment was repeated five times with at least four infiltrated leaves per experiment, and representative pictures are shown. (B) Quantification of the HR shown in A. The HR was scored as described in the Materials \& Methods. The level of Cf-4/Avr4-induced HR upon co-expression with GUS-eGFP was set to $100 \%$. Percentages are presented as mean +/- SE of the five independent experiments. Note that overexpression of AtBIK1 significantly reduced the Cf-4/Avr4-triggered HR $(p<0.001)$. Overexpression of the BIK1 orthologues from $N$. benthamiana and tomato did not significantly affect the Cf-4/Avr4-triggered HR, as determined by Dunnett's t-test.

\section{Virus-induced gene silencing studies point to various roles for different $\mathbf{N}$. benthamiana BIK1 homologues}

To further study the potential roles of RLCKs downstream of the Cf-4/SOBIR1 complex in Solanaceous plants, we employed virus-induced gene silencing (VIGS) on a selection of RLCKs in N. benthamiana. Apart from the phylogenetically closest 
homologue of AtBIK1 in N. benthamiana, Nb42854, we also targeted a wide set of additional $N$. benthamiana RLCKs based on amino acid sequence and domain similarity of the encoded proteins to AtBIK1 (Fig. S2 and Table S1). In addition potentially interesting RLCKs of which a role in defence has earlier been reported were also taken along (Table S1). Unfortunately, we were not able to clone two of the selected candidates (Fig. S2).

Three weeks after inoculation of $N$. benthamiana:Cf-4 plants with recombinant TRV constructs with the aim to knockdown the expression of the various endogenous RLCKs, we transiently expressed Avr4. We monitored the appearance of an HR in the infiltrated leaf area over a period of several days. We expected to observe a suppressed HR upon successfully targeting an RLCK that functions as a positive regulator downstream of the $\mathrm{Cf}-4 / \mathrm{SOBIR} 1$ complex. However, we could not identify a clear suppression of the HR in any of the plants that were inoculated with the various TRV:RLCK constructs, including the homologues of TPK $1 b$ and ACIK 1 (Fig. $3 \mathrm{~A}$ and $3 \mathrm{~B}$, Table S1). Careful re-analysis of the DNA sequence of the RLCK fragment present in the TRV constructs showed that some might also target other homologues next to the intended RLCK (Table S1). The efficiency of silencing in this experiment was not examined by RT-PCR, but the positive control, Cf-4-silenced plants, showed a slightly compromised Avr4-induced HR, although this reduction was not statistically significant in these experiments (Fig. 3A and 3B).

A possible explanation why we did not observe a significant effect on the Cf-4/ Avr4-triggered HR upon silencing of, in most cases, individual RLCKs might be gene redundancy. The family of RLCKs is extremely large (Fig. S2) (Lehti-Shiu et al., 2009), and it is possible that silencing of one particular RLCK does not have an effect because a homologue of the gene, not targeted by the TRV construct, has a similar function. For instance, a previous study that identified the RLCK ACIK1 and showed that it plays a signalling role downstream of $\mathrm{Cf}-9$, targeted several $R L C K s$ at the same time, including Nb608, Nb11711, and several others, with the designed VIGS constructs (Rowland et al., 2005; Landeo-Villanueva, personal communication). The sequence information of the $N$. benthamiana genome to date allows us to distinguish between different RLCK homologues. Future experiments to identify RLCKs involved in Cf-4/ SOBIR1-triggered defence, should aim to target sets of related RLCKs by VIGS simultaneously, either by stacking several VIGS fragments in one constructs, or by designing VIGS fragments on conserved sequences that target several RLCKs at the same time.

Interestingly, inoculation with recombinant TRV constructs targeting Nb28219 or $\mathrm{Nb20788}$ resulted in dwarfed plants (Fig. $3 \mathrm{C}$ ). This phenotype of dwarfing hints to a role of the targeted $R L C K s$ in development and/or a role in negative regulation of defence responses, as dwarfing might be explained by a spontaneous activation of immunity (Belkhadir et al., 2014; Tang et al., 2017). For instance, in Arabidopsis the 
RLCK BSK1 associates with both BRI1 and FLS2, and is a positive regulator of BR- as well as flg22-triggered signalling (Tang et al., 2008; Shi et al., 2013). Furthermore, the knockout of BIK1 in Arabidopsis leads to increased levels of defence as a result of SA accumulation, and these plants show aberrant growth (Veronesa et al., 2006). Additionally, BIK1 is a positive regulator of MAMP-induced defence responses but a negative regulator of BR-signalling (Zhang et al., 2010; Lu et al., 2010; Lin et al., 2013). RLCKs involved in defence could be guarded, for example by cytoplasmic nucleotide-binding leucine-rich repeat receptors (NB-LRRs), which could trigger defence upon sensing a reduction in the amount of the guarded RLCK (Veronesa et al., 2006; Couto \& Zipfel., 2016; Su et al., 2018). Interestingly, Nb20788 is a close orthologue of AtPBL13 (Fig. S2), and AtPBL 13 has been shown to negatively regulate defence in Arabidopsis (Lin et al., 2015). Although the specific repeat region at the C-terminus of AtPBL13, which has been shown to be phosphorylated and important for its regulatory role (Lin et al., 2015), is not conserved in Nb20788, the dwarf phenotype of the $N$. benthamiana plants upon inoculation with recombinant TRV targeting Nb20788, hints to a role in negative regulation of defence for this PBL13 orthologue. The closest homologue of $\mathrm{Nb28219}$ for which a function has been described, is AtPBL2 (Zhang et al., 2010). AtPBL2 interacts with FLS2, is released upon flg22 elicitation, and plays a positive role in MAMP signalling (Zhang et al., 2010). To elucidate the nature of the dwarf phenotypes of the plants that were inoculated with recombinant TRV constructs targeting Nb28219 or Nb20788, it will be highly informative to determine the expression levels of defence-related genes in these plants, such as genes encoding particular Pathogenesis-Related (PR) proteins.

Overall, the silencing experiments were performed only twice and, albeit with similar results, the obtained data are not conclusive. Although the dwarf phenotypes that were observed upon inoculation with recombinant TRV constructs targeting Nb28219 or Nb20788 indicate successful silencing of the gene of interest, it is highly preferable to analyse RNA transcript levels of these genes, and phylogenetically close homologues, to determine the silencing efficiency. To prove a possible involvement of RLCKs in the Cf-4/SOBIR1-mediated defence response, further experiments aimed at silencing sets of related $R L C K s$ simultaneously are required. 
A

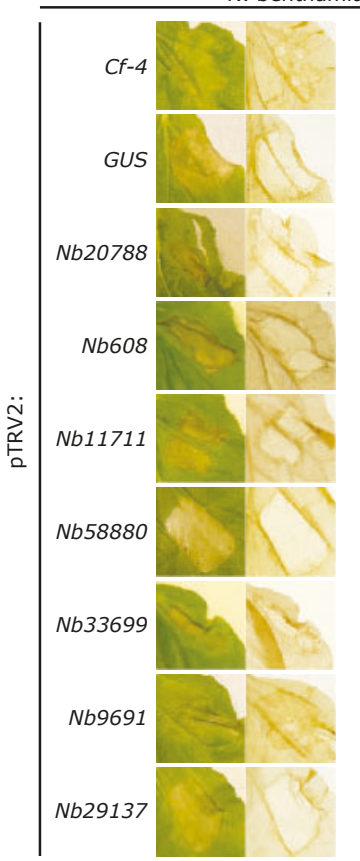

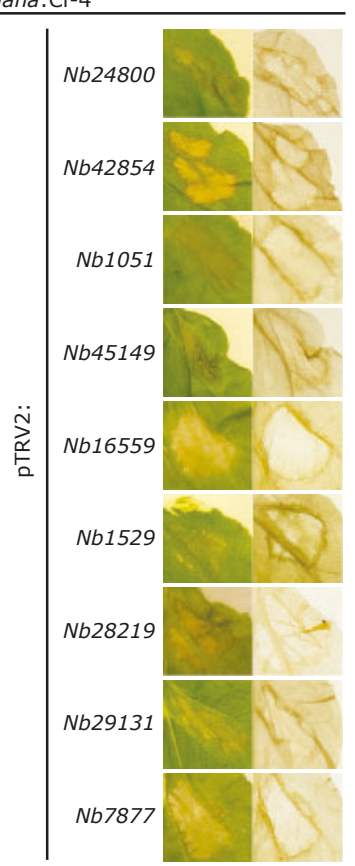

C

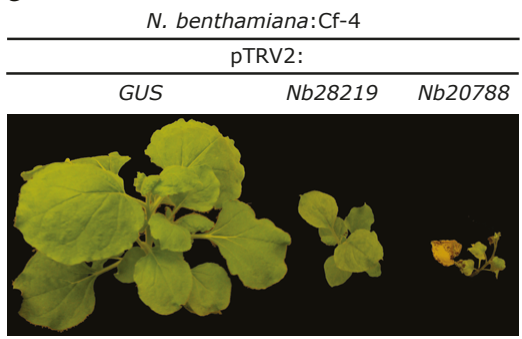

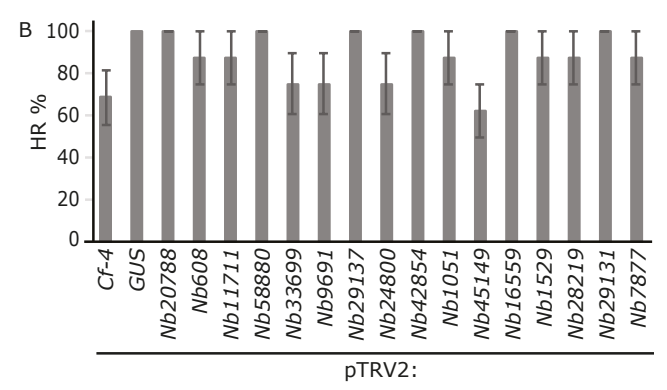

Figure 3. VIGS studies point to various roles for different $\boldsymbol{N}$. benthamiana BIK1 homologues. (A) Inoculation of $N$. benthamiana:Cf-4 plants with the indicated TRV recombinants does not lead to a clear suppression of the Cf-4/Avr4-induced HR. Three weeks after inoculation with the indicated VIGS constructs, Avr4 was agroinfiltrated at an $\mathrm{OD}_{600}$ of 0.03 . The experiment was repeated twice, with 2 plants per experiment. (B) Quantification of the HR as shown in A. The HR was scored as described in the Materials \& Methods. The level of Cf-4/Avr4-induced HR in plants inoculated with pTRV2:GUS was set to $100 \%$. Percentages are presented as mean +/- SE of two independent experiments. There was no significant difference at $\mathrm{p}<0.05$, as determined by one-way ANOVA, including a Tukey post hoc-test. (C) Inoculation of $N$. benthamiana:Cf-4 with pTRV2:Nb28219 or pTRV2:Nb20788 leads to a dwarf phenotype. Pictures were taken at 3 weeks after inoculation with the TRV recombinants. 


\section{A split-luciferase assay shows interaction between several tomato BIK1 homologues and SISOBIR1}

In a further attempt to pinpoint which Solanaceous RLCK(s) might play a role downstream of the Cf-4/SOBIR1 complex, we tested the ability of several RLCKs from tomato to interact with SISOBIR1 using a split-luciferase assay (Chen et al., 2008). We focussed on 13 tomato RLCKs, of which the expression of the encoding gene was at least 3-fold upregulated at three days after $B$. cinerea inoculation of leaflets of tomato cv. Moneymaker, as compared to healthy leaflets (J. van Kan, personal communication) (Fig. S2). These RLCKS were ordered based on their foldupregulation (number 1 showing the highest levels of upregulation and number 13 showing the lowest levels of upregulation). Unfortunately, two of the selected $R L C K S$, Solyc06g005520 (\#7) and Solyc04g011520 (\#11), could not be inserted in the splitluciferase vector due to the presence of restriction sites in the coding sequence, which were required for cloning (Fig. S2).

Subsequent overexpression of the RCLKs in N. benthamiana using agroinfiltration, showed that Solyc08g061250 (\#1) did not properly accumulate (Fig. S4). The remaining ten tomato Cluc-tagged RLCKs, as well as AtBIK1-Cluc, were analysed for their ability to interact with Nluc-tagged SISOBIR1 and AtFLS2 in planta (Fig. 4). GUS-Nluc was included as negative control. Most RLCKs tested resulted in background luminescence when expressed in combination with GUS, which can probably be explained by nonspecific interactions due to the high accumulation levels of the GUS-Nluc protein (Fig. S1). To infer interaction, we focused on the presence of increased luminescence signals when the RLCKs were co-expressed with SISOBIR1 or AtFLS2, as compared to GUS (Fig. 4).

The split-luciferase assay shows that Solyc11g062400 (S162400,\#4), Solyc01g088690 (Sl88690, \#8), Solyc05g007050 (Sl7050, \#9), and Solyc08g077560 (Sl77560, \#10) probably do not specifically interact with SISOBIR1 or AtFLS2, as the luminescence signals did not really differ from the signals upon co-expression with GUS (Fig. 4). On the contrary, Solyc07g041940 (Sl41940, \#2), Solyc04g082500 (Sl82500, \#3), Solyc06g062920 (Sl62920, \#5), Solyc05g025820 (Sl25820, \#6), and Solyc01g112220 (Sl112220, \#12) likely do specifically interact with SISOBIR1 as well as with AtFLS2, similar to AtBIK1 (Fig. 4). S/41940 (\#2) and S/25820 (\#6) are close homologues of AtPBL13 and AtRIPK (Fig. S2), which are RLCKs known to be involved in defence (Lin et al., 2015; Liu et al., 2011). Sl62920 (\#5) is a close homologue of SlACIK1 (Fig. S2), which has been shown before to play a role in Cf-mediated immunity to C. fulvum (Rowland et al., 2005). Sl112220 (\#12) in it turn is closely related to Arabidopsis CAST AWAY (AtCST) (Fig. S2), an RLCK involved in development that has been shown to interact with SOBIR1/EVR and negatively regulates cell separation signalling (Burr et al., 2011). Interestingly, Solyc06g005500 (S105500, \#13) seems to only specifically interact with 
SISOBIR1, but not with AtFLS2, as the split-luciferase signals for the combination of AtFLS2 and S105500 were as low as for the GUS control, hinting to a role of this RLCK specifically downstream of SOBIR 1 and probably not downstream of FLS2. S/05500 (\#13) has been annotated before as SITPK1b (AbuQumar et al., 2008), and it is the closest tomato homologue of AtBIK1 that we could test in the split-luciferase assay, as we were unable to clone S/11520 (\#11). N. benthamiana homologues of Sl05500 (\#13) and Sl11520 (\#11), Nb1051 and Nb42854 respectively, were included in the VIGS assays but did not cause a clear phenotype (Fig. 3A and 3B).

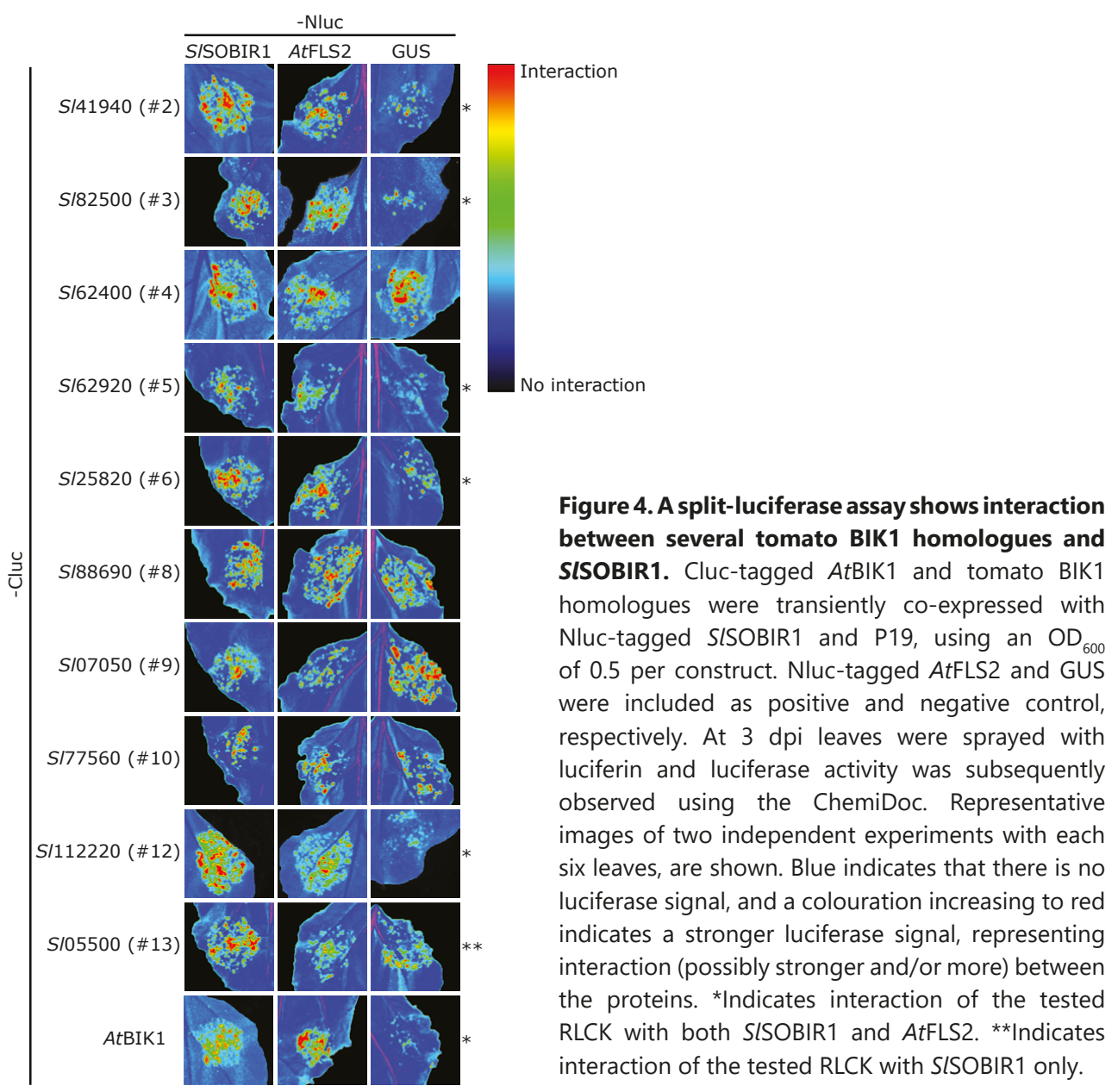


Collectively, these results show that several tomato BIK1 homologues do appear to interact with SISOBIR1. Further studies should be pursued to unravel their role in RLP/SOBIR1-mediated signalling. The interaction of AtBIK1 with AtFLS2 has been shown to become less intense upon flg22 elicitation (Zhang et al., 2010; Lu et al., 2010). Possibly, the interaction between RLCKs and SISOBIR1 is dynamic as well. Further split-luciferase assays, in which Avr4 is infiltrated with the aim to monitor whether a change in the intensity of the RLCK/SOBIR1 interaction takes place, will shed more light on the role of these RLCKs in RLP/SOPBIR1-mediated defence responses. As SOBIR1 is present in complexes with many RLPs, this assay might benefit from the use of an elicitor cocktail instead of only Avr4, to activate several RLP/SOBIR1responses simultaneously. In this way RLCK mobility might be promoted. Additionally, overexpression of the interesting RLCKs might have an effect on the Cf-4/Avr4triggered HR, as was shown for AtBIK1, Sl11520, and Nb42854 (Fig. 2).

\section{Conclusions \& Future prospects}

RLCKs play a central role in plant signalling related to defence and development (Yamagucki et al., 2013; Ma, 2014; Couto \& Zipfel, 2016; Tang et al., 2017; Liang \& Zhou, 2018). AtBIK1 has been shown to be a key downstream signalling component for RLKs (Zhang et al., 2010; Lu et al., 2010). Here, we set out to analyse whether RLCKs also play a role in immune signalling downstream of RLPs. SOBIR1 constitutively interacts with RLPs and is essential for the function of many of them (Liebrand et al., 2013; Chapter 1). The results presented here show that the RLCK AtBIK1 interacts with SOBIR1 (Fig. 1). Overexpression studies in $N$. benthamiana:Cf-4 leaves indicate that BIK1 might play a role in Cf-4/SOBIR1-mediated defence signalling (Fig. 2). Experiments involving VIGS of various RLCKS of $N$. benthamiana, up till now unfortunately did not lead to the identification of specific Solanaceous RLCKs involved in defence signalling by the Cf-4/ SOBIR1 complex (Fig. 3). However, interaction studies employing the split-luciferase system, which detects protein-protein interactions in intact plants, show that five tomato BIK1 homologues likely interact with SISOBIR1 and AtFLS2, and one, namely SITPK1b, appears to specifically interact with SISOBIR1 only (Fig. 4).

The identification of specific RLCKs downstream of RLP/SOBIR1 complexes will help us to further unravel the chain of events that take place upon the initiation of defence signalling. Interestingly, extra-large $\mathrm{G}$ protein 2 (XLG2) has recently been shown to interact with BIK1 in Arabidopsis (Liang et al., 2016; Liang et al., 2018). XLG2 was shown to block BIK1 degradation in non-activated receptor complexes, and is phosphorylated by BIK1 upon flg22 treatment to positively regulate the RBOHDdependent ROS burst (Liang et al., 2016). As G proteins have earlier been noted to be involved in SOBIR1-mediated signalling (Liu et al., 2013), it would be interesting 
to unravel this part of the signalling pathway for $\mathrm{Cf}-4 / \mathrm{SOBIR} 1$-containing complexes.

From the data that we present here, we can unfortunately not conclude with confidence which Solanaceous RLCKs are involved in Cf-4/SOBIR1-triggered defence signalling. Future research will aim to unravel which particular RLCK(s) is/are essential in SOBIR1-mediated immunity, and how these RLCKs activate immunity.

\section{Materials \& Methods}

\section{Construction of binary vectors for Agrobacterium-mediated transformation (agroinfiltration) and virus-induced gene silencing (VIGS)}

The constructs pBIN-KS-35S::AtSOBIR1-eGFP and pBIN-KS-35S::SISOBIR1-eGFP have been described previously (Liebrand et al., 2012 and 2013). P19 (Voinnet et al., 2015), pBIN61-GUS, pTRV1 (Liu et al., 2002a and 2002b), pTRV2:GUS (Tameling \& Baulcombe, 2007), pTRV2:Cf-4 (Gabriels et al., 2006), AtBIK1-HA (Zhang et al., 2010), AtBIK1-eGFP (Couto et al., 2016), and AtFLS2-eGFP (Robatzek et al., 2006) have also been described elsewhere. Avr4 and Avr9 were transiently expressed using pMOG800 constructs (van der Hoorn et al., 2000). The construction of pBIN-KS-35S::GUS-eGFP was described in chapter 4 (this thesis). The construction of Puc19-AtBIK1-FLAG was previously described (Zhang et al., 2010). For Puc19-AtSOBIR1-HA, the coding DNA sequence of AtSOBIR1 was amplified from Arabidopsis CDNA, and inserted into PUC19-HA by digestion and ligation. Generation of the AtBIK1-Cluc (Sol6625) and AtFLS2-Nluc (Sol6626) constructs for split-luciferase assays was described previously (Li et al., 2014).

To transiently overexpress putative $N$. benthamiana and tomato functional homologues of AtBIK1, we cloned NbS00042854g0003 (in short Nb42854) from cDNA obtained from $\mathrm{N}$. benthamiana plants stably expressing $\mathrm{Cf}-4$, that were agroinfiltrated with Avr4, and Solyc04g011520 (in short S111520) from cDNA obtained from Cf-4/ Avr4 tomato dying seedlings (Etalo et al., 2013) (see Table S2). After confirmation of the inserts by sequencing in pENTR/D-Topo (Invitrogen), the inserts were introduced into pBIN-KS-35S::GWY-eGFP (Sol 2095, for C-terminal tagging with eGFP) by an LR reaction using Gateway LR Clonase enzyme mix II (Invitrogen), to obtain binary vectors for in planta expression by agrobacterium-mediated transformation (agroinfiltration). The resulting binary vectors pBIN-KS-35S::Nb42854-eGFP (Sol 5179), and pBIN-KS35S::Sl11520-eGFP (Sol 5181) were transformed to Agrobacterium tumefaciens strain $\mathrm{C} 58 \mathrm{C} 1$, carrying the $\mathrm{pCH} 32$ helper plasmid.

To knockdown the expression of putative $B I K 1$ homologues from $N$. benthamiana by virus-induced gene silencing (VIGS), we cloned fragments of 150-250 bp from CDNA obtained from $N$. benthamiana plants stably expressing $\mathrm{Cf}-4$, that were agroinfiltrated with Avr4 (see Table S3). We were unable to amplify Nb5787 and 
Nb37085. The introduction of the restriction site Xbal at the $5^{\prime}$-end, and the Acc65ISpel double restriction site at the 3 '-end allowed cloning of the fragments into pTRV2 (Liu et al., 2002a and 2002b). This resulted in pTRV2-Nb165591 (Sol5534), pTRV2-Nb9691 (Sol5535), pTRV2-Nb29137 (Sol5536), pTRV2-Nb28219 (Sol5537), pTRV2-Nb58880 (Sol5538), pTRV2-Nb20788 (Sol5539), pTRV2-Nb7877 (Sol5540), pTRV2-Nb24800 (Sol5541), pTRV2-Nb29131 (Sol5542), pTRV2-Nb1529 (Sol5543), pTRV2-Nb42854 (Sol5544), pTRV2-Nb45149 (Sol5545), pTRV2-Nb1051 (Sol5547), pTRV2-Nb608 (Sol5548), pTRV2-Nb11711 (Sol5549), and pTRV2-Nb33699 (Sol5550).

To clone the various tomato $B I K 1$ homologues for split-luciferase assays, the open reading frames of the encoding genes were amplified from cDNA obtained from tomato cv. Moneymaker leaves inoculated with the necrotrophic fungal pathogen Botrytis cinerea (J. van Kan, personal communication). SISOBIR1 was amplified from pENTR-SISOBIR1 (Liebrand et al., 2013), and GUS was amplified from pENTRGUS (Invitrogen). PCR reactions were performed using primers with Kpnl and Sall restriction sites for directional cloning into Cluc- and Nluc-vectors (Chen et al., 2008) (Table S4). We were unable to clone Solyc06g005520 and Solyc04g011520 due to the presence of Sall or Kpnl restriction sites in the coding regions. The Nluc-vector includes an HA-tag for additional immunoblot (IB) analyses (Jian-Min Zhou, personal communication). After confirmation by sequencing, the vectors Solyc08G061250Cluc (Sol7200), Solyc07G041940-Cluc (Sol7202), Solyc04G082500-Cluc (Sol7204), Solyc11G062400-Cluc (Sol7206), Solyc06G062920-Cluc (Sol7208), Solyc05G025820Cluc (Sol7210), Solyc01G088690-Cluc (Sol7212), Solyc05G007050-Cluc (Sol7214), Solyc08G077560-Cluc (Sol7216), Solyc01G112220-Cluc (Sol7218), Solyc06G005500Cluc (Sol7220), SISOBIR1-Nluc (Sol6766), and GUS-Nluc (Sol6793) were transformed to $A$. tumefaciens strain $\mathrm{C} 58 \mathrm{C} 1$, carrying the $\mathrm{pCH} 32$ helper plasmid.

\section{Plant growth conditions}

Wild-type $N$. benthamiana, and $N$. benthamiana stably expressing SICf-4 under its native promoter (referred to as N. benthamiana:Cf-4) (Gabriëls et al., 2006), were grown under $16 \mathrm{~h}$ of light at $25^{\circ} \mathrm{C}$ and $8 \mathrm{~h}$ darkness at $21^{\circ} \mathrm{C}$, at $\sim 75 \%$ relative humidity. Arabidopsis plants $(\mathrm{Col}-0)$ were grown in soil at $23^{\circ} \mathrm{C}$ and $70 \%$ relative humidity, with 10/14 h day/night photoperiod for 4-5 weeks.

\section{Phylogenetic analysis of the RLCKs of tomato, potato, N. benthamiana, and Arabidopsis}

To generate a phylogenetic tree of the RLCKs of $N$. benthamiana, tomato, potato (S. tuberosum), and Arabidopsis, we queried their predicted proteomes obtained from www.solgenomics.netandwww.arabidopsis.org. Whileall predicted proteinswereused for N. benthamiana, which has a poorly annotated genome, only the longest predicted 
isoform per gene was considered for tomato, potato, and Arabidopsis, therefore excluding possible smaller alternatively-spliced isoforms. Predicted proteomes were searched for predicted pfam domains using HMMER (v3.1b; gathering cut-off) (Eddy, 1998). Sequences that contain annotated pfam domains other than cytoplasmic kinases (PF00069 or PF07714) were removed, and the sequences of the annotated kinase domains were extracted from 1,194 protein sequences (domain PF07714 was taken as a lead and sequences that deviated in length from the kinase domain of AtBIK1 were removed). Sequences of extracted kinase domains were aligned using mafft (v7.271) (Katoh \& Standley, 2013), and a neighbour-joining phylogenetic tree was built from the kinase domains using quicktree (Howe et al., 2002). Subsequently, a well-supported (95\% bootstrap support) sub-clade of putative BIK1 homologues containing 142 sequences, including AtBIK1, was isolated from this guide tree and further analysed. A refined phylogenetic tree was subsequently generated using the maximum-likelihood (ML) phylogeny as implemented in RAxML (v8.0.5) (Stamatakis, 2014). To this end, the 142 extracted kinase domains were aligned using mafft. The ML phylogeny was constructed in RAxML with the WAG amino acid substitution model, accounting for rate heterogeneity using a gamma distribution. The robustness of the phylogenetic tree was assessed using 1,000 bootstrap supports, as implemented by the rapid bootstrapping algorithm of RAxML. Protein motifs were identified using the MEME software suite (v4.9.1) (Bailey et al., 2009).

\section{Virus-induced gene silencing (VIGS) in $\mathbf{N}$. benthamiana}

VIGS using TRV-based vectors was performed in N. benthamiana:Cf-4 as described previously (Liebrand et al., 2012).

\section{Agroinfiltration in $\mathbf{N}$. benthamiana}

Agroinfiltrations were essentially performed as previously described (van der Hoorn et al., 2000). Binary constructs expressing affinity-tagged-proteins were agroinfiltrated in leaves of $N$. benthamiana plants at an $\mathrm{OD}_{600}$ of 1 , in combination with the silencing suppressor P19 also at an $\mathrm{OD}_{600}$ of 1, unless indicated otherwise. Leaves were harvested for protein isolation and immunoprecipitation (IP) of tagged proteins at 2 days post infiltration (dpi), unless indicated otherwise. Agrobacterium suspensions driving expression of Avr4 or Avr9 were infiltrated at an $\mathrm{OD}_{600}$ of 0.03 to induce the Cf-4/Avr4triggered HR, with Avr9 as a negative control. Percentages of HR were quantified by visual scoring for full HR (100\%), mildly reduced HR (60\%), strongly reduced HR (30\%), or no HR (0\%). A faster HR was scored as $130 \%$. 


\section{Immunoprecipitation and IB}

Immunoprecipitations (IPs) and co-IPs of affinity-tagged proteins were performed as described previously (Liebrand et al., 2013; Zhang et al., 2010). Proteins present in total protein extracts were visualized using Coomassie Brilliant Blue (CBB) staining, or by using the stain-free method on TGX stain-free gels (Bio-Rad\#456-8085). The following antibodies were used for protein detection on western blots: $\alpha$-GFP-HRP (MACS, 130-091-833), $\alpha$-FLAG (Sigma, F3165), $\alpha$-HA-HRP (Roche, 12013819001), $\alpha$-Cluc (Sigma, L2164), $\alpha$-mouse-HRP (GE healthcare).

\section{Split-luciferase assay}

Split-luciferase assays were performed using agroinfiltration of combinations of Clucand Nluc-tagged proteins, as described before (Chen et al., 2008). At 3 dpi, leaves were imaged with the abaxial side up using the ChemiDoc (Bio-Rad). The leaves were sprayed with luciferin (1 mM luciferin (Biovision, sodium salt 7902-100), dissolved in Milli-Q, supplemented with 1/5000 (V/V) Silwet L-77 (Lehle seeds, VIS-30)). The leaves were kept in the dark for $5 \mathrm{~min}$ to inhibit autofluorescence, and luminescence was subsequently detected using the following settings: no illumination, no filter, $2 \times 2$ binning, and an exposure time of $20 \mathrm{~min}$. Also a colorimetric image was made and merged with the luciferase picture.

\section{Acknowledgements}

We thank Unifarm personnel for excellent plant care. We acknowledge Jan van Kan for providing RNA-seq data, Laurens Deurhof for technical assistance and setting up the split-luciferase assay, and Laura Terzi for helping with optimising the split-luciferase assay. We thank Sergio Landeo-Villanueva for helping with the bioinformatics analyses. A.M.v.d.B. is supported by the Netherlands Organization for Scientific Research (NWO), Earth and Life Sciences (ALW). 


\section{References}

AbuQamar S, Chai M-F, Luo H, Song F, Mengiste T. 2008. Tomato protein kinase $1 \mathrm{~b}$ mediates signaling of plant responses to necrotrophic fungi and insect herbivory. The Plant Cell 20: 1964-1983.

Albert I, Böhm H, Albert M, Feiler CE, Imkampe J, Wallmeroth N, Brancato C, Raaymakers TM, Oome S, Zhang H, Krol E, Grefen C, Gust AA, Chai J, Hedrich R, Van den Ackerveken G, Nürnberger T. 2015. An RLP23-SOBIR1-BAK1 complex mediates NLP-triggered immunity. Nature Plants 1: 15140.

Ao Y, Li Z, Feng D, Xiong F, Liu J, Li JF, Wang M, Wang J, Liu B, Wang HB. 2014. OsCERK1 and OsRLCK176 play important roles in peptidoglycan and chitin signaling in rice innate immunity. The Plant Journal 80: 1072-1084.

Bailey TL, Boden M, Buske FA, Frith M, Grant CE, Clementi L, Ren J, Li WW, Noble WS. 2009. MEME Suite: tools for motif discovery and searching. Nucleic acids research 37: W202-W208.

Belkhadir Y, Yang L, Hetzel J, Dangl JL, Chory J. 2014. The growth-defense pivot: crisis management in plants mediated by LRR-RK surface receptors. Trends in biochemical sciences 39: 447-456.

Bücherl CA, Jarsch IK, Schudoma C, Segonzac C, Mbengue M, Robatzek S, MacLean D, Ott T, Zipfel C. 2017. Plant immune and growth receptors share common signalling components but localise to distinct plasma membrane nanodomains. elife 6: e25114.

Burr CA, Leslie ME, Orlowski SK, Chen I, Wright CE, Daniels MJ, Liljegren SJ. 2011. CAST AWAY, a membrane-associated receptor-like kinase, inhibits organ abscission in Arabidopsis. Plant Physiology 156: $1837-1850$.

Chen H, Zou Y, Shang Y, Lin H, Wang Y, Cai R, Tang X, Zhou J-M. 2008. Firefly luciferase complementation imaging assay for protein-protein interactions in plants. Plant Physiology 146: 368-376.

Chinchilla D, Zipfel C, Robatzek S, Kemmerling B, Nürnberger T, Jones JD, Felix G, Boller T. 2007. A flagellin-induced complex of the receptor FLS2 and BAK1 initiates plant defence. Nature 448: 497500.

Couto D, Niebergall R, Liang $X$, Bücherl CA, Sklenar J, Macho AP, Ntoukakis V, Derbyshire $P$, Altenbach D, Maclean D, Robatzek S, Uhrig J, Menke F, Zhou J-M, Zipfel C. 2016. The Arabidopsis protein phosphatase PP2C38 negatively regulates the central immune kinase BIK1. PLOS Pathogens 12: e1005811.

Couto D, Zipfel C. 2016. Regulation of pattern recognition receptor signalling in plants. Nature Reviews Immunology 16: 537-552.

Eckardt NA. 2011. BIK1 function in plant growth and defense signaling. The Plant Cell 23: 2806-2806.

Eddy SR. 1998. Profile hidden Markov models. Bioinformatics (Oxford, England) 14: 755-763.

Etalo DW, Stulemeijer IJE, Peter van Esse H, de Vos RCH, Bouwmeester HJ, Joosten MHAJ. 2013. System-wide hypersensitive response-associated transcriptome and metabolome reprogramming in tomato. Plant Physiology 162: 1599-1617.

Gabriëls SHEJ, Takken FLW, Vossen JH, De Jong CF, Liu Q, Turk SCHJ, Wachowski LK, Peters J, Witsenboer HMA, De Wit PJGM, Joosten MHAJ. 2006. cDNA-AFLP combined with functional analysis reveals novel genes involved in the hypersensitive response. Molecular Plant-Microbe Interactions 19: 567-576.

Gust AA, Felix G. 2014. Receptor like proteins associate with SOBIR1-type of adaptors to form bimolecular receptor kinases. Current Opinion in Plant Biology 21: 104-111.

Heese A, Hann DR, Gimenez-Ibanez S, Jones AME, He K, Li J, Schroeder JI, Peck SC, Rathjen JP. 2007. The receptor-like kinase SERK3/BAK1 is a central regulator of innate immunity in plants. Proceedings of the National Academy of Sciences of the United States of America 104: 12217-12222.

Hirayama T, Oka A. 1992. Novel protein kinase of Arabidopsis thaliana (APK1) that phosphorylates tyrosine, serine and threonine. Plant molecular biology 20: 653-662.

Howe K, Bateman A, Durbin R. 2002. QuickTree: building huge Neighbour-Joining trees of protein sequences. Bioinformatics 18: 1546-1547. 
Ito T, Takahashi N, Shimura Y, Okada K. 1997. A serine/threonine protein kinase gene isolated by an in vivo binding procedure using the Arabidopsis floral homeotic gene product, AGAMOUS. Plant and Cell Physiology 38: 248-258.

Joosten MHAJ, Cozijnsen TJ, De Wit PJ. 1994. Host resistance to a fungal tomato pathogen lost by a single base-pair change in an avirulence gene. Nature 367: 384.

Kadota Y, Shirasu K, Zipfel C. 2015. Regulation of the NADPH oxidase RBOHD during plant immunity. Plant and Cell Physiology 56: 1472-1480.

Kadota Y, Sklenar J, Derbyshire P, Stransfeld L, Asai S, Ntoukakis V, Jones Jonathan D, Shirasu K, Menke F, Jones A, Zipfel C. 2014. Direct regulation of the NADPH Oxidase RBOHD by the PRRassociated kinase BIK1 during plant immunity. Molecular Cell 54: 43-55.

Katoh K, Standley DM. 2013. MAFFT multiple sequence alignment software version 7: improvements in performance and usability. Molecular Biology and Evolution 30: 772-780.

Kodama Y, Tamura T, Hirasawa W, Nakamura K, Sano H. 2009. A novel protein phosphorylation pathway involved in osmotic-stress response in tobacco plants. Biochimie 91: 533-539.

Laluk K, Luo H, Chai M, Dhawan R, Lai Z, Mengiste T. 2011. Biochemical and genetic requirements for function of the immune response regulator BOTRYTIS-INDUCED KINASE1 in plant growth, ethylene signaling, and PAMP-triggered immunity in Arabidopsis. The Plant Cell 23: 2831-2849.

Lehti-Shiu MD, Zou C, Hanada K, Shiu S-H. 2009. Evolutionary history and stress regulation of plant receptor-like kinase/pelle genes. Plant Physiology 150: 12-26.

Li L, Li M, Yu L, Zhou Z, Liang X, Liu Z, Cai G, Gao L, Zhang X, Wang Y, Chen S, Zhou J-M. 2014. The FLS2-associated kinase BIK1 directly phosphorylates the NADPH oxidase RbohD to control plant immunity. Cell Host \& Microbe 15: 329-338.

Liang X, Ding P, Lian K, Wang J, Ma M, Li L, Li L, Li M, Zhang X, Chen S. 2016. Arabidopsis heterotrimeric $G$ proteins regulate immunity by directly coupling to the FLS2 receptor. eLife 5: e13568.

Liang X, Ma M, Zhou Z, Wang J, Yang X, Rao S, Bi G, Li L, Zhang X, Chai J, Chen S, Zhou J-M. 2018. Ligand-triggered de-repression of Arabidopsis heterotrimeric $G$ proteins coupled to immune receptor kinases. Cell Research: doi:10.1038/s41422-41018-40027-41425.

Liang X, Zhou J-M. 2018. Receptor-like cytoplasmic kinases: central players in plant receptor kinasemediated signaling. Annual Review of Plant Biology 69: 267-299.

Liebrand TWH, Smit P, Abd-El-Haliem A, de Jonge R, Cordewener JHG, America AHP, Sklenar J, Jones AME, Robatzek S, Thomma BPHJ, Tameling WIL, Joosten MHAJ. 2012. Endoplasmic reticulum-quality control chaperones facilitate the biogenesis of $\mathrm{Cf}$ receptor-like proteins involved in pathogen resistance of tomato. Plant Physiology 159: 1819-1833.

Liebrand TWH, van den Berg GCM, Zhang Z, Smit P, Cordewener JHG, America AHP, Sklenar J, Jones AME, Tameling WIL, Robatzek S, Thomma BPHJ, Joosten MHAJ. 2013. Receptor-like kinase SOBIR1/EVR interacts with receptor-like proteins in plant immunity against fungal infection. Proceedings of the National Academy of Sciences of the United States of America 110: 10010-10015.

Liebrand TWH, van den Burg HA, Joosten MHAJ. 2014. Two for all: receptor-associated kinases SOBIR1 and BAK1. Trends in Plant Science 19: 123-132.

Lin W, Lu D, Gao X, Jiang S, Ma X, Wang Z, Mengiste T, He P, Shan L. 2013. Inverse modulation of plant immune and brassinosteroid signaling pathways by the receptor-like cytoplasmic kinase BIK1. Proceedings of the National Academy of Sciences of the United States of America 110: 12114-12119.

Lin W, Li B, Lu D, Chen S, Zhu N, He P, Shan L. 2014. Tyrosine phosphorylation of protein kinase complex BAK1/BIK1 mediates Arabidopsis innate immunity. Proceedings of the National Academy of Sciences of the United States of America 111: 3632-3637.

Lin Z-JD, Liebrand TW, Yadeta KA, Coaker GL. 2015. PBL13 is a serine/threonine protein kinase that negatively regulates Arabidopsis immune responses. Plant Physiology 169: 2950-2962.

Liu J, Ding P, Sun T, Nitta Y, Dong O, Huang X, Yang W, Li X, Botella JR, Zhang Y. 2013. Heterotrimeric $G$ proteins serve as a converging point in plant defense signaling activated by multiple receptor-like kinases. Plant Physiology 161: 2146-2158. 
Liu J, Elmore JM, Lin Z-JD, Coaker G. 2011. A receptor-like cytoplasmic kinase phosphorylates the host target RIN4, leading to the activation of a plant innate immune receptor. Cell Host \& Microbe 9: 137-146.

Liu Y, Schiff M, Dinesh-Kumar S. 2002a. Virus-induced gene silencing in tomato. The Plant Journal 31: 777-786.

Liu Y, Schiff M, Marathe R, Dinesh-Kumar S. 2002b. Tobacco Rar1, EDS1 and NPR1/NIM1 like genes are required for $\mathrm{N}$-mediated resistance to tobacco mosaic virus. The Plant Journal 30: 415-429.

Lu D, Wu S, Gao X, Zhang Y, Shan L, He P. 2010. A receptor-like cytoplasmic kinase, BIK1, associates with a flagellin receptor complex to initiate plant innate immunity. Proceedings of the National Academy of Sciences of the United States of America 107: 496-501.

Ma W. 2014. From pathogen recognition to plant immunity: BIK1 cROSses the divide. Cell Host \& Microbe 15: 253-254.

Macho AP, Zipfel C. 2014. Plant PRRs and the activation of innate immune signaling. Molecular Cell 54: 263-272.

Moran TV, Walker JC. 1993. Molecular cloning of two novel protein kinase genes from Arabidopsis thaliana. Biochimica et Biophysica Acta (BBA)-Gene Structure and Expression 1216: 9-14.

Nam KH, Li J. 2002. BRI1/BAK1, a receptor kinase pair mediating brassinosteroid signaling. Cell 110: 203-212.

Ranf S. 2017. Sensing of molecular patterns through cell surface immune receptors. Current Opinion in Plant Biology 38: 68-77.

Robatzek S, Chinchilla D, Boller T. 2006. Ligand-induced endocytosis of the pattern recognition receptor FLS2 in Arabidopsis. Genes \& development 20: 537-542.

Rowland O, Ludwig AA, Merrick CJ, Baillieul F, Tracy FE, Durrant WE, Fritz-Laylin L, Nekrasov V, Sjölander K, Yoshioka H. 2005. Functional analysis of Avr9/Cf-9 rapidly elicited genes identifies a protein kinase, $\mathrm{ACIK1}$, that is essential for full $\mathrm{Cf}$-9-dependent disease resistance in tomato. The Plant Cell 17: 295-310.

Shi H, Shen Q, Qi Y, Yan H, Nie H, Chen Y, Zhao T, Katagiri F, Tang D. 2013. BR-SIGNALING KINASE1 physically associates with FLAGELLIN SENSING2 and regulates plant innate immunity in Arabidopsis. The Plant Cell 25: 1143-1157.

Shiu S-H, Bleecker AB. 2001. Plant receptor-like kinase gene family: diversity, function, and signaling. Science Signaling 2001: 22.

Shiu S-H, Karlowski WM, Pan R, Tzeng Y-H, Mayer KF, Li W-H. 2004. Comparative analysis of the receptor-like kinase family in Arabidopsis and rice. The Plant Cell 16: 1220-1234.

Stamatakis A. 2014. RAxML version 8: a tool for phylogenetic analysis and post-analysis of large phylogenies. Bioinformatics 30: 1312-1313.

Su J, Spears BJ, Kim SH, Gassmann W. 2018. Constant vigilance: plant functions guarded by resistance proteins. The Plant Journal 93: 637-650.

Sun Y, Li L, Macho AP, Han Z, Hu Z, Zipfel C, Zhou JM, Chai J. 2013. Structural basis for flg22-induced activation of the Arabidopsis FLS2-BAK1 immune complex. Science 342: 624-628.

Tameling WIL, Baulcombe DC. 2007. Physical association of the NB-LRR resistance protein Rx with a Ran GTPase-activating protein is required for extreme resistance to Potato virus X. The Plant Cell 19: $1682-1694$.

Tang D, Wang G, Zhou J-M. 2017. Receptor kinases in plant-pathogen interactions: more than pattern recognition. The Plant Cell 29: 618-637.

Tang W, Kim T-W, Oses-Prieto JA, Sun Y, Deng Z, Zhu S, Wang R, Burlingame AL, Wang Z-Y. 2008. BSKs mediate signal transduction from the receptor kinase BRI1 in Arabidopsis. Science 321: 557-560.

Thomas CM, Jones DA, Parniske M, Harrison K, Balint-Kurti PJ, Hatzixanthis K, Jones JD. 1997. Characterization of the tomato $\mathrm{Cf}-4$ gene for resistance to Cladosporium fulvum identifies sequences that determine recognitional specificity in Cf-4 and Cf-9. The Plant Cell 9: 2209-2224. 
van der Hoorn RAL, Laurent F, Roth R, de Wit PJGM. 2000. Agroinfiltration is a versatile tool that facilitates comparative analyses of Avr9/Cf-9-induced and Avr4/Cf-4-induced necrosis. Molecular Plant-Microbe Interactions 13: 439-446.

Veronese P, Nakagami H, Bluhm B, AbuQamar S, Chen X, Salmeron J, Dietrich RA, Hirt H, Mengiste T. 2006. The membrane-anchored BOTRYTIS-INDUCED KINASE1 plays distinct roles in Arabidopsis resistance to necrotrophic and biotrophic pathogens. The Plant Cell 18: 257-273.

Voinnet O, Rivas S, Mestre P, Baulcombe DC. 2015. Retraction: An enhanced transient expression system in plants based on suppression of gene silencing by the p19 protein of tomato bushy stunt virus (retraction of The Plant Journal, vol 33, pg 949, 2003).

Wan W-L. 2017. Comparative analysis of signaling pathways triggered by different pattern-recognition receptor-types. PhD thesis, University of Tübingen.

Xu J, Wei X, Yan L, Liu D, Ma Y, Guo Y, Peng C, Zhou H, Yang C, Lou Z, Shui W. 2013. Identification and functional analysis of phosphorylation residues of the Arabidopsis BOTRYTIS-INDUCED KINASE1. Protein \& Cell 4: 771-781.

Yamada K, Yamaguchi K, Shirakawa T, Nakagami H, Mine A, Ishikawa K, Fujiwara M, Narusaka M, Narusaka Y, Ichimura K. 2016. The Arabidopsis CERK1-associated kinase PBL27 connects chitin perception to MAPK activation. The EMBO Journal 35: 2468-2483.

Yamaguchi K, Yamada K, Kawasaki T. 2013. Receptor-like cytoplasmic kinases are pivotal components in pattern recognition receptor-mediated signaling in plant immunity. Plant signaling \& behavior $\mathbf{8}$ : e25662.

Yan H, Zhao Y, Shi H, Li J, Wang Y, Tang D. 2018. BRASSINOSTEROID-SIGNALING KINASE1 phosphorylates MAPKKK5 to regulate immunity in Arabidopsis. Plant Physiology 176: 2991-3002.

Zhang J, Li W, Xiang T, Liu Z, Laluk K, Ding X, Zou Y, Gao M, Zhang X, Chen S, Mengiste T, Zhang Y, Zhou JM. 2010. Receptor-like cytoplasmic kinases integrate signaling from multiple plant immune receptors and are targeted by a Pseudomonas syringae effector. Cell Host \& Microbe 7: 290-301.

Zipfel C. 2014. Plant pattern-recognition receptors. Trends in immunology 35: 345-351. 


\section{Supplemental data}
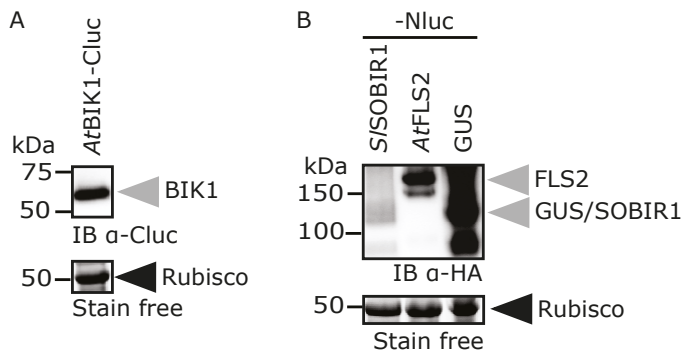

Figure S1. Cluc- and Nluc-tagged proteins properly accumulate upon their overexpression in $\boldsymbol{N}$. benthamiana. (A) Cluc- and (B) Nluc-tagged proteins were transiently expressed with P19 in N. benthamiana at an $\mathrm{OD}_{600}$ of 0.5 for each construct. Leaves were harvested at $2 \mathrm{dpi}$, followed by total protein isolation, and protein detection by IB. The Rubisco band in the samples indicates equal loading. 


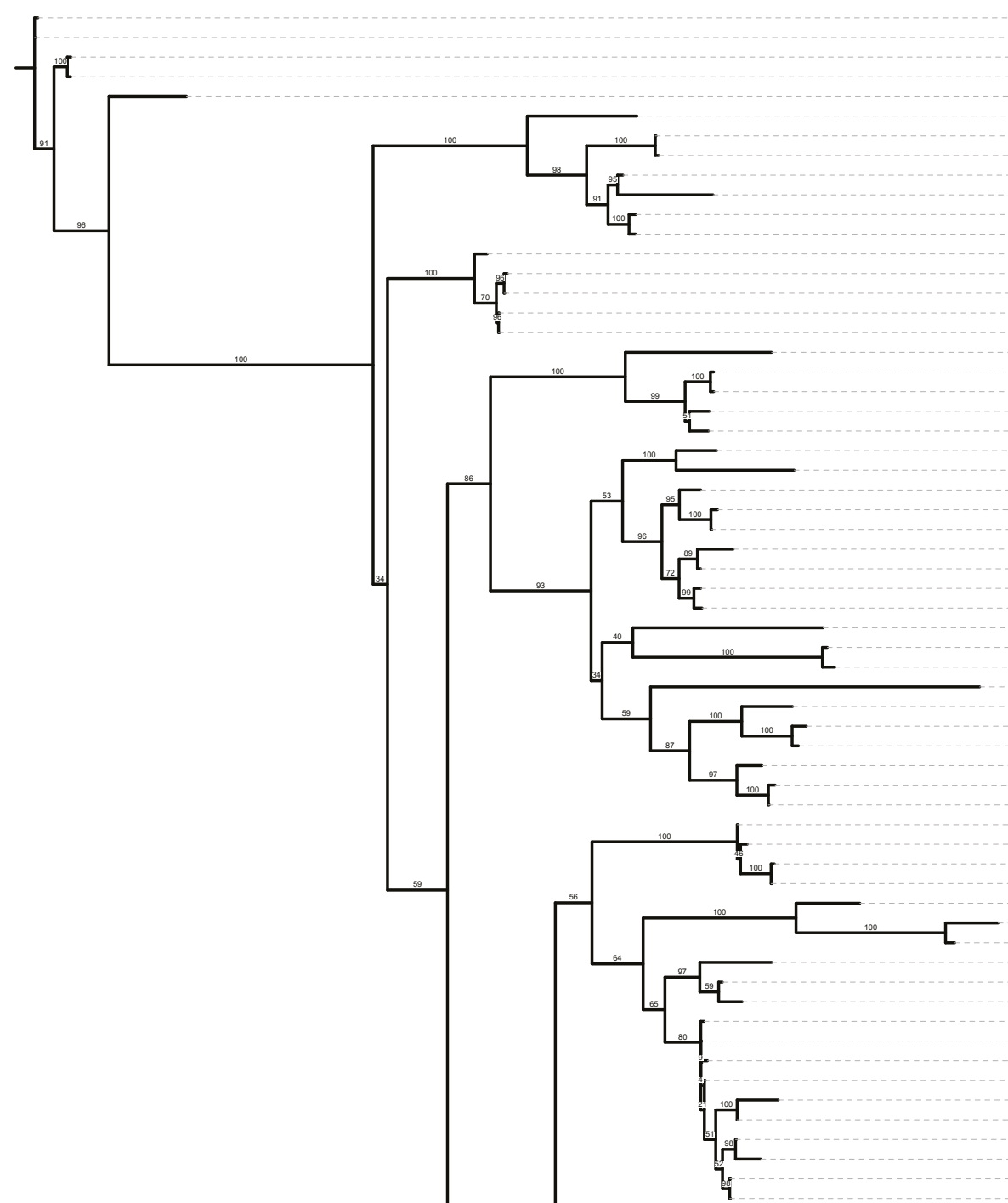

Figure S2. Phylogenetic tree of AtBIK1 and its homologues in Arabidopsis, tomato, potato, and $N$. benthamiana. (continued on next pages)

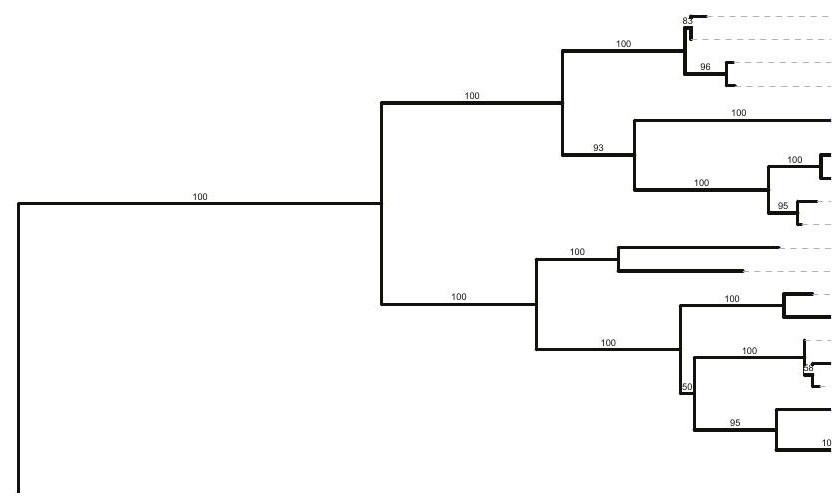


- Solyc01g028830.2.1 - - PGSC0003DMP400041518 -NbS00020322g0005.1 - - NbS00005183g0013.1 - AT5G56460.1

AT2G07180.1 _...... PGSC0003DMP400005217 - Solyc12g009310.1.1 NbS00046910g0007.1 NbS00040223g0007.1 - - PGSC0003DMP400023975 Solyc07g042590.2.1 AT5G01020.1 -

Solyc10g074710.1.1 PGSC0003DMP400048160 - NbS00033064g0016.1 NbS00017687g0003.1 - AT1G61590.1 _. - . PGSC0003DMP40002754 Solyc08g074980.2.1 - - NbS00011665g0016.1 -

AtACIK12/RIPK NbS00011463g0003.1 -

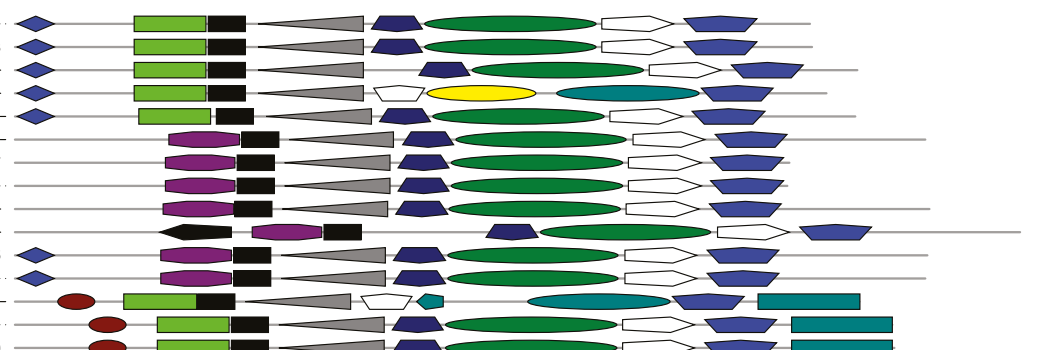

Dwarf - NbS00020788 00121

PGSC0003DMP400048503

\#2 - Solyc07g041940.2.1

\#6- Solyc05g025820.2.1

NtACIK1 NbS00000608g0110.1 NbS00000608g0110.1 --

AtPBL12 - AT2G26290.1 - -

\#1- Solyc08g061250.2.1 - PGSC0003DMP400023028 NbS00032837g0003.1 Solyc12g049360.1.1 PGSC0003DMP400007758 NbS00058880g0009.1 --

\#5 SIACIK1- Solyc06g062920.2.1 --- NbS00035481g0006.1 - NbS00017971g0004.1 - Solyc07g007980.2.1 PGSC0003DMP400053808 - NbS00000843g0010.1 - Solyc02g087830.2.1 PGSC0003DMP400002555 AT3G28690.2 AT5G15080.1 AT3G01300.1 NbS00037717g0009.1 NbS00040588g0002.1 -PGSC0003DMP40003078

\#9-Solyc05g007050.2.1 -NbS00003177g0113.1 -NbS00010113g0012.1 - NbS00016824g0009.1 NbS00001581g0008.1 Solyc01g010780.2.1 - - - PGSC0003DMP400001623 - NbS00033094g0002.1 - NbS00003125g0001.1 PGSC0003DMP400003094

\#8 Solyc019088690.2.1 - AT5G47070.1

\#10 Solyc08g077560.2.1 PGSC0003DMP400003316 NbS00058568g0003.1 NbS00021752g0009.1 AT2G28940.2

\#7- Solyc06g005520.21

AtPCRK1 AT3G09830.1 AtPCRK2 - AT5G03320.1 PGSC0003DMP400037357 NbS00004124g0020.1 - Solyc09g008010.2.1 - AT2G39110.1 - Solyc09g075720.2.1 NbS00020972g0001.1 NbS00005251g0104.1 - -
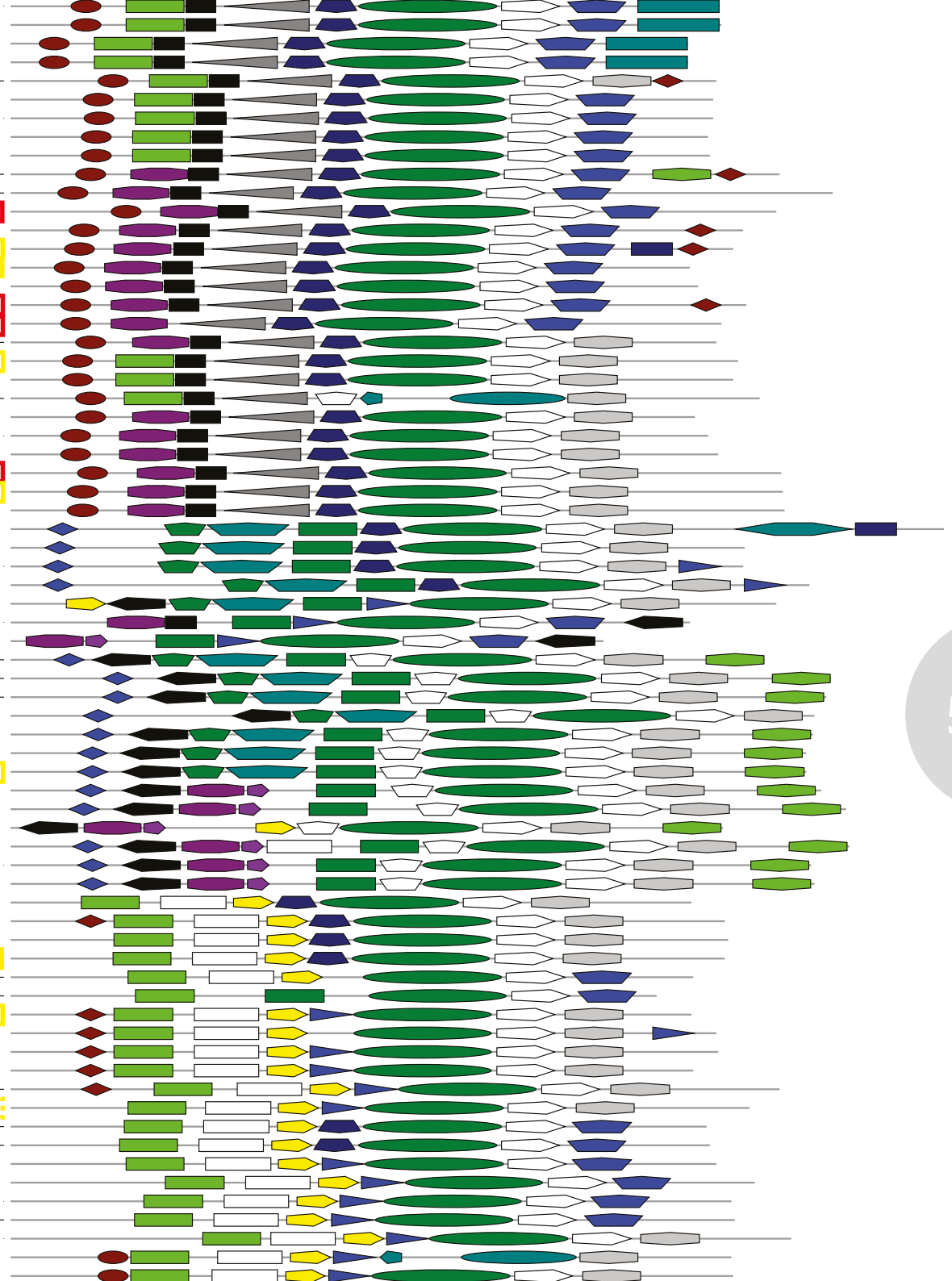
Protein motifs

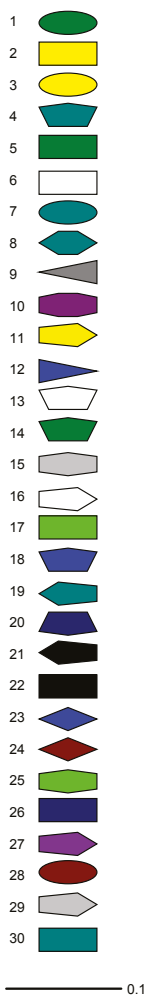

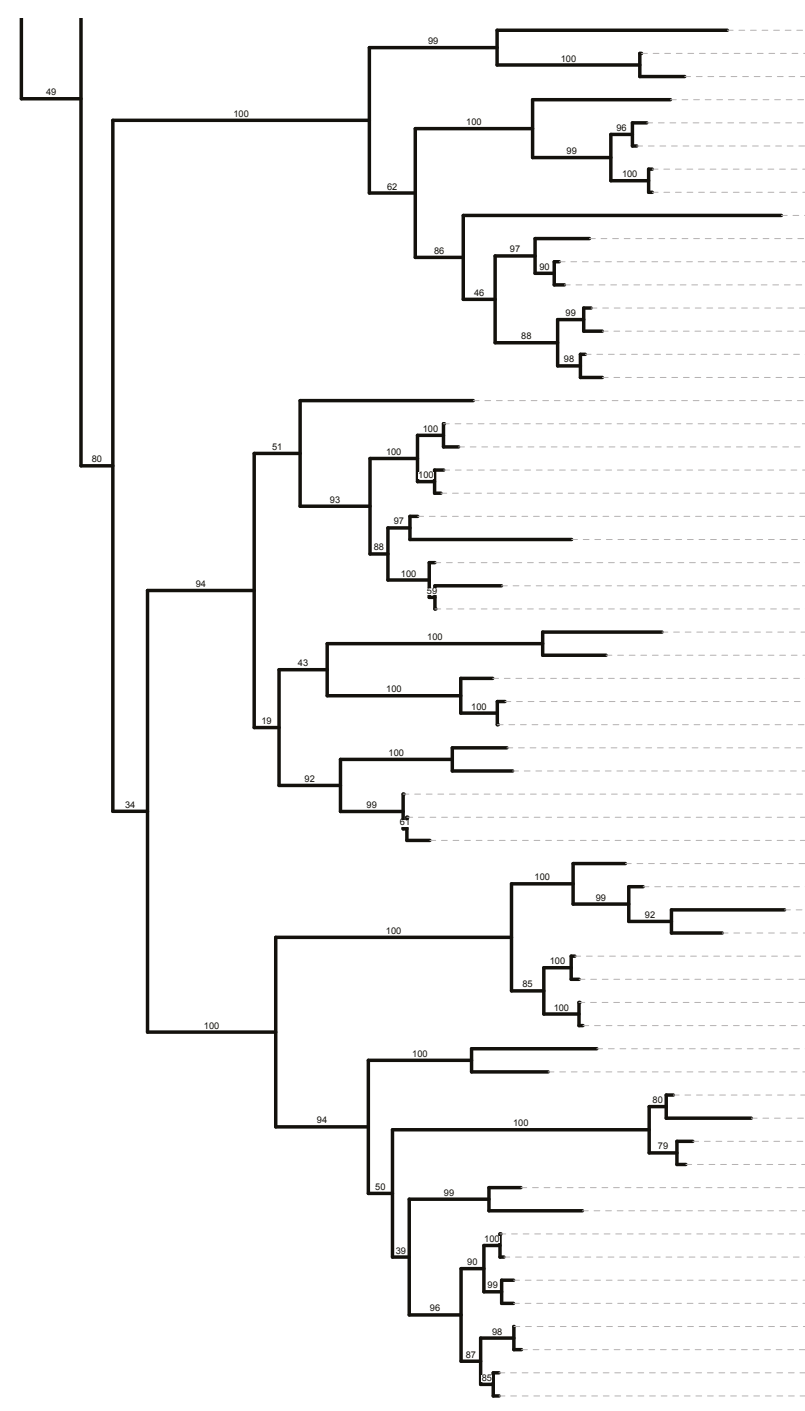




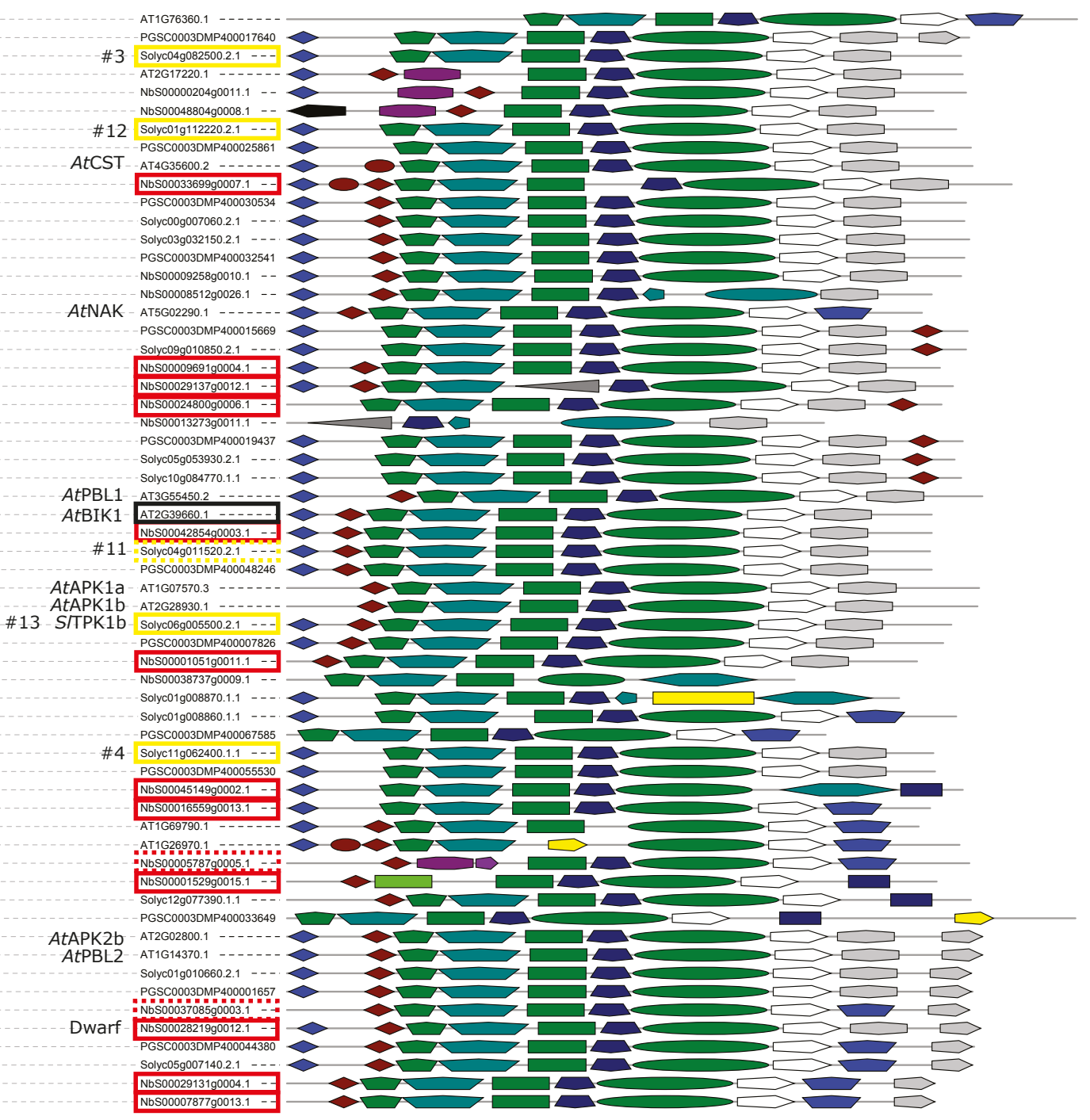

Figure S2. Phylogenetic tree of AtBIK1 and its homologues in Arabidopsis, tomato, potato, and $\mathbf{N}$. benthamiana (Continued). AtBIK1 is indicated with a black box. Homologous proteins were selected as described in the Materials \& Methods section. Bootstrap values are indicated. The branch length represents the genetic change. $N$. benthamiana RLCKs selected for VIGS of their encoding genes are indicated with a red box. 'Dwarf' indicates a dwarf phenotype upon VIGS of this gene. Tomato RLCKs of which the expression of the encoding gene was at least 3 -fold upregulated upon inoculation with $B$. cinerea were selected for interaction studies employing split-luciferase assays and are indicated with a yellow box. Indicated \#numbers are based on fold-upregulation upon B. cinerea inoculation, \#1 showing the highest levels of upregulation and \#13 showing the lowest levels of upregulation. A dotted box indicates that this RLCK was selected, but the encoding CDNA could not be cloned. 


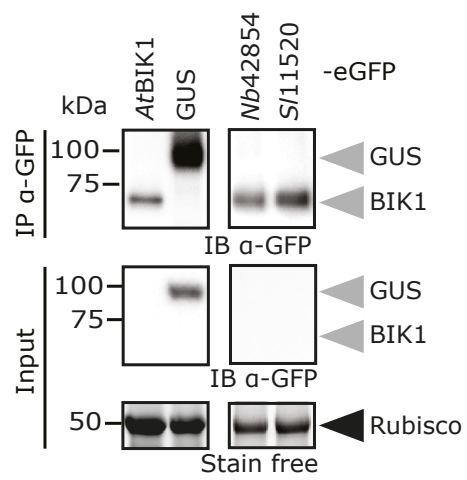

Figure S3. AtBIK1 and its Solanaceous orthologues properly accumulate upon overexpression in $\mathbf{N}$. benthamiana:Cf-4. The RLCKs with a C-terminal eGFP-tag and GUS-eGFP properly accumulate when transiently coexpressed with P19 in N. benthamiana at an $\mathrm{OD}_{600}$ of 0.5 for each construct. Leaves were harvested at $2 \mathrm{dpi}$, followed by IP using GFP affinity beads, and protein detection by IB. The Rubisco band in the input samples indicates equal loading.

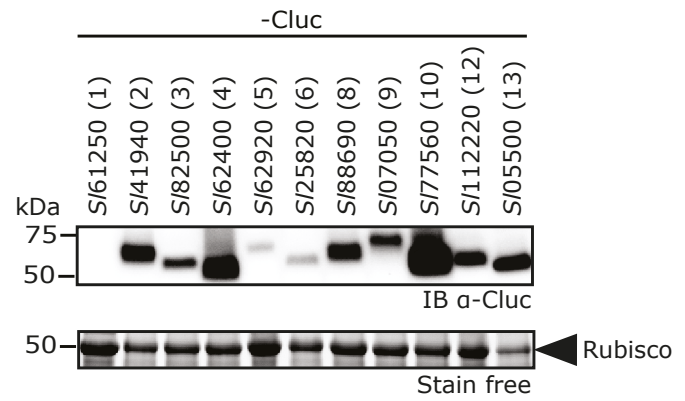

Figure S4. The 10 Cluc-tagged tomato BIK1 homologues properly accumulate. Cluc-tagged tomato BIK1 homologues were transiently expressed with P19 in N. benthamiana at an $\mathrm{OD}_{600}$ of 0.5 for each construct. Leaves were harvested at $2 \mathrm{dpi}$, followed by total protein isolation, and protein detection by IB. The Rubisco band in the samples indicates equal loading. 
Table S1. Genes encoding various RLCKs of $\boldsymbol{N}$. benthamiana targeted by VIGS.

\begin{tabular}{|c|c|c|c|c|}
\hline Gene name & $\begin{array}{l}\text { Shortened } \\
\text { gene name }\end{array}$ & $\begin{array}{l}\text { Off-target } \\
\text { NbRLCK }\end{array}$ & $\begin{array}{l}\text { Closest } \\
\text { homologue(s) }\end{array}$ & Reference \\
\hline NbS00020788g0012 & $N b 20788$ & & AtPBL 13, AtRIPK & Lin et al., 2015; Liu et al., 2011 \\
\hline NbS00000608g0110 & Nb608 & Nb11711 & NtACIK 1 & Rowland et al., 2005 \\
\hline NbS00011711g0016 & Nb11711 & Nb608 & NtACIK1 & Rowland et al., 2005 \\
\hline NbS00058880g0009 & Nb58880 & & SLACIK1 & Rowland et al., 2005 \\
\hline NbS00033699g0007 & Nb33699 & & AtCST & Burr et al., 2011 \\
\hline NbS00009691g0004 & Nb9691 & Nb29137 & AtNAK & $\begin{array}{l}\text { Moran \& Walker, 1993; } \\
\text { Kodama et al., } 2009\end{array}$ \\
\hline NbS00029137g0012 & Nb29137 & Nb9691 & AtNAK & $\begin{array}{l}\text { Moran \& Walker, 1993; } \\
\text { Kodama et al., } 2009\end{array}$ \\
\hline NbS00024800g0006 & $N b 24800$ & & AtNAK & $\begin{array}{l}\text { Moran \& Walker, 1993; } \\
\text { Kodama et al., } 2009\end{array}$ \\
\hline NbS00042854g0003 & $N b 42854$ & & $A t B I K 1 / A t P B L 1$ & $\begin{array}{l}\text { Veronese et al., 2006; } \\
\text { Zhang et al., } 2010\end{array}$ \\
\hline NbS00001051g0011 & Nb1051 & & $\begin{array}{l}\text { SITPK } 1 b / A t A P K 1 a, \\
\text { AtAPK } 1 b\end{array}$ & $\begin{array}{l}\text { AbuQumar et al., 2008; } \\
\text { Hirayama \& Oka, } 1992\end{array}$ \\
\hline NbS00045149g0002 & Nb45149 & Nb16559 & & \\
\hline NbS00016559g0013 & Nb16559 & & & \\
\hline NbS00001529g0015 & Nb1529 & & & \\
\hline NbS00028219g0012 & Nb28219 & & $A t P B L 2 ; A t A P K 2 b$ & Ito et al., 1997; Zhang et al., 2010 \\
\hline NbS00029131g0004 & Nb29131 & Nb7877 & AtPBL2; AtAPK $2 b$ & Ito et al., 1997; Zhang et al., 2010 \\
\hline NbS00007877g0013 & Nb7877 & Nb29131 & AtPBL2; AtAPK $2 b$ & Ito et al., 1997; Zhang et al., 2010 \\
\hline
\end{tabular}

Table S2. PCR primers employed for cloning eGFP-tagged constructs of putative $\mathbf{N}$. benthamiana and tomato BIK1 homologues. Gateway sites for directional cloning are underlined.

\begin{tabular}{|l|l|l|}
\hline Primer code & Primer name * & Sequence \\
\hline ao81 & Nb42854-cacc-fw & caccATGGGGTCTTGTATCAGTGTTC \\
\hline ao82 & Nb42854-rv-nostop & AGTAACAAGCTGAGGAGAGGCA \\
\hline ao83 & SI11520-cacc-fw & cacCATGGGTTCTTGTTTAAGTGTTCG \\
\hline ao84 & SI11520-rv & TGTAACAAGGGGAGAAGCAGC \\
\hline
\end{tabular}

${ }^{*} \mathrm{fw}$, forward; rv, reverse 
Table S3. PCR primers employed for cloning VIGS fragments of putative $\mathbf{N}$. benthamiana BIK1 homologues. Introduced restriction sites (Xbal at the $5^{\prime}$-end, and Acc65I-Spel double restriction site at the $3^{\prime}$-end) for directional cloning are underlined.

\begin{tabular}{|c|c|c|}
\hline Primer code & Primer name * & Sequence \\
\hline THO1 & FwNb42854 & tctagaACCAGCCATTCTTTGTGACC \\
\hline THO2 & RvNb42854 & ggtaccactagtCTTCCGTCCGGATAGTGTGT \\
\hline $\mathrm{THO} 3$ & FwNb9691 & tctagaCTACCGAGTCAAGCAGGATATTAGA \\
\hline $\mathrm{THO} 4$ & RvNb9691 & ggtaccactagtAATCTCTTTCCTATCTGCCAGTTTT \\
\hline THO5 & FwNb1051 & tctagaGGGTATTGCTTGGAGGATGA \\
\hline THO6 & RvNb1051 & ggtaccactagtCAGCTCCAAGAGCAACCTTC \\
\hline THO7 & FwNb28219 & tctagaCCAGGTTTGGATGACGAAGT \\
\hline THO8 & RvNb28219 & ggtaccactagtAGCCAGAGGGTTTTCAAGGT \\
\hline THO9 & FwNb29137 & tctagaTCAAATCAGCAGTCGCAAAC \\
\hline THO10 & RvNb29137 & ggtaccactagtGAAAATAGCCCTTGGTGCTG \\
\hline THO11 & FwNb24800 & tctagaGGAGAGCATGGAGACAGACC \\
\hline THO12 & RvNb24800 & ggtaccactagtAAGCAAGGATGGTGTCGAAG \\
\hline THO13 & FwNb58880 & tctagaAACCTCCTCAGACCCCACTT \\
\hline THO14 & RvNb58880 & ggtaccactagtTCATGGGGAAGAAAAACCAG \\
\hline THO15 & FwNb20788 & tctagaAACTGGTCCAAACCCTCCTT \\
\hline THO16 & RvNb20788 & ggtaccactagtTTCCATGGGATTCCAGTCTC \\
\hline THO17 & FwNb7877 & tctagaCGTCGATGCAATAACCAATG \\
\hline THO18 & RvNb7877 & ggtaccactagtAATTGAAGCCGGAAGGTTTT \\
\hline THO19 & FwNb29131 & tctagaAGGTCACAAGGAGTGGTTGG \\
\hline THO20 & RvNb29131 & ggtaccactagtACACCAAGAGGTGGTTGTCC \\
\hline THO23 & FwNb1529 & tctagaCCATAAGGAGTGGCTGGTGT \\
\hline THO24 & RvNb1529 & ggtaccactagtTCACCTTCCAAGCAGAATCC \\
\hline THO27 & FwNb16559 & tctagaTCCTTCAATTGCATGAACCA \\
\hline THO28 & RvNb16559 & ggtaccactagtTAACTCCAATGCGGAGAACC \\
\hline THO29 & FwNb45149 & tctagaCATATCCAAACCCТCССТCA \\
\hline THO30 & RvNb45149 & ggtaccactagtCCCTGGCAGTGAGAGAAAAG \\
\hline THO31 & FwNb33699 & tctagaTCTGGGTACTCGCCAATTTC \\
\hline THO32 & RvNb33966 & ggtaccactagtTGGGACATGCAGAAAATCAA \\
\hline THO33 & FwNb11711 & tctagaCCTTCTGGACCATCTTTTTGC \\
\hline THO34 & RvNb11711 & ggtaccactagtTTGCCGGTGAAACTAAATGA \\
\hline THO35 & FwNb608 & tctagaGGCACCCACTACTCAATCGT \\
\hline THO36 & RvNb608 & ggtaccactagtAAGATGGCCCAGAAGGAGAT \\
\hline
\end{tabular}

${ }^{*} \mathrm{FW}$, forward; Rv, reverse 
Table S4. PCR primers employed for cloning split-luciferase constructs of putative tomato BIK1 homologues. Introduced restriction sites for directional cloning are underlined.

\begin{tabular}{|c|c|c|}
\hline Primer code & Primer name * & Sequence \\
\hline JRO01 & Kpnl-Solyc08g061250-fwd & CGGGGTACCATGACTGGTGAGAGATTAAGCTG \\
\hline JRO02 & Sall-Solyc08g061250-rev & ACGCGTCGACATGTCTTCGATAGAGAGCTGTATC \\
\hline JRO03 & Kpnl-Solyc07g041940.2-fwd & CGGGGTACCATGAAGATTGGATGGGAATC \\
\hline JRO04 & Sall-Solyc07g041940.2-rev & ACGCGTCGACGGATCTCTTGAAACCATTTTG \\
\hline JRO05 & Kpnl-Solyc04g082500.2-fwd & CGGGGTACCATGGGAAATTGTTGGCC \\
\hline JRO06 & Sall-Solyc04g082500.2-rev & ACGCGTCGACATAGCTACGGTTTTTTGGAGC \\
\hline JRO07 & Kpnl-Solyc11g062400.1-fwd & CGGGGTACCATGGGGATTTGCTTTGG \\
\hline JRO08 & Sall-Solyc11g062400.1-rev & ACGCGTCGACTCTTGAATGTGTCACATATCTCTT \\
\hline JRO09 & Kpnl-Solyc06g062920.2-fwd & CGGGGTACCATGGCTACTTGCGGAATTG \\
\hline JRO10 & Sall-Solyc06g062920.2-rev & ACGCGTCGACATAAGAATTTAGTTTGTTTGTTCTTTC \\
\hline JRO11 & Kpnl-Solyc05g025820.2-fwd & CGGGGTACCATGAAGATTACATGGGAATCTCTAG \\
\hline JRO12 & Sall-Solyc05g025820.2-rev & ACGCGTCGACAGCTCTATGGAAATCATTGTGC \\
\hline JRO13 & Kpnl-Solyc01g088690.2-fwd & CGGGGTACCATGAAGTGTTTTTTTTAATTCAAG \\
\hline JRO14 & Xhol-Solyc01g088690.2-rev & CCGCTCGAGTGTAGTTTTACCCTTCTGCATG \\
\hline JRO15 & Kpnl-Solyc05g007050.2-fwd & CGGGGTACCATGGGATTAGGTGGTGATG \\
\hline JRO16 & Sall-Solyc05g007050.2-rev & ACGCGTCGACAGTTTTGCCGTTTGGTTT \\
\hline JRO17 & Kpnl-Solyc08g077560.2-fwd & CGGGGTACCATGCTGAAGTGTTTTTATATATTCA \\
\hline JRO27 & Sall-Solyc08g077560.2-rev & ACGCGTCGACAGCCTGAGCTAAGG \\
\hline $\mathrm{JRO} 21$ & Kpnl-Solyc01g112220.2-fwd & CGGGGTACCATGGGAGTTTGTTTCAGTTCTAA \\
\hline $\mathrm{JRO} 22$ & Sall-Solyc01g112220.2-rev & ACGCGTCGACTGATGCTCTTTTTGGGAGTG \\
\hline $\mathrm{JRO} 23$ & Kpnl-Solyc06g005500.2-fwd & CGGGGTACCATGGGGATATGTTTGAGTGC \\
\hline $\mathrm{JRO} 24$ & Sall-Solyc06g005500.2-rev & ACGCGTCGACTTTAGCGTAAAGGGGAGAAG \\
\hline Lo94 & SISOBIR1-Kpn1-fw & CGGCGCGGTACCATGACTTCGAATATCCACTTTTTTCTTTTATACG \\
\hline Lo95 & SISOBIR1-Sal1-NoStop-rv & GCCGCCGTCGACATGCTTGATCTGAGTTAACATGC \\
\hline Lo153 & GUS-Kpn1-FW & CGGCGGGGTACCATGTTACGTCCTGTAGAAACC \\
\hline Lo154 & GUS-Sal1-RV & GAAGCCGTCGACTTGTTTGCCTCCCTGCTGCGGTTTTTC \\
\hline
\end{tabular}

${ }^{*} \mathrm{fwd} / \mathrm{fw} / \mathrm{FW}$, forward; rev/rv/RV, reverse 



\section{Chapter 6}

\section{Studies on BIR1 and its possible role as a negative regulator of Cf-4/SOBIR1-mediated immunity in Solanaceous plants}

Aranka M. van der Burgh'1, Sergio A. Landeo-Villanueva', Jim Renema ${ }^{1}$, Michael F. Seidl ${ }^{1}$, Matthieu H. A. J. Joosten ${ }^{1}$ 


\section{Abstract}

Cell surface receptors of the plant innate immune system enable the plant to sense extracellular signals upon attack by pathogens. These transmembrane receptors, which can be receptor-like kinases (RLKs) or receptor-like proteins (RLPs), need to be tightly controlled and regulated to ensure the triggering of a robust defence signal, and to prevent false activation of defence responses. The BAK1-Interacting RLK (BIR) protein family of Arabidopsis thaliana is a group of RLKs with four members, BIR1 to BIR4, which negatively regulate immunity by interfering with the heterodimerization of the co-receptor BRI1-Associated Kinase 1/Somatic Embryogenesis Receptor Kinase 3 (BAK1/SERK3) with ligand-binding immune receptors. Here we show that the BIR protein family is conserved in Solanaceous plants. As the expression patterns of BIR 1 and $B I R 2$, in Arabidopsis plants treated with a diverse set of pathogens and pathogen elicitors, is similar to the expression patterns of SOBIR 1 and BAK1/SERK3, whereas the expression patterns of $B I R 3$ and $B I R 4$ is not, we focus our studies on BIR1 and BIR2. By performing gene silencing, overexpression, and protein-protein interaction studies, we show that the tomato BIR1 orthologue interacts with BAK1/SERK3 and is a negative regulator cell death, and that BIR1 might suppress the Avr4-triggered hypersensitive response $(\mathrm{HR})$ in tomato containing $\mathrm{Cf}-4$. Additionally, we show that a BIR2 orthologue of tomato also interacts with BAK1/SERK3, but Solanaceous BIR2 orthologs seem not to be involved in modulating the Cf-4/Avr4-triggered HR. This suggests that BIR1 and BIR2 each likely interact with a different pool of BAK1/ SERK3 present at the plasma membrane, and that each pool is probably involved in a different defence pathway. 


\section{Introduction}

Plants do not have an adaptive immune system like mammals. Instead, their innate immune system consists of hundreds of cell surface- and cytoplasmic receptors, which enable the plant to sense extracellular and intracellular signals upon attack by pathogens (Dodds \& Rathjen, 2010). Transmembrane (TM)-receptors that monitor the extracellular space can be either receptor-like kinases (RLKs) or receptor-like proteins (RLPs) (Macho \& Zipfel, 2014). RLKs have an extracellular domain (often consisting of leucine-rich repeats, LRRs), a single TM domain, and an intracellular kinase domain. LRR-RLPs have a structure similar to LRR-RLKs (further referred to as RLPs and RLKs), but lack an intracellular kinase domain, and instead form bimolecular RLKs by constitutively interacting with the RLK Suppressor Of BIR1-1/Evershed (SOBIR1/EVR, further referred to as SOBIR1) (Liebrand et al., 2013 and 2014; Gust \& Felix, 2014).

Well known examples of RLKs are Flagellin-Sensing 2 (FLS2) and the EF-Tu Receptor (EFR), sensing the flg22 peptide from bacterial flagellin and the elf18 peptide from bacterial Elongation Factor-Tu, respectively (Gómez-Gómez et al., 2000; Zipfel et al., 2006). Recognition of flg22 or elf18 leads to basal defence responses, including callose deposition and reactive oxygen species (ROS) production (Gómez-Gómez et al., 2000; Zipfel et al., 2006). Examples of well-studied RLPs are RLP23 and Cf-4. RLP23 from Arabidopsis thaliana (further referred to as Arabidopsis) recognizes the NLP20 peptide derived from the Necrosis and Ethylene-inducing Peptide 1 (NEP1)Like Protein, which is a widely conserved microbial pattern (Böhm et al., 2014). Recognition of NLP20 leads to basal defence responses similar to the responses that are triggered upon recognition of flg22 or elf18 (Albert et al., 2015). Cf-4 is an RLP from tomato, which confers resistance to Cladosporium fulvum upon recognition of the fungal apoplastic effector Avr4 (Thomas et al., 1997). Recognition of Avr4 leads to strong defence responses visible as HR (Joosten et al., 1994; Thomas et al., 1997). Along with many other RLPs, both RLP23 and Cf-4 have been shown to constitutively interact with the regulatory RLK SOBIR1 to form a bimolecular RLK (Liebrand et al., 2013; Bi et al., 2014; Gust \& Felix; 2014).

Specific activation of immunity often includes the hetero- and/or homodimerization of receptors into receptor complexes (Macho \& Zipfel, 2014; Han et al., 2014). Indeed, upon pathogen recognition, RLKs like FLS2 and EFR, and bimolecular RLKs such as RLP23/SOBIR1 and Cf-4/SOBIR1, recruit the co-receptor BRI1-Associated Kinase 1/ Somatic Embryogenesis Receptor Kinase 3 (BAK1/SERK3, further referred to as BAK1) to initiate downstream signalling, leading to defence activation (Heese et al., 2007; Chinchilla et al., 2007; Albert et al., 2015; Postma et al., 2016).

All receptor complexes mentioned above are involved in the initiation of defence responses, which are highly energy-consuming (Belkhadir et al., 2014; Lozano-Durán 
\& Zipfel., 2015). Consequently, these receptors need to be tightly controlled and regulated, on the one hand to ensure the triggering of a quick and robust signal, but on the other hand also to ensure a fast relief of the activated state after signalling (Couto \& Zipfel, 2016; Cao, 2016). Furthermore, the activation of defence responses eventually may lead to a hypersensitive response (HR), which is a kind of programmed cell death (Lam et al., 2001; Greenberg \& Yao, 2004). Failure to accurately regulate immunity, including appropriate negative regulation by switching on the breaks in time, may lead to the constitutive activation of immune responses observed as developmental defects, like dwarfing and even spontaneous systemic cell death (Gao et al., 2009; Lorrain et al., 2013). The regulation of immune receptor activation is secured at several levels. For instance, the phosphorylation state of immune receptors is tightly controlled by kinases and phosphatases, and the formation of higher order receptor complexes is negatively regulated by (pseudo-)kinases (Couto \& Zipfel, 2016; Segonzac et al., 2014; Couto et al., 2016).-

The family of BAK1-Interacting RLK (BIR) proteins from the model plant Arabidopsis has been shown to be a group of (pseudo-)kinases that negatively regulate BAK1mediated immunity (Gao et al., 2009; Halter et al., 2014a and 2014b; Imkampe et al., 2017). There are four BIR homologues in Arabidopsis: AtBIR1 to AtBIR4, and all four of them are able to interact with AtBAK1 at the plasma membrane (PM) (Gao et al., 2009; Halter et al., 2014b; Ma et al., 2017; Hohmann et al., 2018). All AtBIRs are RLKs with an extracellular LRR-domain, a TM-domain, and an intracellular kinase-domain, but actually only the kinase domain of AtBIR1 is an active kinase, whereas the others are pseudokinases (Gao et al., 2009; Halter et al., 2014b).

AtBIR1 was initially found as a negative regulator of immunity and cell death, as bir1 knockout plants show seedling lethality and strong upregulation of defence responses, including enhanced salicylic acid (SA) synthesis and enhanced expression of various pathogenesis-related (PR) genes (Gao et al., 2009). Negative regulation by AtBIR1 is partially suppressed by AtSOBIR1, as a knockout of this RLK in Arabidopsis attenuates the bir1 phenotype, hence its name Suppressor Of BIR1-1. Additionally, BIR 1 silencing in Arabidopsis increases the pool of SOBIR1 proteins interacting with BAK1 (Liu et al., 2016). BIR1 does not seem to affect flg22-induced ROS and mitogenactivated protein kinase (MAPK) $3 / 6$ activation, as knocking out bir 1 in Arabidopsis has no effect on flg22-mediated growth inhibition (Gao et al., 2009) and overexpressing BIR1 in Arabidopsis has no effect on the flg22-triggered ROS burst or flg22-triggered MAPK3/6 activation (Liu et al., 2016). MAPK4 activation upon flg22 treatment does seem to be compromised in bir 1 plants, and this effect is diminished under elevated temperature conditions (Gao et al., 2009).

The pseudokinase AtBIR2 has no enzymatic activity, so it cannot transautophosphorylate or transphosphorylate another protein, but it is transphosphorylated itself by AtBAK1 (Blaum et al., 2014; Halter et al., 2014a and 2014b). AtBIR2 interacts 
with AtBAK1 in the resting state, whereby AtBIR2 sequesters AtBAK1 and prevents it from forming active immune complexes with ligand-binding receptors (Halter et al., 2014b). Upon ligand recognition by RLKs like AtFLS2 and AtEFR, AtBAK1 is released from AtBIR2, allowing AtBAK1 to associate with the ligand-bound receptor and to initiate immune signalling by trans-phosphorylation events between the kinase domains of AtBAK1 and AtFLS2 (Halter et al., 2014b). By keeping AtBAK1 away from ligand-binding receptors in the resting state, AtBIR2 negatively regulates immune signalling. Accordingly, BIR2 knockout Arabidopsis plants show enhanced levels of SA and enhanced expression of $P R$ genes, which are typical features of defence activation (Halter et al., 2014b). Additionally, BIR2 knockout Arabidopsis plants show increased resistance to biotrophic pathogens, such as the bacterial pathogen Pseudomonas syringae pv tomato DC3000 (Halter et al., 2014b). On the other hand, a BIR2 knockout leads to increased susceptibility to the necrotrophic fungus Alternaria brassicicola, a pathogen that benefits from cell death upon plant colonisation. This indicates that cell death plays a role in the type of immunity that is suppressed by AtBIR2 (Halter et al., 2014b). However, cell death does not seem to be as severely induced in BIR2knockouts as compared to BIR1-knockout plants (Gao et al., 2009; Halter et al., 2014b). This observation suggests that BIR1 and BIR2 might at least partially exert their suppressive effect in different defence signalling pathways.

Recently, the pseudokinase AtBIR3 has been found to not only interact with the co-receptor BAK1, but also with several ligand-binding receptors like FLS2, EFR, and Brassinosteroid-Insensitive 1 (BRI1) (Imkampe et al., 2017; Hohmann et al., 2018). BRI1 plays a role in development by dimerizing with BAK1 upon recognition of the brassinosteroid (BR) hormone (Nam \& Li, 2002). AtBIR3 suppresses the ligand-induced association of BAK1 with BRI1, FLS2, and EFR, and thereby negatively regulates immunity and BR signalling (Imkampe et al., 2017; Hohmann et al., 2018). Additionally, the BIR3 ortholog from tomato, SIBIR3, has also been shown to suppress BAK1 receptor complex formation and thereby BAK1-mediated immunity and BR-signalling in tomato (Huang et al., 2017).

The BIR family appears to be conserved in the Solanaceous plants Nicotiana benthamiana and tomato (Saur et al., 2016; Huang et al., 2017). The expression patterns of BIR1 and BIR2, in Arabidopsis plants treated with a diverse set of pathogens and pathogen elicitors, is similar to the expression patterns of SOBIR 1 and BAK1/SERK3, whereas the expression patterns of $B I R 3$ and $B I R 4$ is not, so we focus our studies on BIR1 and BIR2. We aim to analyse the role of BIR1 and BIR2 in the Cf-4/SOBIR1-mediated $\mathrm{HR}$ upon recognition of Avr4. We show that the orthologues of BIR1 in N. benthamiana and tomato are negative regulators of cell-death, and that BIR1 appears to suppress the Avr4-triggered HR in tomato containing Cf-4. Additionally, we show that NbBIR2 and SIBIR2 are not involved in modulating the Cf-4/Avr4-triggered HR. This suggests that BIR1 and BIR2 each likely interact with a different pool of BAK1 present at the 
plasma membrane, and that each pool might be involved in a different defence pathway leading to either a strong defence response (HR), for instance triggered by Avr4, or a weak basal defence response, for instance triggered by flg22.

\section{Results \& Discussion}

\section{Arabidopsis expression data reveal that BIR1 and BIR2 potentially play a role in SOBIR1-mediated immunity}

There are four homologous BIR proteins in Arabidopsis, and AtBIR1 clusters separately from AtBIR2, AtBIR3 and AtBIR4 (Fig. 1A). The BIR protein family is conserved throughout the Plant Kingdom (Fig. S1), and both N. benthamiana and tomato contain BIR homologues (Saur et al., 2016; Huang et al., 2017). Tomato has a single BIR1homologue, referred to as SIBIR1, and two BIR2-homologues, indicated as SIBIR2A and SIBIR2B (Fig. 1A). N. benthamiana has two homologues for each, named NbBIR1A and B, and NbBIR2A and B (Saur et al., 2016). AtBIR3 and AtBIR4 seem to have evolved by duplication in Brassicaceae after speciation from Solanaceae, as we find one tomato orthologue for BIR3/4, named SIBIR3/4 (Huang et al., 2017), and three orthologues in $N$. benthamiana for BIR3/4, named NbBIR3/4A, B, and C (Fig. 1A).

Co-regulation of gene expression is commonly observed for genes that play a role in the same pathway or process (Lee et al., 2015; Rhee \& Mutwil, 2014). To study possible co-regulation of the expression of the various BIRs with SOBIR 1 and BAK1, we mined publicly available data that show regulation of gene expression in Arabidopsis upon treatment with several pathogens and elicitors (Toufighi et al., 2005). From the overview provided in Fig. 1B, it is clear that the pattern of gene expression of BIR1 and $B I R 2$ positively correlates with the pattern of gene expression of BAK1 and SOBIR 1 upon pathogen and elicitor treatment, as their rate of upregulation is similar (Fig. 1B and Table S1). This positive correlation of expression of SOBIR1 and BAK1 seems even more apparent for $B I R 1$ than for BIR2. Co-regulation of the expression of $B I R 1$ and $B I R 2$ with $B A K 1$ and SOBIR 1 hints to a regulatory role of the encoded BIR1 and BIR2 proteins for the RLKs SOBIR1 and BAK1. In contrast, the expression patterns of BIR3 and BIR4 show no clear correlation with BAK1 and SOBIR 1 upon pathogen treatment, and BIR4 even shows a negative correlation for Arabidopsis inoculated with the obligate biotroph Hyaloperonospora arabidopsidis (Fig. 1B and Table S1).

Recent publications show a role in immunity not only for BIR1 and BIR2 (Gao et al., 2009; Halter et al., 2014a and 2014b), but also for BIR3 (Huang et al., 2017; Imkampe et al., 2017). The negative correlation of BIR4 expression could also hint to a regulatory role of BIR4 for the SOBIR1 and BAK1 RLKs. However, we here chose to focus on the role of BIR1 and BIR2 in Cf-4/SOBIR1/BAK1-mediated immunity, based on our phylogenetic analyses and on the clear co-regulation of their expression with BAK1 and SOBIR1. 


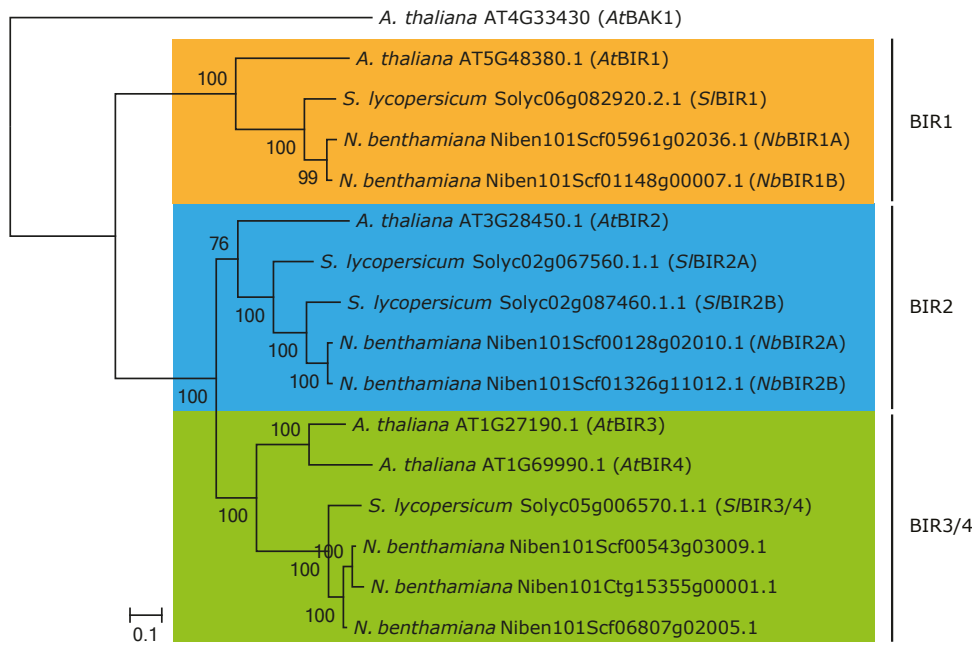

B

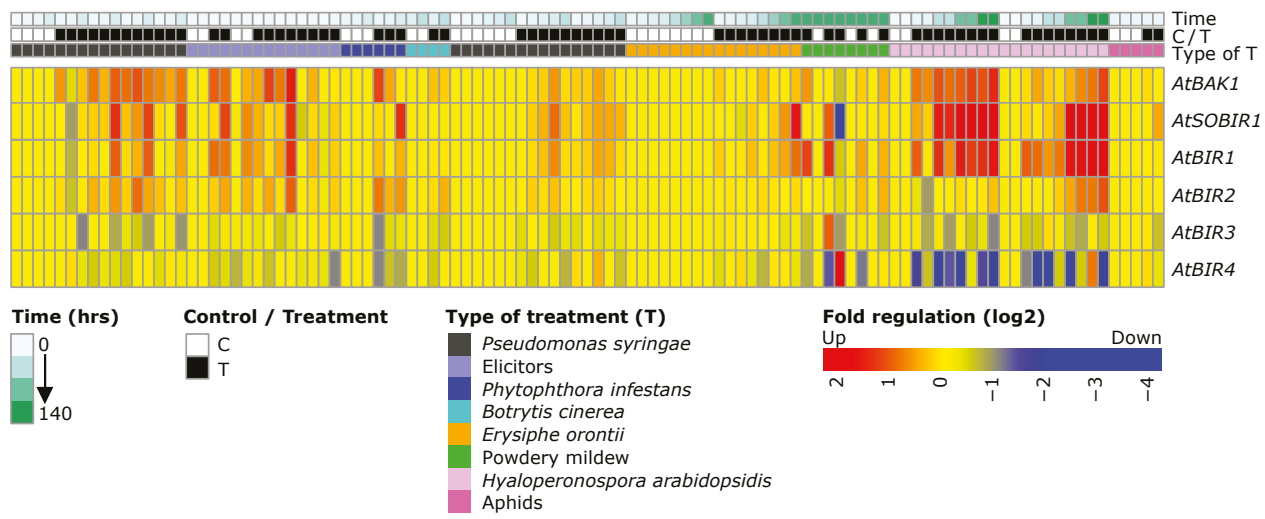

Figure 1. The BIR protein family is conserved in Arabidopsis, N. benthamiana and tomato, and there is a positive correlation between the expression of BIR1, BIR2, SOBIR1 and BAK1 in Arabidopsis under various biotic stresses. (A) Phylogenetic tree of protein sequences of AtBIR1 (orange), AtBIR2 (blue), and AtBIR3/4 (green), and their homologues in N. benthamiana and tomato. Bootstrap values are indicated. The branch length represents the genetic change. AtBAK1 is used as an out-group. See also Fig. S1. (B) Heat map of gene expression in Arabidopsis upon pathogen or elicitor treatments shows that the expression profiles of $A t B I R 1$ and $A t B I R 2$ positively correlate with the expression profiles of AtSOBIR1 and $A t B A K 1$. The expression profiles of $A t B I R 3$ and AtBIR4 do not correlate, or even show negative correlation for certain treatments. The latter is most apparent for AtBIR4. Arabidopsis expression data have been retrieved from online available data (Toufighi et al., 2005). See also Table S1. 


\section{BIR1 from Solanaceous plants is a negative regulator of cell death and might be involved in Cf-4/SOBIR1-mediated immunity, whereas BIR2 does not appear to play a role in Cf-4/SOBIR1-mediated immunity}

To elucidate whether BIR1 and BIR2 play a role in Cf-4/SOBIR1-mediated immunity, we silenced the expression of either the BIR1 or BIR2 orthologues in $N$. benthamiana:Cf-4 to assess their possible involvement in the Avr4-induced $H R$. For this, we used pTRV2:BIR 1 and pTRV2:BIR2, which target both the $A$ and $B$ homologues of $N b B I R 1$ and $N b B I R 2$, respectively. Virus-induced gene silencing (VIGS) of BIRTAB in N. benthamiana:Cf-4 plants resulted in a lethal phenotype (Fig. 2A). This phenotype is similar to the phenotype observed in Arabidopsis, as knocking out BIR1 in Arabidopsis leads to systemic cell death and very small plants (Gao et al., 2009). This observation confirms that we have identified the functional orthologues of AtBIR1 in N. benthamiana. Silencing of BIR2AB in $N$. benthamiana:Cf-4 did not result in an apparent phenotype, although the $B I R 2 A B$ gene knockdown was found to be successful (Fig. 2B). No clear phenotype was anticipated to occur, as a knockout of BIR2 in Arabidopsis is also not lethal (Halter et al., 2014b).

As BIR1-silencing was lethal, we could only test BIR2 for its possible involvement in the Cf-4/Avr4-mediated HR. For this, we agro-infiltrated Avr4, or Avr9 as negative control, into leaves of the BIR2-silenced $N$. benthamiana:Cf-4 plants. We did not observe an effect on the Cf-4/Avr4-triggered HR (Fig. 2A and 2C), so BIR2 is probably not involved in Cf-4/SOBIR1-mediated immunity. Targeting Cf-4 by VIGS confirmed that knockdown of Cf-4 suppresses the Avr4-induced HR (Gabriëls et al., 2006) (Fig. 2A and 2C).

To analyse the role of the BIR1 and BIR2 homologues of tomato in the Cf-4/Avr4triggered HR by VIGS, we used the same constructs as were used to perform VIGS in $N$. benthamiana, as pTRV2:BIR1 also targets SIBIR1, and pTRV2:BIR2 also targets SIBIR2B and likely SIBIR2A (Fig. S2). Inoculation of MM-Cf-4 tomato with pTRV2:BIR1 did not lead to plant death, but did result in a dwarf phenotype (Fig. 2D), probably because silencing in tomato is less efficient than in N. benthamiana (Liu et al., 2002a; Gabriëls, 2006). The dwarfing phenotype confirmed that we have identified the functional orthologues of AtBIR1 in tomato. Inoculation of MM-Cf-4 tomato with pTRV2:BIR2 did not result in any symptoms, similar to VIGS in N. benthamiana:Cf-4 using this construct, and similar to knocking out BIR2 in Arabidopsis (Halter et al., 2014b). 

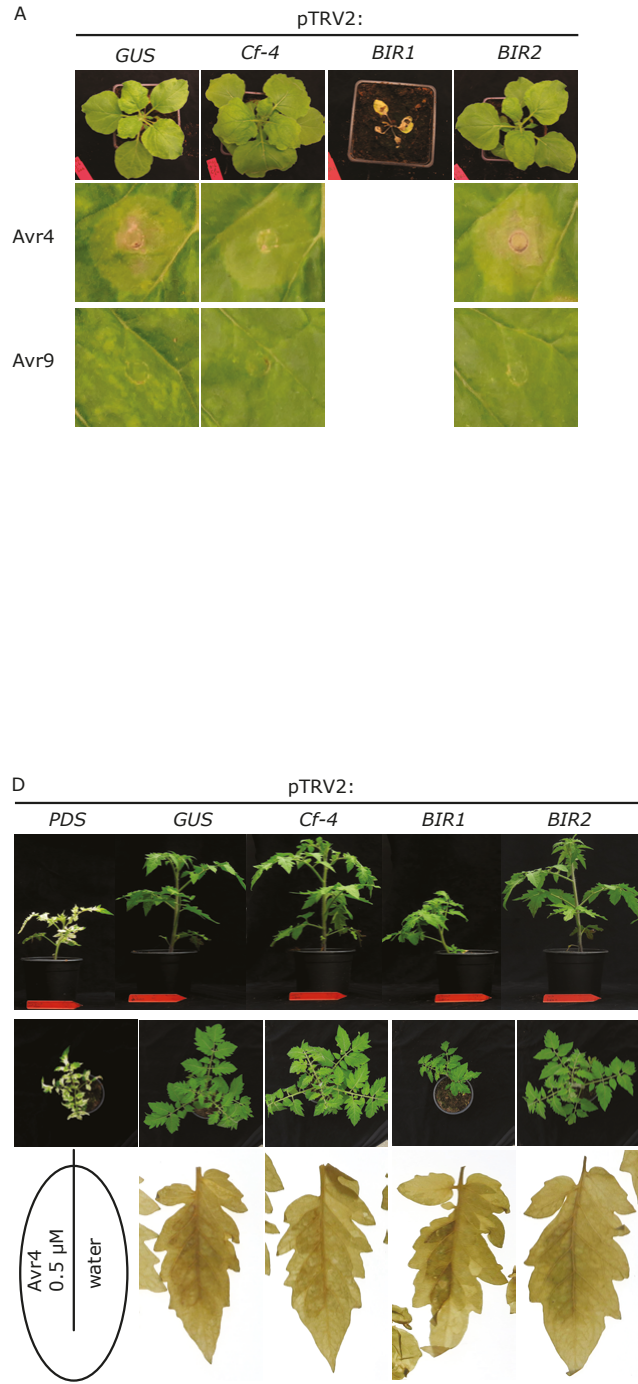
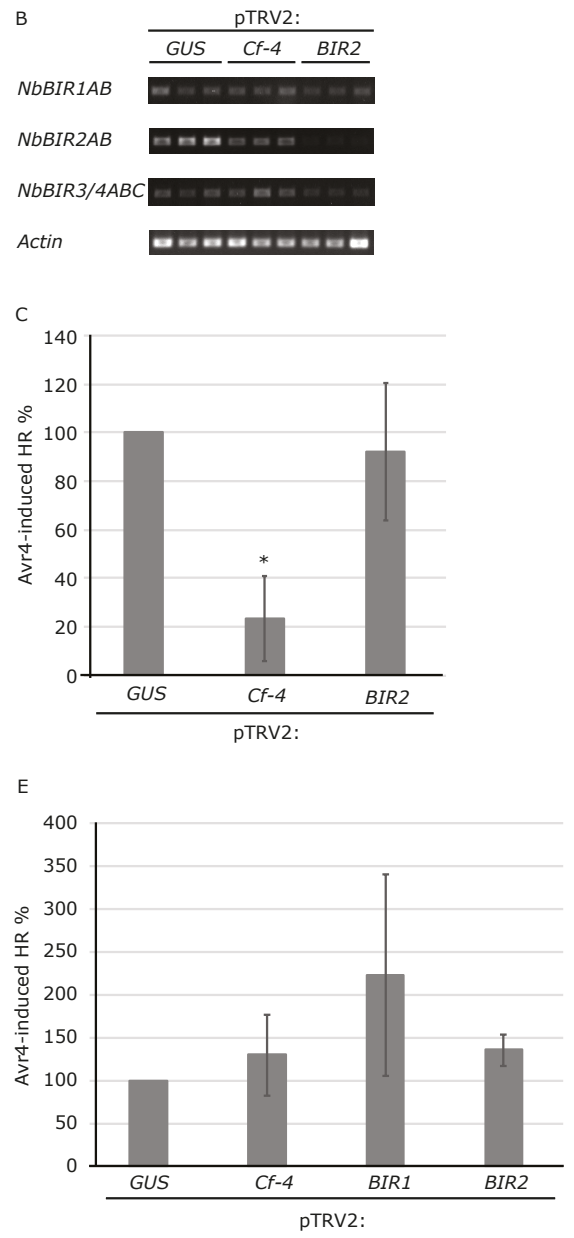

Figure 2. BIR1 from Solanaceous plants is a negative regulator of cell death and might be involved in Cf-4/SOBIR1-mediated immunity, whereas BIR2 does not appear to play a role in Cf-4/SOBIR1mediated immunity. (A) Silencing of $N b B I R 1 A B$ in $N$. benthamiana is lethal, and silencing of $N b B I R 2 A B$ has no visible effect. Pictures of $N$. benthamiana:Cf- 4 plants were taken at 3 weeks after inoculation with the indicated TRV recombinants, which was the time-point at which the leaves were agroinfiltrated with either Avr4 or Avr9 at an $\mathrm{OD}_{600}$ of 0.03 . Pictures of the infiltrated leaf areas were taken at 3 days post infiltration (dpi). The experiment was performed three times, with at least four plants per experiment, of which two leaves per plant were infiltrated. Representative pictures are shown. (B) The expression of $N b B I R 2 A B$ is specifically downregulated upon inoculation of $N$. benthamiana with pTRV2:BIR2. The expression of $N b B I R 1 A B$, NbBIR3/4, and NbActin is not affected by inoculation with pTRV2:GUS, pTRV2:Cf-4, or pTRV2:BIR2. A 30 cycle RT-PCR was performed on cDNA obtained from $N$. benthamiana:Cf-4 plants, three weeks after inoculation with the various TRV recombinants. (C) Quantification of the data shown in panel A. Silencing of Cf-4 in $N$. benthamiana:Cf-4 leads to a significantly reduced Avr4-induced HR, whereas silencing of NbBIR2AB does 
not. The HR was scored as described in the Materials \& Methods section. Data are represented as mean +/- standard error (SE), and the intensity of the HR observed in GUS-silenced plants was set at $100 \%$. The presence of a significant difference as compared to the intensity of the HR in GUS-silenced plants was determined using a student's t-test $(p<0.05)$. (D) Inoculation of MM-Cf-4 tomato with pTRV2:BIR1 leads to a dwarf phenotype, whereas inoculation with pTRV2:BIR2 has no visible effect. Pictures of the plants were taken 3 weeks after inoculation with the indicated TRV recombinants. At that time point, leaves were infiltrated with $0.5 \mu \mathrm{M}$ Avr4 or water as a negative control. At $1 \mathrm{dpi}$, leaves were harvested and destained with ethanol to visualize the areas where cell death had occurred. Note that tomato inoculated with PTRV2:BIR1 seems to show a slightly stronger Avr4-induced HR. As at 1 dpi the HR only just starts to appear, a reduced HR in pTRV2:Cf-4-inoculated plants can only be observed at 2 dpi (not shown). The experiment was performed three times, including at least three plants per experiment and infiltration of four leaflets per plant. Representative pictures are shown. (E) Inoculation of tomato with pTRV2:SICf-4 or pTRV2:BIR2 does not lead to an altered Avr4-induced HR at $1 \mathrm{dpi}$. Inoculation with pTRV2:BIR1 seems to lead to a slightly increased Avr4-induced HR, although this increase is proven not to be significant. The HR was scored as described in the Materials \& Methods section. Data are represented as mean $+/-\mathrm{SE}$, and the intensity of the HR observed in pTRV2:GUS-inoculated plants was set at 100\%. No significant difference between the various treatments and the pTRV2:GUS-inoculated control plants was obtained using a student's t-test.

As the silencing in tomato is probably only partial, this allowed us to also screen the pTRV2:BIR1-inoculated MM-Cf-4 plants, next to the pTRV2:BIR2-inoculated MM-Cf-4 plants, for a possible effect on the Avr4-induced HR. Infiltration of MM$\mathrm{Cf}-4$ tomato with a $0.5 \mu \mathrm{M}$ solution of Avr4 protein results in a clear HR after two days (Joosten et al., 1994). We checked whether an HR would occur already after one day, to monitor a possible faster HR response upon BIR1 or BIR2AB silencing. Additionally, we harvested leaves one day after Avr4-infiltration and de-stained them with ethanol to visualize possible early cell death (Fig. 2D). Interestingly, in plants previously inoculated with PTRV2:BIR1, the HR started slightly earlier, although this could not be significantly quantified (Fig. 2E). The pTRV2:BIR2-inoculated plants did not show an altered timing of the Avr4-induced HR. Additional analysis of the BIR1silenced tomato leaves to determine possible $P R$ gene upregulation, in addition to changes in defence-related hormone levels, should shed more light on possible negative regulation of immunity by BIR1 in tomato. Furthermore, the knockdown of SIBIR1, SIBIR2A, and SIBIR2B should be confirmed by RT-PCR.

The VIGS experiments in N. benthamiana:Cf-4 and tomato presented here, show that also in these Solanaceous plants BIR1 is a negative regulator of cell death. Together with the correlation of gene expression of especially BIR1 with SOBIR1 and BAK1, the VIGS experiments indicate a possible role as negative regulator for BIR1 in Cf-4/SOBIR1-mediated immunity.

\section{BIR1 and BIR2 overexpression in N. benthamiana:Cf-4 does not affect the Cf-4/ Avr4-triggered HR}

As silencing of $B I R T A B$ in $N$. benthamiana resulted in a lethal phenotype, we were not able to examine its role in the Cf-4/Avr4-triggered HR in such an assay (Fig. 
2A). Therefore, we set out to test the contrary, and overexpressed the different BIRs to determine whether this would suppress the $\mathrm{Cf}-4 /$ Avr4-triggered HR. To test this hypothesis, we overexpressed BIR1 and BIR2 from Arabidopsis (Halter et al., 2014b) and their homologues from tomato in N. benthamiana:Cf-4, together with Avr4, or Avr9 as a negative control (Fig. 3A). AtBIR1 and AtBIR2 have previously been shown to properly accumulate upon their overexpression (Halter et al., 2014b), and in the experiment described below, we show that also SIBIR1 and SIBIR2B properly accumulate upon their overexpression (Fig. 4). Unfortunately, we were not able to clone SIBIR2A from tomato CDNA. Remarkably, overexpression of the different BIRs did not reveal an effect on the Cf-4/Avr4-triggered HR when compared to the GUS overexpression control. A slight decrease of the intensity of the HR appears to take place for the co-infiltration of Avr4 with AtBIR1 and also with SIBIR1 (Fig. 3A), although this effect proved not to be significant (Fig. 3B).

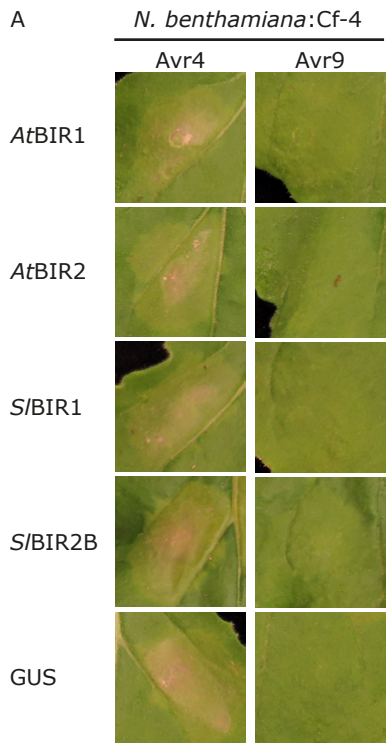

B

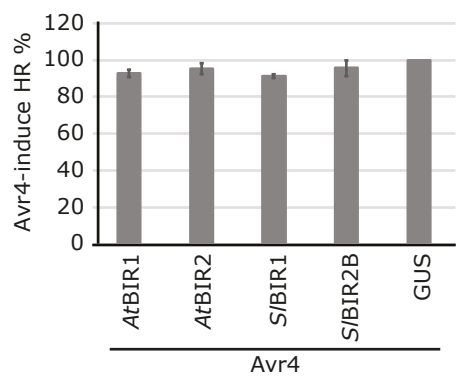

Figure 3. BIR1 and BIR2 overexpression in $N$. benthamiana:Cf-4 does not affect the Cf-4/Avr4triggered HR. (A) YFP-tagged AtBIR1 and AtBIR2, and eGFP-tagged SIBIR1 and SIBIR2B were transiently coexpressed in $N$. benthamiana:Cf-4 in combination with silencing suppressor $\mathrm{P} 19$, at an $\mathrm{OD}_{600}$ of 1 for each construct, and Avr4 or Avr9 were included at an $\mathrm{OD}_{600}$ of 0.03. Co-expression of GUS-eGFP was used as a control. At 3 dpi the HR was scored. Experiments were repeated four times, with infiltration of at least four leaves per repetition. (B) Quantification of the Cf-4/Avr4-triggered $H R$, as observed in panel A. The HR obtained upon coagro infiltration of GUS and Avr4 was set at $100 \%$. The HR was scored as described in the Materials \& Methods section. There was no significant difference in the levels of the $H R$ at $p<0.05$, as determined by one-way ANOVA, including a Tukey post hoc-test. 
We hypothesised that the increased BIR1 protein pool, upon the overexpression of Arabidopsis or tomato BIR1, would sequester increased amounts of endogenous NbBAK1 in the resting state, thereby leading to a reduced availability of BAK1 to form active receptor complexes upon activation of the Cf-4/SOBIR1 complex by its matching ligand, Avr4. Consequently, we expected that overexpression of BIR1 would negatively regulate immunity, to be visualized as a decrease in HR. However, we did not observe an effect in the Avr4-triggered HR that was tested here. In the experiment employed here, the constructs driving BIR- and Avr4-expression were simultaneously delivered, and thereby these proteins are probably more or less accumulating simultaneously. Therefore, Avr4 might already have activated the Cf-4/SOBIR1/BAK1 immune-complex before a sufficient amount of BIR1 protein has accumulated to prevent the sequestering of BAK1 from such activating complexes. Additionally, the affinity of BAK1 for the activated Cf-4/SOBIR1 complex might be higher than for the overexpressed BIRs (Hohmann et al., 2018). Future experiments, in which Avr4 is delivered after BIR proteins have sufficiently accumulated, might shed more light on the possible role of BIR1, and perhaps also BIR2, in the regulation of the activity of Cf-4/SOBIR1-containing immune complexes.

\section{SIBIR1 and SIBIR2B interact with SIBAK1 in planta}

To study whether the tomato BIR and BAK1 proteins interact in planta, we transiently overexpressed SIBIR1 and SIBIR2B with SIBAK1 in N. benthamiana:Cf-4. Co-IP experiments reveal that SIBIR1 and SIBIR2B both interact with SIBAK1 in planta (Fig. 4). This was expected, as NbBIR1A, NbBIR2A, NbBIR2B, and all four AtBIR proteins have earlier been shown to interact with NbBAK1 and AtBAK1, respectively (Saur et al., 2016; Gao et al., 2009; Halter et al., 2014b; Imkampe et al., 2017).

We hypothesize that BAK1 is dissociated from BIR1 upon Avr4 elicitation, after which BAK1 is recruited to the Cf-4/SOBIR1 bimolecular RLK. We do not expect BAK1 dissociation from BIR2 upon Avr4 treatment, as silencing of BIR2 did not affect the Avr4-triggered HR (Fig. 2). As a positive control we tested the dissociation of BAK1 from BIR2 upon flg22 elicitation, after which released BAK1 then activates the RLK FLS2 (Halter et al., 2014b). To test our hypothesis, we transiently overexpressed SIBIR1 and SIBIR2B with SIBAK1 in N. benthamiana:Cf-4 (which contains the native flg22 receptor NbFLS2 (Hann \& Rathjen, 2007)), and then elicited the plant with Avr4 or flg22. By subsequent co-IP experiments, we were not able to show a release of BAK1 from SIBIR1 upon Avr4 treatment, or a release of BAK1 from SIBIR2 upon flg22 treatment (Fig. 4).

AtBIR2 and AtBIR3 have been shown before to be dissociated from BAK1 upon treatment of Arabidopsis with the flg22 peptide that is perceived by FLS2 (Halter et al., 2014b; Imkampe et al., 2017). However, this dissociation was tested in Arabidopsis plants for endogenous AtBAK1 using antiserum detecting the protein itself, in co- 
IPs with endogenous AtBIR2 or stably expressing AtBIR3 fused to a GFP tag (Halter et al., 2014b; Imkampe et al., 2017). Probably, the lack of an apparent BIR-BAK1 dissociation in our assay might be because we employed transient overexpression. By overexpression, the various proteins strongly accumulate, and minor differences in the amounts of co-immunoprecipitating BAK1 protein with the BIR proteins upon elicitor treatment, might not be distinguishable. Furthermore, only a small pool of $\mathrm{BIR}$ and BAK1 proteins might be involved in the regulation of FLS2 and Cf-4/SOBIR1 responses, and overexpression of these proteins probably causes an excess of the various proteins as a result of which we cannot observe any difference in this small pool.
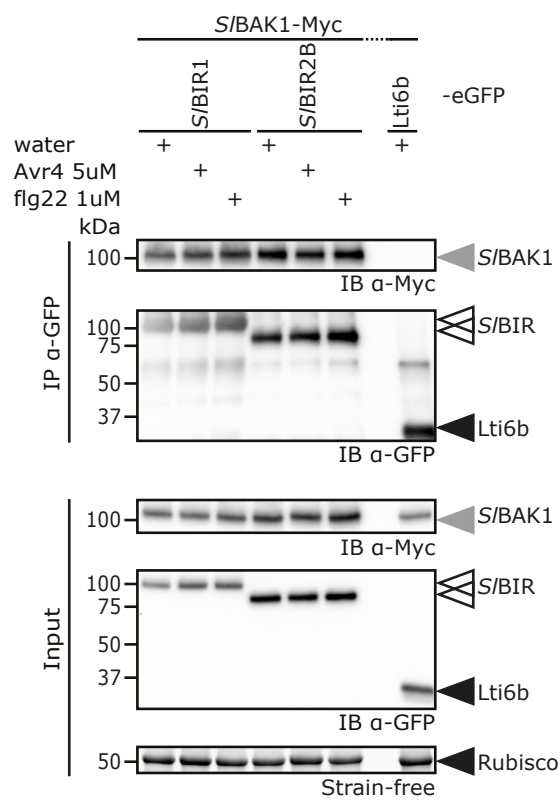

Figure 4. SIBIR1 and SIBIR2B interact with SIBAK1 in planta, but ligand-dependent dissociation cannot be shown in transient expression assays. SIBIR1-eGFP and SIBIR2B-eGFP were transiently expressed in combination with S/BAK1-Myc and P19 in $N$. benthamiana:Cf-4, at an $\mathrm{OD}_{600}$ of 0.6 for each construct. The small TM protein Low TemperatureInduced 6b (LTI6b) (Kurup et al., 2005) was included as a negative control (lane 8). Lane 7 is empty. At 2 dpi, leaves were infiltrated with Avr4, flg22, or water, and harvested after 15 minutes, followed by an IP using GFP affinity beads, and protein detection on IB. Note that SIBAK1-Myc co-immunoprecipitates with both SIBIR1 and SIBIR2B in all treatments. The Rubisco band in the input samples indicates equal loading. The experiment was repeated twice and representative blots are shown.

Additionally, the release of BIR2 and BIR3 from BAK1 in Arabidopsis was not very apparent upon flg22 elicitation alone, but elicitation with a cocktail of elicitors did enhance the dissociation of BAK1 from BIR2 and BIR3 (Halter et al., 2014b; Imkampe et al., 2017). Future experiments to test for a possible dissociation of SIBAK1 from SIBIR1, and perhaps from S/BIR2A and/or SIBIR2B, upon activation of RLP/SOBIR1 bimolecular RLKs, should include elicitor cocktails consisting of elicitors that trigger the activation of several HR-inducing RLP/SOBIR1 complexes simultaneously. This will help to further unravel the possible function of BIR1, and possibly BIR2, in RLP/ SOBIR1 signalling for immunity.

In conclusion, the co-IP experiments support the interaction of SIBAK1 with SIBIR1 and S/BIR2B, but a possible dissociation of BIR/BAK1 complexes upon elicitor treatment could not be shown. 


\section{BIR1, in contrast to BIR2, might negatively regulate the Cf-4/SOBIR1 immune response}

Heterodimerization of receptor pairs is a well-known step in the activation of immune signalling (Macho \& Zipfel, 2014; Han et al., 2014; Hohmann et al., 2017). However, how different signals are passed on upon dimerization of different primary ligand receptors with the same co-receptor, BAK1, is not yet clear.

Specific differential phosphorylation of the kinase domains of the interacting receptor and the co-receptor BAK1 has been proposed to be a key to differential downstream signalling (Wang et al., 2008; Schwessinger et al., 2011; Hohmann et al., 2017). For instance threonine residue T450 of AtBAK1 is essential for the flg22triggered response, but not for plant development, as AtBAK1 $1^{\mathrm{T} 450 \mathrm{~A}}$ can rescue the seedling-lethal phenotype of bak1-1/bak1-like(bkk)1-1 Arabidopsis plants, but cannot complement for the flg22 insensitivity (Wang et al., 2008). This suggests that differential phosphorylation of $A t B A K 1^{T 450}$ is important to initiate different downstream responses (Wang et al., 2008).

BIR1 carries an active cytoplasmic kinase domain, although no 'RD' motif is present, and this active kinase domain is essential for the role of BIR1 as a negative regulator of defence responses (Gao et al., 2009). The kinase activity of BIR1 apparently contributes to the ability to suppress strong constitutive immune-responses, as a BIR1 knockout in Arabidopsis is lethal, whereas a BIR2 knockout is not (Gao et al., 2009; Halter et al., 2014b). Furthermore, BIR1-silencing in N. benthamiana is also lethal, but BIR2-silencing not (Fig. 2). In contrast to BIR1, the RLKs BIR2, BIR3, and probably also BIR4 all carry a kinase domain that lacks kinase activity (pseudokinase) (Halter et al., 2014b; Imkampe et al., 2017) and therefore these RLKs might regulate immune responses that are less strong.

Another way to achieve differential signalling upon recruitment of the same coreceptor can be via the formation of nanoclusters, in which specific sets of receptors, co-receptors, and downstream involved proteins reside (Bücherl et al., 2017). Indeed, several research lines have resulted in the discovery that different TM-receptors are not homogeneously distributed over the PM, but remain separated in different nanoclusters (Jarsch et al., 2014; Hutten at al., 2017; Bücherl et al., 2017). Different outputs of signalling by BAK1 in a heterodimer with FLS2 or with BRI1 might therefore be explained by the occurrence of different pools of BAK1 in distinct nanoclusters, in combination with either FLS2 or BRI1 and their associated downstream signalling partners (Bücherl et al., 2017). Whether the presence of specific receptor/co-receptor combinations in distinct nanoclusters is a cause or a consequence of differential phosphorylation of the cytoplasmic kinase domains of the different RLKs remains to be elucidated. 
We hypothesise that BIR1 and BIR2 are present in different nanoclusters, to negatively regulate immune signalling by BAK1 in combination with different primary ligand receptors. We propose a model (Fig. 5) in which the pool of BAK1 that is required to trigger basal immune responses by TM-receptors such as FLS2, EFR, and the RLP23/SOBIR1 complex, is present in a complex with BIR2, whereas another pool of BAK1, that plays a role in strong defence responses (leading to $H R$ ) triggered by TM-receptors such as the Cf-4/SOBIR1 complex, is negatively regulated by BIR1.

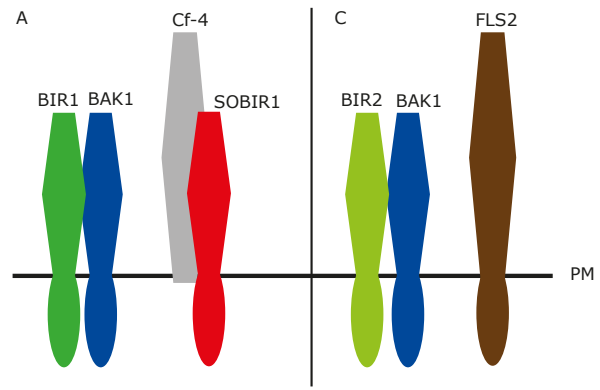

Cf-4/SOBIR1 in resting state FLS2 in resting state

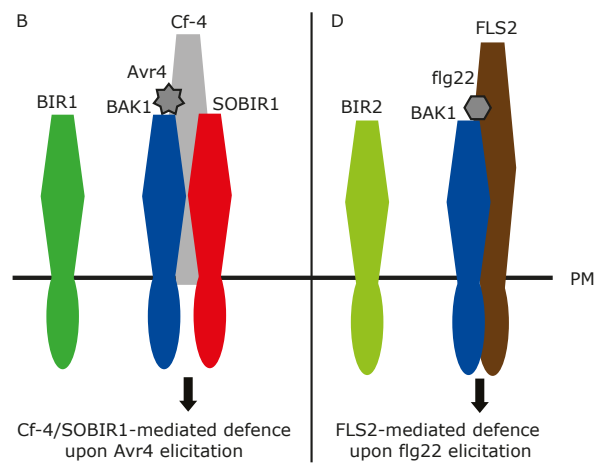

Figure 5. BIR1, in contrast to BIR2, might negatively regulate the Cf-4/SOBIR1 immune response. In this model we propose that BIR1 (dark green), in contrast to BIR2 (light green), negatively regulates the activation of Cf-4/SOBIR1 (grey and red, respectively) immune complexes, by sequestering BAK1 (blue) (A). In the resting state, BAK1 is kept away from forming active signalling complexes with the Cf-4/SOBIR1 complex by BIR1 (A). Upon Avr4 elicitation, BAK1 dissociates from BIR1, and a strong defence response is activated by the Cf-4/SOBIR1/BAK1 complex, eventually resulting in an HR (B). In the resting state, BAK1 is kept away from forming active signalling complexes with TM-receptors like FLS2 that trigger basal defence responses, by BIR2 (C). Upon flg22 elicitation, BAK1 is released from BIR2, and a basal defence response is activated by the FLS2/BAK1 complex (D). PM, plasma membrane. 


\section{Conclusions \& Future prospects}

Accurate regulation of the activity of immune receptors is essential for plant homeostasis and for an adequate response to invading pathogens. Therefore, this regulation takes place at multiple levels. The BIR family of Arabidopsis is a group of RLKs that negatively regulate immunity by interfering with the heterodimerization of the co-receptor BAK1 with ligand-binding immune receptors (Halter et al., 2014a and 2014b; Imkampe et al., 2017). Blocking this heterodimerization prevents transphosphorylation events to take place between the kinase domains of BAK 1 and the primary immune receptor, thereby negatively regulating defence activation. The study presented here at least partially sheds light on the role of BIR1 and BIR2 in the negative regulation of immune signalling in $N$. benthamiana and tomato. We identified functional orthologs of Arabidopsis BIR1 and BIR2 in N. benthamiana and tomato and reveal that BIR1, in contrast to BIR2, likely negatively regulates the $C f-4$ / SOBIR1 immune response in Solanaceous plants. Further research is necessary to clarify the exact role of $B I R 1$, and possibly $B I R 2$, in the negative regulation of $C f-4 /$ SOBIR1 and other RLP/SOBIR1 immune complexes.

\section{Materials \& Methods}

\section{Phylogenetic and expression analysis of the BIR family}

Homologous BIR protein sequences from various plant species, including tomato and $N$. benthamiana, were retrieved from phytozome (phytozome.jgi.doe.gov/pz/ portal.html) and www.solgenomics.net, and aligned using the Mafft web-server (Stamatakis et al., 2008). An unrooted maximum likelihood tree was built using phylogeny.fr (Dereeper et al., 2008 and 2010), using G-blocks to eliminate poorly aligned positions and divergent regions, and viewed using MEGA5.

The expression analysis of AtBAK 1, AtSOBIR 1, and AtBIR 1, 2, 3 and 4 was performed by consulting the e-Northerns w. Expression Browser (bar.utoronto.ca; Toufighi et al., 2005). The Atgen express - pathogen series was selected, and the average of replicate treatments relative to the average of the appropriate control treatments was analysed for the indicated genes.

\section{Binary vectors for Agrobacterium-mediated transformation and virus-induced gene silencing}

The binary vector expressing the silencing suppressor P19 (Voinnet et al., 2015), and plasmids pTRV1, pTRV2:PDS (Liu et al., 2002a and 2002b), pTRV2:GUS (Tameling \& Baulcombe, 2007), and pTRV2:Cf-4 (Gabriëls et al., 2006), have been described elsewhere. The construct pBIN-KS-35S::SISERK3-myc has been described previously 
(Mantelin et al., 2011). Avr4 and Avr9 were expressed using the pMOG800-based constructs (Van der Hoorn et al., 2000). The construction of pBIN-KS-35S::GUSeGFP (SOL 5094) is described in chapter 3 of this thesis. The plasmids PB7YWG235S-AtBIR1-YFP and PB7YWG2-35S-AtBIR2-YFP were obtained from Halter et al. (2014b), and transformed to Agrobacterium tumefaciens strain C58C1, carrying the pCH32 helper plasmid. The construct expressing the GFP-tagged TM protein Low Temperature-Induced 6b (GFP-LTI6b) has been described previously (Kurup et al., 2005).

To generate pTRV2:NbBIRTAB (SOL 7120) and pTRV2:NbBIR2AB (SOL 7121) (further referred to as PTRV2:BIR1 and PTRV2:BIR2), virus-induced gene silencing (VIGS) constructs that target the $N$. benthamiana homologues of BIR1 or BIR2, respectively, polymerase chain reactions (PCRs) were performed on $N$. benthamiana CDNA, using the primers listed in Table S2. PCR products were restriction-digested and ligated into pTRV2 (pYL156; Liu et al., 2002b), using Xbal and Ncol restriction enzymes.

To overexpress the tomato $B I R$ genes, $S I B I R 1$ and $S I B I R 2 B$ coding regions were amplified from tomato cDNA using the primers listed in Table S2. Despite several attempts, we were not able to amplify SIBIR2A. SIBIR1 and SIBIR2B were cloned into pENTR/D-Topo, and a subsequent LR reaction with Gateway LR Clonase enzyme mix II (Invitrogen) and pBIN-KS-35S::GWY-eGFP (Sol 2095, for C-terminal tagging with eGFP) resulted in the binary vectors pBIN-KS-35S::SIBIR1-eGFP (SOL 7105) and pBINKS-35S::SIBIR2B-eGFP (SOL 7106). For transient plant transformation, the vectors were introduced in $A$. tumefaciens strain $\mathrm{C} 58 \mathrm{C} 1$, carrying the $\mathrm{pCH} 32$ helper plasmid.

\section{Plant growth conditions}

$N$. benthamiana stably expressing SICf-4 under control of its native promoter (referred to as N. benthamiana:Cf-4) (Gabriëls et al., 2006), was grown in the climate chamber under $16 \mathrm{~h}$ of light at $25^{\circ} \mathrm{C}$ and $8 \mathrm{~h}$ of darkness at $21^{\circ} \mathrm{C}$, at $\sim 75 \%$ relative humidity. Tomato MM-Cf-4 (Thomas et al., 1997) was grown in the greenhouse at 16 $\mathrm{h}$ of light at $21^{\circ} \mathrm{C}$ and $8 \mathrm{~h}$ of darkness at $19^{\circ} \mathrm{C}$, at $\sim 75 \%$ relative humidity.

\section{VIGS in N. benthamiana and tomato}

VIGS using TRV-based vectors was performed in N. benthamiana:Cf-4 and tomato MM-Cf-4 as described previously (Liebrand et al., 2012). To verify silencing in $N$. benthamiana:Cf-4, RNA was isolated from $N$. benthamiana:Cf-4 leaves, three weeks after inoculation with the various TRV recombinants, using the Quick RNA miniprep kit (Zymoclean). Primers for reverse transcriptase (RT-)PCR were designed to amplify both $N b B I R 1$ homologues, both NbBIR2 homologues, or the three $N b B I R 3 / 4$ homologues (Table S2). 


\section{Agrobacterium-mediated transient transformation}

Agrobacterium-mediated transient transformations of $N$. benthamiana:Cf-4 were performed as previously described (Van der Hoorn et al., 2000). Avr4 and Avr9 were agro-infiltrated at an $\mathrm{OD}_{600}$ of 0.03 . The occurrence and intensity of an HR was determined at 3 days post infiltration (dpi), unless indicated otherwise. A five-way score for visual HR was used: $130 \%$ for a faster $H R, 100 \%$ for $H R, 60 \%$ for suppressed $H R, 30 \%$ for strongly suppressed $H R$, and $0 \%$ for no HR. Suppressed $H R$ was observed as a slower and less intense response.

To perform co-immunoprecipitation (co-IP) assays between the different S/BIR homologues and SIBAK1, transient transformations of $N$. benthamiana:Cf-4 were performed at an $\mathrm{OD}_{600}$ of 0.6 per construct, with co-expression of P19 also at an $\mathrm{OD}_{600}$ of 0.6. After 2 days, leaves were infiltrated with Avr4 protein $(0.5 \mu \mathrm{M})$, flg22 peptide $(1 \mu \mathrm{M} ;$ Genscript), or water, and leaves were harvested after 15 minutes.

\section{Analysis of Avr4-triggered HR in tomato}

Three weeks after the inoculation of ten-day-old tomato MM-Cf-4 seedlings with TRV recombinants, leaves were infiltrated with $0.5 \mu \mathrm{M}$ Avr4 protein or water. After one day, leaves were harvested and de-stained with ethanol to visualize the leaf areas that had mounted an HR. The five-way score for HR was used, as described above.

\section{Co-IPs between tomato BAK1 and BIR proteins}

Co-IPs between tomato BAK1 and BIR proteins were performed as described previously (Liebrand et al., 2013). Pre-cast TGX stain-free gels were used for protein analyses (Bio-Rad \#456-8085), and total protein was visualized using the stainfree method. The following antibodies were used for protein detection on western blots: $\alpha$-GFP-HRP (130-091-833, MACS), $\alpha$-Myc (cMyc9E10, sc-40, Santa Cruz), and $\alpha-$ Mouse-HRP (GE healthcare).

\section{Acknowledgements}

We thank Unifarm personnel for excellent plant care. We acknowledge Hanna Rovenich for providing Avr4 protein. A.M.v.d.B. is supported by the Netherlands Organization for Scientific Research (NWO), Earth and Life Sciences (ALW). 


\section{References}

Albert I, Böhm H, Albert M, Feiler CE, Imkampe J, Wallmeroth N, Brancato C, Raaymakers TM, Oome S, Zhang H, Krol E, Grefen C, Gust AA, Chai J, Hedrich R, Van den Ackerveken G, Nürnberger T. 2015. An RLP23-SOBIR1-BAK1 complex mediates NLP-triggered immunity. Nature Plants 1: doi:10.1038/nplants.2015.1140.

Belkhadir Y, Yang L, Hetzel J, Dangl JL, Chory J. 2014. The growth-defense pivot: crisis management in plants mediated by LRR-RK surface receptors. Trends in Biochemical Sciences 39: 447-456.

Bi G, Liebrand TW, Cordewener JH, America AH, Xu X, Joosten MHAJ. 2014. Arabidopsis thaliana receptor-like protein AtRLP23 associates with the receptor-like kinase AtSOBIR1. Plant Signaling \& Behavior 9: e27937.

Blaum BS, Mazzotta S, Nöldeke ER, Halter T, Madlung J, Kemmerling B, Stehle T. 2014. Structure of the pseudokinase domain of BIR2, a regulator of BAK1-mediated immune signaling in Arabidopsis. Journal of Structural Biology 186: 112-121.

Böhm H, Albert I, Oome S, Raaymakers TM, Van den Ackerveken G, Nürnberger T. 2014. A conserved peptide pattern from a widespread microbial virulence factor triggers pattern-induced immunity in Arabidopsis. PLOS Pathogens 10: e1004491.

Bücherl CA, Jarsch IK, Schudoma C, Segonzac C, Mbengue M, Robatzek S, MacLean D, Ott T, Zipfel C. 2017. Plant immune and growth receptors share common signalling components but localise to distinct plasma membrane nanodomains. elife 6: e25114.

Cao X. 2016. Self-regulation and cross-regulation of pattern-recognition receptor signalling in health and disease. Nature Reviews Immunology 16: 35-50.

Chinchilla D, Zipfel C, Robatzek S, Kemmerling B, Nürnberger T, Jones JD, Felix G, Boller T. 2007. A flagellin-induced complex of the receptor FLS2 and BAK1 initiates plant defence. Nature 448: 497-500.

Couto D, Niebergall R, Liang X, Bücherl CA, Sklenar J, Macho AP, Ntoukakis V, Derbyshire P, Altenbach D, Maclean D, Robatzek S, Uhrig J, Menke F, Zhou J-M, Zipfel C. 2016. The Arabidopsis protein phosphatase PP2C38 negatively regulates the central immune kinase BIK1. PLOS Pathogens 12: e1005811.

Couto D, Zipfel C. 2016. Regulation of pattern recognition receptor signalling in plants. Nature Reviews Immunology 16: 537-552.

Dereeper A, Blanc G, Claverie J-M, Audic S. 2010. BLAST-EXPLORER helps you building datasets for phylogenetic analysis. BMC evolutionary biology 10: 8 .

Dereeper A, Guignon V, Blanc G, Audic S, Buffet S, Chevenet F, Dufayard J-F, Guindon S, Lefort V, Lescot M. 2008. Phylogeny. fr: robust phylogenetic analysis for the non-specialist. Nucleic Acids Research 36: W465-W469.

Dodds PN, Rathjen JP. 2010. Plant immunity: towards an integrated view of plant-pathogen interactions. Nature Reviews Genetics 11: 539-548.

Domínguez-Ferreras A, Kiss-Papp M, Jehle A, Kristina, Felix G, Chinchilla D. 2015. An overdose of the Arabidopsis coreceptor BAK1 or its ectodomain causes autoimmunity in a SOBIR1-dependent manner. Plant Physiology 168: 1106-1121.

Gabriëls SHEJ, Takken FLW, Vossen JH, De Jong CF, Liu Q, Turk SCHJ, Wachowski LK, Peters J, Witsenboer HMA, De Wit PJGM, Joosten MHAJ. 2006. CDNA-AFLP combined with functional analysis reveals novel genes involved in the hypersensitive response. Molecular Plant-Microbe Interactions 19: 567-576.

Gabriëls SHEJ. 2006. Functional analysis of tomato genes expressed during the Cf-4/Avr4-induced hypersensitive response. PhD thesis, Wageningen University. Chapter 3: p63-86

Gao M, Wang X, Wang D, Xu F, Ding X, Zhang Z, Bi D, Cheng YT, Chen S, Li X, Zhang Y. 2009. Regulation of cell death and innate immunity by two receptor-like kinases in Arabidopsis. Cell Host and Microbe 6: 34-44.

Gómez-Gómez L, Boller T. 2000. FLS2: an LRR receptor-like kinase involved in the perception of the bacterial elicitor flagellin in Arabidopsis. Molecular Cell 5: 1003-1011.

Greenberg JT, Yao N. 2004. The role and regulation of programmed cell death in plant-pathogen interactions. Cellular Microbiology 6: 201-211. 
Gust AA, Felix G. 2014. Receptor like proteins associate with SOBIR1-type of adaptors to form bimolecular receptor kinases. Current Opinion in Plant Biology 21: 104-111.

Halter T, Imkampe J, Blaum BS, Stehle T, Kemmerling B. 2014a. BIR2 affects complex formation of BAK1 with ligand binding receptors in plant defense. Plant Signaling \& Behavior 9: 134-143.

Halter T, Imkampe J, Mazzotta S, Wierzba M, Postel S, Bücherl C, Kiefer C, StahI M, Chinchilla D, Wang X, Nürnberger T, Zipfel C, Clouse S, Borst Jan W, Boeren S, de Vries Sacco C, Tax F, Kemmerling B. 2014b. The Leucine-Rich Repeat Receptor Kinase BIR2 Is a Negative Regulator of BAK1 in Plant Immunity. Current Biology 24: 134-143.

Han Z, Sun Y, Chai J. 2014. Structural insight into the activation of plant receptor kinases. Current Opinion in Plant Biology 20: 55-63.

Hann DR, Rathjen JP. 2007. Early events in the pathogenicity of Pseudomonas syringae on Nicotiana benthamiana. The Plant Journal 49: 607-618.

Heese A, Hann DR, Gimenez-Ibanez S, Jones AME, He K, Li J, Schroeder JI, Peck SC, Rathjen JP. 2007. The receptor-like kinase SERK3/BAK1 is a central regulator of innate immunity in plants. Proceedings of the National Academy of Sciences of the United States of America 104: 12217-12222.

Hohmann U, Lau K, Hothorn M. 2017. The structural basis of ligand perception and signal activation by receptor kinases. Annual Review of Plant Biology 68: 109-137.

Hohmann U, Nicolet J, Moretti A, Hothorn LA, Hothorn M. 2018. The SERK3 elongated allele defines a role for BIR ectodomains in brassinosteroid signalling. Nature Plants. 10.1038/s41477-018-0150-9

Huang S, Nie S, Wang S, Liu J, Zhang Y, Wang X. 2017. SIBIR3 Negatively Regulates PAMP Responses and Cell Death in Tomato. International Journal of Molecular Sciences 18: 1966.

Hutten SJ, Hamers DS, Den Toorn MA, Van Esse W, Nolles A, Bücherl CA, De Vries SC, Hohlbein J, Borst JW. 2017. Visualization of BRI1 and SERK3/BAK1 Nanoclusters in Arabidopsis Roots. PLoS ONE 12: e0169905.

Imkampe J, Halter T, Huang S, Schulze S, Mazzotta S, Schmidt N, Manstretta R, Postel S, Wierzba M, Yang Y, vanDongen WM, Stahl M, Zipfel C, Goshe MB, Clouse S, de Vries SC, Tax F, Wang X, Kemmerling B. 2017. The Arabidopsis leucine-rich repeat receptor kinase BIR3 negatively regulates BAK1 receptor complex formation and stabilizes BAK1. The Plant Cell 29: 2285-2303.

Jarsch IK, Konrad SSA, Stratil TF, Urbanus SL, Szymanski W, Braun P, Braun K-H, Ott T. 2014. Plasma membranes are subcompartmentalized into a plethora of coexisting and diverse microdomains in Arabidopsis and Nicotiana benthamiana. The Plant Cell 26: 1698-1711.

Joosten MHAJ, Cozijnsen TJ, De Wit PJ. 1994. Host resistance to a fungal tomato pathogen lost by a single base-pair change in an avirulence gene. Nature 367: 384.

Kurup S, Runions J, Köhler U, Laplaze L, Hodge S, Haseloff J. 2005. Marking cell lineages in living tissues. The Plant Journal 42: 444-453.

Lam E, Kato N, Lawton M. 2001. Programmed cell death, mitochondria and the plant hypersensitive response. Nature 411: 848-853.

Lee T, Kim H, Lee I. 2015. Network-assisted crop systems genetics: network inference and integrative analysis. Current Opinion in Plant Biology 24: 61-70.

Liebrand TWH, Smit P, Abd-El-Haliem A, de Jonge R, Cordewener JHG, America AHP, Sklenar J, Jones AME, Robatzek S, Thomma BPHJ, Tameling WIL, Joosten MHAJ. 2012. Endoplasmic reticulumquality control chaperones facilitate the biogenesis of Cf receptor-like proteins involved in pathogen resistance of tomato. Plant Physiology 159: 1819-1833.

Liebrand TWH, van den Berg GCM, Zhang Z, Smit P, Cordewener JHG, America AHP, Sklenar J, Jones AME, Tameling WIL, Robatzek S, Thomma BPHJ, Joosten MHAJ. 2013. Receptor-like kinase SOBIR1/ EVR interacts with receptor-like proteins in plant immunity against fungal infection. Proceedings of the National Academy of Sciences of the United States of America 110: 10010-10015.

Liebrand TWH, van den Burg HA, Joosten MHAJ. 2014. Two for all: receptor-associated kinases SOBIR1 and BAK1. Trends in Plant Science 19: 123-132.

Liu Y, Huang X, Li M, He P, Zhang YC. 2016. Loss-of-function of Arabidopsis receptor-like kinase BIR1 activates cell death and defense responses mediated by BAK1 and SOBIR1. New Phytologist 212: 637645. 
Liu Y, Schiff M, Dinesh-Kumar S. 2002a. Virus-induced gene silencing in tomato. The Plant Journal 31: 777-786.

Liu Y, Schiff M, Marathe R, Dinesh-Kumar S. 2002b. Tobacco Rar1, EDS1 and NPR1/NIM1 like genes are required for $\mathrm{N}$-mediated resistance to tobacco mosaic virus. The Plant Journal 30: 415-429.

Lorrain S, Vailleau F, Balagué C, Roby D. 2003. Lesion mimic mutants: keys for deciphering cell death and defense pathways in plants? Trends in Plant Science 8: 263-271.

Lozano-Durán R, Zipfel C. 2015. Trade-off between growth and immunity: role of brassinosteroids. Trends in Plant Science 20: 12-19.

Ma C, Liu Y, Bai B, Han Z, Tang J, Zhang H, Yaghmaiean H, Zhang Y, Chai J. 2017. Structural basis for BIR1mediated negative regulation of plant immunity. Cell Research 27: 1521.

Macho AP, Zipfel C. 2014. Plant PRRs and the activation of innate immune signaling. Molecular Cell 54: 263-272.

Mantelin S, Peng HC, Li B, Atamian HS, Takken FL, Kaloshian I. 2011. The receptor-like kinase SISERK1 is required for Mi-1-mediated resistance to potato aphids in tomato. The Plant Journal 67: 459-471.

Nam KH, Li J. 2002. BRI1/BAK1, a receptor kinase pair mediating brassinosteroid signaling. Cell 110: 203-212.

Postma J, Liebrand TWH, Bi G, Evrard A, Bye RR, Mbengue M, Kuhn H, Joosten MHAJ, Robatzek SC. 2016. Avr4 promotes Cf-4 receptor-like protein association with the BAK1/SERK3 receptor-like kinase to initiate receptor endocytosis and plant immunity. New Phytologist 210: 627-642.

Rhee SY, Mutwil M. 2014. Towards revealing the functions of all genes in plants. Trends in Plant Science 19: 212-221.

Saur IM, Kadota Y, Sklenar J, Holton NJ, Smakowska E, Belkhadir Y, Zipfel C, Rathjen JP. 2016. NbCSPR underlies age-dependent immune responses to bacterial cold shock protein in Nicotiana benthamiana. Proceedings of the National Academy of Sciences of the United States of America 113: 3389-3394.

Schwessinger B, Roux M, Kadota Y, Ntoukakis V, Sklenar J, Jones A, Zipfel C. 2011. Phosphorylationdependent differential regulation of plant growth, cell death, and innate immunity by the regulatory receptor-like kinase BAK1. PLoS Genetics 7: e1002046-e1002046.

Segonzac C, Macho AP, Sanmartín M, Ntoukakis V, Sánchez-Serrano JJ, Zipfel C. 2014. Negative control of BAK1 by protein phosphatase 2A during plant innate immunity. The EMBO Journal 33: 2069-2079.

Stamatakis A, Hoover P, Rougemont J. 2008. A rapid bootstrap algorithm for the RAxML web servers. Systematic Biology 57: 758-771.

Tameling WIL, Baulcombe DC. 2007. Physical association of the NB-LRR resistance protein Rx with a Ran GTPase-activating protein is required for extreme resistance to Potato virus X. The Plant Cell 19: 16821694.

Thomas CM, Jones DA, Parniske M, Harrison K, Balint-Kurti PJ, Hatzixanthis K, Jones JD. 1997. Characterization of the tomato Cf-4 gene for resistance to Cladosporium fulvum identifies sequences that determine recognitional specificity in Cf-4 and Cf-9. The Plant Cell 9: 2209-2224.

Toufighi K, Brady SM, Austin R, Ly E, Provart NJ. 2005. The botany array resource: e-northerns, expression angling, and promoter analyses. The Plant Journal 43: 153-163.

van der Hoorn RAL, Laurent F, Roth R, de Wit PJGM. 2000. Agroinfiltration is a versatile tool that facilitates comparative analyses of Avr9/Cf-9-induced and Avr4/Cf-4-induced necrosis. Molecular Plant-Microbe Interactions 13: 439-446.

Voinnet O, Rivas S, Mestre P, Baulcombe DC. 2015. Retraction: An enhanced transient expression system in plants based on suppression of gene silencing by the 19 protein of tomato bushy stunt virus (retraction of The Plant Journal 33: 949, 2003).

Wang X, Kota U, He K, Blackburn K, Li J, Goshe MB, Huber SC, Clouse SD. 2008. Sequential transphosphorylation of the BRI1/BAK1 receptor kinase complex impacts early events in brassinosteroid signaling. Developmental Cell 15: 220-235.

Wang Z, Meng P, Zhang X, Ren D, Yang S. 2011. BON1 interacts with the protein kinases BIR1 and BAK1 in modulation of temperature-dependent plant growth and cell death in Arabidopsis. The Plant Journal 67: 1081-1093.

Zipfel C, Kunze G, Chinchilla D, Caniard A, Jones JD, Boller T, Felix G. 2006. Perception of the bacterial PAMP EF-Tu by the receptor EFR restricts Agrobacterium-mediated transformation. Cell 125: 749-760. 


\section{Supplemental data}

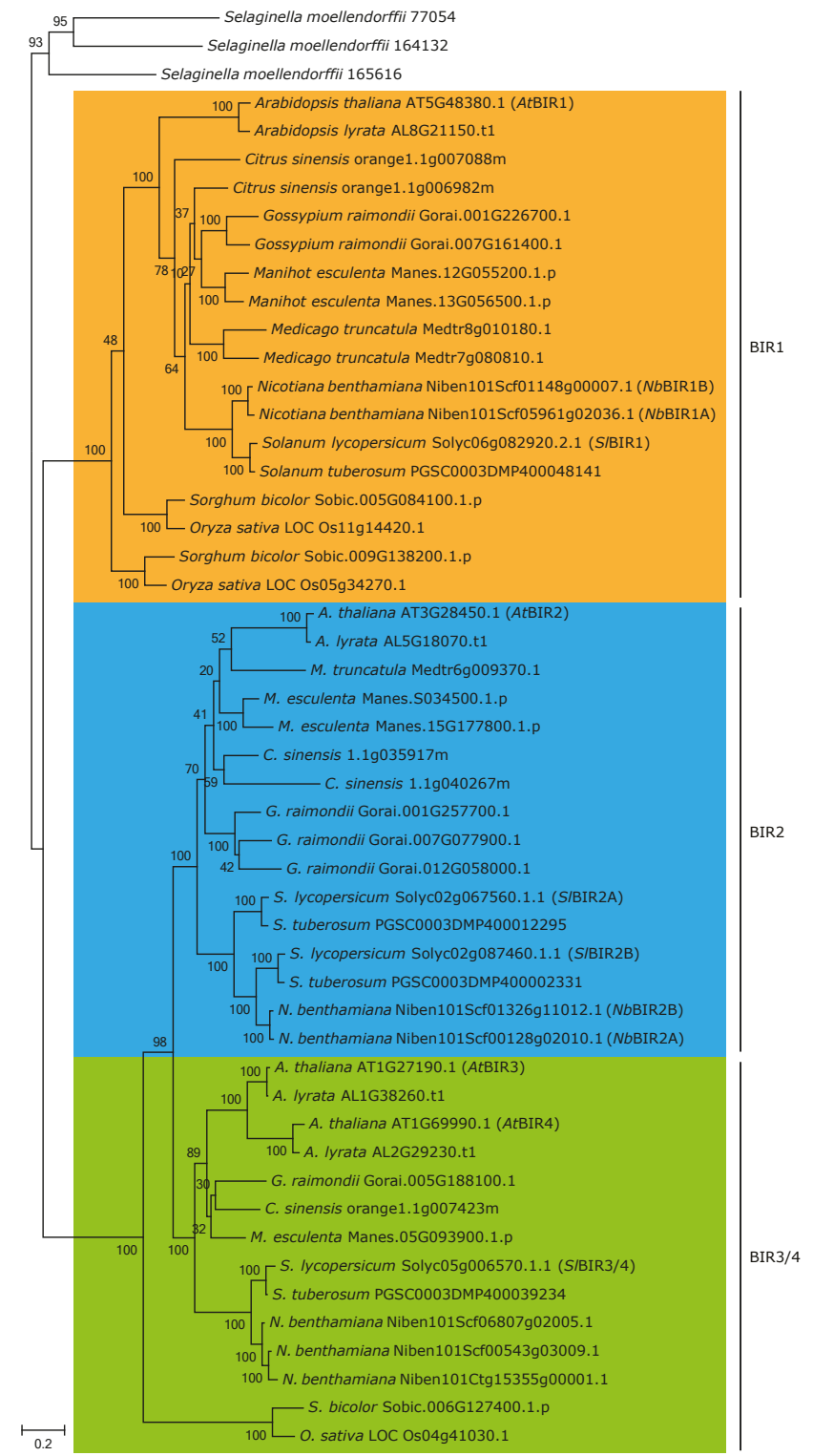

Figure S1. The BIR protein family is conserved among a wide range of plant species. Phylogenetic tree of protein sequences of AtBIR1 (orange), AtBIR2 (blue), and AtBIR3/4 (green), and their homologues in various plant species. Plant species, with a well-annotated genome, were selected with the aim to represent a diverse set of plants from the Plant Kingdom. Bootstrap values are indicated. The branch length represents the genetic change. See also Fig. 1A. 

SIBIR1 TTCTGTTGCCAAAGCTCCCGAGACCTT TAAGGGGAAT T TGGTGGAATGGATCACACA NbBIRIA CTCTGTCACCAAAGCTCCCGAGACCTTTAAGGGAAATCTCGTGGAATGGATT TCAAA NbBIR1B CTCTGTCACCAAAGCTCCCGAGACCTTTAAGGGAAATCTCGTGGAATGGATT TCAAA ${ }_{1}^{1670} \quad{ }_{1680}^{1690} \quad 1^{1700} \quad 1^{1710} \quad{ }^{1720}$ SIBIR1 ACTCTCTGGTGAATCTAAGCTTCAAGATGCGATTGACCATTCTCTGTCTAGTAAAGGTTA NbBIRIA T C T C T C TGG TGAAT C TAAG C T TCACGATGCGAT TGAC CAT T CG T TGTCTGGTAAAGGT TA NbBIR1B TCTCTCTGGTGAATCTAAGCT TCACGATGCAAT TGACCAT TCGTTGTCTGGTAAAGGT TA 1730 1740 1760 1780

SIBIR1 TGACAGTGAGATCTTCCAGGT'CCTTAAAGTTGCTTGTCGATGTGTGTTGTCTGCTGCTCC

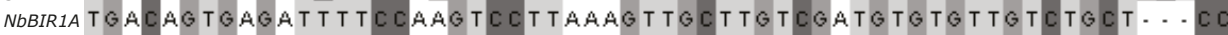
NbBIR1B TGACAG TGAGATT T TCCAAGTCCTTAAAGT TGCT TGTCGATGTGTGTTGTCTGCT . . CC SIBIR1 TAAGGAGAGGCCAACAATGT T TGAACTG TACCAGCT TC TGAGAG C CAT TGGAGAGCGGTA NbBIRIACAAGGAGAGGCCGACAATGTTTGAAGTGTACCAGTTTCTGAGAGCCATCGGAGAGCGATA NbBIR1B TAAGGAGAGGC CGACAATGTTTGAAGTGTACCAGTTTCTGAGAGCCATCGGAGAGCGATA 1850 1860 1870 1880 1890 SIBIR1 TCATT TCACAACTGATGATGACATT . . A ATGATGCCT . . GAGAGTGAT . . AGCGGAT NbBIRIA T CAT T T CACAACTGAAGATGACATT T TGATGATGCCTTCAGAAAGTGATGATGGCGGAC NbBIR1B TCAT T T CACAACTG AAGATGACATT T TGATGATGCCTTCAGAAAGTGATGATGGCGGAC SIBIR2A TGCT TAATGATAATAAGCT T T C TGGTAATATACCACCT CAGT T T T C TAGT T TGGGGAG SIBIR2B CGCTCAGTGATAACAAGCT TACTGGAAATAT T C CT TCTGAAT T T T T TAGT T TAAGTAG NbBIR2A TGCT TAATGATAACAAACT T TCTGGAAATAT TC C C C C TGAAT T T T C TAG T T TCAGTAG

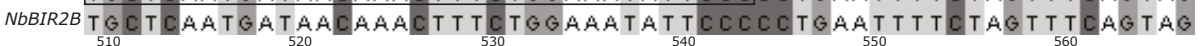
SIBIR2A GCT TAAGATATT T T C TGTCGCAAACAATGATCT T T CTGGTAGGATTCCGGAAGCT T T TGA SIBIR2B GCT TAATAGT T T T T CTGTGGCAAATAATCAACTCTCCGGTAGAATCCCCGCAGCT T TCGA NbBIR $2 A G C T$ CAAAACT T TAT C TG TGGCAAACAAT CAACT T T C CGGTAGAAT TC CGGCAGCT T TCGA

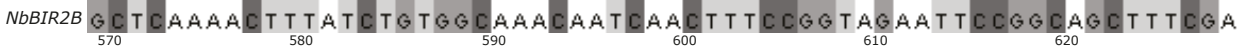
570
1

SIBIR2A T TCGGCTGATTCTTTTGATTTTGGAGGAAATGATGGGCTTTGTGGTGGTCCGTTGGGGAA SIBIR2B T TCGTCTAAGTT-..TAATT T TGAAGGAAATAGT- C T T TGTGGTGGACCT T TGGGGAA NbBIR2A CT CGGCCGATTCGGCTAATT T TGAGGGAAATAGTGGACTCTGTGGTGGACCTTTGGGGAA

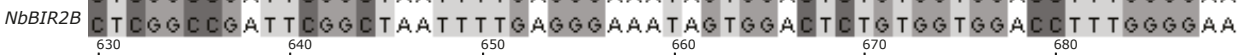
SIBIR2A ATGTGGGAGGCTTAGTAAGAAAAATT TAGCTATTATTATTGCTGCTGGTGTGTTTGGTGC SIBIR2B ATGTGGAGGTCTAAGTAAGAAAAGCTTAGCTATTATTATTGCAGCAGGAGTCTTTGGTGC NbBIR2A ATGTGGAGGACTCAGTAAGAAAAACT TAGCTATTATTAT TGCGGCAGGTGTCTTTGGTGC NbBIR2B A TG TGGAGGACT CAGTAAGAAAAACT TGGCTATTAT TAT TGCGGCAGGTGTCT T TGGTGC

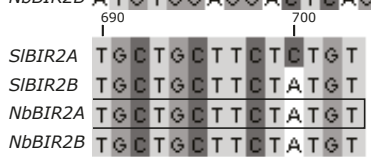

Figure S2. The VIGS constructs pTRV2:BIR1 and pTRV2:BIR2 target the intended BIR sequences of $N$. benthamiana and likely also the sequences of tomato. (A) An alignment of the VIGS target region of pTRV2:BIR1 (1613-1905, based on numbering of NbBIR1A) shows the homology of SIBIR1, NbBIRTA, and NbBIR1B. (B) An alignment of the VIGS target region of pTRV2:BIR2 (452-703, based on numbering of $N b B I R 2 A$ ) shows the homology of SIBIR2A, SIBIR2B, NbBIR2A, and NbBIR2B. The primer sequences that were used to clone the VIGS constructs are indicated with black boxes. 


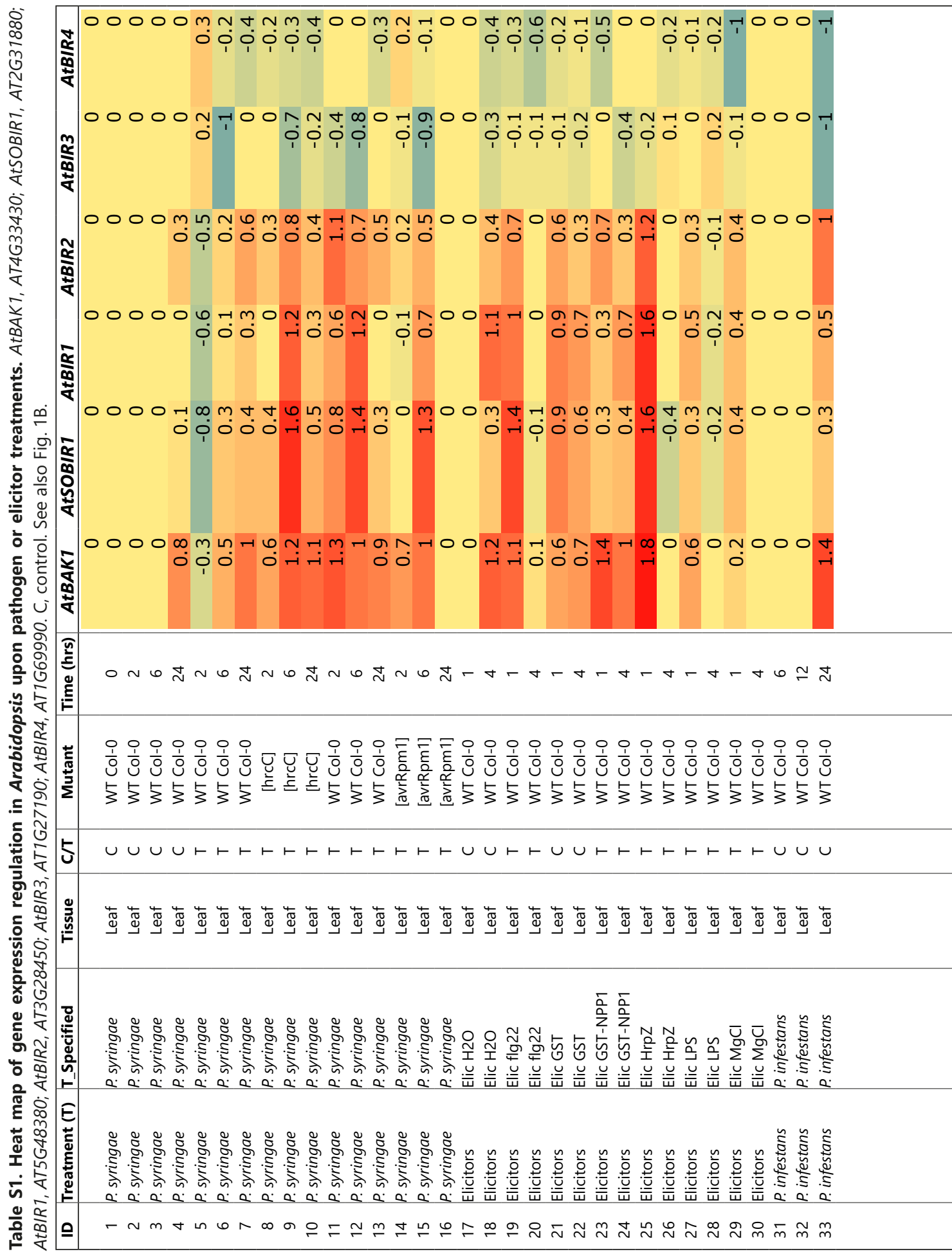




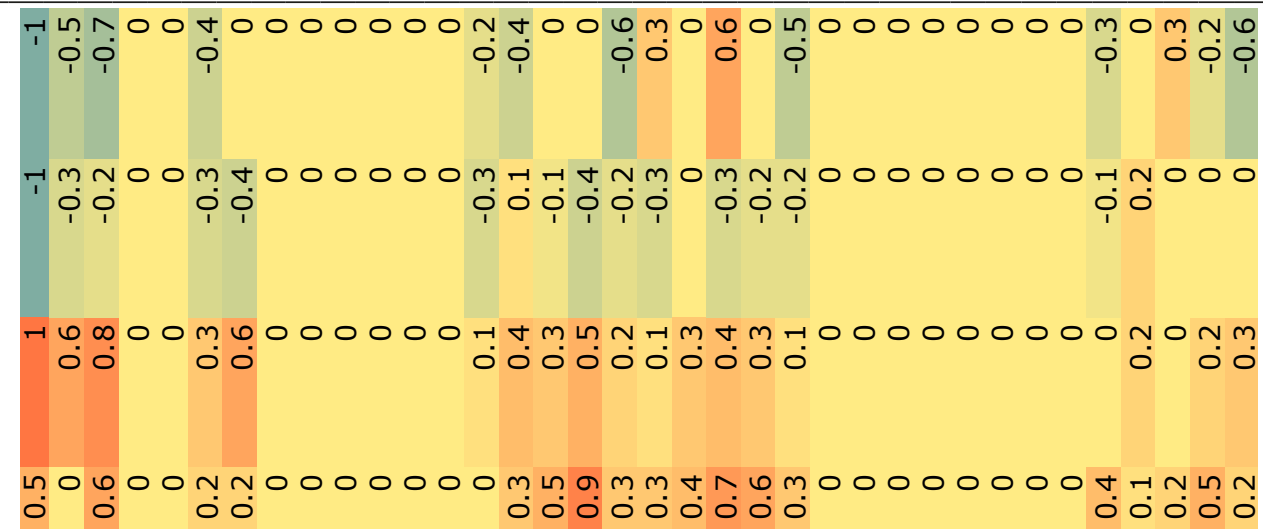

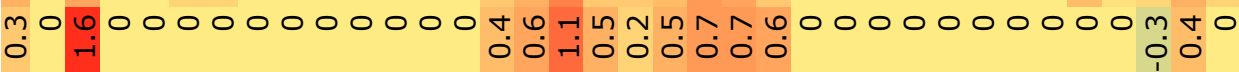

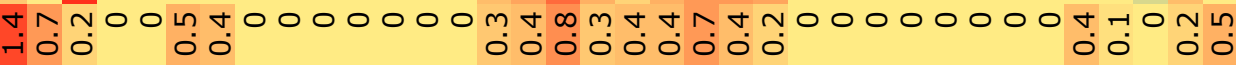
ம d

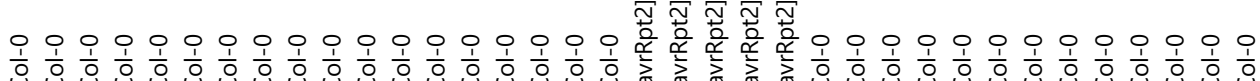

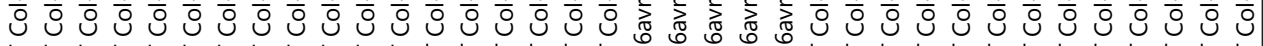

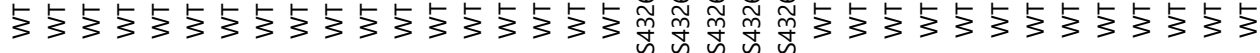
㟕出出

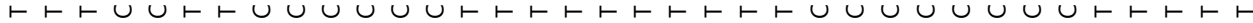

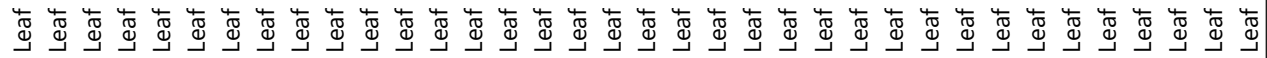




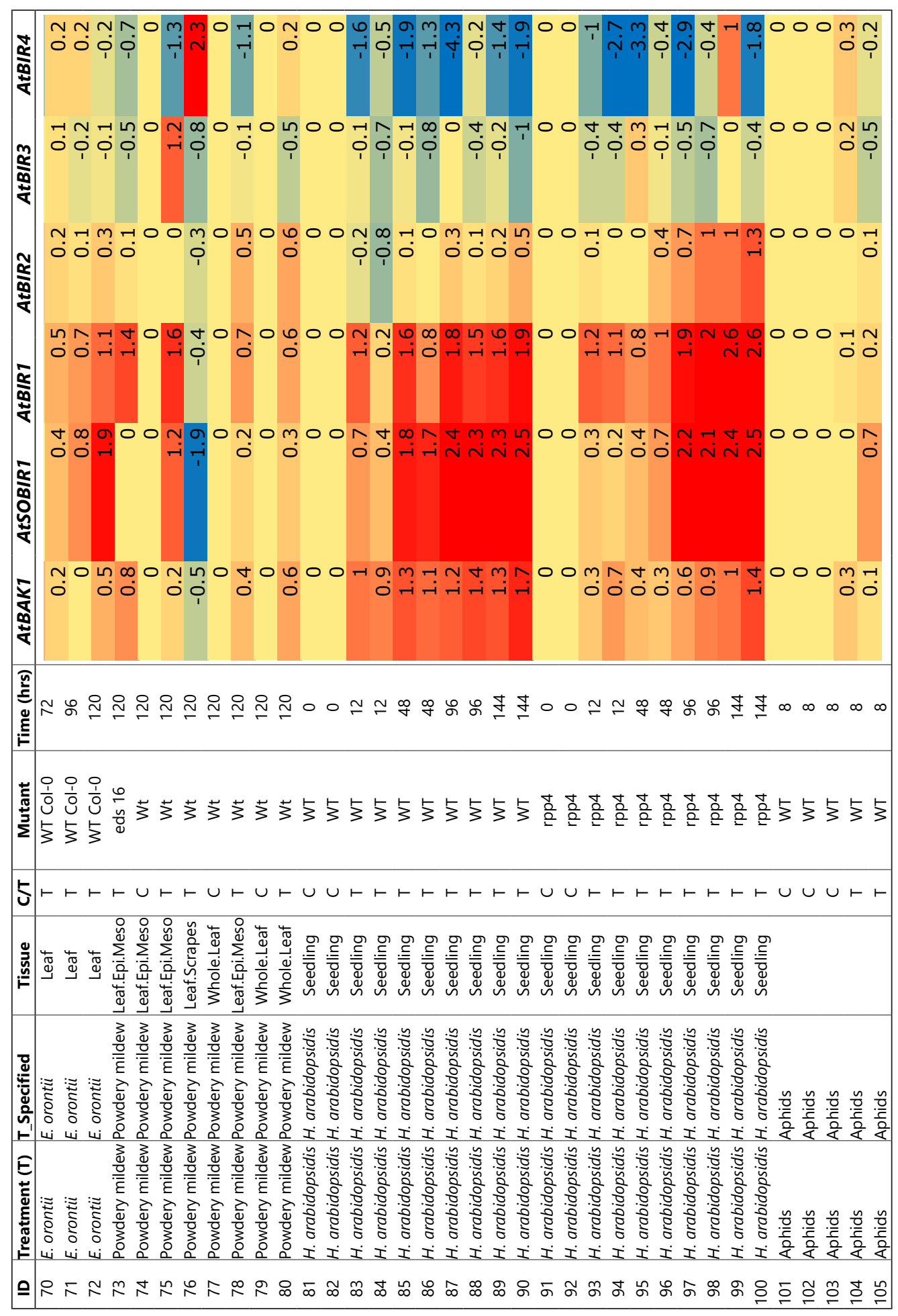


Table S2. Nucleotide sequences of the primers used for cloning and RT-PCRs. The restriction and gateway sites for cloning are indicated with small letters.

\begin{tabular}{|c|c|}
\hline Primer name * & Sequence \\
\hline NbBIR1AB_sil_fw & tctagaCTCTGTCACCAAAGCTCCCG \\
\hline NbBIR1AB_sil_rv & ccatgggctagcGTCCGCCATCATCACTTTCTGAAGG \\
\hline NbBIR2AB_sil_fw & tctagaTGCTCAATGATAACAAACTTTCTGGAAATATTCCC \\
\hline NbBIR2AB_sil_rv & ccatgggctagcACATAGAAGCAGCAGCACCAAAAGA \\
\hline SIBIR1_fw & caccATGGCTTTGGATAAGAGAGCTCTTACTACCTTTGC \\
\hline SIBIR1_nonstop_rv & TTGAGCAACAATGAGCTCGTCCATTTGG \\
\hline SIBIR2B_fw cacc & caccATGGCTCGTTTCAGATTTCTCCTTTCG \\
\hline SIBIR2B_nonstop_rv & TTGAGCAACAATGAGCTCGTCCATTTGG \\
\hline NbBIR1-864fw & TGAGAAGTCTGCTTCAAAAATGAG \\
\hline NbBIR1-1191rv & СССТTCACTCACCGAATGTAAC \\
\hline NbBIR2-816fw & TCACAAGCTCACTCAGGTTATGTTG \\
\hline NbBIR2-1193rv & TAGCAGCACCCAGACCAATTC \\
\hline $\mathrm{NbBIR3/4-833fw}$ & GAGCTTTTAAGCTTGTTCAGGTG \\
\hline $\mathrm{NbBIR3/4-1281rv}$ & ATGAAGCCAAGCAAGACCTC \\
\hline N259-ActinFw_am8 & TATGGAAACATTGTGCTCAGTGG \\
\hline N260-ActinRv_AM9 & CCAGATTCGTCATACTCTGCC \\
\hline
\end{tabular}

* fw, forward; rv, reverse 



\section{Chapter 7}

General discussion 


\section{Thinking in- and outside the box}

To improve our understanding of nature, the use of models has been embraced. Models help to explain, describe, and simplify biological processes. By simplifying systems that are in fact truly complicated, we are able to grasp the essence of a process. Nevertheless, models should be constantly changed and updated, when new knowledge on the studied system is gained. Furthermore, models should not restrict scientists from acquiring knowledge on new aspects that initially might not fit in the established model.

Here, the different models that are currently used in the field of plant-microbe interactions, to explain the molecular background of the constant battle between plants and pathogens, will be discussed. Light will be shed on the strong and weak points of these models, and a conclusion with recommendations for the future will be provided.

\section{A brief history of models describing plant-microbe interactions}

\section{The breeding point-of-view on plant-microbe interactions}

Plant breeding has been a human practice since thousands of years (Gross \& Olsen, 2010; Meyer \& Purugganan, 2013). Breeding focusses on crop qualities like a higher yield, tastier fruits, and increased disease resistance.

Most plants are resistant to most pathogens. If an entire plant species is resistant to all variants of a certain pathogen, this is called non-host resistance (NHR) (Heath, 1981). Several mechanisms have been shown to underlay NHR (Stam et al., 2014; Lee et al., 2017), so this umbrella-term should rather be used as a general phenomenon, and should not be used to explain a mechanism.

The gene-for-gene model describes the battle between plants and pathogens from a breeding point-of-view (Flor, 1942; Flor, 1971). In this battle, during co-evolution between plant and pathogen, compounds from the pathogen are recognized by the plant, leading to resistance. The gene of the pathogen that codes for the recognized compound is referred to as Avirulence gene (Avr). Recognition by the plant is based on the presence of a resistance $(R)$ gene. For each functional $R$ gene present in the plant, there is a matching Avr gene in the pathogen. A plant-pathogen interaction in which matching $R$ and Avr genes are present is referred to as 'incompatible'. When either a particular strain of the pathogen does not carry the matching Avr gene, and/ or a certain plant genotype does not carry the matching $R$ gene, the pathogen can infect the plant. This situation is called a 'compatible' interaction (de Wit, 1995).

Obviously, for the pathogen it has no benefit to be recognized by the host plant, so the intrinsic function of Avr genes of pathogens cannot be their recognition by plants. Indeed, many Avr genes have a virulence function, and they promote 
colonisation of susceptible plants, thereby benefiting the pathogen (de Wit et al., 2009; Khan et al., 2018). For this reason, Avr genes are also called virulence (Vir) genes. At the time the gene-for-gene model was introduced, the nomenclature was logical. Nowadays, most aspects are still valid, especially in terms of plant breeding. However, current advances in the research on plant-pathogen interactions have made the terminology 'Avr' confusing.

\section{The mechanistic point-of-view on plant-microbe interactions}

In the following years, molecular mechanisms that underly pathogen recognition and disease resistance were starting to be unravelled. This lead to the development of the so-called 'zigzag model' in 2006 (Jones \& Dangl, 2006). This model describes the evolutionary battle between plant and pathogen from a molecular point-of-view. The zigzag model clarifies the different layers of attack by the pathogen, and the different layers of defence by the plant.

The first layer of plant defence, according to the zigzag model, involves the recognition of conserved structural molecular patterns of the pathogen, called microbeor pathogen-associated molecular patterns (MAMPs or PAMPs), or damage-associated molecular patterns (DAMPs) that originate from the plant upon damage caused by a pathogen. As all microbes produce MAMPs, including non-pathogenic microbes, the term MAMP is preferred over the term PAMP. These intrinsic structures are recognized in the apoplast by cell surface-localized pattern recognition receptors (PRRs). A wellknown example of a MAMP is the peptide flg22, which derives from bacterial flagellin. This peptide is recognized by the PRR Flagellin-Sensing 2 (FLS2), which is a plasmamembrane (PM)-localised receptor-like kinase (RLK) (Gómez-Gómez \& Boller, 2000). Recognition of a MAMP leads to MAMP-triggered immunity (MTI), also referred to as PAMP-triggered immunity (PTI). To combat MTI, successful specialised pathogens have evolved effector proteins of which the expression is specifically induced in planta. These effector proteins are secreted into the apoplast or the cytoplasm, and interfere with MTI to lead to effector-triggered susceptibility (ETS).

The second layer of recognition is provided by resistance $(R)$ proteins that are able to recognise these effectors, allowing the plant to mount effector-triggered immunity (ETI). These R proteins can be PM-localized receptors, similar to PRRs, or cytoplasmic nucleotide binding leucine-rich repeat (NB-LRR) proteins. R proteins can either recognize effectors directly or indirectly, by guarding the host virulence target of the effector and sensing the manipulation of the target by the effector (Kourelis \& van der Hoorn, 2018). An example of direct recognition in the cytoplasm is the NB-LRR protein Rx from potato recognizing the coat protein from potato virus X (PVX) (Bendahmane et al., 1999). The example provided by RPM1-Interacting Protein 4 (RIN4) follows the guard model, as RIN4, which is targeted by multiple bacterial effectors, is guarded by several NB-LRRs (van der Hoorn \& Kamoun, 2008). ETI generally is a stronger response than MTI, and often culminates in the hypersensitive response (HR) (Jones \& Dangl, 2006). 
The zigzag model clearly describes the evolutionary arms race between pathogens and host plants. However, new insights into the mode of action of plant receptors, and pathogen MAMPs and effectors, has blurred the dichotomy between MTI and ETI (Boller \& Felix, 2009; Boller \& He, 2009; Thomma et al., 2011). Furthermore, the zigzag model is often (mis-)used. The terms MTI and ETI are for example (mis-)used to describe the resistance response based on pathogen recognition in the apoplast, and pathogen recognition in the cytoplasm, respectively (Dodds \& Rathjen, 2006; Peng et al., 2018). However, several PM-localized receptors do trigger a strong HR and recognise typical effectors. An example is the $\mathrm{Cf}-4$ resistance protein of tomato that recognizes the Avr4 effector of C. fulvum, as Avr4 is a typical fungal effector and the recognition event causes a strong HR. Another example is the tomato I protein, which is an RLP that provides resistance to Fusarium oxysporum f. sp. lycopersici carrying the apoplastic effector Avr1 (Catanzariti et al., 2017). Furthermore, some MAMPs are only narrowly distributed and have effector-like properties, whereas some effectors are so widely occurring that they might be considered to be MAMPs (Thomma et al., 2011). For instance, the bacterial MAMP eMAX, for enigmatic MAMP of Xanthomonas, seems to be only conserved in Xanthomonas (Jehle et al., 2013b). On the other hand, fungal Lysin-motif (LysM) effectors, like ECP6 from C. fulvum, seem to be widely occurring amongst fungi to sequester chitin (de Jonge et al., 2010). Likewise, homologues of the apoplastic effector Avr4 from C. fulvum have been found in other fungi and these are also recognised by Cf-4 (Stergiopoulos et al., 2010; Mesarich et al., 2015; Kohler et al., 2016). The continuum that is in fact present between MAMPs and effectors, tempted Thomma and co-authors to introduce the term MAMP-triggered susceptibility (MTS), to stress the fact that also MAMPs can be involved in provoking susceptibility in plant-microbe interactions (Thomma et al., 2011). Subsequent recognition of a MAMP in its turn can result in MTI of which the strength could well pass the threshold for HR (Thomma et al., 2011).

In 2014, the term effector-triggered defence (ETD) was proposed as another addition to the zigzag model (Stotz et al., 2014). ETD was defined as the defence responses triggered upon recognition of apoplastic effectors by receptor-like proteins (RLPS), which constitutively associate with the RLK Suppressor Of BIR11/Evershed (SOBIR1/EVR, further referred to as SOBIR1) (Liebrand et al., 2013). However, this subdivision does not hold with the discovery that not all RLPs trigger a similar response upon their activation. Some RLPs, for instance Cf-4, trigger a strong $H R$ response upon ligand recognition that is indistinguishable from cytoplasmic NBLRR-triggered responses (Liebrand et al., 2013), whereas other RLPs, like RLP23, only activate weak defence responses that are similar to MTI (Albert et al., 2015).

Also the term apoplastic immunity (AI) (Doehlemann \& Hemetsberger, 2013) did not provide a satisfactory improvement of our understanding of plant defence mechanisms. The authors nicely evaluated all interactions that take place in the plant 
during pathogen invasion, leading to the activation of defence responses. However, the term Al seems to imply that immunity is in fact established in the apoplast. However, for the immune response to be successful, after pathogen recognition in the apoplast, downstream signalling inside the cell is essential (Doehlemann \& Hemetsberger, 2013). So this type of immunity is not strictly apoplastic, and the term Al does not hold.

To accommodate all possible MAMPs, DAMPs, and effectors, the broadly including term invasion pattern (IP) was proposed for these host-recognised compounds in the so-called 'invasion model' (Cook et al., 2015). In this model, it is proposed to generalise and state that recognition of IPs by IP receptors (IPRs) leads to IPtriggered responses (IPTRs). The broad term IP even includes manipulated plant virulence targets (VTs), double-stranded RNA from viruses, and molecular signals from arbuscular mycorrhizal fungi (myc-factors) and nitrogen-fixing rhizobia (Nodfactors) that initiate symbiosis (Zipfel \& Oldroyd, 2017). IPTR may lead to successful defence (the end of symbiosis) or to a symbiosis with the invading microbe, which can be beneficial for both plant and microbe or only for the microbe (disease). Hereby this model includes both beneficial and pathogenic plant-microbe interactions. Successful suppression of IPTR, by IPs that function as effectors, allows continued symbiosis for biotrophic pathogens, and may cause additional IPs to be recognised by novel IPRs. Necrotrophic pathogens in their turn exploit IPTRs, especially when cell death is involved, and thereby continue their symbiosis with the plant (Cook et al., 2015).

Although the zigzag model is still very useful to describe the evolutionary battle between plants and pathogens, the zigzag model makes too narrow distinctions, whereas the invasion model is very broad. According to the invasion model, any molecule that can potentially be recognized by an IPR is an IP. This broad inclusion might be sufficient for studies approaching from the pathogen side, but for studies focusing at the plant side, the invasion model lacks differentiating possibilities. This might be a reason why, so far, this model has not yet been widely adopted in publications about pathogen recognition by plants. Here it is proposed, as an addition to the invasion model, to include the location of the recognition of the IP, which can be either extracellularly (for extracellular IPS, ExIPs) or intracellularly (for intracellular IPs, InIPs) (Fig. 1). Introducing this spatial dichotomy aims to facilitate a more clear nomenclature for the type and location of recognition of IPs by different plant receptors upon pathogen attack. To keep it as simple as possible, and because the terms IPR and IPTR will become too long when the location is added, in this 'spatial invasion model' cell surface receptors, which recognize ExIPs, will be referred to as transmembrane (TM)-receptors, whereas cytoplasmic receptors recognize InIPs. 


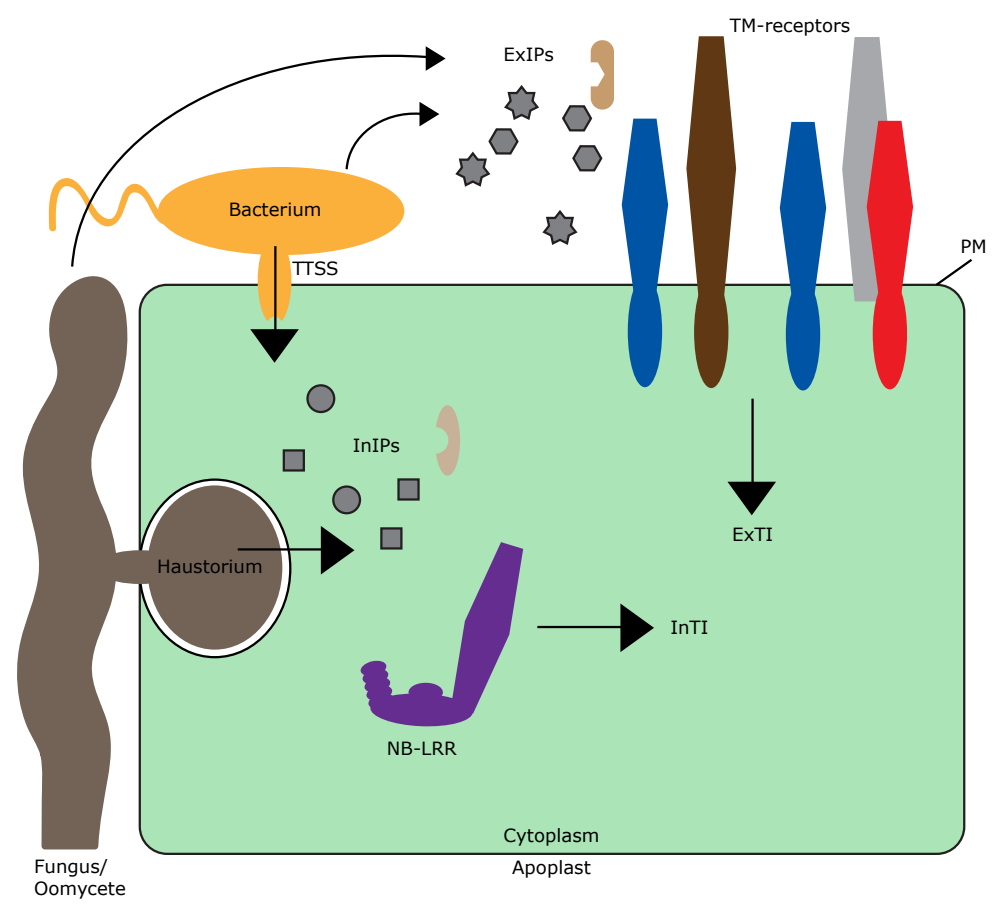

Figure 1. Schematic overview of the 'spatial invasion model'. Extracellular invasion patterns (ExIPs) which accumulate upon invasion of the plant by microbes, are sensed by TM-receptors that are present on the PM. ExIPs can be pathogen-derived MAMPs and effectors, or host-derived DAMPs (all shown as grey structures) and VTs (light brown). Intracellular localised IPs (InIPs) are sensed by cytoplasmic NBLRR receptors. InIPs can be pathogen-derived molecules (shown as grey structures inside the cell), or host-derived VTs (light brown). Both extracellular and intracellular recognition leads to the activation of defence responses, referred to as extracellularly- and intracellularly-triggered immunity (ExTI and $\operatorname{lnTl}$ ), respectively. Picture inspired by Dodds \& Rathjen (2010). PM, plasma membrane; MAMP, microbeassociated molecular pattern; DAMP, damage-associated molecular pattern; TTSS, type three secretion system; NB-LRR, nucleotide binding leucine-rich repeat; VT, virulence target.

\section{The spatial dichotomy: extracellularly- versus intracellularly-triggered immunity (ExTI and InTI)}

BAK1 recruitment and other common themes in extracellularly-triggered immunity Recognition of InIPs involves cytoplasmic receptors, usually NB-LRR proteins (Zhang et al., 2017), whereas recognition of ExIPs involves TM-receptors (Zipfel, 2014; Hohmann et al., 2017; Ranf, 2017; Chapter 1). The extracellular domain (ECD) of TM-receptors can carry different motifs and determines the recognition specificity of the receptor. Different ECDs facilitate the recognition of different types of ExIPs (Zipfel et at., 2014; Hohmann et al., 2017; Ranf, 2017). TM-receptors can be divided into RLKs and RLPs. Here, we focus on LRR-RLKs and LRR-RLPs, further referred to as RLKs and RLPs, which 
recognize various extracellular proteins and peptides (Li \& Tax, 2013; Wang et al., 2010; Zipfel, 2014; Ranf, 2017). RLPs lack an intracellular signalling domain and instead constitutively interact with the RLK SOBIR1 to form bimolecular RLKs (Liebrand et al., 2013 and 2014; Gust \& Felix 2014).

A common step after ligand recognition by RLKs is the recruitment of the coreceptor BRI1-Associated Kinase 1/Somatic Embryogenesis Receptor Kinase 3 (BAK1/ SERK3, further referred to as BAK1) and other members of the SERK family (Heese et a., 2007; Chinchilla et al., 2007; Chinchilla et al., 2009; Couto \& Zipfel, 2016; Hohmann et al., 2017). For example, for the well-studied RLK FLS2, BAK1 recruitment was shown upon treatment with the bacterial flagellin-derived peptide flg22 (Heese et al., 2007; Chinchilla et al., 2007). Overall, BAK1 recruitment is thought to lead to transphosphorylation events between the kinase domains of the ligand-activated RLK and BAK1, and subsequent initiation of downstream signalling (Wang et al., 2008; Schulze et al., 2010; Schwessinger et al., 2011; Oh et al., 2011; Macho et al., 2014, 2015; Macho \& Zipfel, 2014; Lu et al., 2010; Lin et al., 2013 and 2014). Dependency on BAK1 has been shown for a plethora of RLKs in several plant species (Table 1). Strikingly, also RLP/SOBIR1 bi-partite complexes have been shown to recruit BAK1 upon ligand perception by the RLP that is associated with SOBIR1, suggesting that RLP/SOBIR1 complexes function as true bimolecular RLKs (Albert et al., 2015; Postma et al., 2016; Wang et al., 2018; Domazakis et al., 2018). Additionally, many RLP-mediated immune responses have been described to depend on BAK1, although actual BAK1 recruitment has not yet been demonstrated for all of them (Table 1).

The formation of higher order complexes is a common mechanism, also for TMreceptors containing extracellular domains other than LRRs (Zipfel, 2014; Ranf, 2017). For instance the LysM-containing Chitin Elicitor Receptor Kinase 1 (CERK1), functions as a co-receptor for several TM-receptors with LysM ECDs in the perception of ExIPs like fungal chitin and bacterial peptidoglycan (PGN) (Ranf, 2017). However, here the focus lies on comparisons among TM-receptors with LRR ECDs.

In recent years, with the gain of more molecular detail on various plant-pathogen interactions, the previously made division between PRRs, which signal for MTI by recognizing MAMPs/DAMPs, and PM-localized immune receptors (R proteins), which signal for ETI by recognizing extracellular effectors, became blurry. Accumulating evidence indicated that such a strict separation could not be maintained (Thomma et al., 2011). Interestingly, molecular proof has now indeed become available that supports a continuum between these defence responses, as signalling by both PM-localized PRRs and $R$ proteins appears to eventually depend on the same mechanism, namely BAK1 recruitment (Albert et al., 2015; Postma et al., 2016). Furthermore, BAK1 recruitment is followed by trans-phosphorylation events between the two kinase domains that have come in close proximity, namely the kinase domain of BAK1 and either that of SOBIR1, or that of the ligand-binding RLK (Schwessinger et al., 2011; Wang et al., 2008; Chapter 3). 
Table 1. LRR-RLKs and LRR-RLPs involved in immunity, their ligands (IPs), and the involvement of SERKs and SOBIR1 in their signalling.

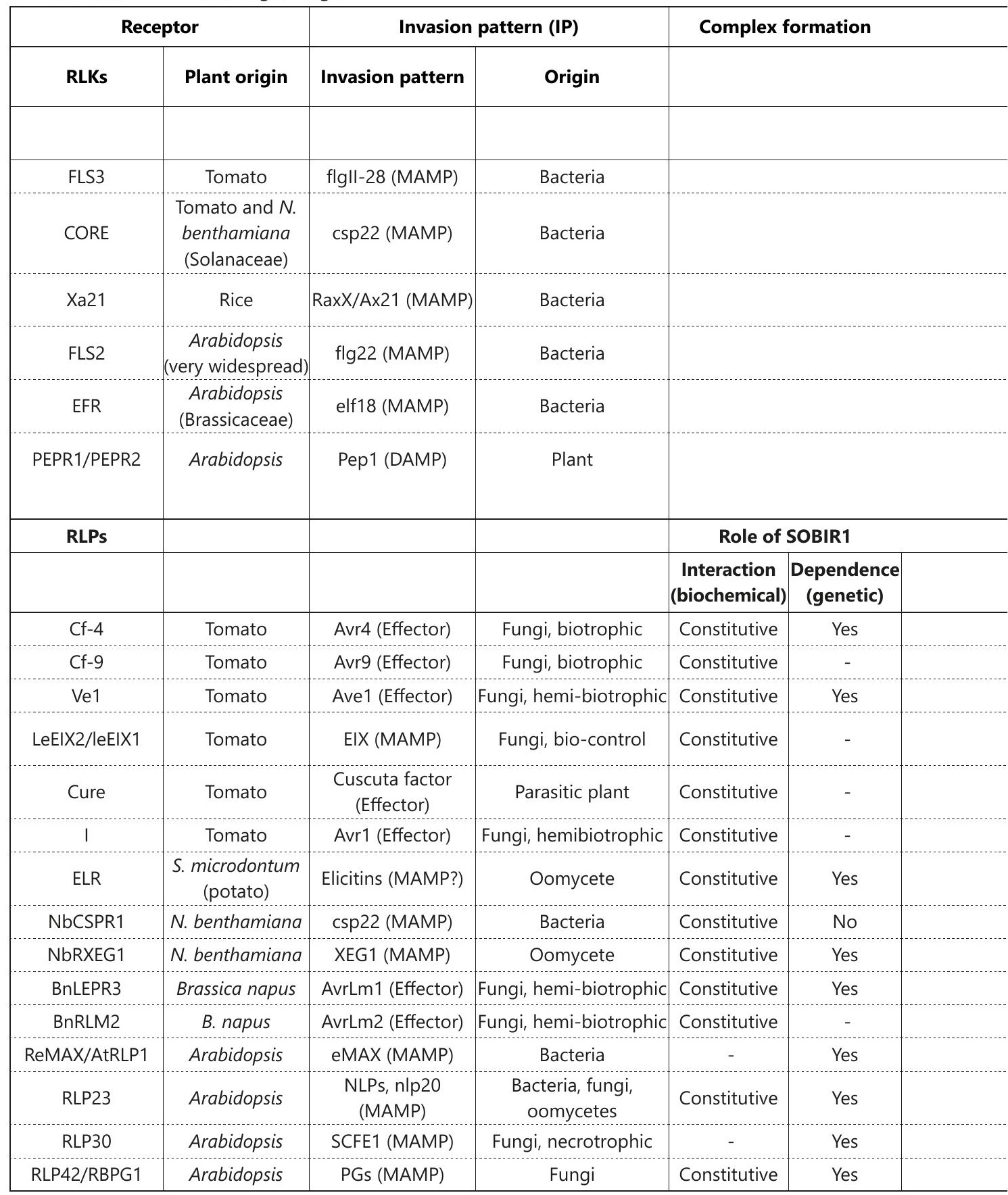




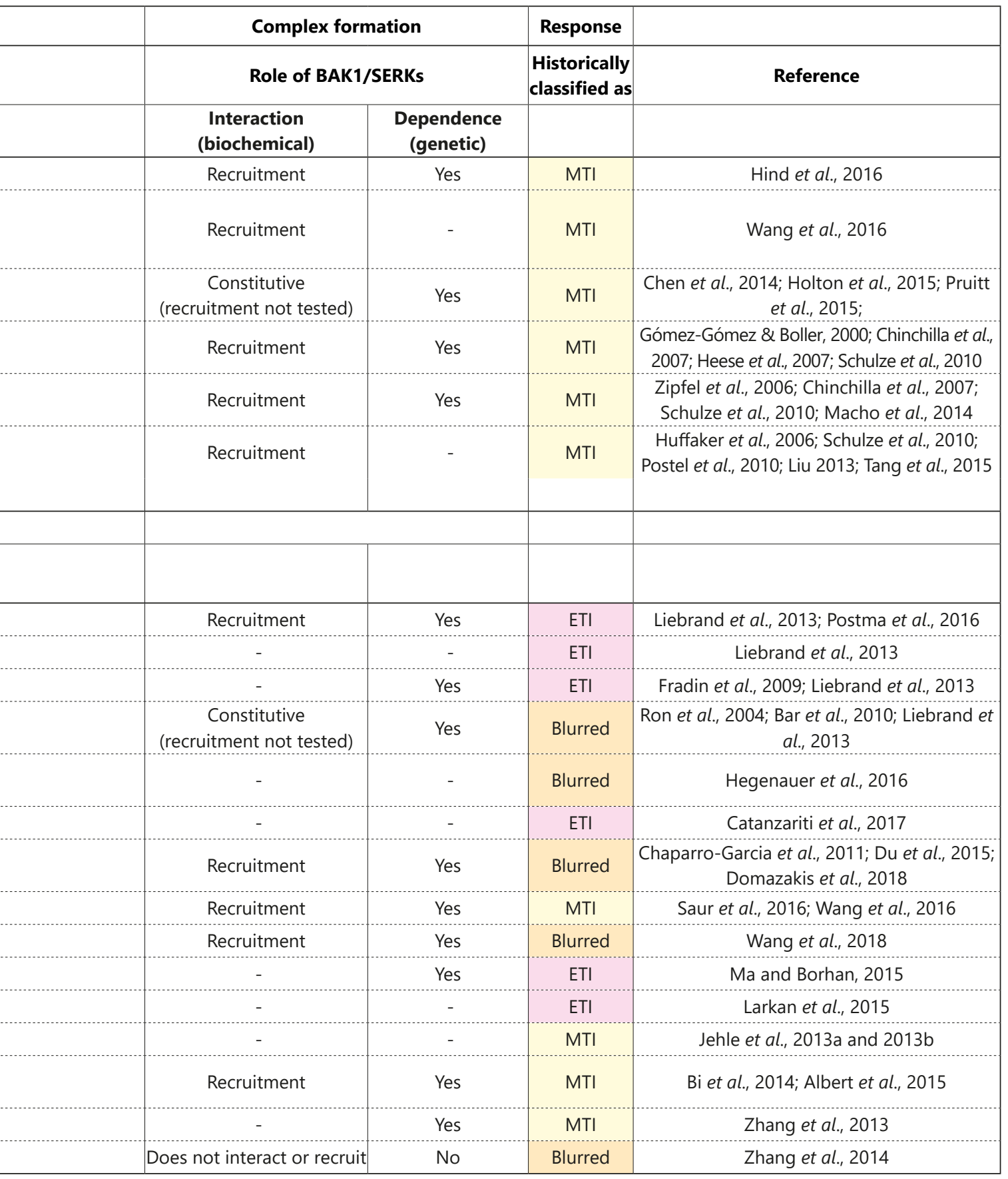


Remarkably, some RLPs historically classify as PRRs (triggering MTI), whereas others are considered to be $\mathrm{R}$ proteins (triggering ETI) (Table 1). For example Cf-4, the tomato RLP that recognizes the apoplastic effector Avr4 of C. fulvum, triggers a strong defence response upon Avr4 recognition, including an HR (Joosten et al., 1994; Thomas et al., 1997). The $R$ gene Cf-4 and the effector gene Avr4 are a classic example of a gene-forgene interaction (de Wit, 1995). On the other hand, RLP23 from Arabidopsis triggers only a basal defence response, including callose deposition and a swift burst of reactive oxygen species (ROS), but no HR (Böhm et al., 2014; Albert et al., 2015; Jones \& Dangl, 2006). RLP23 recognizes an epitope of Necrosis and Ethylene-inducing Peptide 1 (NEP1)-Like Proteins (NLPs) (Albert et al., 2015). NLPs are wide-spread proteins, occurring in bacteria, fungi, and oomycetes, rendering them typical MAMPs (Böhm et al., 2014). Interestingly, both the activation of the Cf-4/SOBIR1 complex and the RLP23/ SOBIR1 complex requires the recruitment of BAK1 (Postma et al., 2016; Albert et al., 2015). Likely, upon ligand recognition and this BAK1 recruitment, transphosphorylation of the kinase domains of SOBIR1 and BAK1 occurs in both complexes to activate downstream signalling events (Chapter 3). BAK1 recruitment is probably a general activation step for all RLP/SOBIR1 complexes, regardless of triggering either strong or basal defence responses (Table 1) (Liebrand et al., 2014; Gust \& Felix, 2014; Domazakis et al., 2018; Wang et al., 2018). Interestingly, ligand-recognizing RLKs provide their own specific kinase domain to facilitate transphosphorylation with BAK1, whereas ligandrecognizing RLPs in all cases provide the same SOBIR1 kinase domain. This suggests that the downstream responses triggered by RLP/SOBIR1 complexes will intrinsically be identical, as in all cases the same SOBIR1/BAK1 kinase domain combination will initiate downstream signalling. The differences in final defence output, namely a strong defence response including an $\mathrm{HR}$, versus a basal response, might be based on several variables as discussed in the next paragraph.

The striking finding that BAK1 recruitment is required for defence signalling by all LRR-containing TM-receptors tested thus far, tempts to argue that the recognition of any ExIP by any TM-receptor, despite the previous classification of TM-receptors into PRRs and R proteins (and their output into $\mathrm{MTI}$ and ETI, respectively), leads to the activation of similar defence responses. When we look further, BAK1 recruitment is not the only common phenomenon downstream of TM-receptors.

Receptor-like cytoplasmic kinases (RLCKs) provide the first signalling steps from TM-receptors into the cytoplasm (Ma, 2014; Liang \& Zhou, 2018). RLCKs have been shown to facilitate several signalling pathways downstream of RLKs, including a link to ROS production, to the mitogen-activated protein kinase (MAPK) cascade, and even to transcriptional reprogramming in the nucleus (Kadota et al., 2014; Li et al., 2014; Yan et al., 2018; Lal et al., 2018). Recent findings also show roles of RLCKs downstream of RLP/SOBIR1 complexes (Chapter 5; Wan, 2017). However, further research is necessary 
to clarify the proposed diverse negative and positive regulatory functions of RLCKs in downstream signalling mediated by RLP/SOBIR1 complexes.

The production of ROS is a swift general output of activated TM-receptors (May et al., 1996; Piedras et al., 1998; Kadota et al., 2014; Li et al., 2014). Also the typical $\mathrm{Ca}^{2+}$ spiking, the activation of a MAPK cascade, and the activation of $\mathrm{Ca}^{2+}$-dependent protein kinases (CDPKs) are common responses that are triggered by TM-receptors (Seybold et al., 2014; Lee et al., 2015; Stulemeijer et al., 2007; Romeis et al., 1999, 2000, and 2001).

Finally, activation of TM-receptors in all cases leads to transcriptional reprogramming with the aim to further activate defence, including the production of phytohormones, via the activation of WRKY transcription factors (Etalo et al., 2013; Pieterse et al., 2012; Li et al., 2016).

All these commonalities lead to argue in favour of the spatial update to the invasion model, that only differentiates between the location where recognition of the invading microbe takes place (Fig. 1). In this manner, we move away from using the zigzag model to differentiate between MTI and ETI that is triggered by extracellular MAMPs and effectors, respectively (Jones \& Dangl, 2006). As in practice, the term IP seems too broad to use in research about the recognition of invaders by plants, this update differentiates IPs based on their location. This spatial dichotomy will help to clearly describe plant-pathogen interactions. The division between extracellular and intracellular IPs will hold true, as this is a division based on biology, and not on the interpretation by scientists.

Although not discussed in detail here, TM-receptors with another ECD than LRRs, which likely do not depend on BAK1-recruitment, obviously also fit in the spatial invasion model (Fig. 1). Likewise, the different versions of recognition through guards and decoys are not mentioned here (Kourelis \& van der Hoorn, 2018). However, these different mechanisms of recognition are also included in the model, as these can be regarded as recognition of either ExIPs or InIPs (Fig. 1). Also the suppression of responses by effectors (Dodds \& Rathjen, 2010) is not shown in the model. However, this part of the evolutionary battle between plant and pathogen is covered, as ExIPs can suppress ExTI-related responses via defensive and offensive mechanisms in the apoplast (Stergiopoulos \& de Wit, 2009), and the same holds for InIPs that exert their virulence function in the cytoplasm (Khan et al., 2018). Of course, future findings may challenge this model again, and potentially lead to novel improvements.

\section{Possible causes of gradual differences between weak and strong ExTI responses}

Although all ExTI signalling goes via TM-receptors and includes the recruitment of co-receptors like BAK1, the responses that are triggered in this way have different intensities. There are strong responses that follow the classic ETI principle, like Cf4-triggered HR, and weak responses that follow the MTI principle, like FLS2- and 
RLP23-induced basal defence (Thomas et al., 1997; Gómez-Gómez \& Boller, 2000; Böhm et al., 2014; Jones \& Dangl, 2006). However, not only between TM-receptors classically referred to as PRRs and R proteins there are differences in output, but also among PRRs there are significant variations in intensity and timing of the generated defence outputs (Wan, 2017). A recent comparative study showed that the ROS burst upon treatment with similar amounts of flg22, nlp20 (the NLP epitope), or chitin differs in magnitude and timing (Wan, 2017). These differences in intensities might be explained by subtle differences that occur at one or more levels of the ExTI defence pathway.

ExIPs that are located in the apoplast might not all be equally stable. Differences in ExIP stability will at least partially dictate how many molecules of the compound are eventually being perceived by TM-receptors, and how fast and strong ExTI is triggered. For instance, instable variants of Avr4 have been shown to allow C. fulvum to evade Cf-4-mediated recognition and resistance (Joosten et al., 1997; van den Burg et al., 2003). Additionally, differences in the affinity of specific TM-receptors for particular ExIPs might be a factor determining the differences in the intensity of the defence responses that are generated (Hohmann et al., 2017; Hohmann et al., 2018).

Not only the stability of the ExIP, but also the stability of the matching TMreceptor might influence the intensity of ExTI. TM-receptor synthesis, recycling, and degradation have been shown to play an important role in regulating defence signalling. The pace by which these various processes take place will differ from one receptor to another (Postma et al., 2016; Frescatada-Rosa et al., 2015; Ben Khaled et al., 2015; Mbenguea et al., 2016; Lu et al., 2011). Differences in regulation at the level of receptor biogenesis, endocytosis, and breakdown might account for different intensities of the defence signal.

All RLPs tested so far interact with SOBIR1, and BAK1 recruitment seems to be a common mechanism for ExTI-related signalling (Table 1). Interestingly, for different RLKs, BAK1 recruitment provides a kinase domain that every time forms a different couple for every primary ligand-recognizing RLK. On the contrary, in the case of RLP/SOBIR1 complexes, recruitment of BAK1 upon ligand recognition by the RLP involved, in all cases leads to the same kinase domain couple; that of SOBIR1 and that of BAK1. So, what could cause the observed differences in intensity of downstream defence responses triggered by different RLPs? One obvious difference between these immune complexes is the short cytoplasmic tail of the RLP that is involved in the complex. These tails usually comprise less than 30 amino acids, and apart from the presence of a conserved Trp and Phe residue, these tails do not seem to have an obvious common motif (Gust \& Felix, 2014). So far, these cytoplasmic tails have not been studied in much detail. An ER-retention signal, consisting of the dilysine motif (KKRY), is present at the C-terminal end of both the Cf-4 and the Cf-9 protein, as Cf-4 
and Cf-9 are identical at their C-termini (van der Hoorn et al., 2001). However, this KKRY motif proved not to be essential for Cf-9 function and it was suggested that this motif might be masked by interacting proteins, of which one could be SOBIR1. In this way, only Cf-4/Cf-9 proteins constitutively interacting with SOBIR1 would not be retained in the ER and would properly localize at the PM (van der Hoorn et al., 2001). Swapping of the cytoplasmic tails of RLPs either signalling for basal ExTI or strong HR-associated ExTI, so for example between RLP23 and Cf-4, might point to a possible role of these short cytoplasmic tails in determining the strength of the defence signalling output.

SOBIR1 is only present as a single copy gene in Arabidopsis. In Solanaceous plants, there is an additional homologous gene present, referred to as SOBIR1-like, which seems to have a redundant function next to SOBIR1 itself (Liebrand et al., 2013). BAK 1 on the other hand, is also called SERK3, and is a member of the SERK family, consisting of five homologues in Arabidopsis. Also in Solanaceous plants and for example rice, several SERK homologues have been annotated (Liebrand et al., 2014). Possibly, differential preference for certain SERK proteins in specific defence signalling pathways is a denominator to signal for either basal or strong immunity (Yasuda et al., 2017; Ma et al., 2016). In Cf-4 signalling for example, BAK1/SERK3 as well as SERK1 have been shown to be involved (Fradin et al., 2011; Postma et al., 2016). In RLP23 signalling even four SERKs, namely SERK1, SERK2, BAK1/SERK3, and BKK1/SERK4, have been shown to be involved (Albert et al., 2015). However, the precise roles and preferences for the different SERKs in various RLK- and RLPcomplexes and their possible effect on the strength of the signalling output, needs to be further elucidated. This is challenging, as redundancy makes it difficult to study the individual functions of the SERK family members. Additionally, not only the presence of different homologues of the SERKs, but also the presence of different amounts of SOBIR1 and SERKs might play a role. For instance, the co-immunopurifying band of SOBIR1 upon immunoprecipitation of Cf-4 is very intense, and SOBIR1 has been shown to form homodimers (Liebrand et al., 2013; Chapter 3). These results lead to argue that multiple SOBIR1 proteins might form a complex with $\mathrm{Cf}-4$. Possibly different amounts of SOBIR1 and SERKs associating with the RLP involved direct the intensity of the defence responses that are triggerd.

Pseudokinases, like BAK1-Interacting RLK 2 (BIR2), have been shown to negatively regulate the availability of BAK1 for its recruitment to activated signalling complexes (Halter et al., 2014). As different BIR homologues might differentially regulate the availability of different pools of BAK1 in diverse nanodomains (Chapter 6; Imkampe et al., 2017; Bücherl et al., 2017), this highly complex regulation could also contribute to the variety of ExTI intensity. 
One step further downstream, cytoplasmic RLCKs form a signalling hub that converges signals from TM-receptors to further intracellular downstream signalling partners (Kadota et al., 2014; Li et al., 2014; Eckardt, 2011; Couto \& Zipfel, 2016; Liang \& Zhou, 2018). It is likely that also downstream of RLP/SOBIR1-complexes RLCKs play a role. So far, no conclusive experimental data of RLCKs functioning downstream of RLPs have been published, although some hints have been revealed (Rowland et al., 2005; Abu-Qumar et al., 2008; Wan, 2017; Chapter 5). SOBIR1 possibly interacts with the Arabidopsis RLCK Botrytis-Induced Kinase 1 (BIK1) and several tomato RLCKs, and negative as well as positive roles for RLCKs in regulating RLP/ SOBIR1-mediated immunity have been proposed (Chapter 5; Wan, 2017). Possibly, differential phosphorylation of the same RLCK downstream of various RLPs explains the generation of differential downstream responses. Nevertheless, the RLCK family is big, highly diverse, and redundant, and has very diverse roles in defence as well as in development (Eckardt, 2011; Liang \& Zhou, 2018). Different RLCKs might contribute to further differentiation of immune responses downstream of TM-receptors.

Not only the amounts of available TM-receptors to be activated are regulated, but also the activity of these receptors (Couto \& Zipfel, 2016). The phosphorylation status of the kinase domain of TM-receptors and downstream components like RLCKs, CDPKs, and MAPKs is kept in check by various phosphatases. For example, BAK1 and BIK1 are kept in check in the resting state by Protein Phosphatase 2A (PP2A) and PP2C38, respectively (Segonzac et al., 2014; Couto et al., 2016). Also for the rice RLK Xa21, which confers resistance to the bacterial pathogen Xanthomonas oryzae pv oryzae secreting Ax21, a control mechanism consisting of de-phosphorylation by a PP2C member has been shown (Park et al., 2008). The affinity of different phosphatases for distinct immuno-complexes and downstream signalling pathways, in combination with their efficiency to de-phosphorylate the signalling partners, might also play a role in regulating the intensity of ExTI.

In conclusion, I here want to emphasise that with the introduction of the terms ExIP and InIP, the concept of MAMPs, DAMPs, and effectors is not abandoned. It is still essential to understand the origin and intrinsic role of the different ExIPs, and their position in the evolutionary battle between plants and pathogens. However, the terms ExIP and InIP serve as so-called umbrella-terms with the aim to improve and specify the invasion model, and facilitate a clear description of recognition of microbial invaders by plants. 


\section{The findings of this thesis in a broader perspective}

In this thesis, data are presented that move the field of molecular plant pathology a step forward (Fig. 2). This work builds on the recent discoveries that SOBIR1 is a regulatory RLK that interacts with RLPs (Liebrand et al., 2013), and that BAK1 is recruited to RLP/SOBIR1 complexes upon ligand elicitation (Albert et al., 2015; Postma et al., 2016). The work described in this thesis demonstrates that SOBIR1 is a key positive regulator of defence. SOBIR1-mediated immune responses are suppressed by the bacterial effector AvrPto (Chapter 2). Transphosphorylation of SOBIR1 and BAK1 is shown to be essential for immune signalling (Chapter 3), and specific phosphorylation sites of SOBIR1 were found that play a role in immunity (Chapter 4). Also the involvement of RLCKs downstream of SOBIR1 (Chapter 5), and the negative regulation by BIR1 in Solanaceous plants is shown (Chapter 6). In the following sections, these findings are placed into a broader perspective.

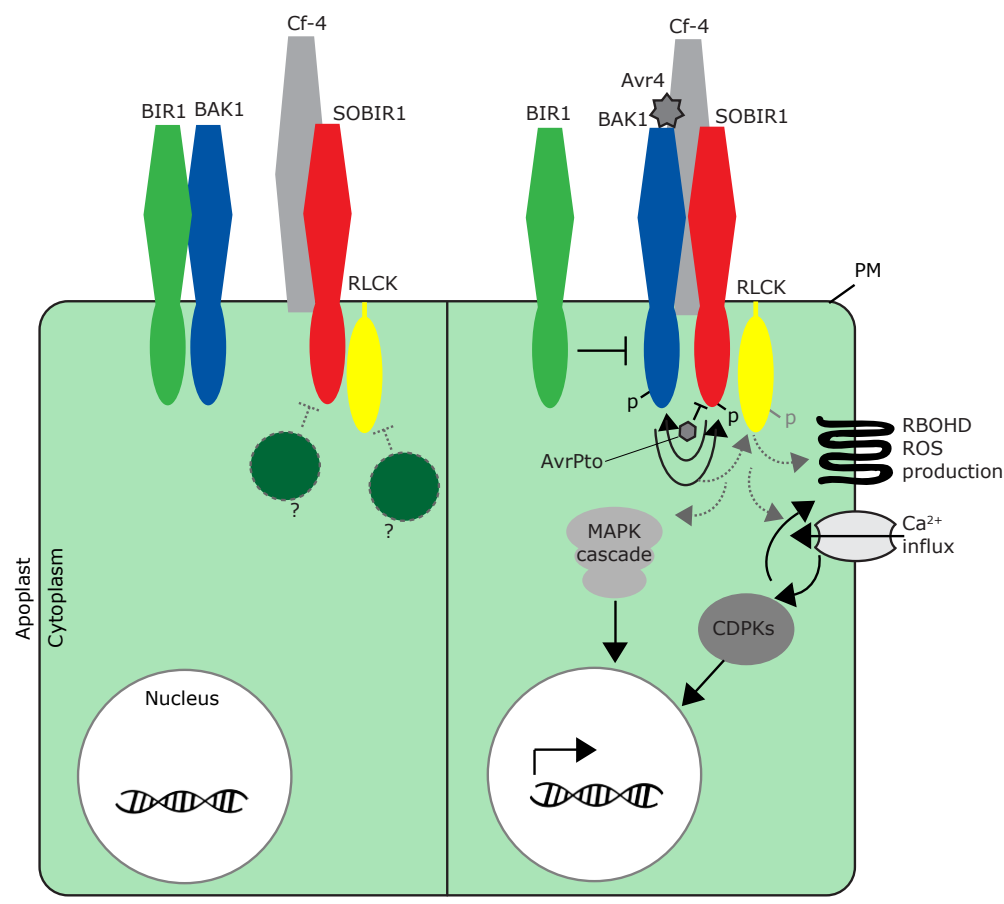

Figure 2. Schematic overview of the current model of immune signalling downstream of RLP/ SOBIR1-complexes, taking Cf-4 as an example. Cf-4 constitutively interacts with SOBIR1. In the resting state (left), BAK1 is sequestered from the Cf-4/SOBIR1 complex by BIR1 (Chapter 6). SOBIR1 interacts with one or several RLCKs (Chapter 5), and these kinases, in addition to the kinase domain of SOBIR1, are expected to be kept in check by phosphatases (dark green circles). Upon ligand (Avr4) recognition (right), BAK1 is recruited to the RLP/SOBIR1-complex, and transphosphorylation events take place between the kinase domains of BAK1 and SOBIR1 (Chapter 3 and 4), and phosphorylation with one or more RLCKs probably also takes place. SOBIR1 is targeted by the bacterial effector AvrPto (Chapter 2), 
resulting in the suppression of defence responses. Precise downstream steps are not clear yet, but ROS are produced and transcription is reprogrammed to activate defence gene expression via MAPKs and CDPKs. Predicted proteins and pathways are indicated with question marks and dotted/grey lines and arrows. PM, plasma membrane; BAK1, BRI1-Associated Kinase 1; BIR1, BAK1-Interacting RLK 1; ROS, reactive oxygen species; RBOHD, Respiratory Burst Oxidase Homologue D; MAPK, mitogen-activated protein kinase; CDPK, $\mathrm{Ca}^{2+}$-dependent protein kinase; Cf-4, C. fulvum resistance gene mediating recognition of Avr4; SOBIR1, Suppressor Of BIR1 - 1; RLCK, receptor-like cytoplasmic kinase; Avr4, Avirulence 4; WRKYs, WRKY transcription factors.

\section{Constitutive immunity induced by AtSOBIR1 as a tool to study the immune signalling pathway}

SOBIR1 is a positive regulator of defence, and plays an essential role in numerous immune pathways. The overexpression and mis-regulation of defence regulators may lead to spontaneous activation of immune responses, which was shown for SOBIR1 in Chapter 2.

Interestingly, constitutive immune activity was found for AtSOBIR1, but not for its homologues from tomato or $N$. benthamiana. We performed SOBIR1 overexpression in $N$. benthamiana and tobacco, so we argue that likely endogenous phosphatases from this Solanaceous plant are able to keep Solanaceous versions of SOBIR1 in check by negatively regulating its kinase domain (KD) through de-phosphorylation (Fig. 2). However, these Solanaceous phosphatases might not have enough affinity for AtSOBIR1 to efficiently keep its KD in check, leading to constitutive activity and eventually cell death. In a suppressor screen in Arabidopsis, AtSOBIR1 was already found to be a positive regulator of defence, and was shown to induce cell death upon its overexpression in Arabidopsis as well (Gao et al., 2009). So, maybe some intrinsic differences between SOBIR1 from Solanaceous plants and SOBIR1 from Arabidopsis add to cause the constitutive immune phenotype. Future overexpression experiments with chimeric proteins combining parts of Arabidopsis and tomato SOBIR1 might shed light on key sequences required for constitutive immune activity. Additionally, heterologous over-expression experiments of SOBIR1 homologues covering diverse plant species from Brassicaceae to Solanaceae might show the conservation of the property of the SOBIR1 homologues to induce constitutive immunity.

The constitutive immune response, visible as cell death, initiated by the overexpression of AtSOBIR1 in tobacco and $N$. benthamiana was shown to be a useful tool in many experiments, and will remain so in dissecting the various downstream components of the signalling cascade leading to the HR.

\section{SOBIR1 is a central target for effectors to suppress immunity}

SOBIR1 is a central regulator of defence (Liebrand et al., 2014). Probably, plants have evolved SOBIR1 as an RLP-docking station in order to facilitate the evolution of novel 
RLPs to recognize additional ExIPs of pathogens. In tomato, for example, the Cf genes are present in clusters of homologues, thereby promoting sequence exchange by unequal crossing over during meiosis (Parniske et al., 1997; Andolfo et al., 2013). In this way, only the DNA sequences encoding RLPs need to be duplicated, recombined, and diversified, and not the sequences encoding complete receptors carrying intracellular KDs, which would potentially give rise to recombinants that have lost their kinase activity and have become non-functional. Furthermore, only new RLPS that are still able to properly interact with SOBIR1 will be retained in the population. These advantages might explain why the system of bimolecular RLKs, consisting of ExIP-detecting RLPs interacting with SOBIR1, has been retained during evolution in parallel with RLKs recognising ExIPs and carrying their own integrated KD.

The centrality of SOBIR1 makes it a logical target for effectors from pathogens with the aim to suppress defence responses (Chapter 2). In view of the evolutionary arms race between host plants and pathogens, one could argue that it would be logical for SOBIR1 to evolve to evade targeting by effectors. However, the sequence of SOBIR1 is very conserved in the Plant Kingdom (Liebrand et al., 2014). Probably, conservation of the sequence of SOBIR1 is crucial, as it plays a role in many processes, and too much variation is detrimental. Other mechanisms might have evolved to protect SOBIR1 from being targeted, like a guard that monitors any changes of SOBIR1.

\section{SOBIR1 and BAK1, a transphosphorylating couple}

Transphosphorylation of signalling partners is an important molecular switch in various cellular processes, including plant defence. After BAK1 recruitment to RLKs that are activated by their ligand, transphosphorylation between the KDs of the RLK and BAK1 has been shown to initiate downstream defence responses (Lu et al., 2010; Schwessinger et al., 2011; Yan et al., 2012). In Chapter 3 it is shown that also transphosphorylation between SOBIR1 and BAK1 takes place, and that this process is essential to initiate defence responses downstream of the RLP Cf-4 (Fig. 2).

Based on our finding, and the findings of others working on the elucidation of similar signalling pathways, we propose that SOBIR1 trans-autophosphorylation takes place first. Upon BAK1 recruitment, which is facilitated either by the altered phosphorylation state of the SOBIR1-KD and/or Avr4-binding to the LRRs of Cf4, phosphorylation of BAK1 by SOBIR1 likely takes place, which locks BAK1 in an activated state. BAK1 then in its turn phosphorylates SOBIR1 (Chapter 3). Additionally, specific phosphorylation sites of SOBIR1 that are essential for immune signalling were determined (Chapter 4). AvrPto did not affect the interaction between Cf-4 and SOBIR1, and also not the recruitment of BAK1 upon activation of the Cf-4/SOBIR1 complex by Avr4. Possibly, suppression of SOBIR1-mediated immunity by AvrPto is caused by dephosphorylation of SOBIR1, but this remains to be elucidated. 
To stay as close as possible to a natural situation, the studies presented here were performed in planta. This does come with the drawback that the participation of endogenous proteins in various signalling-related processes cannot be excluded. Additionally, the protein purification methods that were used appeared to have their limitations, and no differential phosphorylation of SOBIR1 downstream of Cf-4 could be determined. Future experiments, in which the kinase domains of SOBIR1 and BAK1, including wild-type as well as mutant variants (kinase-dead, phospho-dead, and phospho-mimic variants), are produced in bacterial cells in different combinations and tested for their phosphorylation pattern, would be helpful to substantiate the proposed models. Although one should be aware that artefacts could occur when expressing plant proteins in such an artificial system, these complementary experiments could potentially further support the conclusions that are drawn from the in planta experiments.

Specific differential phosphorylation of SOBIR 1 is hypothesised to activate specific downstream responses, and differential affinity to signalling partners is proposed to play a role in this. Possibly, phosphorylation of several of the important residues identified in Chapter 4 affects the recruitment of BAK1, and/or the interaction with an RLCK. Co-immunoprecipitation (co-IP) experiments in $N$. benthamiana to test these hypotheses are challenging, as endogenous wild-type SOBIR1 is always present, even upon virus-induced gene silencing (VIGS) of NbSOBIR1(-like). The fast development of new techniques to knockout genes, like the CRISPR-Cas technology, should enable future experiments to test whether the individual phospho-dead mutants of SOBIR1 can still recruit BAK1 and/or interact with potentially involved RLCKs.

\section{The future of the C. fulvum - tomato pathosystem}

For many years, the Cladosporium fulvum - tomato pathosystem has been a key model system that has enabled scientists to study the molecular aspects of plantmicrobe interactions in detail (Joosten \& de Wit, 1999; Stergiopoulos \& de Wit, 2009; de Wit, 2016). Many plant-pathogen interaction studies are based on the model plant Arabidopsis, which is very useful to acquire fundamental knowledge. However, it is advantageous to study the plant immune system in Solanaceous plants, to be one step closer to possible applications in Solanaceous crop plants like tomato, potato, and pepper.

In the many years of research focussing on Solanaceous plants like tomato and $N$. benthamiana, a highly versatile toolbox has been developed to study plant-pathogen interactions, including tools like VIGS, transient and stable transformation, co-IP, etc.. In this thesis, many of these tools were used.

Although this toolbox facilitates a diverse range of experiments, several drawbacks could not yet be circumvented. For instance, overexpression might cause artefacts. In 
natural situations, gene expression is regulated in such a way that the location where the protein accumulates is strictly defined and the amount of protein that is produced is tightly regulated. When overexpression is performed, proteins might accumulate at subcellular locations where they normally are not present. However, by including the correct controls it is nevertheless reasonable to perform overexpression experiments the way they were done in this thesis.

Knocking down the expression of a gene by VIGS does not result in a complete knockout. As mentioned before, this might hamper testing the specific roles of particular proteins as residual amounts of the protein will always be present. In the near future, gene editing by CRISPR-Cas will enable the development of true knockouts. However, VIGS is a versatile tool, as for example a gene knockout can be lethal whereas a gene knockdown in most cases is not. Furthermore, a VIGS experiment can be designed to target several homologous genes at the same time. This can enable to study a set of genes which could not be studied easily using individual knockouts, due to redundancy. For example, the VIGS experiments that were performed to determine the role of RLCKs in the Cf-4/Avr4-triggered HR, did not lead to a conclusion (Chapter 5). Future experiments targeting sets of homologous RLCKs that possibly function redundantly, might decipher the role of Solanaceous RLCKs downstream of the Cf-4/SOBIR1 complex.

The results of the experiments described in this thesis shed light on the molecular basis of RLP/SOBIR1-mediated defence. However, no real pathogen inoculation assays were performed. The assumption is made that for instance HR and MAPK activation represent a successful defence response (Lam et al., 2001; Greenberg \& Yao, 2004; Lee et al., 2015). Various groups have published about the role of RLPs and SOBIR1 in defence against numerous pathogens (Chapter 1). The current work was aimed to zoom in to the molecular basis of RLP/SOBIR1-triggered signalling. Obviously, it would be very interesting to test the effect of, for instance, differential phosphorylation of SOBIR1 on its role in C. fulvum resistance, and resistance to other pathogens. As the results of pathogen assays are often not black and white, but highly variable, it will probably be very challenging to test the role of differential phosphorylation of SOBIR1 in pathogen assays.

Some progress can be made concerning the way immune response screenings are perform. In this thesis, the HR was often used as a visual score. Recent technical advances have made it possible to screen for cell death by detecting autofluorescence of the dying cells, which enables quantification. Furthermore, next to observing the $H R$, the production of ROS can also be used as a readout for defence responses. These measurements are performed using detached leaves, which makes it impossible to observe the response again at a later timepoint. Nevertheless, future experiments will benefit from such more exact quantification of the intensity of the defence response. 


\section{Conclusions}

Deciphering the molecular mechanisms behind plant immunity is important, because a profound knowledge on plant resistance mechanisms will help to improve broad spectrum plant resistance in a sustainable fashion, and it will improve our agricultural practices to secure food production.

This thesis contributes to deepen our knowledge on the plant defence response against pathogens. Several signalling steps that play a role in the RLP/SOBIR1/BAK1 complex have been clarified. This additional knowledge about the mode of action of SOBIR1, which plays a broad role in plant immunity, will be a basis for future research on RLPs. Plant genomes contain numerous genes encoding RLPs, and for most of them the function is still not known. Discovery of the roles of the different RLPs might help plant breeders in their efforts to breed for resistant crops and will eventually allow growers to eliminate the use of chemicals to prevent crop losses by diseases.

The publication of the zigzag model in 2006 was a revolution in the field of molecular phytopathology (Jones \& Dangl, 2006). Due to recent advances in molecular research, of which the results challenge the zigzag model, the invasion model was proposed a few years ago (Cook et al., 2015). With the knowledge of today, an update of the invasion model is presented in this thesis, which differentiates between extracellularly- and intracellularly-triggered defence. This spatial invasion model will facilitate the scientists working in the field of molecular phytopathology to express their findings in a clear way. 


\section{References}

AbuQamar S, Chai M-F, Luo H, Song F, Mengiste T. 2008. Tomato protein kinase $1 \mathrm{~b}$ mediates signaling of plant responses to necrotrophic fungi and insect herbivory. The Plant Cell 20: 1964-1983.

Albert I, Böhm H, Albert M, Feiler CE, Imkampe J, Wallmeroth N, Brancato C, Raaymakers TM, Oome S, Zhang H, Krol E, Grefen C, Gust AA, Chai J, Hedrich R, Van den Ackerveken G, Nürnberger T. 2015. An RLP23-SOBIR1-BAK1 complex mediates NLP-triggered immunity. Nature Plants 1: doi:10.1038/nplants.2015.1140.

Andolfo G, Sanseverino W, Rombauts S, Peer Y, Bradeen J, Carputo D, Frusciante L, Ercolano M. 2013. Overview of tomato (Solanum lycopersicum) candidate pathogen recognition genes reveals important Solanum R locus dynamics. New Phytologist 197: 223-237.

Bar M, Sharfman M, Ron M, Avni A. 2010. BAK1 is required for the attenuation of ethylene-inducing xylanase (Eix)-induced defense responses by the decoy receptor LeEix1. The Plant Journal 63: 791 800.

Ben Khaled S, Postma J, Robatzek S. 2015. A moving view: subcellular trafficking processes in pattern recognition receptor-triggered plant immunity. Annual Review of Phytopathology 53: 379-402.

Bendahmane A, Kanyuka K, Baulcombe DC. 1999. The $R x$ gene from potato controls separate virus resistance and cell death responses. The Plant Cell 11: 781-791.

Bi G, Liebrand TW, Cordewener JH, America AH, Xu X, Joosten MHAJ. 2014. Arabidopsis thaliana receptor-like protein AtRLP23 associates with the receptor-like kinase AtSOBIR1. Plant Signaling \& Behavior 9: e27937.

Böhm H, Albert I, Oome S, Raaymakers TM, Van den Ackerveken G, Nürnberger T. 2014. A conserved peptide pattern from a widespread microbial virulence factor triggers pattern-induced immunity in Arabidopsis. PLOS Pathogens 10: e1004491.

Boller T, Felix G. 2009. A renaissance of elicitors: perception of microbe-associated molecular patterns and danger signals by pattern-recognition receptors. Annual Review of Plant Biology 60: 379-406.

Boller T, He SY. 2009. Innate immunity in plants: an arms race between pattern recognition receptors in plants and effectors in microbial pathogens. Science 324: 742-744.

Bücherl CA, Jarsch IK, Schudoma C, Segonzac C, Mbengue M, Robatzek S, MacLean D, Ott T, Zipfel C. 2017. Plant immune and growth receptors share common signalling components but localise to distinct plasma membrane nanodomains. elife 6: e25114.

Catanzariti AM, Do HT, Bru P, Sain M, Thatcher LF, Rep M, Jones DA. 2017. The tomato I gene for Fusarium wilt resistance encodes an atypical leucine-rich repeat receptor-like protein whose function is nevertheless dependent on SOBIR1 and SERK3/BAK1. The Plant Journal 89: 1195-1209.

Chaparro-Garcia A, Wilkinson RC, Gimenez-Ibanez S, Findlay K, Coffey MD, Zipfel C, Rathjen JP, Kamoun S, Schornack S. 2011. The receptor-like kinase SERK3/BAK1 is required for basal resistance against the late blight pathogen Phytophthora infestans in Nicotiana benthamiana. PLOS ONE 6: e16608.

Chen X, Zuo S, Schwessinger B, Chern M, Canlas PE, Ruan D, Zhou X, Wang J, Daudi A, Petzold CJ. 2014. An XA21-associated kinase (OsSERK2) regulates immunity mediated by the XA21 and XA3 immune receptors. Molecular Plant 7: 874-892.

Chinchilla D, Shan L, He P, de Vries S, Kemmerling B. 2009. One for all: the receptor-associated kinase BAK1. Trends in Plant Science 14: 535-541.

Chinchilla D, Zipfel C, Robatzek S, Kemmerling B, Nürnberger T, Jones JD, Felix G, Boller T. 2007. A flagellin-induced complex of the receptor FLS2 and BAK1 initiates plant defence. Nature 448: 497500.

Cook DE, Mesarich CH, Thomma BP. 2015. Understanding plant immunity as a surveillance system to detect invasion. Annual Review of Phytopathology 53: 541-563. 
Couto $D$, Niebergall $R$, Liang $X$, Bücherl CA, Sklenar J, Macho AP, Ntoukakis V, Derbyshire $P$, Altenbach D, Maclean D, Robatzek S, Uhrig J, Menke F, Zhou J-M, Zipfel C. 2016. The Arabidopsis protein phosphatase PP2C38 negatively regulates the central immune kinase BIK1. PLOS Pathogens 12: e1005811.

Couto D, Zipfel C. 2016. Regulation of pattern recognition receptor signalling in plants. Nature Reviews Immunology 16: 537-552.

De Jonge R, van Esse HP, Kombrink A, Shinya T, Desaki Y, Bours R, van der Krol S, Shibuya N, Joosten MHAJ, Thomma BP. 2010. Conserved fungal LysM effector Ecp6 prevents chitin-triggered immunity in plants. Science 329: 953-955.

De Wit PJ. 1995. Fungal avirulence genes and plant resistance genes: unraveling the molecular basis of gene-for-gene interactions. Advances in Botanical Research 21: 147-185.

De Wit, P.J.G.M. 2016. Cladosporium fulvum effectors: weapons in the arms race with tomato. Annual Review of Phytopathology 54: 1-23

De Wit PJ, Mehrabi R, van den Burg HA, Stergiopoulos I. 2009. Fungal effector proteins: past, present and future. Molecular Plant Pathology 10: 735-747.

Dodds PN, Rathjen JP. 2010. Plant immunity: towards an integrated view of plant-pathogen interactions. Nature Reviews Genetics 11: 539-548.

Doehlemann G, Hemetsberger C. 2013. Apoplastic immunity and its suppression by filamentous plant pathogens. New Phytologist 198: 1001-1016.

Domazakis E, Wouters D, Visser R, Kamoun S, Joosten MHAJ, Vleeshouwers VGAA. 2018. The ELR-SOBIR1 complex functions as a two-component RLK to mount defense against Phytophthora infestans. Molecular Plant-Microbe Interactions. 10.1094/mpmi-09-17-0217-r

Du J, Verzaux E, Chaparro-Garcia A, Bijsterbosch G, Keizer LP, Zhou J, Liebrand TW, Xie C, Govers F, Robatzek S. 2015. Elicitin recognition confers enhanced resistance to Phytophthora infestans in potato. Nature Plants 1: 15034.

Eckardt NA. 2011. BIK1 function in plant growth and defense signaling. The Plant Cell 23: 2806-2806.

Etalo DW, Stulemeijer IJE, Peter van Esse H, de Vos RCH, Bouwmeester HJ, Joosten MHAJ. 2013. System-Wide hypersensitive response-associated transcriptome and metabolome reprogramming in tomato. Plant Physiology 162: 1599-1617.

Flor H. 1942. Inheritance of pathogenicity in Melampsora lini. Phytopathology 32: 653-669.

Flor HH. 1971. Current status of the gene-for-gene concept. Annual Review of Phytopathology 9: 275-296.

Fradin EF, Abd-EI-Haliem A, Masini L, van den Berg GCM, Joosten MHAJ, Thomma BPHJ. 2011. Interfamily transfer of tomato ve1 mediates Verticillium resistance in Arabidopsis. Plant Physiology 156: 2255-2265.

Fradin EF, Zhang Z, Ayala JCJ, Castroverde CDM, Nazar RN, Robb J, Liu CM, Thomma BPHJ. 2009. Genetic dissection of Verticillium wilt resistance mediated by tomato Ve1. Plant Physiology 150: 320-332.

Frescatada-Rosa M, Robatzek S, Kuhn H. 2015. Should I stay or should I go? Traffic control for plant pattern recognition receptors. Current Opinion in Plant Biology 28: 23-29.

Gao M, Wang X, Wang D, Xu F, Ding X, Zhang Z, Bi D, Cheng YT, Chen S, Li X, Zhang Y. 2009. Regulation of cell death and innate immunity by two receptor-like kinases in Arabidopsis. Cell Host and Microbe 6: 34-44.

Gómez-Gómez L, Boller T. 2000. FLS2: an LRR receptor-like kinase involved in the perception of the bacterial elicitor flagellin in Arabidopsis. Molecular Cell 5: 1003-1011.

Greenberg JT, Yao N. 2004. The role and regulation of programmed cell death in plant-pathogen interactions. Cellular Microbiology 6: 201-211.

Gross BL, Olsen KM. 2010. Genetic perspectives on crop domestication. Trends in Plant Science 15: 529537.

Gust AA, Felix G. 2014. Receptor like proteins associate with SOBIR1-type of adaptors to form bimolecular receptor kinases. Current Opinion in Plant Biology 21: 104-111. 
Halter T, Imkampe J, Mazzotta S, Wierzba M, Postel S, Bücherl C, Kiefer C, Stahl M, Chinchilla D, Wang X, Nürnberger T, Zipfel C, Clouse S, Borst Jan W, Boeren S, de Vries Sacco C, Tax F, Kemmerling B. 2014. The leucine-rich repeat receptor kinase BIR2 is a negative regulator of bAK1 in plant immunity. Current Biology 24: 134-143.

Heath MC. 1981. A generalized concept of host-parasite specificity. Phytopathology 71: 1121-1123.

Heese A, Hann DR, Gimenez-Ibanez S, Jones AME, He K, Li J, Schroeder JI, Peck SC, Rathjen JP. 2007. The receptor-like kinase SERK3/BAK1 is a central regulator of innate immunity in plants. Proceedings of the National Academy of Sciences of the United States of America 104: 12217-12222.

Hegenauer V, Fürst U, Kaiser B, Smoker M, Zipfel C, Felix G, StahI M, Albert M. 2016. Detection of the plant parasite Cuscuta reflexa by a tomato cell surface receptor. Science 353: 478-481.

Hind SR, Strickler SR, Boyle PC, Dunham DM, Bao Z, O'Doherty IM, Baccile JA, Hoki JS, Viox EG, Clarke CR. 2016. Tomato receptor FLAGELLIN-SENSING 3 binds flgll-28 and activates the plant immune system. Nature Plants 2: 16128.

Hohmann U, Lau K, Hothorn M. 2017. The structural basis of ligand perception and signal activation by receptor kinases. Annual Review of Plant Biology 68: 109-137.

Hohmann U, Santiago J, Nicolet J, Olsson V, Spiga FM, Hothorn LA, Butenko MA, Hothorn M. 2018. Mechanistic basis for the activation of plant membrane receptor kinases by SERK-family coreceptors. Proceedings of the National Academy of Sciences of the United States of America. 10.1073/pnas.1714972115

Holton N, Nekrasov V, Ronald PC, Zipfel C. 2015. The phylogenetically-related pattern recognition receptors EFR and XA21 recruit similar immune signaling components in monocots and dicots. PLOS Pathogens 11: e1004602.

Huffaker A, Pearce G, Ryan CA. 2006. An endogenous peptide signal in Arabidopsis activates components of the innate immune response. Proceedings of the National Academy of Sciences of the United States of America 103: 10098-10103.

Imkampe J, Halter T, Huang S, Schulze S, Mazzotta S, Schmidt N, Manstretta R, Postel S, Wierzba M, Yang Y, vanDongen WM, Stahl M, Zipfel C, Goshe MB, Clouse S, de Vries SC, Tax F, Wang X, Kemmerling B. 2017. The Arabidopsis leucine-rich repeat receptor kinase BIR3 negatively regulates BAK1 receptor complex formation and stabilizes BAK1. The Plant Cell 29: 2285-2303.

Jehle AK, Fürst U, Lipschis M, Albert M, Felix G. 2013a. Perception of the novel MAMP eMax from different Xanthomonas species requires the Arabidopsis receptor-like protein ReMAX and the receptor kinase SOBIR. Plant Signaling \& Behavior 8: e27408.

Jehle AK, Lipschis M, Albert M, Fallahzadeh-Mamaghani V, Fürst U, Mueller K, Felix G. 2013b. The receptor-like protein ReMAX of Arabidopsis detects the microbe-associated molecular pattern eMax from Xanthomonas. The Plant Cell 25: 2330-2340.

Jones JD, Dangl JL. 2006. The plant immune system. Nature 444: 323-329.

Joosten MHAJ, De Wit P. 1999. The tomato-Cladosporium fulvum interaction: a versatile experimental system to study plant-pathogen interactions. Annual Review of Phytopathology 37: 335-367.

Joosten MHAJ, Cozijnsen TJ, De Wit PJ. 1994. Host resistance to a fungal tomato pathogen lost by a single base-pair change in an avirulence gene. Nature 367: 384.

Kadota Y, Sklenar J, Derbyshire P, Stransfeld L, Asai S, Ntoukakis V, Jones Jonathan D, Shirasu K, Menke F, Jones A, Zipfel C. 2014. Direct regulation of the NADPH Oxidase RBOHD by the PRRassociated kinase BIK1 during plant immunity. Molecular Cell 54: 43-55.

Khan M, Seto D, Subramaniam R, Desveaux D. 2018. Oh, the places they'll go! A survey of phytopathogen effectors and their host targets. The Plant Journal 93: 651-663.

Kohler AC, Chen L-H, Hurlburt N, Salvucci A, Schwessinger B, Fisher AJ, Stergiopoulos I. 2016. Structural analysis of an Avr4 effector ortholog offers insight into chitin-binding and recognition by the Cf-4 receptor. The Plant Cell 28: 1945-1965

Kourelis J, van der Hoorn RAL. 2018. Defended to the nines: 25 years of resistance gene cloning identifies nine mechanisms for R protein function. The Plant Cell 30: 285-299. 
Lal NK, Nagalakshmi U, Hurlburt NK, Flores R, Bak A, Sone P, Ma X, Song G, Walley J, Shan L, He P, Casteel C, Fisher AJ, Dinesh-Kumar SP. 2018. The receptor-like cytoplasmic kinase BIK1 localizes to the nucleus and regulates defense hormone expression during plant innate immunity. Cell Host \& Microbe 23: 485-497.

Lam E, Kato N, Lawton M. 2001. Programmed cell death, mitochondria and the plant hypersensitive response. Nature 411: 848-853.

Larkan NJ, Ma L, Borhan MH. 2015. The Brassica napus receptor-like protein RLM2 is encoded by a second allele of the LepR3/RIm2 blackleg resistance locus. Plant Biotechnology Journal 13: 983-992.

Lee H-A, Lee H-Y, Seo E, Lee J, Kim S-B, Oh S, Choi E, Choi E, Lee SE, Choi D. 2017. Current understandings of plant nonhost resistance. Molecular Plant-Microbe Interactions 30: 5-15.

Lee J, Eschen-Lippold L, Lassowskat I, Böttcher C, Scheel D. 2015. Cellular reprogramming through mitogen-activated protein kinases. Frontiers in Plant Science 6: 940.

Li B, Meng X, Shan L, He P. 2016. Transcriptional regulation of pattern-triggered immunity in plants. Cell Host \& Microbe 19: 641-650.

Li J, Tax FE. 2013. Receptor-like kinases: key regulators of plant development and defense. Journal of Integrative Plant Biology 55: 1184-1187.

Li L, Li M, Yu L, Zhou Z, Liang X, Liu Z, Cai G, Gao L, Zhang X, Wang Y, Chen S, Zhou J-M. 2014. The FLS2-associated kinase BIK1 directly phosphorylates the NADPH oxidase RbohD to control plant immunity. Cell Host \& Microbe 15: 329-338.

Liang X, Zhou J-M. 2018. Receptor-like cytoplasmic kinases: central players in plant receptor kinasemediated signaling. Annual Review of Plant Biology 69: 267-299.

Liebrand TWH, van den Berg GCM, Zhang Z, Smit P, Cordewener JHG, America AHP, Sklenar J, Jones AME, Tameling WIL, Robatzek S, Thomma BPHJ, Joosten MHAJ. 2013. Receptor-like kinase SOBIR1/EVR interacts with receptor-like proteins in plant immunity against fungal infection. Proceedings of the National Academy of Sciences of the United States of America 110: 10010-10015.

Liebrand TWH, van den Burg HA, Joosten MHAJ. 2014. Two for all: receptor-associated kinases SOBIR1 and BAK1. Trends in Plant Science 19: 123-132.

Lin W, Li B, Lu D, Chen S, Zhu N, He P, Shan L. 2014. Tyrosine phosphorylation of protein kinase complex BAK1/BIK1 mediates Arabidopsis innate immunity. Proceedings of the National Academy of Sciences of the United States of America 111: 3632-3637.

Lin W, Lu D, Gao X, Jiang S, Ma X, Wang Z, Mengiste T, He P, Shan L. 2013. Inverse modulation of plant immune and brassinosteroid signaling pathways by the receptor-like cytoplasmic kinase BIK1. Proceedings of the National Academy of Sciences of the United States of America 110: 12114-12119.

Liu Z, Wu Y, Yang F, Zhang Y, Chen S, Xie Q, Tian X, Zhou J-M. 2013. BIK1 interacts with PEPRs to mediate ethylene-induced immunity. Proceedings of the National Academy of Sciences of the United States of America 110: 6205-6210.

Lu D, Lin W, Gao X, Wu S, Cheng C, Avila J, Heese A, Devarenne TP, He P, Shan L. 2011. Direct ubiquitination of pattern recognition receptor FLS2 attenuates plant innate immunity. Science $\mathbf{3 3 2}$ : 1439-1442.

Lu D, Wu S, Gao X, Zhang Y, Shan L, He P. 2010. A receptor-like cytoplasmic kinase, BIK1, associates with a flagellin receptor complex to initiate plant innate immunity. Proceedings of the National Academy of Sciences of the United States of America 107: 496-501.

Ma L, Borhan MH. 2015. The receptor-like kinase SOBIR1 interacts with Brassica napus LepR3 and is required for Leptosphaeria maculans AvrLm1-triggered immunity. Frontiers in Plant Science 6: 933.

Ma X, Xu G, He P, Shan L. 2016. SERKing Coreceptors for Receptors. Trends in Plant Science 21: 10171033.

Macho AP, Lozano-Durán R, Zipfel C. 2015. Importance of tyrosine phosphorylation in receptor kinase complexes. Trends in Plant Science 20: 269-272. 
Macho AP, Schwessinger B, Ntoukakis V, Brutus A, Segonzac C, Roy S, Kadota Y, Oh MH, Sklenar J, Derbyshire P, Lozano-Durán R, Gro Malinovsky F, Monaghan J, Menke FL, Huber SC, Yang He S, Zipfel C. 2014. A bacterial tyrosine phosphatase inhibits plant pattern recognition receptor activation. Science 343: 1509-1512.

Macho AP, Zipfel C. 2014. Plant PRRs and the activation of innate immune signaling. Molecular Cell 54: 263-272.

May MJ, Hammond-Kosack KE, Jones JD. 1996. Involvement of reactive oxygen species, glutathione metabolism, and lipid peroxidation in the Cf-gene-dependent defense response of tomato cotyledons induced by race-specific elicitors of Cladosporium fulvum. Plant Physiology 110: 13671379.

Mbengue M, Bourdais G, Gervasi F, Beck M, Zhou J, Spallek T, Bartels S, Boller T, Ueda T, Kuhn H, Robatzek S. 2016. Clathrin-dependent endocytosis is required for immunity mediated by pattern recognition receptor kinases. Proceedings of the National Academy of Sciences of the United States of America 113: 11034-11039.

Mesarich CH, Stergiopoulos I, Beenen HG, Cordovez V, Guo Y, Karimi Jashni M, Bradshaw RE, de Wit PJGM. 2015. A conserved proline residue in Dothideomycete Avr4 effector proteins is required to trigger a Cf-4-dependent hypersensitive response. Molecular Plant Pathology 17: 84-95.

Meyer RS, Purugganan MD. 2013. Evolution of crop species: genetics of domestication and diversification. Nature Reviews Genetics 14: 840.

Oh MH, Wu X, Clouse SD, Huber SC. 2011. Functional importance of BAK1 tyrosine phosphorylation in vivo. Plant Signaling \& Behavior 6: 400-405.

Park C-J, Peng Y, Chen X, Dardick C, Ruan D, Bart R, Canlas PE, Ronald PC. 2008. Rice XB15, a protein phosphatase $2 \mathrm{C}$, negatively regulates cell death and XA21-mediated innate immunity. PLoS Biology 6: e231.

Parniske M, Hammond-Kosack KE, Golstein C, Thomas CM, Jones DA, Harrison K, Wulff BB, Jones JD. 1997. Novel disease resistance specificities result from sequence exchange between tandemly repeated genes at the Cf-4/9 locus of tomato. Cell 91: 821-832.

Peng Y, van Wersch R, Zhang Y. 2018. Convergent and divergent signaling in PAMP-triggered immunity and effector-triggered immunity. Molecular Plant-Microbe Interactions 31: 403-409.

Piedras P, Hammond-Kosack KE, Harrison K, Jones JD. 1998. Rapid, Cf-9-and Avr9-dependent production of active oxygen species in tobacco suspension cultures. Molecular Plant-Microbe Interactions 11: 1155-1166.

Pieterse CM, Van der Does D, Zamioudis C, Leon-Reyes A, Van Wees SC. 2012. Hormonal modulation of plant immunity. Annual Review of Cell and Developmental Biology 28: 489-521.

Postel S, Küfner I, Beuter C, Mazzotta S, Schwedt A, Borlotti A, Halter T, Kemmerling B, Nürnberger T. 2010. The multifunctional leucine-rich repeat receptor kinase BAK1 is implicated in Arabidopsis development and immunity. European Journal of Cell Biology 89: 169-174.

Postma J, Liebrand TWH, Bi G, Evrard A, Bye RR, Mbengue M, Kuhn H, Joosten MHAJ, Robatzek SC. 2016. Avr4 promotes $\mathrm{Cf}-4$ receptor-like protein association with the BAK1/SERK3 receptor-like kinase to initiate receptor endocytosis and plant immunity. New Phytologist 210: 627-642.

Pruitt RN, Schwessinger B, Joe A, Thomas N, Liu F, Albert M, Robinson MR, Chan LJG, Luu DD, Chen H. 2015. The rice immune receptor XA21 recognizes a tyrosine-sulfated protein from a Gramnegative bacterium. Science Advances 1: e1500245.

Ranf S. 2017. Sensing of molecular patterns through cell surface immune receptors. Current Opinion in Plant Biology 38: 68-77.

Romeis T, Ludwig AA, Martin R, Jones JD. 2001. Calcium-dependent protein kinases play an essential role in a plant defence response. The EMBO Journal 20: 5556-5567.

Romeis T, Piedras $\mathbf{P}$, Jones JD. 2000. Resistance gene-dependent activation of a calcium-dependent protein kinase in the plant defense response. The Plant Cell 12: 803-815. 
Romeis T, Piedras P, Zhang S, Klessig DF, Hirt H, Jones JD. 1999. Rapid Avr9-and Cf-9-dependent activation of MAP kinases in tobacco cell cultures and leaves: convergence of resistance gene, elicitor, wound, and salicylate responses. The Plant Cell 11: 273-287.

Ron M, Avni A. 2004. The receptor for the fungal elicitor ethylene-inducing xylanase is a member of a resistance-like gene family in tomato. The Plant Cell 16: 1604-1615.

Rowland O, Ludwig AA, Merrick CJ, Baillieul F, Tracy FE, Durrant WE, Fritz-Laylin L, Nekrasov V, Sjölander K, Yoshioka H. 2005. Functional analysis of Avr9/Cf-9 rapidly elicited genes identifies a protein kinase, ACIK1, that is essential for full Cf-9-dependent disease resistance in tomato. The Plant Cell 17: 295-310.

Saur IM, Kadota Y, Sklenar J, Holton NJ, Smakowska E, Belkhadir Y, Zipfel C, Rathjen JP. 2016. $\mathrm{NbCSPR}$ underlies age-dependent immune responses to bacterial cold shock protein in Nicotiana benthamiana. Proceedings of the National Academy of Sciences of the United States of America 113: 3389-3394.

Schulze B, Mentzel T, Jehle AK, Mueller K, Beeler S, Boller T, Felix G, Chinchilla D. 2010. Rapid heteromerization and phosphorylation of ligand-activated plant transmembrane receptors and their associated kinase BAK1. Journal of Biological Chemistry 285: 9444-9451.

Schwessinger B, Roux M, Kadota Y, Ntoukakis V, Sklenar J, Jones A, Zipfel C. 2011. Phosphorylationdependent differential regulation of plant growth, cell death, and innate immunity by the regulatory receptor-like kinase BAK1. PLoS Genetics 7: e1002046-e1002046.

Segonzac C, Macho AP, Sanmartín M, Ntoukakis V, Sánchez-Serrano JJ, Zipfel C. 2014. Negative control of BAK1 by protein phosphatase 2A during plant innate immunity. The EMBO Journal 33 : 2069-2079.

Seybold H, Trempel F, Ranf S, Scheel D, Romeis T, Lee J. 2014. Ca2+ signalling in plant immune response: from pattern recognition receptors to $\mathrm{Ca} 2+$ decoding mechanisms. New Phytologist 204: 782-790.

Stam R, Mantelin S, McLellan H, Thilliez G. 2014. The role of effectors in nonhost resistance to filamentous plant pathogens. Frontiers in Plant Science 5: 582.

Stergiopoulos I, De Wit PJGM. 2009. Fungal effector proteins. Annual Review of Phytopathology 47: 233-263.

Stergiopoulos I, van den Burg HA, Ökmen B, Beenen HG, van Liere S, Kema GH, de Wit PJ. 2010. Tomato $\mathrm{Cf}$ resistance proteins mediate recognition of cognate homologous effectors from fungi pathogenic on dicots and monocots. Proceedings of the National Academy of Sciences of the United States of America 107: 7610-7615.

Stotz HU, Mitrousia GK, de Wit PJ, Fitt BD. 2014. Effector-triggered defence against apoplastic fungal pathogens. Trends in Plant Science 19: 491-500.

Stulemeijer IJ, Stratmann JW, Joosten MHAJ. 2007. Tomato mitogen-activated protein kinases LeMPK1, LeMPK2, and LeMPK3 are activated during the Cf-4/Avr4-induced hypersensitive response and have distinct phosphorylation specificities. Plant Physiology 144: 1481-1494.

Tang J, Han Z, Sun Y, Zhang H, Gong X, Chai J. 2015. Structural basis for recognition of an endogenous peptide by the plant receptor kinase PEPR1. Cell Research 25: 110.

Thomas CM, Jones DA, Parniske M, Harrison K, Balint-Kurti PJ, Hatzixanthis K, Jones JD. 1997. Characterization of the tomato $\mathrm{Cf}-4$ gene for resistance to Cladosporium fulvum identifies sequences that determine recognitional specificity in Cf-4 and Cf-9. The Plant Cell 9: 2209-2224.

Thomma BPHJ, Nürnberger T, Joosten MHAJ. 2011. Of PAMPs and effectors: the blurred PTI-ETI dichotomy. The Plant Cell 23: 4-15.

Van den Burg HA, Westerink N, Francoijs K-J, Roth R, Woestenenk E, Boeren S, de Wit PJ, Joosten MHAJ, Vervoort J. 2003. Natural disulfide bond-disrupted mutants of AVR4 of the tomato pathogen Cladosporium fulvum are sensitive to proteolysis, circumvent Cf-4-mediated resistance, but retain their chitin binding ability. Journal of Biological Chemistry 278: 27340-27346.

Van der Hoorn RA, Kamoun S. 2008. From guard to decoy: a new model for perception of plant pathogen effectors. The Plant Cell 20: 2009-2017. 
Van der Hoorn RA, Van der Ploeg A, de Wit PJ, Joosten MHAJ. 2001. The C-terminal dilysine motif for targeting to the endoplasmic reticulum is not required for Cf-9 function. Molecular Plant-Microbe Interactions 14: 412-415.

Veronese P, Nakagami H, Bluhm B, AbuQamar S, Chen X, Salmeron J, Dietrich RA, Hirt H, Mengiste T. 2006. The membrane-anchored BOTRYTIS-INDUCED KINASE1 plays distinct roles in Arabidopsis resistance to necrotrophic and biotrophic pathogens. The Plant Cell 18: 257-273.

Wan W-L. 2017. Comparative analysis of signaling pathways triggered by different pattern-recognition receptor-types. PhD thesis, University of Tübingen.

Wang G, Fiers M, Ellendorff U, Wang Z, de Wit PJ, Angenent GC, Thomma BP. 2010. The diverse roles of extracellular leucine-rich repeat-containing receptor-like proteins in plants. Critical Reviews in Plant Science 29: 285-299.

Wang L, Albert M, Einig E, Fürst U, Krust D, Felix G. 2016. The pattern-recognition receptor CORE of Solanaceae detects bacterial cold-shock protein. Nature Plants 2: 16185.

Wang X, Kota U, He K, Blackburn K, Li J, Goshe MB, Huber SC, Clouse SD. 2008. Sequential transphosphorylation of the BRI1/BAK1 receptor kinase complex impacts early events in brassinosteroid signaling. Developmental Cell 15: 220-235.

Wang Y, Xu Y, Sun Y, Wang H, Qi J, Wan B, Ye W, Lin Y, Shao Y, Dong S, Tyler BM, Wang Y. 2018. Leucine-rich repeat receptor-like gene screen reveals that Nicotiana RXEG1 regulates glycoside hydrolase 12 MAMP detection. Nature Communications 9: 594.

Yan H, Zhao Y, Shi H, Li J, Wang Y, Tang D. 2018. BRASSINOSTEROID-SIGNALING KINASE1 phosphorylates MAPKKK5 to regulate immunity in Arabidopsis. Plant Physiology 176: 2991-3002.

Yan L, Ma Y, Liu D, Wei X, Sun Y, Chen X, Zhao H, Zhou J, Wang Z, Shui W, Lou Z. 2012. Structural basis for the impact of phosphorylation on the activation of plant receptor-like kinase BAK1. Cell Research 22: 1304-1308.

Yasuda S, Okada K, Saijo Y. 2017. A look at plant immunity through the window of the multitasking coreceptor BAK1. Current Opinion in Plant Biology 38: 10-18.

Zhang L, Kars I, Essenstam B, Liebrand TW, Wagemakers L, Elberse J, Tagkalaki P, Tjoitang D, van den Ackerveken G, van Kan JA. 2014. Fungal endopolygalacturonases are recognized as microbeassociated molecular patterns by the Arabidopsis receptor-like protein RESPONSIVENESS TO BOTRYTIS POLYGALACTURONASES1. Plant Physiology 164: 352-364.

Zhang W, Fraiture M, Kolb D, Löffelhardt B, Desaki Y, Boutrot FFG, Tör M, Zipfel C, Gust AA, Brunner F. 2013. Arabidopsis receptor-like protein 30 and receptor-like kinase Suppressor Of BIR11/EVERSHED mediate innate immunity to necrotrophic fungi. The Plant Cell 25: 4227-4241.

Zhang X, Dodds PN, Bernoux M. 2017. What do we know about NOD-like receptors in plant immunity? Annual Review of Phytopathology 55: 205-229.

Zipfel C. 2014. Plant pattern-recognition receptors. Trends in Immunology 35: 345-351.

Zipfel C, Kunze G, Chinchilla D, Caniard A, Jones JD, Boller T, Felix G. 2006. Perception of the bacterial PAMP EF-Tu by the receptor EFR restricts Agrobacterium-mediated transformation. Cell 125: 749760.

Zipfel C, Oldroyd GED. 2017. Plant signalling in symbiosis and immunity. Nature 543: 328-336. 


\section{Summary}

We can learn from nature, by studying the mechanisms by which plants defend themselves against pathogens. This knowledge can be applied to improve crops in a sustainable fashion. Plants are sessile organisms with multiple layers of defence against pathogens. A prominent first layer of defence, which is similar to the innate immune system of mammalian cells, is provided by transmembrane (TM)receptors, which are present at the plant cell surface. Upon pathogen invasion of the extracellular (apoplastic) space, TM-receptors mediate the recognition of pathogenand/or host derived invasion patterns (IPs) in the extracellular space using their extracellular domain (ECD), which often consists of leucine-rich repeats (LRRs). Next to the ECD, TM-receptors have a single pass TM domain, and an intracellular domain. TM-receptors can be receptor-like kinases (LRR-RLKs), which contain an intracellular kinase domain to enable cytoplasmic signalling, or receptor-like proteins (LRRRLPs), which contain only a small cytoplasmic tail and no signalling domain. LRRRLKs and LRR-RLPs are here further referred to as RLKs and RLPs. The regulatory RLK Suppressor Of BIR1-1/Evershed (SOBIR1/EVR, further referred to as SOBIR1) was recently found to constitutively interact with RLPs, and thereby support RLP accumulation as a kind of scaffold protein. Additionally, SOBIR1 was proposed to provide a signalling domain to RLPs, to result in bimolecular RLKs. RLKs, as well as bimolecular RLP/SOBIR1 complexes, both recruit the co-receptor BrassinosteroidInsensitive 1 - Associated Kinase 1/Somatic Embryogenesis Receptor Kinase 3 (BAK1/ SERK3, further referred to as BAK1) upon ligand recognition by the primary ligand receptor. This BAK1 recruitment is thought to activate the TM-receptor complex for downstream signalling.

Cultivated tomato (Solanum lycopersicum, Sl) possesses cell surface receptors, introgressed from wild tomato varieties, that provide resistance to the biotrophic leaf pathogen Cladosporium fulvum. These so-called Cf proteins mediate recognition of secreted effectors (also known as avirulence factors (Avrs)) of the pathogen in the apoplast. These $\mathrm{Cf}$ proteins are RLPs, and have recently been shown to constitutively interact with the RLK SOBIR1. The work in this thesis was initiated to elucidate the nature of the signalling steps that take place downstream of RLP/SOBIR1 bimolecular RLKs. To this aim, the signalling events that take place upon activation of the Cf-4/ SOBIR1 complex by the matching C. fulvum effector Avr4 were studied.

In Chapter 1, the plant innate immune system is introduced, and an overview of the current knowledge is given. The main focus lays on cell surface receptor complexes with an ECD consisting of LRRs, on their regulation, and on the immune responses that they trigger. Moreover, the tomato $-C$. fulvum pathosystem is introduced, with emphasis on the Cf-4/Avr4 gene-for-gene pair. 
SOBIR1 is required for RLP-mediated resistance to a wide range of pathogens, and it is hypothesized that SOBIR1 is targeted by effector proteins of pathogens to suppress host defence responses. In Chapter $\mathbf{2}$ it is shown that AvrPto, an effector of the bacterial pathogen Pseudomonas syringae pv. tomato DC3000, interacts with SOBIR1 from Arabidopsis thaliana (At, further referred to as Arabidopsis) and with various Solanaceous SOBIR1 orthologues. This interaction is independent of SOBIR1 kinase activity. Interestingly, AvrPto suppresses AtSOBIR1-induced constitutive immunity, which is observed as cell death (the hypersensitive response (HR)) upon overexpression of AtSOBIR1 in N. benthamiana and tobacco. Additionally, AvrPto compromises the Avr4-triggered HR in Cf-4-transgenic $N$. benthamiana, without affecting Cf-4/SOBIR1/BAK1 complex formation. These results demonstrate that the bacterial effector AvrPto targets the regulatory RLK SOBIR1, and thereby compromises SOBIR1-mediated defence responses.

In Chapter $\mathbf{3}$ it is shown that kinase activity of SOBIR1 is not essential for its scaffold function, as the kinase-dead mutant SISOBIR10473N also stabilizes Cf4, similar to wild-type SOBIR1. However, kinase activity of SOBIR1 is crucial for downstream immune signalling, and therefore it is hypothesised that SOBIR1 transphosphorylates downstream signalling partners to initiate the activation of defence responses. Phosphorylation of signalling partners is an important molecular switch in various cellular processes, including plant defence. It was observed that AtSOBIR1, which constitutively activates immune responses upon its overexpression in $N$. benthamiana and tobacco, is highly phosphorylated in planta. Moreover, next to the required kinase activity of SOBIR1, kinase-active BAK1 is also essential for AtSOBIR1-induced constitutive immunity and for the phosphorylation of AtSOBIR1. Furthermore, the activation of a defence response upon perception of Avr4 by Cf- 4 depends on signalling-competent BAK1. These results, in addition to observations described in literature about other RLK signalling partners, suggest that SOBIR1 likely first transphosphorylates BAK1 upon its recruitment to the ligand-activated RLP/SOBIR1 complex, after which activated BAK1 transphosphorylates SOBIR1 to subsequently together initiate downstream signalling for immunity.

Phosphorylation of SOBIR1 appears to be important for its role in signalling for defence and Chapter 4 elaborates on the findings described in Chapter 3. In Chapter 4, amino acids of the kinase domain of SOBIR1 that could potentially be phosphorylated upon pathogen recognition are identified, and the role of these potential phosphorylation sites in signalling for defence is analysed. Mutational analyses and three-dimensional modelling showed that the threonine (Thr, T) residues T519, T523, and T529, which are all highly conserved in the activation segment of the kinase domain of SOBIR1, are important residues for the role of SOBIR1 in immune signalling. Phosphorylation of these sites likely locks SOBIR1 in an active conformation by controlling the conformation of the activation loop. 
Phosphorylation of these amino acids likely stimulates the interaction of T523 and T529 with the arginine (Arg, R) residue and the catalytic aspartic acid (Asp, D) residue of the 'RD' motif, respectively. Moreover, phosphorylation on T522, and the tyrosine (Tyr, Y) residues 532 and 538, which are also highly conserved, is likely generating substrate specificity and differential affinity for interacting partners. Coimmunoprecipitation of Cf-4-associated SOBIR1, through a pull-down of Cf-4 in the resting state and in the Avr4-activated state from $N$. benthamiana:Cf-4-eGFP plants, and subsequent analysis via mass spectrometry (MS), did not identify differential phosphorylation of the SOBIR1 kinase domain. However, in planta overexpression of AtSOBIR1-eGFP, followed by its immunoprecipitation and analysis via MS, revealed that AtSOBIR1 is phosphorylated on several serine (Ser, S) and Thr residues of its kinase domain, including T519. It is concluded that specific phosphorylation of the kinase domain of SOBIR1 likely enables this regulatory RLK to specifically switch on immune signalling downstream of RLPs.

Directly downstream of RLKs, plants employ receptor-like cytoplasmic kinases (RLCKs) to signal for defence. Botrytis-Induced Kinase 1 (BIK1) is a central RLCK that signals downstream of several RLKs, including Flagellin-Sensing 2 (FLS2) in Arabidopsis. In Chapter 5, BIK1 is shown to be also important for defence signalling downstream of RLP/SOBIR1-containing complexes, as AtBIK1 was found to interact with AtSOBIR1, as well as with SISOBIR1. Moreover, overexpression of the closest Solanaceous BIK1 orthologues enhanced the HR triggered by $\mathrm{Cf}-4$ upon recognition of Avr4. On the contrary, overexpression of AtBIK1 appeared to suppress the Cf-4/ Avr4-triggered HR. Although a silencing screen of a broad set of $N$. benthamiana BIK1-homologues did not point to a clear Solanaceous RLCK involved in the Cf-4/ SOBIR1-mediated HR, a split-luciferase assay showed the interaction between several tomato BIK1-homologues and SISOBIR1 and AtFLS2. Furthermore, the tomato RLCK Tomato Protein Kinase 1b (SITPK1b) was shown to specifically interact with SISOBIR1. Together, these data suggest that RLCKs play a role in signalling downstream of RLP/ SOBIR1 complexes.

TM-receptors need to be tightly controlled and regulated to ensure the triggering of a robust defence signal, and at the same time prevent false activation of defence responses. Physical separation of receptors and their co-receptors on the PM by negative regulators helps to keep signalling for defence in check, and thereby retain plant homeostasis concerning growth and development on the one hand and immunity on the other hand. The BAK1-Interacting RLK (BIR) protein family of Arabidopsis is a group of RLKs that negatively regulate immunity by interfering with the hetero-dimerization of the co-receptor BAK1 with ligand-binding immune-receptors. In Chapter 6, it is shown that the BIR protein family is conserved in Solanaceous plants. Gene silencing, overexpression, and protein-protein interaction studies show that the Solanaceous BIR1 orthologues are, similar to AtBIR1, negative regulators of 
cell death, and that BIR1 might suppress the Avr4-triggered HR in tomato containing $\mathrm{Cf}-4$. Additionally, BIR2 orthologues of tomato and $N$. benthamiana seem not to be involved in modulating the $\mathrm{Cf}-4$ /Avr4-triggered HR. From these results it is concluded that SIBIR1 might be a negative regulator of SOBIR1-mediated defence, possibly through its interaction with SIBAK1, whereas SIBIR2 appears not to be involved in the regulation of $\mathrm{Cf}-4 / \mathrm{SOBIR} 1$-mediated defence. This leads to the hypothesis that BIR1 and BIR2 each likely interact with a different pool of BAK1 present at the plasma membrane, and that each pool is probably involved in a different defence pathway.

Chapter $\mathbf{7}$ summarizes and discusses the major findings described in this thesis. The results of this thesis, together with other recent fundamental discoveries describing plant-microbe interactions on a molecular level, support a refinement of the invasion model that was developed to describe plant-microbe interactions. All TM-receptors with an LRR ECD recruit BAK1 upon their activation by the matching ligand, leading to transphosphorylation events and the initiation of downstream defence responses. These defence responses can be either mild or strong. Strikingly, there are RLP/SOBIR1 complexes that signal for a strong defence response including an $H R$, and also RLP/SOBIR1 complexes that signal for basal defence responses. All RLP/SOBIR1 complexes tested to far require BAK1 recruitment, and probably transphosphorylation of the kinase domains of SOBIR1 and BAK1 to initiate defence signalling. Therefore, as all signalling initiated by TM-receptors seems to be similar on the molecular level, this supports a spatial division rather than a division based on the intensity of the generated defence responses, which was up till now mostly used. In the 'spatial invasion model', IPs are therefore proposed to be classified as either extracellular IPs (ExIPs) or intracellular IPs (InIPs). As a consequence, recognition of ExIPs by TM-receptors leads to extracellularly-triggered immunity (ExTI), and recognition of InIPs by cytoplasmic receptors (which are mostly nucleotide-binding leucine-rich repeat receptors, NB-LRRs) leads to intracellularly-triggered immunity (InTI). Using this spatial dichotomy, the spatial invasion model facilitates a broadly including, but clearly distinguishing nomenclature to describe plant-microbe interactions. 


\section{Samenvatting}

Door te leren van de natuur en te onderzoeken hoe de verdediging van planten tegen ziekteverwekkers werkt, kan de vergaarde kennis worden ingezet om gewassen op een duurzame manier te verbeteren en te beschermen tegen ziektes. Planten hebben van nature meerdere verdedigingslagen waarmee ze zich kunnen verweren tegen ziekteverwekkers. Een prominente eerste laag, die overeenkomt met het aangeboren immuunsysteem van zoogdieren, bestaat uit transmembraan (TM)-receptoren die aanwezig zijn op het celoppervlak, het plasmamembraan (PM). Deze TM-receptoren kunnen ziekteverwekkers (pathogenen) die de extracellulaire (apoplastische) ruimtes van de bladeren van de plant binnendringen, herkennen. Hierbij worden zogenaamde 'invasiepatronen' (IPs), die vrijkomen tijdens de invasie door ziekteverwekkers, herkend door het extracellulaire deel van TM-receptoren. Het extracellulaire deel van TM-receptoren bestaat vaak uit een 'leucine-rich repeat' (LRR) domein. LRR domeinen zijn vaak betrokken bij eiwit-eiwit interacties. TM-receptoren kunnen 'receptor-like kinases' (RLKs) of 'receptor-like proteins' (RLPs) zijn. In dit proefschrift staan RLKs en RLPs met een extracellulair LRR domein centraal. Deze RLKs en RLPs zitten verankerd in het PM met een enkel TM domein. RLKs bezitten een intracellulair (cytoplasmatisch) kinase domein, dat ze in staat stelt om een signaal door te geven van de buitenkant naar de binnenkant van de cel. RLPs bezitten een dergelijk signaleringsdomein niet, maar hebben enkel een korte cytoplasmatische staart. Recentelijk is gevonden dat RLPs constitutief interacteren met de RLK 'Suppressor Of BIR 1-1/Evershed' (SOBIR1/EVR, verder SOBIR1 genoemd). SOBIR1 zorgt voor de stabilisatie van RLPs en is essentieel voor het functioneren van RLPs in de verdedigingsreacties tegen verschillende ziekteverwekkers. Een RLP en SOBIR1 vormen samen een tweeledige RLK. Wanneer een IP wordt herkend door een RLK of een RLP/SOBIR1 complex (wat dus een tweeledige RLK is), dan wordt de co-receptor 'Brassinosteroid-Insensitive 1 - Associated Kinase 1/Somatic Embryogenesis Receptor Kinase 3' (BAK1/SERK3, verder BAK1 genoemd) gerekruteerd naar de RLK of het RLP/ SOBIR1 complex. Deze associatie met BAK1 is nodig om het receptorcomplex te activeren en daarmee signalering naar het cytoplasma mogelijk te maken.

Gecultiveerde tomaat (Solanum lycopersicum, Sl) bezit verscheidene TMreceptoren die zorgen voor resistentie tegen de biotrofe schimmel Cladosporium fulvum, de veroorzakervan de Bladvlekkenziekte van tomaat. Deze resistentie eiwitten, 'Cfs' genoemd, zijn door veredelaars ingekruist vanuit wilde tomaat variëteiten. Deze $\mathrm{Cf}$ eiwitten zijn in staat om gesecreteerde effectoren (zogenaamde avirulentiefactoren (Avrs) van C. fulvum te herkennen. Cf eiwitten zijn RLPs en ze interacteren constitutief met SOBIR1. Het onderzoek dat is beschreven in dit proefschrift werd geïnitieerd om op te helderen hoe de signalering door RLPs, na de herkenning van extracellulaire IPs, werkt. Hiervoor is gebruik gemaakt van de RLP Cf-4, die constitutief met SOBIR1 
interacteert, en het effector eiwit Avr4 van C. fulvum dat specifiek wordt herkend door $\mathrm{Cf}-4$. Het onderzoek vond hoofdzakelijk plaats in planten van de nachtschadefamilie (Solanaceae), zodat de resultaten mogelijk tot verbetering van diverse gewassen uit deze familie, zoals tomaat, aardappel en paprika, zou kunnen leiden.

In Hoofdstuk 1 wordt de werking van het immuun systeem van planten geïntroduceerd. Er wordt een overzicht van wat hierover momenteel bekend is gegeven. Hierin staan TM-receptoren met een extracellulair LRR domein centraal. De regulatie van receptorcomplexen op de PM en de signaleringsroute van de PM tot in de celkern wordt besproken. Tot slot wordt in Hoofdstuk 1 het tomaat - C. fulvum pathosysteem beschreven, met de nadruk op het Cf-4/Avr4 gen-voor-gen koppel.

De regulerende RLK SOBIR1 is nodig voor RLP-gemedieerde resistentie tegen verscheidene ziekteverwekkers, zoals bacteriën, schimmels en oömyceten. Het lijkt dus een logische stap voor deze ziekteverwekkers om een centrale RLK zoals SOBIR1 als doelwit te kiezen en deze te manipuleren door middel van effector eiwitten, om op deze manier de verdedigingsreacties van de plant te onderdrukken. Hoofdstuk 2 laat zien dat het effector eiwit AvrPto van de bacteriële ziekteverwekker Pseudomonas syringae pv. tomato DC3000 interacteert met SOBIR1 van de modelplant Arabidopsis thaliana (At, verder Arabidopsis genoemd) en ook met de SOBIR1 orthologen van verscheidene planten uit de nachtschadefamilie. De interactie met AvrPto is onafhankelijk van de kinase-activiteit van SOBIR1. Overexpressie experimenten laten zien dat wanneer AtSOBIR1 tot overexpressie wordt gebracht in de tabakssoorten Nicotiana benthamiana $(\mathrm{Nb})$ en $N$. tabacum $(N t)$, er een constitutieve inductie van de resistentie reactie plaatsvindt, welke zichtbaar is als een lokale celdood (de zogenaamde 'overgevoeligheidsreactie' of 'hypersensitive response' (HR)). AvrPto onderdrukt deze celdood, wat waarschijnlijk een rol speelt bij de infectie van de plant door de bacterie. AvrPto kan ook de HR onderdrukken die geïnitieerd wordt door de herkenning van Avr4 door Cf-4 in Cf-4-transgene N. benthamiana planten. Deze onderdrukking door AvrPto vindt plaats zonder de vorming van Cf-4/SOBIR1/BAK1 complexen te beïnvloeden. Deze resultaten tonen aan dat de bacteriële effector AvrPto de RLK SOBIR1 als doelwit heeft om de resistentie reactie van de plant te onderdrukken.

SOBIR1 is een ondersteunend en regulerend eiwit voor RLPs, welke zelf geen kinase domein bezitten. SOBIR1 is essentieel voor de accumulatie van RLPs. In Hoofdstuk 3 wordt aangetoond dat deze ondersteunende functie onafhankelijk is van de kinase-activiteit van SOBIR1. De kinase-dode mutant SISOBIR1 $1^{\text {D473N }}$ is namelijk in staat om Cf-4 te stabiliseren, net als wild-type SOBIR1. Desalniettemin is de kinase-activiteit van SOBIR1 cruciaal voor de immuun signalering door Cf4. Het is dus zeer waarschijnlijk dat SOBIR1 een rol speelt in de signalering door partner eiwitten te transfosforyleren. Fosforylatie is een belangrijke moleculaire schakelaar in verschillende cellulaire processen, zo ook in de verdedigingsreactie 
tegen pathogenen. Uit de experimenten blijkt dat AtSOBIR1, welke een constitutieve activering van de HR geeft, duidelijk gefosforyleerd is in planta. Deze fosforylatiestatus blijkt afhankelijk van zijn eigen kinase-activiteit en ook van de kinase-activiteit van de co-receptor BAK1. Bovendien blijkt het vermogen van BAK1 om te signaleren essentieel voor de inductie van de constitutieve immuunreactie door AtSOBIR1, en ook voor de Cf-4/Avr4-geïnduceerde HR. Deze resultaten laten zien dat SOBIR1 waarschijnlijk eerst BAK1 transfosforyleert wanneer deze wordt gerekruteerd naar het $\mathrm{Cf}-4 / \mathrm{SOBIR} 1$ complex, waarna vervolgens de geactiveerde co-receptor BAK1 op zijn beurt SOBIR1 transfosforyleert. Deze transfosforylatie gebeurtenissen initiëren waarschijnlijk de cascade van vervolgstappen die uiteindelijk leidt tot een resistentie reactie.

Voortbordurend op de bevindingen die zijn beschreven in Hoofdstuk 3, wordt in Hoofdstuk 4 onderzocht welke aminozuren van het kinase domein (KD) van SOBIR1 mogelijk gefosforyleerd worden tijdens de immuun signalering en dus een rol spelen bij de signaleringsfunctie van SOBIR1. Mutatie analyses, in combinatie met drie dimensionale modellering van het KD van SOBIR1, laten zien dat de threonine ( $T h r, T)$ residuen T519, T523 en T529 essentiële aminozuur residuen zijn voor het functioneren van SOBIR1 in de immuun signalering. Deze Thr residuen bevinden zich in het activatie-segment van het KD van SOBIR1. Fosforylatie van deze aminozuur residuen is waarschijnlijk belangrijk om het activatiesegment van het KD van SOBIR1 in de actieve conformatie te brengen, onder andere door te interacteren met de residuen arginine (Arg, R) en asparaginezuur (Asp, D) (het 'RD' motief) van het katalytische domein. Er werd ook gevonden dat fosforylatie van T522 en van de tyrosine (Tyr, Y) residuen 532 en 538 een rol zou kunnen spelen in de specificiteit van het KD voor de binding van een substraat eiwit en in de affiniteit van het KD voor interacterende signaleringspartners. De co-immunoprecipitatie van Cf-4-geassocieerd SOBIR1, via de opzuivering van het fusie-eiwit Cf-4-eGFP, in de rusttoestand en in de door Avr4geactiveerde toestand, uit bladeren van $N$. benthamiana:Cf-4-eGFP planten, gevolgd door massa spectrometrie (MS), heeft niet geleid tot de identificatie van differentiëel gefosforyleerde aminozuren van SOBIR1. Directe immunoprecipitatie van het tot overexpressie gebrachte fusie-eiwit van AtSOBIR1-eGFP uit N. benthamiana, gevolgd door MS, laat zien dat AtSOBIR1 is gefosforyleerd op verscheidene serine (Ser, S) en Thr residuen. Samen genomen laten deze resultaten zien dat fosforylatie op specifieke plaatsen van het KD van SOBIR1 essentieel is voor het aanzetten van de immuun signalering wanneer RLPs IPs herkennen.

Stroomafwaarts in de signaleringscascade, beginnend bij RLKs die extracellulaire IPs herkennen, spelen 'receptor-like cytoplasmic kinases' (RLCKs) een belangrijke rol. 'Botrytis-Induced Kinase 1' (BIK1) is een belangrijk voorbeeld van een RLCK betrokken bij afweerreacties tegen ziekteverwekkers. BIK1 signaleert stroomafwaarts van welbekende RLKs zoals 'Flagellin-Sensing 2' (FLS2) in Arabidopsis. In 
Hoofdstuk 5 wordt onderzocht of BIK1 ook een rol speelt in de immuun signalering stroomafwaarts van RLP/SOBIR1 complexen. De beschreven experimenten laten zien dat AtBIK1 interacteert met SOBIR1 van Arabidopsis en van tomaat. Met behulp van fylogenetisch onderzoek naar BIK1 homologen in de nachtschadefamilie zijn de BIK1 orthologen van tomaat en $N$. benthamiana geïdentificeerd. Overexpressie van deze BIK1 orthologen bleek de Cf-4/Avr4-geïnduceerde HR te bevorderen. Verrassend genoeg bleek overexpressie van AtBIK1 de Cf-4/Avr4-geïnduceerde HR juist te onderdrukken. Een experiment waarbij de expressie van verscheidene $B I K 1$ homologen in $N$. benthamiana:Cf-4 planten één voor één werd onderdrukt, leidde niet tot de identificatie van NbRLCKs betrokken bij de door Cf-4/Avr4 geïnduceerde HR. Een split-luciferase experiment, waarbij een te onderzoeken eiwit gefuseerd is met de N-terminale helft van het luciferase eiwit, en een mogelijk interacterend eiwit met de C-terminale helft, liet echter zien dat verscheidene RLCKs van tomaat interacteren met SOBIR1 en FLS2. Bovendien bleek één RLCK van tomaat, namelijk 'Tomato Protein Kinase 1b' (SITPK1b), alleen met SOBIR1 te interacteren. Tezamen laten deze resultaten zien dat RLCKs een rol spelen bij de signaal transductie voor het activeren van de verdedigingsreactie door RLP/SOBIR1 complexen.

Het is essentieel dat TM-receptoren strak gereguleerd worden, omdat misregulatie ervoor kan zorgen dat immuunreacties ten onrechte aangezet worden. Deze misregulatie kan leiden tot een slechte ontwikkeling van de plant en zelfs tot systemische celdood. De fysieke scheiding van TM-receptoren door middel van negatieve regulatoren is één van de manieren die wordt gebruikt voor deze regulatie. De familie van de 'BAK1-Interacting RLK' (BIR) eiwitten in Arabidopsis is een groep van eiwitten die zorgt voor negatieve regulatie door middel van het binden aan de co-receptor BAK1. Hierdoor wordt BAK1 weggehouden van signaleringscomplexen waardoor deze niet geactiveerd kunnen worden. In Hoofdstuk 6 wordt getoond dat de BIR eiwitten geconserveerd zijn in de nachtschade familie. Experimenten waarbij de genexpressie van BIR1 of BIR2 wordt onderdrukt, of waarbij BIR1 of BIR2 tot overexpressie wordt gebracht, in planten van de nachtschadefamilie, laten zien dat BIR1 waarschijnlijk een rol speelt in de negatieve regulatie van Cf-4/ SOBIR1 verdedigingsreacties. BIR2 doet dit echter waarschijnlijk niet. Tevens toont overexpressie van gelabelde fusie-eiwitten in N. benthamiana, gevolgd door coimmunoprecipitatie, aan dat SIBIR1 en SIBIR2 interacteren met SIBAK1. Deze resultaten leiden tot de conclusie dat SIBIR1 waarschijnlijk betrokken is bij het reguleren van $\mathrm{Cf}-4 / \mathrm{SOBIR} 1$-gemedieerde verdedigingsreacties, maar SIBIR2 niet. Het is dus waarschijnlijk dat BIR1 en BIR2 met verschillende subgroepen van BAK1 eiwitten op de PM interacteren en dat elke subgroep betrokken is bij het reguleren van een verschillende immuun reactie route. 
In Hoofdstuk 7 worden de bevindingen die in dit proefschrift zijn beschreven samengevat en bediscussieerd. Er wordt geconcludeerd dat alle immuun reacties die beginnen aan het celoppervlak in principe soortgelijk zijn. Wanneer TM-receptoren met een extracellulair LRR domein een ligand herkennen, rekruteren zij allemaal BAK1. Vervolgens vindt er transfosforylatie tussen de verschillende TM-receptoren plaats en wordt de stroomafwaartse signaleringsroute geactiveerd. Dit is zelfs soortgelijk voor verschillende geactiveerde RLP/SOBIR1 complexen, die uiteindelijk tot verschillende intensiteiten van verdedigingsreacties leiden. Op basis van deze kennis wordt voorgesteld om verdedigingsmechanismen niet te onderscheiden op intensiteit, zoals tegenwoordig vaak gedaan wordt, maar om een vernieuwde versie van het invasie model te gebruiken. In dit 'spatiele invasie model' wordt onderscheid gemaakt gebaseerd op de plaats van herkenning van invasie patronen (IPs): extracellulaire IPs (ExIPs) en intracellulaire IPs (InIPs). ExIPs worden herkend door TMreceptoren, wat leidt tot 'extracellularly-triggered immunity' (ExTI), en InIPs worden gedetecteerd door cytoplasmatische receptoren (vaak 'nucleotide-binding leucinerich repeat' receptoren, NB-LRRs), wat leidt tot 'intracellularly-triggered immunity (InTI). Het spatiele invasie model maakt het mogelijk om interacties tussen planten en micro-organismen op moleculair niveau duidelijk te beschrijven. 


\section{Acknowledgements}

The completion of this thesis would not have been possible without the help of many people. Here I would like to take the opportunity to thank all of those who were involved.

First of all, I would like to thank my supervisor and co-promotor Matthieu. Thank you for having faith in me, for your endless enthusiasm and ideas, and the countless fruitful discussions we had. It is amazing that your door is always open. Your answers and feedback are very thorough and useful every time, and I really appreciate that. I wish you all the best in the future at phytopathology and with the Solanaceae (SOL) group.

Also thanks to my promotor Bart, for helpful advice, discussions, and feedback. I wish you all the best in the future.

The SOL group directly felt like a warm bath when I started. Especially because of Laurens. Thank you for all your advice and help. I admire your patience and perseverance in guiding and helping colleagues and students. Good luck, and keep up the good work at phytopathology and the SOL group. The SOL group was also a nice environment because of several former members: my scientific big sister Daniëla, Guozhi, Yu, and Ruby. Thanks for helping me to get started. Special thanks to Thomas for facilitating a good start of my $\mathrm{PhD}$, and also for advice later on.

Richard, thank you for being my external supervisor. Discussions with you helped me think and plan more clearly.

Of course I want to thank my paranymphs. Jinbin, you are a wonderful colleague and friend. You helped me when I had peak periods of stress, and when I had demotivated moments. I really appreciate that you are always honest and clear. Next to working together, I very much enjoyed traveling with you. Especially our trip to China, including a visit to your hometown, I will never forget. Thank you, xie xie, 谢谢. Good luck with finishing your PhD, and planning your future career. I am sure you will do great!

Klaas, thank you for being a helpful colleague and friend. You always lift up the mood in the lab or at the coffee table with nice discussions, stories, and jokes. You are a great travel companion. Without you I would not have seen many nice spots in New Mexico, France, and the Netherlands. I wish you all the best in the future.

I want to thank all the students that have been involved in my project: Tieme, Maria, Sergio, Willemijn, and Jim. Tieme, thanks for being a great first student in the start of my PhD. Maria, thank you for your endless enthusiasm. Sergio, thank you for all the fruitful discussions and your eagerness to learn. I am really happy that you are back as a PhD in the SOL group. You truly lift up the mood with your enthusiasm. Willemijn, thank you for setting up the phosphatase project. Jim, you were an excellent 
last student. Thank you for helping me during the final lab period of my PhD. I wish you all the very best in your future careers.

Wen, thank you for continuing some of the projects that I worked on. Good luck with your PhD.

Many thanks to all colleagues at phytopathology for making my $\mathrm{PhD}$ a great experience. Ester, Natalie, Ali en Grardy, bedankt voor jullie behulpzaamheid, vriendelijkheid en het delen van jullie kennis en ervaring. Jan, bedankt voor het delen van expressiedata en voor je advies. Mireille, Francine en Gert, dank jullie wel voor jullie advies, gezelligheid, en fijne discussies. Also many thanks to Michael, for your patience, help, and advice on bioinformatic matters.

Thanks to my 'escape-room-buddies' Malaika, Jinling, Johan, Chara, Fernando, Nani, and Jasper (on a distance). I really appreciate that we could share difficult, frustrating, desperate, and happy moments during the final periods of our PhDs.

Many thanks to Unifarm personnel, especially Bert, Bertus, Henk, Gerrit, and Taede. Bedankt voor al jullie goede zorgen. Zonder goed verzorgde planten was dit werk niet mogelijk geweest.

Sjef, bedankt voor alle analyses die je hebt gedaan. Met je eindeloze geduld en uitleg heb je me erg geholpen.

Silke and Jelle, thanks for the fruitful collaboration and motivating discussions.

Thanks to my sport-buddies Chara, Xiaoqian, Valeria, and Amalia. You really helped me to get my mind out of work and to relax for a moment.

I really enjoyed the coffee brakes and discussions with you, Cris. Thanks for being a friend, and thanks for your help with statistics. Good luck with the last steps of your $\mathrm{PhD}$, and your future career.

Jos, bedankt dat je een luisterend oor was tijdens moeilijke momenten. Heel veel geluk samen met Jolijn. Jos, Herma en Jan bedankt voor alle gezelligheid en ontspanning.

Although on a distance, Pelin, you are a very dear friend to me. Thank you for listening, and for giving me advice in so many ways. I wish you and Johannes (and ...) all the best in the future.

Laura and Wilfried, thanks for being wonderful friends. Thanks for sharing your career experiences. Good luck all the way up in the north.

Aan al mijn scouting vrienden/collega's ;) bedankt voor alle gezelligheid en ontspanning. Fijn dat ik weer welkom ben na afwezigheid tijdens de laatste periode van mijn PhD. Maja, bedankt voor je inspirerende hulp bij de start van het ontwerpen van de lay-out.

Mirjam, Rosalie en Esther, ook al wonen we niet vlakbij elkaar, jullie vriendschap is heel belangrijk voor me. Bedankt voor alle gezelligheid. Esther, bedankt voor het nalezen van mijn Nederlandse samenvatting. 
Gerbrand, dank je wel voor al je goede zorgen. Je hebt me, vooral tijdens drukke periodes, heel erg geholpen. Bedankt voor al het lekkere eten. Door jou ben ik gezond blijven eten toen ik geen tijd had om fatsoenlijk te koken.

Pap en mam, bedankt voor jullie steun, interesse en goede zorgen. Het is altijd weer heerlijk om bij jullie in Vlaardingen te zijn. Jullie ondersteuning is erg belangrijk voor me. Suzanna, ik ben heel blij met jou als grote zus. Samen met Nina eropuit is altijd gezellig. Succes in de toekomst. Ik hoop dat we elkaar vaak blijven zien. 


\section{About the author}

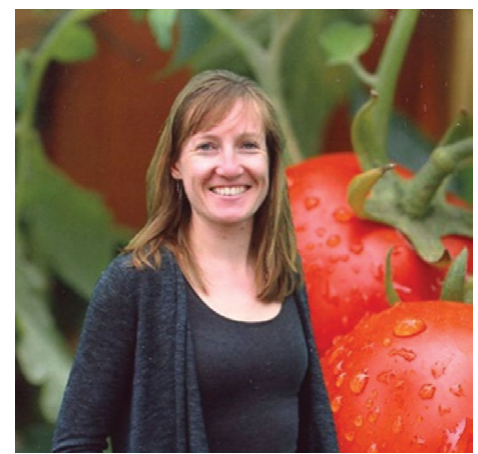

Aranka Margrethe van der Burgh was born on October $6^{\text {th }}, 1986$ in Vlaardingen. After she finished HAVO (2004), she went to Hogeschool Van HallLarenstein in Velp, to study Plant Biotechnology. She conducted her BSc thesis with Dr. Jan Schaart at the Department of Plant Breeding (Wageningen University \& Research), working on the marker-free transformation of strawberry. After completing her BSc, she joined the group of Dr. Henk Schouten at the Department of Plant Breeding as a technician for three years. She worked on the development of cisgenic apples, with the aim to improve fungal resistance and to obtain red fruit flesh.

In 2011, she started the MSc Plant Biotechnology at Wageningen University. During her MSc thesis at the Department of Virology she investigated the possible interaction between a specific viral protein and plant proteins involved in defence against viruses. During the final four months of her MSc, she went to Cologne, Germany, for an internship at the Max Planck Institute for Plant Breeding (MPIPZ). Here, in the Plant Chemetics Group of Dr. Renier van der Hoorn, which recently moved to Oxford, UK, she studied the plant proteasome and its inhibition during bacterial infection.

In 2014, she started her PhD in the Solanaceae (SOL) group (Department of Phytopathology, Wageningen University \& Research) under the supervision of Dr. Matthieu Joosten. The results of the conducted research are described in this thesis. 


\section{List of publications}

van der Burgh AM, Postma J, Robatzek S, Joosten MHAJ. Transphosphorylation between SOBIR1 and BAK1 is required for immune signalling. (submitted).

van der Burgh AM, Huang W, Postma J, Rovenich H, Boeren S, Robatzek S, Joosten MHAJ. Phosphorylation of specific residues of the kinase domain of SOBIR1 is essential for its role in immune signalling. (in preparation).

van der Burgh AM, Joosten MHAJ. Thinking in- and outside the box: extracellularly and intracellularly triggered immunity. (in preparation).

Wu J, van der Burgh AM, Bi G, Zhang L, Alfano JR, Martin G, Joosten MHAJ (2018) The bacterial effector AvrPto targets the regulatory co-receptor SOBIR1 and suppresses defence signalling mediated by the receptor-like protein Cf-4. Molecular Plant-Microbe Interactions 31: 75-85.

Misas-Villamil JC, van der Burgh AM, Grosse-Holz F, Bach-Pages M, Kovács J, Kaschani F, Schilasky S, Emon AEK, Ruben M, Kaiser M, Overkleeft HS, van der Hoorn RAL (2017) Subunit-selective proteasome activity profiling uncovers uncoupled proteasome subunit activities during bacterial infections. The Plant Journal 90: 418-430.

Bi G, Liebrand TWH, Bye RR, Postma J, van der Burgh AM, Robatzek S, Xu X, Joosten MHAJ (2015) SOBIR1 requires the GxxxG dimerization motif in its transmembrane domain to form constitutive complexes with receptor-like proteins. Molecular Plant Pathology 17: 96-107.

Krens FA, Schaart JG, van der Burgh AM, Tinnenbroek-Capel IE, Groenwold R, Kodde LP, Broggini GA, Gessler C, Schouten HJ (2015) Cisgenic apple trees; development, characterization, and performance. Frontiers in Plant Science 6: 286.

Schouten HJ, Brinkhuis J, van der Burgh AM, Schaart JG, Groenwold R, Broggini GA, Gessler C (2014) Cloning and functional characterization of the Rvi15 (Vr2) gene for apple scab resistance. Tree Genetics \& Genomes 10: 251-260.

Soriano JM, Madduri M, Schaart JG, van der Burgh AM, van Kaauwen MP, Tomic L, Groenwold R, Velasco R, van de Weg E, Schouten HJ (2014) Fine mapping of the gene Rvi18 (V25) for broad-spectrum resistance to apple scab, and development of a linked SSR marker suitable for marker-assisted breeding. Molecular Breeding 34: 2021-2032. 


\section{List of abbreviations}

\begin{tabular}{|c|c|}
\hline ACIK1 & Avr9/Cf-9-Induced Kinase 1 \\
\hline Avr & Avirulence \\
\hline BAK1 & BRI1-Associated Kinase 1 (SERK3) \\
\hline BIK1 & Botrytis-Induced Kinase 1 \\
\hline BIR & BAK1-Interacting RLK \\
\hline BKK1 & BAK1-like 1 (SERK4) \\
\hline $\mathrm{BRI1}$ & Brassinosteroid-Insensitive 1 \\
\hline$C f$ & Cladosporium fulvum \\
\hline$C f-4$ & Receptor recognizing Avr4 from C. fulvum \\
\hline CRN & Coryne \\
\hline CLV & Clavata \\
\hline DAMP & Damage-associated molecular pattern \\
\hline ECD & Extracellular domain \\
\hline ECP & Extracellular protein \\
\hline EFR & Elongation Factor-Tu Receptor \\
\hline eGFP & Enhanced Green Fluorescent Protein \\
\hline ETI & Effector-triggered immunity \\
\hline ETS & Effector-triggered susceptibility \\
\hline EVR & Evershed (see SOBIR1) \\
\hline ExIP & Extracellular invasion pattern \\
\hline ExTI & Extracellularly-triggered immunity \\
\hline CDPK & $\mathrm{Ca}^{2+}$-dependent protein kinase \\
\hline CERK1 & Chitin Elicitor Receptor Kinase 1 \\
\hline CST & Cast Away \\
\hline FLS2 & Flagellin-Sensing 2 \\
\hline HAE & Haesa (greek for 'to adhere to') \\
\hline HSL2 & Haesa-like 2 \\
\hline$H R$ & Hypersensitive response \\
\hline IB & Immunoblot \\
\hline IDA & Inflorescence Deficient in Abscission \\
\hline IP & Invasion pattern (see ExIP, InIP) \\
\hline$(\mathrm{CO}-) \mathrm{IP}$ & (co-)immunoprecipitation \\
\hline InIP & Intracellular invasion pattern \\
\hline InTI & Intracellularly-triggered immunity \\
\hline IPRs & IP receptors \\
\hline IPTRs & IP-triggered responses \\
\hline LecRK & Lectin receptor kinase \\
\hline LRR & Leucine-rich repeat \\
\hline
\end{tabular}




$\begin{array}{ll}\text { LysM } & \text { Lysin motif } \\ \text { MAMP } & \text { Microbe-associated molecular pattern } \\ \text { MAPK } & \text { Mitogen-activated protein kinase } \\ \text { MS } & \text { Mass spectrometry } \\ \text { MTI } & \text { MAMP-triggered immunity } \\ \text { MTS } & \text { MAMP-triggered susceptibility } \\ \text { NB-LRR } & \text { Nucleotide-binding leucine-rich repeat } \\ \text { Nev } & \text { Nevershed } \\ \text { NLP } & \text { Necrosis and Ethylene-inducing Peptide 1 (NEP1)-Like Protein } \\ \text { PAMP } & \text { Pathogen-associated molecular pattern } \\ \text { PM } & \text { Plasma membrane } \\ \text { PP } & \text { Protein Phosphatase } \\ \text { PRR } & \text { Pattern recognition receptor } \\ \text { PTI } & \text { PAMP-triggered immunity (see MTI) } \\ \text { RBOHD } & \text { Respiratory Burst Oxidase Homologue D } \\ \text { RCR3 } & \text { Required for Cladosporium Resistance 3 } \\ \text { RLCK } & \text { Receptor-like cytoplasmic kinase } \\ \text { RLK } & \text { Receptor-like kinase } \\ \text { RLP } & \text { Receptor-like protein } \\ \text { ROS } & \text { Reactive oxygen species } \\ \text { R protein } & \text { Resistance protein } \\ \text { SERK } & \text { Somatic Embryogenesis Receptor Kinase } \\ \text { SOBIR1 } & \text { Suppressor Of BIR1 (see EVR) } \\ \text { TM } & \text { Transmembrane } \\ \text { TMM } & \text { Too Many Mouths } \\ \text { TRV } & \text { Tobacco rattle virus } \\ \text { VIGS } & \text { Virus-induced gene silencing } \\ \text { Vir } & \text { Virulence } \\ \text { VT } & \text { Virulence target } \\ & \end{array}$




\title{
Education Statement of the Graduate School Experimental Plant Sciences
}

\author{
Issued to: Aranka M. van der Burgh \\ Date: 21 September 2018 \\ Group: Laboratory of Phytopathology \\ University: Wageningen University \& Research
}

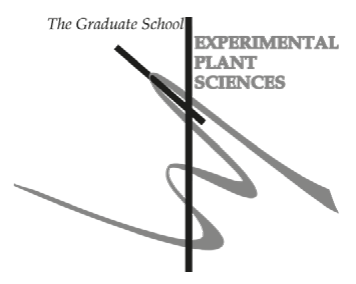

1) Start-up phase

date

- First presentation of your project

Progress of research on receptor-like kinase SOBIR1

21 Nov 212015

- Writing or rewriting a project proposal

- Writing a review or book chapter

- MSc courses

- Laboratory use of isotopes

2) Scientific Exposure

Subtotal Start-up Phase

1.5 credits*

- EPS PhD student days

EPS PhD student day 'Get2Gether, Soest, NL

29-30 Jan, 2015

EPS PhD student day 'Get2Gether, Soest, NL

28-29 Jan 2016

European Retreat for PhD Students in Experimental Plant Sciences, Barcelona, Spain

20-23 Jun 2016

EPS PhD student day 'Get2Gether, Soest, NL

09-10 Feb 2017

- EPS theme symposia

EPS theme 2 symposium 'Interactions between Plants and Biotic Agents', together with Willie Commelin Scholten Day, Amsterdam, NL

25 Feb 2014

EPS theme 2 symposium 'Interactions between Plants and Biotic Agents', together with Willie Commelin Scholten Day, Leiden, NL

22 Jan 2016

EPS theme 2 symposium 'Interactions between Plants and Biotic Agents', together with Willie Commelin Scholten Day, Wageningen, NL

23 Jan 2017

EPS theme 2 symposium 'Interactions between Plants and Biotic Agents', together with Willie Commelin Scholten Day, Amsterdam, NL

$24 \operatorname{Jan} 2018$

- National meetings (e.g. Lunteren days) and other National Platforms

Annual Meeting 'Experimental Plant Sciences' Lunteren, NL

14-15 Apr 2014

Annual Meeting 'Experimental Plant Sciences' Lunteren, NL

13-14 Apr 2015

Annual Meeting 'Experimental Plant Sciences' Lunteren, NL

11-12 Apr 2016

Annual Meeting 'Experimental Plant Sciences' Lunteren, NL

10-11 Apr 2017

- Seminars (series), workshops and symposia

Invited Seminar Dr. Olga del Pozo, Spain

20 Feb 2014

PSG Seminar Dr. Geurts and Prof Dr Kiers

11 Mar 2014

Invited Seminar Dr. Jeroen Mesters

31 Mar 2014 
Invited Seminars Dr. Jane Parker and Dr. Frank van Breusegem

09 Apr 2014

TransPlant workshop, Wageningen, NL

13-14 Oct 2014

Invited Seminar Dr. Michael Freitag

21 Oct 2014

EPS symposium Omics Advances for Academia and Industry - Towards True Molecular Plant Breeding, Wageningen, NL

11 Dec 2014

Invited Seminar Prof. Dr. Yves van de Peer

03 Feb 2015

Invited seminar Dr. Jane Parker

21 Jan 2016

Gender bias awareness, Esther Mollema

26 Jan 2016

Wees seminar Dr. Dan Tawfik

16 Jun 2016

Public lecture 'Rewriting our genes?' Edze Westra and Jennifer Doudna

30 Sep 2016

Invited seminar Dr. Birgit Kemmerling

25 Nov 2016

WURomics symposium

15 Dec 2016

Invited seminar Dr. Richard Kormelink

09 Jun 2017

Seminar Professor Andrew Allan

29 Jun 2017

Seminar Biorhythms, Concertgebouw Amsterdam

04 Oct 2017

Invited seminar Prof. Dr. Giles Oldroyd

19 Oct 2017

Invited seminar Dr. Marjon de Vos

20 Oct 2017

Symposium Future Biochemistry, Wageningen, NL

26 Oct 2017

NVPW (Netherlands Society for Plant Biotechnology and Tissue Culture)

autumn symposium

08 Dec 2017

\section{- Seminar plus}

\section{- International symposia and congresses}

International congress on molecular plant-microbe interactions (MPMI), Rhodos, Greece

Keystone meeting B3: plant receptor kinases, Taos, New Mexico, USA

New phytologist workshop "The apoplast as battleground for plant-microbe interactions", Castle Rauischholzhausen, Germany

Annual conference COST (FA1208) Pathogen-informed strategies for sustainable broad-spectrum crop resistance, Banyuls sur Mer, France Annual conference COST (FA1208) Pathogen-informed strategies for sustainable broad-spectrum crop resistance, Bled, Slovenia International Conference on Biotic Plant Interactions (ICBPI), Xiamen, China

06-10 Jul 2014

08-13 Feb 2015

09-10 Jul 2015

17-19 Feb 2016

01-03 Mar 2017

18-21 Aug 2017

- Presentations

Poster: MPMI meeting, Rhodos, Greece

06-10 Jul 2014

Talk: Keystone meeting, Taos, New Mexico, USA

08-13 Feb 2015

Talk and Poster: Summerschool, Utrecht, NL

24-26 Aug 2015

Talk: EPS theme 2 symposium, Leiden, NL

22 Jan 2016

Poster: Annual conference COST (FA1208), Banyuls sur Mer, France

18 Feb 2016

Talk: EPSR, Barcelona, Spain

22 Jun 2016

Talk: Annual conference COST (FA1208), Bled, Slovenia

02 Mar 2017

Poster: ICBPI conference, Xiamen, China

19 Aug 2017

Talk: EPS Theme 2 symposium, Amsterdam, NL

24 Jan 2018 


\section{- IAB interview}

\section{- Excursions}

Company visit In2care and Genetwister

19 Sep 2014

Subtotal Scientific Exposure 27.0 credits*

3) In-Depth Studies date

- EPS courses or other PhD courses

EPS Spring School 'Host-Microbe Interactomics', Wageningen, NL

02-06 Jun 2014

Advanced course 'Bioinformatics - A User's Approach', Wageningen, NL

25-29 Aug 2014

Advanced course 'Proteomics', Wageningen, NL

27 Apr-01 May 2015

Utrecht Summerschool: Environmental signaling in plants, Utrecht, NL

24-26 Aug 2015

Data analysis and visualizations in R (for biologists), Wageningen, NL

12-13 Dec 2016

- Journal club

Member of a literature discussion group at Phytopathology

2014-2018

- Individual research training

4) Personal development

Subtotal In-Depth Studies

8.3 credits*

- Skill training courses

PhD competence assessments, Wageningen, NL

25 Mar 2014

ExPectationS day (EPS Career day), Wageningen, NL

28 Mar 2014

Interpersonal communication for PhD students, Wageningen, NL

10-11 Apr 2014

PhD carousel, Wageningen, NL

17 Apr 2015

Insight Out: the conference for women in science, Wageningen, NL

24 May 2016

Scientific writing, Wageningen, NL

May-Jul 2017

Last stretch of the PhD, Wageningen, NL

22 Sep 2017

Career orientation, Wageningen, NL

$03,10,17,24,31$

Oct 2017

- Organisation of PhD students day, course or conference

- Membership of Board, Committee or PhD council

Chair of weekly SOL-group meetings

$2015-2018$

Subtotal Personal Development

6.1 credits* $^{*}$

TOTAL NUMBER OF CREDIT POINTS*

42.9

Herewith the Graduate School declares that the PhD candidate has complied with the educational requirements set by the Educational Committee of EPS which comprises of a minimum total of 30 ECTS credits

${ }^{*} A$ credit represents a normative study load of 28 hours of study. 
This research was conducted at the Laboratory of Phytopathology, Wageningen University \& Research, The Netherlands. The work was financially supported by a research grant from the Netherlands Organization for Scientific Research (NWO), division Earth and Life Sciences (ALW).

Cover and layout design by lliana Boshoven Gkini | AgileColor.com Printed by GVO drukkers \& vormgevers B.V. | gvo.nl 
